\title{
A Unitary Perturbation Theory Approach to Real-Time Evolution in the Hubbard Model
}

Dissertation

zur Erlangung des mathematisch-naturwissenschaftlichen Doktorgrades

„Doctor rerum naturalium“

der Georg-August-Universität Göttingen

im Promotionsprogramm ProPhys

der Georg-August University School of Science (GAUSS)

vorgelegt von

Manuel Kreye

aus Georgsmarienhütte

Göttingen, 2019 


\section{Betreuungsausschuss:}

Prof. Dr. Stefan Kehrein

Institut für Theoretische Physik

Georg-August-Universität Göttingen

Prof. Dr. Laura Covi

Institut für Theoretische Physik

Georg-August-Universität Göttingen

\section{Mitglieder der Prüfungskommission:}

Referent:

Prof. Dr. Stefan Kehrein

Institut für Theoretische Physik

Georg-August-Universität Göttingen

Korreferentin:

Prof. Dr. Laura Covi

Institut für Theoretische Physik

Georg-August-Universität Göttingen

Weitere Mitglieder der Prüfungskommission:

Prof. Dr. Fabian Heidrich-Meisner

Institut für Theoretische Physik

Georg-August-Universität Göttingen

Prof. Dr. Peter E. Blöchl

Institut für Theoretische Physik

Technische Universität Clausthal

Prof. Dr. Stefan Mathias

I. Physikalisches Institut

Georg-August-Universität Göttingen

PD Dr. Martin Wenderoth

IV. Physikalisches Institut

Georg-August-Universität Göttingen

Tag der mündlichen Prüfung: 23. Oktober 2019 


\begin{abstract}
We advance perturbative approaches for out-of-equilibrium quantum many-body systems by applying unitary perturbation theory to the real-time evolution of the weakly interacting HUBBARD model in $d>1$ dimensions. We derive general expressions for the fermionic operators that allow us to perturbatively calculate the time evolution of observables to second-order expansion in the interaction strength. We find that the results are stable up to and including the prethermalization regime. We apply our findings to the nonequilibrium build-up of density-density correlations after a weak interaction quench and compare the prethermalization values to equilibrium correlations. Furthermore, we analyze the light-cone-like structure of the density-density susceptibility and the equal-time correlation function in the context of the LIEB-ROBINSON bounds.
\end{abstract}




\section{Contents}

\begin{tabular}{lll}
\hline Introduction & 9
\end{tabular}

1 Motivation 11

1.1 Experiments in far-from-equilibrium situations . . . . . . . . . . . 11

1.1.1 Ultrafast optical spectroscopy $\ldots \ldots \ldots \ldots \ldots \ldots$

1.1.2 Quantum simulations with cold atoms . . . . . . . . . . . 13

1.2 Theoretical considerations $\ldots \ldots \ldots \ldots \ldots \ldots \ldots$

1.2 .1 Thermalization . . . . . . . . . . . . . . . . . 15

1.2 .2 Build-up of correlations $\ldots \ldots \ldots \ldots \ldots \ldots \ldots$

1.2 .3 Scarceness of computational methods . . . . . . . . . . . . 16

1.3 Scope of this thesis $\ldots \ldots \ldots \ldots \ldots \ldots \ldots \ldots$

II Methods, Models and Phenomena 21

2 Unitary perturbation theory 23

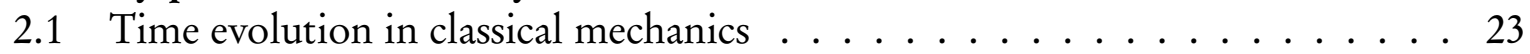

2.1 .1 Naive perturbative expansion . . . . . . . . . . . . . . . 24

2.1 .2 Canonical perturbation theory $\ldots \ldots \ldots \ldots \ldots \ldots \ldots$

2.2 Time evolution in quantum mechanics $\ldots \ldots \ldots \ldots \ldots \ldots$

2.2 .1 Unitary perturbation theory] . . . . . . . . . . . . . . . . 28

2.3 The flow equation method $\ldots \ldots \ldots \ldots \ldots \ldots \ldots$

2.3 .1 Transformation of observables . . . . . . . . . . . . . . . 31

3 Interacting electrons on a lattice 33

3.1 The HubBard model . . . . . . . . . . . . . . . . . . . . . . 34

3.2 Limit of infinite spatial dimensions $\ldots \ldots \ldots \ldots \ldots \ldots$

3.2 .1 Rescaling of the HubBard model . . . . . . . . . . . 36

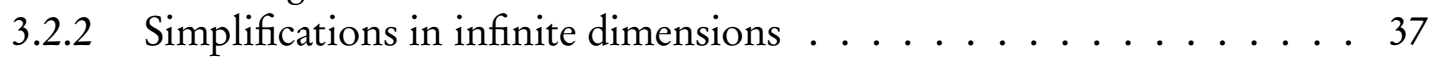

$3.2 .3 \quad$ Physical justification of the limit . . . . . . . . . . . . . . . 37

3.3 Experiments with cold atoms $\ldots \ldots \ldots \ldots \ldots \ldots$

$3.3 .1 \quad$ Experimental setup . . . . . . . . . . . . . . . . . . . 38

3.3 .2 Spatial correlation functions . . . . . . . . . . . . . . . . . . . . 39

3.3.3 Experiments with fermions . . . . . . . . . . . . . . . . 40

3.4 Time-resolved ARPES . . . . . . . . . . . . . . . . . . . . . . 41

$3.4 .1 \quad$ Angle-resolved photoemission spectroscopy . . . . . . . . . . . . 41

3.4 .2 Pump-probe spectroscopy $\ldots \ldots \ldots \ldots \ldots \ldots$. . . . . . . 42 
4 Nonequilibrium phenomena 45

4.1 Prethermalization . . . . . . . . . . . . . . . . . . 45

4.1 .1 Time scale separation. . . . . . . . . . . . . . . . 45

4.1 .2 Generalized Gibbs ensemble . . . . . . . . . . . . . . . . . . 47

4.2 LIEB-RoBINSON bounds . . . . . . . . . . . . . . . . . . . . . . . . 49

4.2 .1 Connection to linear response theory . . . . . . . . . . . 49

4.2 .2 Equal-time connected correlation functions . . . . . . . . . . . 50

III Calculations and Results 53

5 Flow equation analysis of the HuBbard model $\quad 55$

5.1 Quantum quench protocol $\ldots \ldots \ldots \ldots \ldots \ldots \ldots$

5.2 Canonical generator $\ldots \ldots \ldots \ldots \ldots \ldots \ldots \ldots$

5.3 Transformation of the Hamiltonian $\ldots \ldots \ldots \ldots \ldots \ldots$

5.3 .1 First-order expansion $\ldots \ldots \ldots \ldots \ldots \ldots \ldots \ldots$

5.3 .2 Second-order expansion . . . . . . . . . . . . . . . . . . 59

5.3 .3 Energy-diagonal Hamiltonian . . . . . . . . . . . . . . . 62

5.4 Transformation of the annihilation operator $\ldots \ldots \ldots \ldots$

5.5 Time evolution of the annihilation operator $\ldots \ldots \ldots \ldots \ldots$

5.5 .1 Backward transformation $\ldots \ldots \ldots \ldots$. . . . . . 68

5.6 Time scale analysis $\ldots \ldots \ldots \ldots \ldots \ldots \ldots \ldots$

\begin{tabular}{lll}
\hline & Build-up of density-density correlations & 73
\end{tabular}

6.1 General structure of the one-particle density operator $\ldots \ldots \ldots \ldots 75$

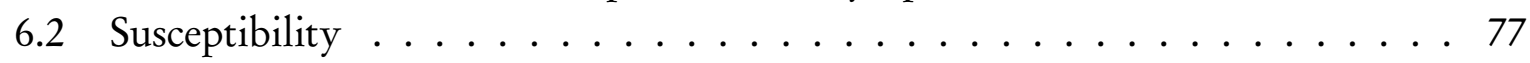

6.2 .1 For antiparallel spins $\ldots \ldots \ldots \ldots \ldots \ldots \ldots$

6.2 .2 For parallel spins $\ldots \ldots \ldots \ldots \ldots \ldots \ldots \ldots \ldots$

6.3 Equal-time correlation function $\ldots \ldots \ldots \ldots \ldots \ldots \ldots \ldots$

6.3 .1 For antiparallel spins $\ldots \ldots \ldots \ldots \ldots \ldots \ldots$

6.3 .2 For parallel spins . . . . . . . . . . . . . . . . . 83

6.4 Correlation function in equilibrium $\ldots \ldots \ldots \ldots \ldots$

$6.4 .1 \quad$ For antiparallel spins $\ldots \ldots \ldots \ldots$. . . . . . . . . . . . 87

6.4 .2 For parallel spins . . . . . . . . . . . . . . . . . . . . . . 89

6.5 Correlation function in infinite dimensions $\ldots \ldots \ldots$. . . . . . 90

6.5 .1 FoURIER transformation to momentum space. . . . . . . . . . . . 91

6.5 .2 Energy integrals in infinite spatial dimensions $\ldots \ldots \ldots \ldots . . . .99$

6.5 .3 Expansion for small momentum . . . . . . . . . . . . 97

\begin{tabular}{lll}
\hline & Spatial decay of density-density correlations & 101
\end{tabular}

7.1 Stationary phase approximation $\ldots \ldots \ldots \ldots 10 \ldots$

$7.1 .1 \quad$ Susceptibilities . . . . . . . . . . . . . . . . . . . 103

7.1 .2 Correlation function . . . . . . . . . . . . . . . . . . . 103

7.2 Algebraically decaying functions in FouRIER space $\ldots \ldots \ldots \ldots \ldots$ 
\begin{tabular}{ll}
\hline IV Discussion & 107
\end{tabular}

8 Conclusions and outlook 109

8.1 Reliability of the method . . . . . . . . . . . . . . . . . . . . 109

8.2 Calculation of density-density correlations . . . . . . . . . . . . . . . . . . . . . . . . . . . .

8.3 Prethermalization of correlation functions . . . . . . . . . . . . . 110

8.4 LIEB-Robinson bounds . . . . . . . . . . . . . . . . . . . . . . . 111

8.5 Outlook . . . . . . . . . . . . . . . . . . . . . . 112

\begin{tabular}{ll}
\hline Acknowledgements & 115
\end{tabular}

$\begin{array}{ll}\text { Appendix } & 119\end{array}$

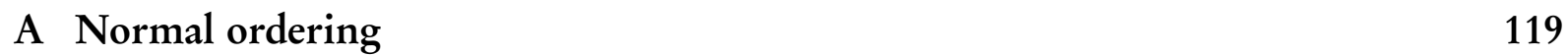

A.1 Definition for fermionic operators . . . . . . . . . . . . . . . . . . 119

A.2 Normal-ordered expressions . . . . . . . . . . . . . . . . . . . . . . 120

A.3 Products of normal-ordered expressions . . . . . . . . . . . . . . . . 120

A.4 Expectation values of normal-ordered expressions . . . . . . . . . . . . 121

\begin{tabular}{ll}
\hline B Consistency checks & 123
\end{tabular}

B.1 Preservation of the canonical anticommutation relation . . . . . . . . 123

B.2 Total spin-up particle number . . . . . . . . . . . . . . . . . . . 127

B.3 Variance of the total spin-up particle number . . . . . . . . . . . . . 129 


\section{Part I}

\section{Introduction}





\section{Chapter 1}

\section{Motivation}

After the development of quantum mechanics in the 1920s physicists became interested in describing many-body systems, like electrons in a solid, within the framework of the new theory. For many years, the investigation focused on systems in thermal equilibrium where a statistical treatment is possible.

Recently, novel experimental techniques like time- and angle-resolved photoemission spectroscopy (trARPES) [11] and quantum simulations with ultracold atoms [9, 72] have opened up the realm of nonequilibrium physics to exploration. This has generated a huge interest in studying quantum systems far from equilibrium, both experimentally and theoretically.

On the experimental side, the investigation of nonequilibrium systems is driven by the discovery of hidden phases that are not present in the equilibrium phase diagrams [38, 57, 109]. At the same time, dynamical surveys benefit from the excellent control over system parameters that is given in simulations with cold atoms. Newfound phenomena have stimulated theoretical research on nonequilibrium dynamics including the question how nonequilibrium systems reach equilibrium again.

When it comes to correlated electrons, one of the prime models for theoretical investigations is the famous HuBBard model that describes electrons moving on a lattice and subjected to a strictly local interaction [55]. For studying the rich dynamics of this and other quantum many-body models, there is a range of analytical and numerical methods available as well as the possibility to perform quantum simulations with cold atoms. In the last few years, a race between these different approaches has arisen to accurately describe the dynamics of many-body models in states far from equilibrium. In this thesis, we want to advance perturbative approaches to real-time evolution problems, where we focus on the HubBARD model in $d>1$ dimensions and the nonequilibrium build-up of density-density correlations after a weak interaction quench.

\subsection{Experiments in far-from-equilibrium situations}

In the 1980s, the generation of increasingly shorter laser pulses, down to the order of femtoseconds, paved the way for ultrafast optical spectroscopy. This gave experimentalists access to a realm where the system can no longer be treated as being in thermal equilibrium [45]. This was followed by huge efforts to study correlated electrons out of equilibrium. 


\subsubsection{Ultrafast optical spectroscopy}

If one wants to study the behavior of correlated materials far from equilibrium, then a common approach is to perform pump-probe experiments. In this setup, a first laser pulse is sent onto a sample and pumps energy into the system e.g. by exciting electrons or phonons. This induces nonequilibrium dynamics in the sample and a second probe pulse is used after a certain delay time in order to perform a spectroscopic measurement of the sample and its features far from equilibrium. As the electronic dynamics occurs on the time scale of femtoseconds, one needs correspondingly short laser pulses for time-resolved measurements.

A number of nonequilibrium phenomena in excited solids have been observed since the 1990s, for example insulator-metal transitions in manganites after excitation of phonons with x-rays [62, 82, 101] and in $\mathrm{VO}_{2}$ due to photoexcitation of electrons [21].

The optical driving of phonons affects the electrons via changes of the band structure. This can lead to astonishing phenomena such as ultrafast changes of the magnetic order in manganites [41] or the induction of spin precession in rare-earth orthoferrites, which thereby simulates an external magnetic field [91]. Recently, Manкоwsкy et al. demonstrated the ultrafast reversal of the ferroelectric polarization in $\mathrm{LiNbO}_{3}$ by phonon driving [77], which is highly relevant for technical applications.

Photoinduced changes of the lattice order can also generate novel nonequilibrium states which cannot be found in the equilibrium phase diagram. For example, in 2011, IcHIKAWA et al. discovered an insulating phase in $\mathrm{Nd}_{0.5} \mathrm{Sr}_{0.5} \mathrm{MnO}_{3}$, where an electronic gap emerged after the material had been driven out of equilibirum [57]. Another stable hidden state was found by STOJCHEvska et al. in $1 T-\mathrm{TaS}_{2}$ after a single laser excitation. They measured a massive decrease of the electrical resistance, which was only restored after subsequent heating and thereby bringing the material back to its thermodynamical ground state [109].

One of the most interesting phenomena that occurs in driven systems far from equilibrium is the emergence of light-induced superconductivity [20].

In 2011, FAUSTI et al. examined the cuprate $\mathrm{La}_{1.675} \mathrm{Eu}_{0.2} \mathrm{Sr}_{0.125} \mathrm{CuO}_{4}$, which is nonsuperconducting in equilibrium [38]. They photoexcited the material with mid-infrared radiation that is resonant with the phonons associated with the $\mathrm{Cu}-\mathrm{O}$ layers of the sample. A subsequent measurement of the time-dependent reflectivity with a near-infrared probe pulse revealed a sudden change of the reflectivity after $\sim 1 \mathrm{ps,} \mathrm{which} \mathrm{remained} \mathrm{constant} \mathrm{up} \mathrm{to} \mathrm{the} \mathrm{longest}$ measurement time of $\sim 100$ ps. Intriguingly, they did not observe any reflectivity change for photoexcitations when the polarization was orthogonal to the $\mathrm{Cu}-\mathrm{O}$ layers.

The connection between optical properties - such as the reflectivity - and superconducting behavior [4] motivated further investigation of the sample.

In a next step, FAUsti et al. measured the electric field reflectance 5 ps after an excitation for different frequencies of the probe pulse (see Fig. 1.1. C). They observed a characteristic plasma edge near $60 \mathrm{~cm}^{-1}$, a so-called JOSEPHSON plasma resonance (JPR). This resonance, which hints at coherent transport, is a general feature of superconducting cuprates [110].

To underpin their claim that $\mathrm{La}_{1.675} \mathrm{Eu}_{0.2} \mathrm{Sr}_{0.125} \mathrm{CuO}_{4}$ exhibits light-induced superconductivity, FAUSTI et al. measured equilibrium spectra of optimally doped $\mathrm{La}_{1.84} \mathrm{Sr}_{0.16} \mathrm{CuO}_{4}$, a material well-known for its superconducting phase, above and below its criticial temperature $T_{\mathrm{c}}=38 \mathrm{~K}$ (see Fig. 1.1, A). In the nonsuperconducting phase, above $T_{\mathrm{c}}$, this material has a featureless spectrum just like $\mathrm{La}_{1.675} \mathrm{Eu}_{0.2} \mathrm{Sr}_{0.125} \mathrm{CuO}_{4}$ in equilibrium (see Fig. 1.1, B). In con- 

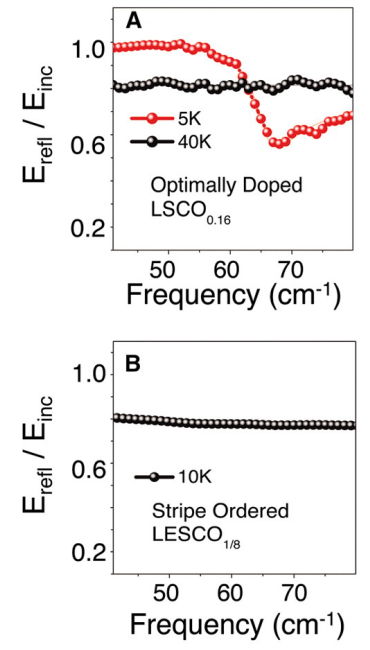

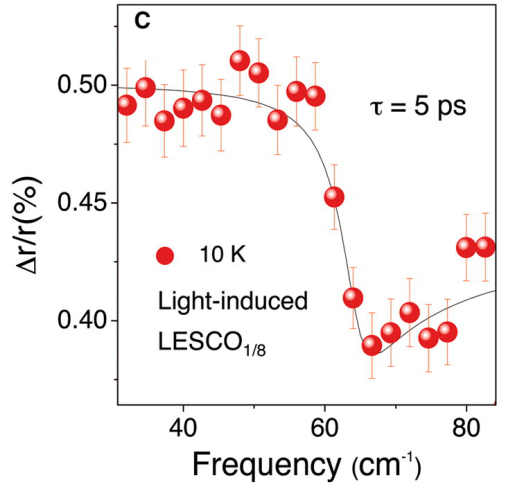

Figure 1.1: Light-induced superconductivity in a cuprate. A: Static electric field reflectance $r=E_{\text {refl }} / E_{\text {inc }}$ of $\mathrm{La}_{1.84} \mathrm{Sr}_{0.16} \mathrm{CuO}_{4}$ above and below $T_{\mathrm{c}}=38 \mathrm{~K}$. B: Static reflectance $r$ of $\mathrm{La}_{1.675} \mathrm{Eu}_{0.2} \mathrm{Sr}_{0.125} \mathrm{CuO}_{4}$ at $T=$ $10 \mathrm{~K}$ shows no sign of superconductivity. C: Transient reflectance $\Delta r$ of $\mathrm{La}_{1.675} \mathrm{Eu}_{0.2} \mathrm{Sr}_{0.125} \mathrm{CuO}_{4}$ after excitation with IR pulses $(\lambda=16 \mathrm{~mm})$. Appearance of a JPR hints at superconductivity. [From Science 331 (2011): "Light-Induced Superconductivity in a Stripe-Ordered Cuprate", FAUSTI et al. Reprinted with permission from AAAS.]

trast, the superconducting phase below $T_{\mathrm{c}}$ is also characterized by the appearance of a JPR.

We will come back to an important technique for pump-probe experiments in sec. 3.4, namely time- and angle-resolved photoemission spectroscopy (trARPES), which is the state-of-the-art method for measuring the electronic band structure and momentum distribution.

\subsubsection{Quantum simulations with cold atoms}

For studying the nonequilibrium dynamics of correlated electrons, an alternative route to ultrafast spectroscopy is given by quantum simulations with ultracold atoms [ [8, 72]].

In these kind of experiments the behavior of electrons in a crystalline solid is simulated by cold atoms in an optical lattice. The artificial setup comes close to many-body models like the HUBBARD model and therefore provides a perfect playground for testing these models against theoretical predictions. In this setup one has excellent control on the model parameters, like the lattice configuration or the interaction strength. Further one can track the dynamics of individual atoms. More details on the experimental implementation will be provided in sec. 3.3 .

In a seminal experiment from 2002, GreINER et al. simulated the BosE-HubBard model by confining a Bose-EINSTEIN condensate of rubidium atoms in a three-dimensional optical lattice [48]. Because the atoms were able to tunnel between neighboring lattice sites, the intitial state was in the superfluid phase. Then, GreINER et al. dynamically increased the lattice depth, which prohibited tunneling, and observed a phase transition to a Мотт insulator. This experimentally verified the well-known Мотт transition from theoretical considerations on the HubBard model.

In 2006, KinOSHITA et al. discovered the emergence of a nonthermal steady state in an ultracold gas of rubidium atoms [61]. They prepared the BosE gas in a one-dimensional optical trap, with the initial condition of two atomic clouds with opposite momenta. The subsequent time evolution showed a behavior that can be best described by a quantum version of NewTON's cradle (see Fig. 1.2). The atom clouds oscillated within the one-dimensional trap and collided with each other twice per oscillation cycle. Even after dephasing and $~ 1000$ collisions, the oscillations continued and the system did not reach thermal equilibrium. 
Figure 1.2: Absorption images of the 1D Bose gas. Atoms are trapped in 3000 parallel onedimensional tubes with 110 atoms per tube. At $t=0$, each atom is pulsed into a superposition of $\pm 2 \hbar k$ momentum. After variable release times, the atom clouds are photographed. The left- and right-moving atoms collide two times per cicle (at $t=3 \mathrm{~ms}$ and $t=10 \mathrm{~ms}$ ). [Reprinted by permission from Springer Nature Customer Service Centre $\mathrm{GmbH}$ : Springer Nature, Nature 440 ("A quantum Newton's cradle", KinOSHITA et al.) (c) 2006]

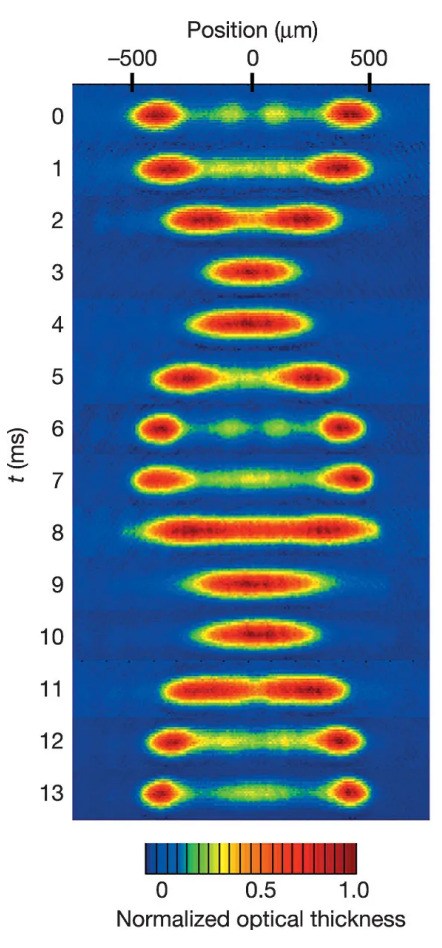

Beyond measurements of particle densities, cold atom experiments also allow for measuring correlation functions [24, 71] . An interesting question in nonequilibrium setups is how correlations between particles build up as a function of space and time.

In an experiment from 2012, CHENEAU et al. examined the parity correlation function in a one-dimensional Bose gas. They prepared the gas in the MOTT insulating phase by releasing it into a deep optical lattice. This was then followed by a quench of the system out of equilibrium by suddenly lowering the lattice depth and a measurement of the parity correlations between different lattice sites as a function of time (see Figs. 3.2 and 3.3). It was found that correlations only build up inside an effective light cone defined by the finite group velocity of quasi-particles.

In the past decades, experiments with cold atoms out of equilibrium have strongly encouraged theoretical research in the equilibration dynamics of quantum many-body systems.

\subsection{Theoretical considerations}

When one analyzes quantum many-body systems, one is confronted with a HILBERT space that grows exponentially in system size. In order to calculate the expectation value of some observable $O$,

$$
\langle O\rangle=\langle\psi|O| \psi\rangle,
$$

for a many-body state $|\psi\rangle$, it is helpful to assume that the system is in thermal equilibrium. This simplifies calculations because the state $|\psi\rangle$ can be replaced by a thermal density matrix

$$
\boldsymbol{\rho}_{\text {th. }}=\frac{1}{Z} e^{-\beta H},
$$


which only depends on the system's Hamiltonian $H$, the inverse temperature $\beta$ and the partition function $Z=\operatorname{Tr}\left[e^{-\beta H}\right]$. In a state described by a thermal density matrix, we can statistically predict expectation values by

$$
\langle O\rangle=\operatorname{Tr}\left[\rho_{\text {th. }} O\right] \text {. }
$$

The assumption of a thermal state breaks down in nonequilibrium settings. Curiously enough, a pure state is described by a density matrix $\rho=|\psi\rangle\langle\psi|$ with $\operatorname{Tr}\left[\boldsymbol{\rho}^{2}\right]=1$ and can therefore never evolve into a mixed thermal state with $\operatorname{Tr}\left[\rho^{2}\right]<1$ under unitary time evolution. This raises the question of how nonequilibrium quantum systems thermalize.

\subsubsection{Thermalization}

In open quantum systems a pure initial state can indeed thermalize because the time evolution is not unitary. The open system is by definition embedded in an environment that it interacts with. While the time evolution of the total system, i.e., open system plus environment, is unitary, this will not be the case if we reduce our view to the embedded system.

The state of the open system is described by a reduced density matrix that is obtained from the total density matrix by tracing out the environment degrees of freedom. The equation of motion for this reduced density matrix usually involves a dissipation term that breaks unitarity, so that a pure state can reach a thermal state depending on the interaction between the open system and its environment [13].

In isolated systems - for example cold atoms in optical lattices are sufficiently isolated from their environment - the above argument does not hold because the time evolution is unitary. However, for certain observables $A$, the expectation value $\langle\psi(t)|A| \psi(t)\rangle$ can become indistinguishable from a thermal prediction $\operatorname{Tr}\left[\rho_{\text {th. }} A\right]$ in the long-time limit. For local observables the interpretation is that the environment effectively acts as a bath, just like in open quantum systems.

In systems that are described by an integrable model the full set of constants of motion constrain the dynamics and prevent observables from thermalizing. For example, the experiment by Kinoshita et al. simulated such an integrable model [61]. However, nonthermal steady states in integrable systems can still be described by a so-called generalized Gibbs ensemble $\boldsymbol{\rho}_{\mathrm{GGE}}$ that depends on the constants of motion [100]. Several such nonthermal steady states were found in analytical calculations [22, 31, 100].

In nonintegrable systems, the interactions in the Hamiltonian lead to sufficient ergodicity for certain observables and hence to thermalization [97].

In the transition region, i.e., in weakly perturbed systems that are close to integrability, the thermalization of some observables can be delayed due to the formation of so-called prethermalization plateaus in the time evolution [7, 84]. In the prethermal regime expectation values can also be predicted by a generalized Gibbs ensemble. This leads to the picture that nonthermal steady states, in integrable systems, are infinitely extended prethermal states [65, 70]. The issue of prethermalization will be treated in more detail in sec. 4.1 .

\subsubsection{Build-up of correlations}

To understand the equilibration process of quantum systems out of equilibrium, an important question is how fast information can propagate through the system. 
Figure 1.3: Scheme for build-up of

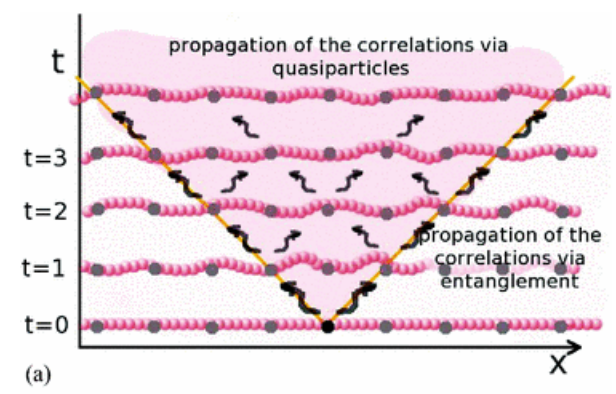
correlations. (a) Long-range entanglement in the initial state. (b) Short-range entanglement in the initial state. Solid circles represent lattice sites, smeared circles represent entanglement between lattice sites. [Reprinted figure with permission from Medvedyeva et al. Physical Review B 88 (2013), 094306 Copyright 2013 by the American Physical Society.]

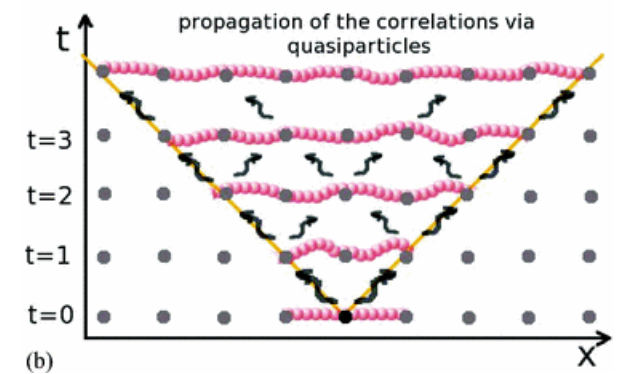

In 1972, LIEB and ROBINSON found a mathematical bound for the propagation of information [74]. The so-called Lieb-Robinson bounds and their generalizations [90] state that in a lattice system with short-range interactions information can only propagate with a finite group velocity $v$. The mathematical statement is that two local observables $\boldsymbol{O}_{X}(t)$ and $\boldsymbol{O}_{Y}(0)$ effectively commute for distances $d(X, Y)>v t$, i.e., outside an effective light cone defined by the group velocity $v$. In other words, the susceptibility $\chi_{X, Y}(t)=-i\left\langle\left[\boldsymbol{O}_{X}(t), \boldsymbol{O}_{Y}(0)\right]_{-}\right\rangle$decays at least exponentially in space outside the light cone.

While the original LIEB-RoBinson bound is a statement on commutators, i.e. susceptibilities, cold atom experiments, like e.g. the one by CHENEAu et al. [24], also consider correlation functions which are related to anticommutators. In fact, these correlation functions are bound inside an effective light cone only if the initial correlations are exponentially suppressed in space. If there are long-range correlations in the initial state, additional correlations can also build up outside the effective light cone [59, 80]. This is illustrated in Fig. 1.3. The correlations inside the light cone propagate via quasi-particles with a maximum group velocity, while correlations outside the light cone emerge due to entanglement.

The spreading of correlations in quantum many-body systems has been extensively studied e.g. with the time-dependent density matrix renormalization group (tDMRG) $[25,28,76,78$, 115], time-dependent variational Monte Carlo [18, 23], artificial neural network techniques [19] and analytical methods [1, 10, 15, 16, 17, 36, 80].

A quantitative treatment of LIEB-RoBINSON bounds for susceptibilities and equal-time correlation functions will be given in sec. 4.2 .

\subsubsection{Scarceness of computational methods}

While much effort has been spent on the theoretical discussion of nonequilibrium problems, there are still severe difficulties in treating the real-time evolution of quantum many-body systems analytically and numerically for sufficiently long times. Exemplarily, we will touch on problems arising in tDMRG, quantum Monte Carlo (QMC) algorithms and perturbative approaches. 
The DMRG method is a variational technique for obtaining the ground state of one-dimensional Hamiltonians. Here, one utilizes the fact that in one-dimensional systems the ground state has a low entanglement entropy given by an area law. This justifies a matrix product state ansatz for the wave function. However, when one wants to analyze the time evolution in tDMRG, one is confronted with the problem that the entanglement entropy grows linearly in time, which makes tDMRG unstable after certain time steps [15].

While the DMRG method works well for one-dimensional systems, in dynamical mean-field theory (DMFT) one explores the opposite limit of infinite spatial dimensions or to be precise of infinite lattice coordination number. This limit, which we will discuss in sec. 3.2, allows one to map the many-body lattice Hamiltonian onto a local impurity problem. These impurity models can be solved numerically, e.g. by QMC algorithms. If one wants to track the time evolution in nonequilibrium setups, one has to estimate integrals of functions $e^{i H t}$ that become rapidly oscillating with growing time. Thereby, QMC suffers from an exponential scaling of computational cost with time known as the dynamical sign problem. With some improvements one can at least achieve quadratic scaling, but this still leads to unstability at intermediate times [27].

In an analytical treatment of a weakly perturbed system we may try to solve the HEISENBERG equation of motion for a certain observable,

$$
\frac{\mathrm{d} O(t)}{\mathrm{d} t}=i[H, O(t)]_{-},
$$

perturbatively. Let us consider, for example, a system described by the Hamiltonian $H=$ $H_{0}+g H_{1}$ with a weak coupling strength $g \ll 1$. In order to approximately solve the equation of motion for $O$, we expand the operator in orders of the coupling strength, i.e.,

$$
\boldsymbol{O}(t)=\boldsymbol{O}^{(0)}(t)+g \mathrm{O}^{(1)}(t)+g^{2} \mathrm{O}^{(2)}(t)+O\left(g^{3}\right) .
$$

Inserting this ansatz into the equation of motion allows for a successive solution of the contributions for different orders in $g$.

A common problem arises from the fact that we have not just expanded $O(t)$ in orders of $g$ but actually in orders of $g$ and $t$. This leads to so-called secular terms in the perturbative solution that grow with some power law in time, e.g. $O^{(n)}(t) \propto t^{m}$. Clearly, these terms become unstable for intermediate times.

HACKL and KEHREIN demonstrated that we can avoid secular terms if we solve the HEISENBERG equations of motion in a basis where the Hamiltonian is approximately energy-diagonal [51]. This is systematically explored in the framework of unitary perturbation theory, which we will discuss in chapter 2 .

\subsection{Scope of this thesis}

Motivated by the experimental advances in pump-probe spectroscopy and quantum simulations with cold atoms, we study the nonequilibrium dynamics of the HuBBARD model after a weak interaction quench. Analyzing the real-time evolution is an important step towards understanding the behavior of correlated electrons far from equilibrium.

In the current race between computational methods, we want to demonstrate the usefulness 
of unitary perturbation theory that gives analytical insights into the real-time evolution of weakly perturbed systems and is stable up to and including prethermalization time scales.

In a previous work [84, 85], MOECKEL and KeHREIN successfully applied unitary perturbation theory to the HuBBaRD model as they calculated the time evolution of the momentum distribution function $n_{k}(t)$ after a weak quench from the noninteracting to the interacting system. Their perturbative analysis allowed for a treatment of expectation values of one-particle functions up to second order in the interaction strength.

We will build on their calculations and extend them to second-order expansions of twoparticle functions. As an important application, we will explicitly calculate density-density susceptibilities and the corresponding equal-time correlation functions. On the one hand, we can study the prethermalization dynamics of these quantities, while on the other hand, these functions provide a non-trivial test of the LIEB-ROBINSON bounds.

We point out that our general perturbative results for the time evolution in the HUBBARD model in $d>1$ dimensions open up the playground for a variety of further investigations because they enable the calculation of a wide class of observables, where the density-density susceptibilities and correlation functions are only two examples.

\section{Outline}

In chapter 2, we will systematically introduce the method of unitary perturbation theory. We will motivate the main idea that is based on the well-known canonical perturbation theory from classical mechanics. The anharmonic oscillator will serve as a role model to demonstrate how both canoncial and unitary perturbation theory avoid secular terms in the time evolution. We will then present the flow equation method, which will become vital if we want to apply unitary perturbation theory to quantum many-body systems.

An important class of quantum many-body systems are interacting electrons on a lattice. We will discuss this in chapter 3 by taking the HubBaRD model as the prime example to study correlation effects. The DMFT limit is highly relevant for correlated materials and we will show that it simplifies calculations in the HUBBARD model. We will also review how nonequilibrium lattice electrons are simulated in experiments with cold atoms and how correlation effects are studied in real materials by means of trARPES. Two characteristic phenomena that occur in quantum systems out of equilibrium and that we will study in this thesis, prethermalization and the light-cone-like spreading of correlations, will be sketched out in chapter 4.

In chapter 5, we will start our actual analysis of the real-time evolution in the HUBBARD model. We will keep things quite general and solve the HeIsENBERG equation of motion for the fermionic annihilation operator up to second order in the interaction strength and for arbitrary dimension $d>1$ using the flow equation method. The result can be used for the construction of a wide class of observables and is stable up to and including the prethermalization regime.

We will use it to calculate the density-density susceptibilities and equal-time correlation functions for antiparallel and parallel spins in leading-order expansions in chapter 6 . The results from the flow equation analysis enable us to calculate these functions both for a nonequilibrium quench setup and for the interacting system in equilibrium. We will also calculate the prethermalization values of the nonequilibrium correlation functions and find that they are 
equal to the equilibrium values.

In chapter 7, we will finally discuss the spatial decay of the susceptibilities and correlation functions and relate it to the Lieb-Roвinson bounds. We will find that the susceptibilities and the antiparallel-spin correlation function decay faster than algebraically outside an effective light cone, while the parallel-spin correlation function at zero temperature decays algebraically outside the light cone because of the entanglement in the initial state.

We will conclude this thesis in chapter 8 with a discussion of the results and an outlook to prospective research.

Part of this thesis that deals with prethermalization of correlation functions is published at [67]. 


\section{Part II}

\section{Methods, Models and Phenomena}





\section{Chapter 2}

\section{Unitary perturbation theory}

In this chapter, we introduce the method of unitary perturbation theory, which is designed for calculating the real-time evolution of observables in non-integrable quantum many-body systems without producing secular terms that would diverge in the long-time limit. It was first proposed by HACKL and KeHREIN in 2008 [51, 52] as the quantum analog of the wellestablished canonical perturbation theory from classical mechanics.

In order to illustrate the main idea of both schemes, we start with a classical system, a perturbed oscillator, in sec. 2.1. Here, we show that the perturbative treatment of the harmonic oscillator with an additional small anharmonic term will lead to unphysical behavior if we apply the perturbative expansions too naivly. The unphysical terms can be avoided by a canonical transformation of the observables before the perturbative expansions. This procedure then results in the scheme of canonical perturbation theory that is treated in many classical textbooks (see e.g. [47]).

In sec. 2.2, we reformulate this idea in the context of quantum mechanical systems, where the main difference lies in replacing the canonical transformations by unitary transformations, hence the name "unitary perturbation theory".

Finding an appropriate unitary transformation turns out to be difficult when going to quantum many-body systems. A systematic approach that tackles the problem of the continuum of energy scales is given by applying a continuum of infinitesimal unitary transformations. This so-called flow equation method, established by WEGNER in 1994 in the context of condensed matter physics [114] and independently by GEAZEK and WILSON in 1993 in the context of high-energy physics [46], is finally presented in sec. 2.3 of this chapter. For a detailed treatment of the flow equation approach we refer to [60].

\subsection{Time evolution in classical mechanics}

In classical mechanics, the state of a physical system is described by some generalized coordinates $q_{i}$ and their conjugate momenta $p_{i}$. The time evolution of an observable $O\left(q_{i}, p_{i}\right)$ that depends on these coordinates is driven by the Hamiltonian function $H\left(q_{i}, p_{i}\right)$ that typically represents the system's energy. The observable $O$ evolves in time according to the differential equation

$$
\frac{\mathrm{d} O}{\mathrm{~d} t}=\{O, H\}_{q_{i}, p_{i}}+\frac{\partial O}{\partial t},
$$


where the second term on the right-hand side vanishes for observables that are not explicitly time-dependent. The PoIsson brackets $\{\cdot, \cdot\}_{q_{i}, p_{i}}$ are defined by

$$
\{f, g\}_{q_{i}, p_{i}} \stackrel{\text { def }}{=} \sum_{i}\left(\frac{\partial f}{\partial q_{i}} \frac{\partial g}{\partial p_{i}}-\frac{\partial f}{\partial p_{i}} \frac{\partial g}{\partial q_{i}}\right) .
$$

As eq. (2.1) is a first-order differential equation, the initial conditions $q_{i}(t=0)$ and $p_{i}(t=0)$ fully determine the system's time evolution (strictly, if the right-hand side of the differential equation is Lipschitz continuous in O, cf. PICARD-LINDELÖF theorem [75]).

\section{Example: anharmonic oscillator}

A simple system to look at is a one-dimensional harmonic oscillator, whose Hamiltonian $H_{0}$ is given by

$$
H_{0}(q, p)=\frac{1}{2} p^{2}+\frac{1}{2} q^{2}
$$

The equations of motion for the coordinates $q$ and $p$ themselves are then

$$
\begin{aligned}
& \dot{q}=\left\{q, H_{0}\right\}_{q, p}=p, \\
& \dot{p}=\left\{p, H_{0}\right\}_{q, p}=-q .
\end{aligned}
$$

For the initial conditions $q(0)=1$ and $p(0)=0$, the solution to this system of differential equations is

$$
\begin{aligned}
& q(t)=\cos (t), \\
& p(t)=-\sin (t) .
\end{aligned}
$$

If we add a small anharmonic term to the Hamiltonian,

$$
\begin{aligned}
H(q, p) & =H_{0}(q, p)+H_{\text {int }}(q, p), \\
H_{\text {int }}(q, p) & =g \frac{1}{4} q^{4},
\end{aligned}
$$

where $g \ll 1$, the perturbed equations of motion,

$$
\begin{aligned}
& \dot{q}=\{q, H\}_{q, p}=p, \\
& \dot{p}=\{p, H\}_{q, p}=-q-g q^{3},
\end{aligned}
$$

can no longer be solved analytically. However, for small $g$, we might consider a perturbative treatment. This was done, for instance, in [52], and we briefly summarize the idea here.

\subsubsection{Naive perturbative expansion}

In order to get a perturbative solution to the equation of motion

$$
\ddot{q}=-q-g q^{3}
$$


we make an ansatz that is an expansion for small $g$,

$$
q(t)=q^{(0)}(t)+g q^{(1)}(t)+O\left(g^{2}\right) .
$$

If we insert this ansatz into the equation of motion (2.12), the zeroth and first order contributions will be

$$
\begin{aligned}
& \ddot{q}^{(0)}(t)=-q^{(0)}(t), \\
& \ddot{q}^{(1)}(t)=-q^{(1)}(t)-\left(q^{(0)}(t)\right)^{3} .
\end{aligned}
$$

With the initial conditions $q(0)=1$ and $\dot{q}(0)=0$, we arrive at the solutions

$$
\begin{aligned}
& q^{(0)}(t)=\cos (t), \\
& q^{(1)}(t)=-\frac{1}{8} \cos (t) \sin ^{2}(t)-\frac{3}{8} t \sin (t) .
\end{aligned}
$$

The last term, $-\frac{3}{8} t \sin (t)$, is a so-called secular term. It shows unphysical behavior since it grows linearly in time, whereas the form of the anharmonic potential suggests a bound trajectory. Therefore, the naive perturbative treatment of the anharmonic oscillator problem cannot capture the long-term behavior of the system.

\subsubsection{Canonical perturbation theory}

We can avoid secular terms like the one in eq. (2.17) if we bring the Hamiltonian to normal form by means of a canonical transformation $(H, q, p) \rightarrow(K, Q, P)$ before calculating the time evolution. This idea is illustrated in Fig. 2.1. The canonical transformation should be

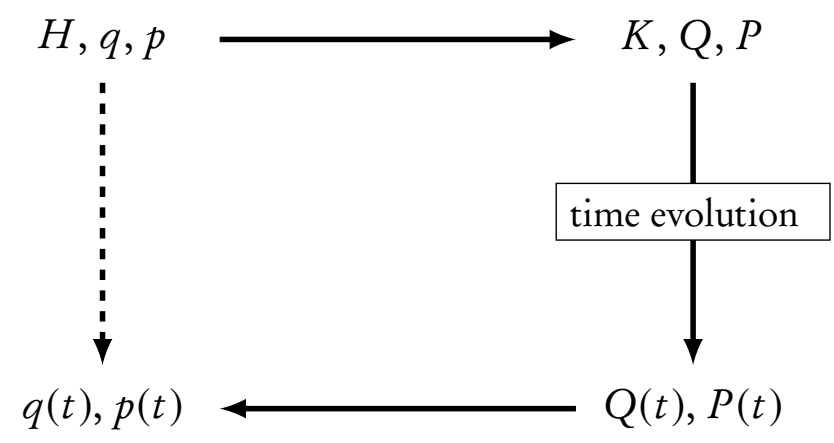

Figure 2.1: Illustration of the canonical perturbation theory scheme

done in a way that the transformed Hamiltonian $K$ does not produce any secular terms in the time evolution of $Q$ and $P$. The absence of secular terms will then still hold after the backward transformation to the original coordinates $q$ and $p$. Interestingly, this will also be the case if we truncate both the canonical transformation and the time evolution in a perturbative manner, meaning after some order of a certain expansion parameter.

For an overview of this so-called "canonical perturbation theory" we refer to classical textbooks, e.g. [47]. 
In our example of the anharmonic oscillator, we choose the corresponding generating function (in accordance with [52]) to be

$$
F_{2}(q, P)=q P+g\left(\frac{5}{32} q^{3} P+\frac{3}{32} q P^{3}\right)
$$

Then, we will get the following transformation rules,

$$
\begin{aligned}
& p=\frac{\partial F_{2}}{\partial q}=P+g\left(\frac{15}{32} q^{2} P+\frac{3}{32} P^{3}\right) \\
& Q=\frac{\partial F_{2}}{\partial P}=q+g\left(\frac{5}{32} q^{3}+\frac{9}{32} q P^{2}\right)
\end{aligned}
$$

As the generating function is not explicitly time-dependent, the Hamiltonian $K=H$ from eq. (2.8) is invariant. We get

$$
\begin{aligned}
H(Q, P) & =\frac{1}{2} p^{2}+\frac{1}{2} q^{2}+g \frac{1}{4} q^{4} \\
& =\frac{1}{2} P^{2}+\frac{1}{2} Q^{2}+g\left(\frac{15}{32} Q^{2} P^{2}+\frac{3}{32} P^{4}-\frac{5}{32} Q^{4}-\frac{9}{32} Q^{2} P^{2}+\frac{1}{4} Q^{4}\right)+O\left(g^{2}\right) \\
& =\frac{1}{2} P^{2}+\frac{1}{2} Q^{2}+g \frac{3}{8}\left(\frac{1}{2} P^{2}+\frac{1}{2} Q^{2}\right)^{2}+O\left(g^{2}\right) \\
& =H_{0}(Q, P)+g \frac{3}{8} H_{0}^{2}(Q, P)+O\left(g^{2}\right) .
\end{aligned}
$$

We recognize that the transformed Hamiltonian depends only on powers of $H_{0}$ (at least in a perturbative expansion up to first order in $g$ ). This so-called "normal-form" simplifies the equations of motion,

$$
\begin{aligned}
\dot{Q} & =\{Q, H\}_{Q, P} \\
& =\left\{Q, H_{0}\right\}_{Q, P}+g \frac{3}{4}\left\{Q, H_{0}\right\}_{Q, P} H_{0}+O\left(g^{2}\right) \\
& =P\left(1+g \frac{3}{4} H_{0}\right)+O\left(g^{2}\right), \\
\dot{P} & =\{P, H\}_{Q, P} \\
& =-Q\left(1+g \frac{3}{4} H_{0}\right)+O\left(g^{2}\right),
\end{aligned}
$$

so that the solution is given by

$$
\begin{aligned}
& Q(t)=Q_{0} \cos \left(\omega_{g} t\right)+O\left(g^{2}\right), \\
& P(t)=-Q_{0} \sin \left(\omega_{g} t\right)+O\left(g^{2}\right),
\end{aligned}
$$




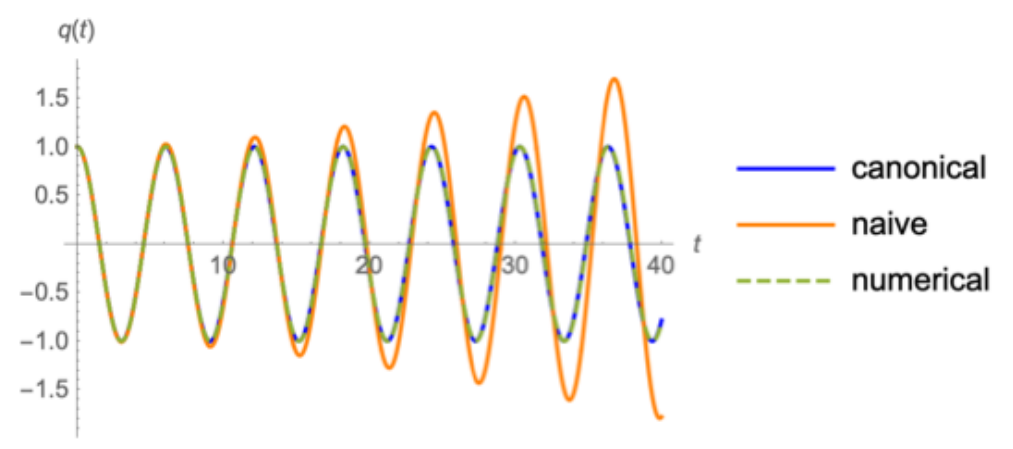

Figure 2.2: Different solutions of the anharmonic oscillator problem (with $g=0.1$ )

with $\omega_{g} \stackrel{\text { def }}{=} 1+g \frac{3}{4} H_{0}$ and $Q_{0} \equiv Q(0)=1+g \frac{5}{32}$.

Finally, transforming back to the original coordinate $q$ yields

$$
\begin{aligned}
q(t) & =Q(t)-g\left(\frac{5}{32} Q^{3}(t)+\frac{9}{32} Q(t) P^{2}(t)\right)+O\left(g^{2}\right) \\
& =\cos \left(\omega_{g} t\right)+g\left(\frac{5}{32} \cos \left(\omega_{g} t\right)-\frac{5}{32} \cos ^{3}\left(\omega_{g} t\right)-\frac{9}{32} \cos \left(\omega_{g} t\right) \sin ^{2}\left(\omega_{g} t\right)\right)+O\left(g^{2}\right) \\
& =\cos \left(\omega_{g} t\right)-g \frac{1}{8} \cos \left(\omega_{g} t\right) \sin ^{2}\left(\omega_{g} t\right)+O\left(g^{2}\right),
\end{aligned}
$$

where we no longer encounter secular terms.

Note that if we expanded the trigonometric functions for small $g$, we would get

$$
q(t) \approx \cos (t)-g \frac{3}{4} H_{0} t \sin (t)-g \frac{1}{8} \cos (t) \sin ^{2}(t)+O\left(g^{2}\right),
$$

which, because $H_{0}=\frac{1}{2}+O(g)$, is just the result from eq. 2.17) that we got from the naive perturbative ansatz.

We conclude that the method of canonical perturbation theory rearranges the terms in the perturbative treatment such that no secular terms appear. To illustrate this, we compare the solutions from naive and canonical perturbation theory to the exact numerical solution in Fig. 2.2. Clearly, the canonical result is much more in line with the numerical one than the naive result.

\section{Relevance of canonical perturbation theory today}

The anharmonic oscillator problem can be thought of as one of the most simple perturbative problems in classical mechanics, because it only deals with a single particle. A more elaborate problem is that of the lunar motion in the earth's orbit, where the gravitational force of the sun plays the role of the small perturbation. In 1867, Delaunay published his perturbative treatment of this three-body problem [30] that contained over 700 pages of calculations with a remaining error that was still $O\left(10^{-4}\right)$. This illustrates the effort one has to take when examining many-body problems with canonical perturbation theory.

Today, in times of effective numerical algorithms for classical many-body problems, canonical perturbation theory has become less important for actual calculations. However, for quantum 
many-body systems, numerical algorithms that can deal with time-evolution are rare and high computing capacities are needed. This is a great starting point to rethink the general scheme again and translate it to the framework of quantum mechanics.

\subsection{Time evolution in quantum mechanics}

Going from classical to quantum systems the observables are represented by self-adjoint operators, e.g. $O\left(q_{i}, p_{i}\right)$, and the PoIsson brackets are replaced by commutators in a way that we have

$$
\begin{aligned}
{\left[\boldsymbol{q}_{i}, \boldsymbol{p}_{j}\right]_{-}=i \delta_{i j}, } \\
{\left[\boldsymbol{q}_{i}, \boldsymbol{q}_{j}\right]_{-}=\left[\boldsymbol{p}_{i}, \boldsymbol{p}_{j}\right]_{-}=0 }
\end{aligned}
$$

as the fundamental commutation relations.

The time evolution of $O$ is driven by the system's Hamiltonian $H$, which is just the Hamiltonian function written as an operator. The corresponding HeISENBERG equation of motion (for a non-explicitly time-dependent observable) is

$$
\frac{\mathrm{d} O}{\mathrm{~d} t}=i[H, O]_{-} .
$$

\section{Example: anharmonic oscillator}

As an example, consider the anharmonic oscillator again. The quantum mechanical Hamiltonian that corresponds to eq. 2.8 is

$$
H=\frac{1}{2} p^{2}+\frac{1}{2} q^{2}+g \frac{1}{4} q^{4},
$$

with the fundamental commutation relation $[q, p]_{-}=i 1$. The latter implies that the HeISENBERG equations of motion are

$$
\begin{aligned}
& \dot{q}=i[H, q]_{-}=p, \\
& \dot{p}=i[H, p]_{-}=-q-g q^{3}
\end{aligned}
$$

These are just the operator versions of the classical equations 2.10) and (2.11). Hence, the same problem with secular terms arises if we try to solve these equations with a naive perturbative ansatz for $q(t)$.

\subsubsection{Unitary perturbation theory}

In order to get a meaningful perturbative solution of the quantum mechanical problem, we apply the same scheme as depicted in Fig. 2.1 for the classical case. However, in the quantum case the transformation must be unitary such that they preserve the canonical commutation relations as well as hermiticity (as proposed in [51, 52]). These not only act on the Hamiltonian and the observables but also on the physical state $|\psi\rangle$ of the system. We label the unitarily transformed quantities with a tilde (cf. Fig. 2.3). 


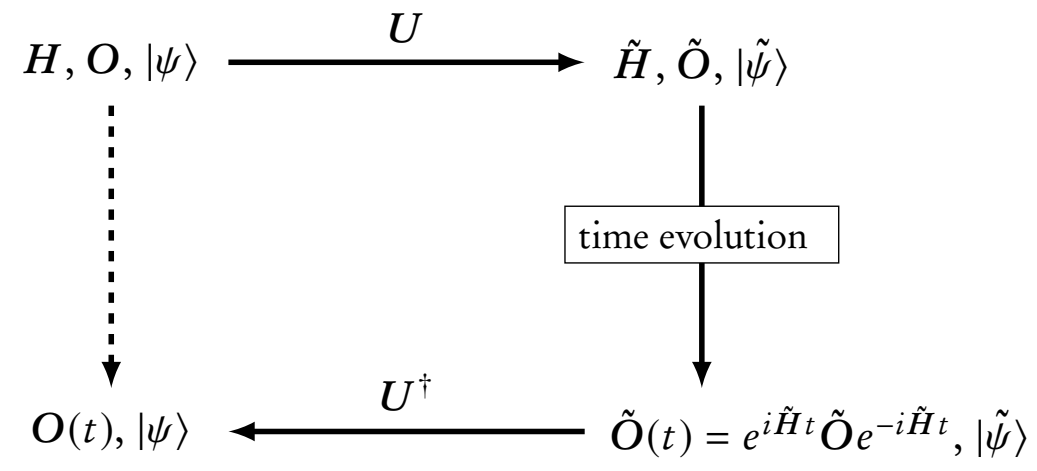

Figure 2.3: Illustration of the unitary perturbation theory scheme

The goal is to find a unitary transformation $U$ that diagonalizes (at least in a perturbative manner) the Hamiltonian such that the time evolution in the new basis is trivial and lacking of secular terms. The difficulty of the scheme is that the observable $O$ must also be transformed under the same unitary transformation, which can lead to a quite complicated structure.

In nonequilibrium problems, we often deal with quantum quenches, where the initial state $|\psi\rangle$ is the ground state of some simple Hamiltonian and the time evolution is given by a more advanced Hamiltonian $\boldsymbol{H}$. Due to the simplicity of $|\psi\rangle$, we do not transform it under $\boldsymbol{U}$. Instead, we apply the backward transformation $U^{\dagger}$ on the time-evolved observable $\tilde{O}(t)$, in order to evaluate expectation values with respect to the original state $|\psi\rangle$.

In equilibrium settings, things are the other way around. Here, the ground state $|\psi\rangle$ of some interacting Hamiltonian $\boldsymbol{H}$ can be quite complicated, while the unitarily transformed state $|\tilde{\psi}\rangle$ is much simpler, because it is just the ground state of the diagonal $\tilde{H}$. Therefore, in equilibrium scenarios, we calculate expectation values in the $B=\infty$-basis and do not need to apply any backward transformation.

\section{Example: anharmonic oscillator}

Considering again the anharmonic oscillator example, our ansatz for the unitary transformation of $q$ and $p$ is motivated by eqs. (2.19) and (2.20):

$$
\begin{aligned}
& \tilde{q}=q+g\left(\frac{5}{32} q^{3}+\frac{9}{32} p q p\right)+O\left(g^{2}\right), \\
& \tilde{p}=p-g\left(\frac{15}{32} q p q+\frac{3}{32} p^{3}\right)+O\left(g^{2}\right) .
\end{aligned}
$$

This is already a perturbative ansatz up to first order in $g$. In this order, the unitarity of the transformation is guaranteed because the fundamental commutation relation

$$
[\tilde{q}, \tilde{p}]_{-}=[q, p]_{-}+O\left(g^{2}\right)
$$

is preserved. With the backward transformation rules

$$
\begin{aligned}
& q=\tilde{q}-g\left(\frac{5}{32} \tilde{q}^{3}+\frac{9}{32} \tilde{p} \tilde{q} \tilde{p}\right)+O\left(g^{2}\right), \\
& p=\tilde{p}+g\left(\frac{15}{32} \tilde{q} \tilde{p} \tilde{q}+\frac{3}{32} \tilde{p}^{3}\right)+O\left(g^{2}\right),
\end{aligned}
$$


we can write down the Hamiltonian

$$
\begin{aligned}
\tilde{H} & =\frac{1}{2} p^{2}+\frac{1}{2} q^{2}+g \frac{1}{4} q^{4} \\
& =\frac{1}{2} \tilde{p}^{2}+\frac{1}{2} \tilde{q}^{2}+g\left(\frac{3}{32} \tilde{p} \tilde{q} \tilde{p} \tilde{q}+\frac{3}{32} \tilde{q} \tilde{p} \tilde{q} \tilde{p}+\frac{3}{32} \tilde{p}^{4}+\frac{3}{32} \tilde{q}^{4}\right)+O\left(g^{2}\right) \\
& =\frac{1}{2} \tilde{p}^{2}+\frac{1}{2} \tilde{q}^{2}+g\left(\frac{3}{32} \tilde{p}^{2} \tilde{q}^{2}+\frac{3}{32} \tilde{q}^{2} \tilde{p}^{2}+\frac{3}{32} \tilde{p}^{4}+\frac{3}{32} \tilde{q}^{4}\right)+g \frac{3}{32}+O\left(g^{2}\right) \\
& =\frac{1}{2} \tilde{p}^{2}+\frac{1}{2} \tilde{q}^{2}+g \frac{3}{8}\left(\frac{1}{2} \tilde{p}^{2}+\frac{1}{2} \tilde{q}^{2}\right)^{2}+g \frac{3}{32}+O\left(g^{2}\right)
\end{aligned}
$$

Hence, the equations of motion in the new basis read

$$
\begin{aligned}
& \dot{\tilde{q}}=i[\tilde{H}, \tilde{q}]_{-}=\tilde{p}+g \frac{3}{8} \tilde{H}_{\circ} \tilde{p}+g \frac{3}{8} \tilde{p} \tilde{H}_{0}+O\left(g^{2}\right), \\
& \dot{\tilde{p}}=i[\tilde{H}, \tilde{p}]_{-}=-\tilde{q}-g \frac{3}{8} \tilde{H} \circ \tilde{q}-g \frac{3}{8} \tilde{q} \tilde{H}_{0}+O\left(g^{2}\right),
\end{aligned}
$$

with $\tilde{H}_{0} \stackrel{\text { def }}{=} \frac{1}{2} \tilde{p}^{2}+\frac{1}{2} \tilde{q}^{2}$ being a constant of motion up to first order in $g$.

To solve these equations of motion, we introduce $|n\rangle$ as the normalized eigenstates of $\tilde{H}_{0}$ with eigenvalues $\tilde{\epsilon}_{n}$. In this basis, the matrix elements of the operators obey

$$
\begin{aligned}
\frac{\mathrm{d}}{\mathrm{d} t}\langle m|\tilde{q}| n\rangle & =\left(1+g \frac{3}{8}\left(\tilde{\epsilon}_{m}+\tilde{\epsilon}_{n}\right)\right)\langle m|\tilde{p}| n\rangle+O\left(g^{2}\right), \\
\frac{\mathrm{d}}{\mathrm{d} t}\langle m|\tilde{p}| n\rangle & =-\left(1+g \frac{3}{8}\left(\tilde{\epsilon}_{m}+\tilde{\epsilon}_{n}\right)\right)\langle m|\tilde{q}| n\rangle+O\left(g^{2}\right) .
\end{aligned}
$$

Similar to the classical case, these differential equations can be integrated without any secular terms appearing.

\subsection{The flow equation method}

In unitary perturbation theory, the essential task lies in finding an appropriate unitary transformation that diagonalizes the Hamiltonian in an approximate way. While we could write down such a transformation for the anharmonic oscillator quite easily, things get more complicated when going to quantum many-body systems. Here, the continuum of energy scales will generate diverging energy denominators for energy eigenvalues that lie close to one another. A way out of this problem is the flow equation method, where a continuous set of infinitesimal unitary transformations is applied in order to diagonalize the Hamiltonian perturbatively. This approach was established by WEGNER [114] in 1994 and independently by GzAzEK and WILSON [46] in 1993. A comprehensive introduction to the flow equation method is given in [60].

The general idea is to implement a sequence of infinitesimal unitary transformations such that

$$
\boldsymbol{H}(B+\mathrm{d} B)=e^{\boldsymbol{\eta}(B) \mathrm{d} B} \boldsymbol{H}(B) e^{-\boldsymbol{\eta}(B) \mathrm{d} B} .
$$


Here, $B \in[0, \infty)$ is the real-valued flow parameter that connects all the unitarily equivalent Hamiltonians $\boldsymbol{H}(B)$, and $\boldsymbol{\eta}(B)$ is the anti-hermitian generator of the flow that depends on the flow parameter $B$ as well. This sequence is described by the differential equation

$$
\frac{\mathrm{d} \boldsymbol{H}(B)}{\mathrm{d} B}=[\boldsymbol{\eta}(B), \boldsymbol{H}(B)]_{-},
$$

the so-called flow equation. Taking $H(B=0)$ as the initial Hamiltonian, the goal is to get a more and more energy-diagonal Hamiltonian throughout the flow. WeGNER showed in 1994 [114] that a Hamiltonian

$$
\boldsymbol{H}(B)=\boldsymbol{H}_{0}(B)+\boldsymbol{H}_{\text {int }}(B),
$$

with $\boldsymbol{H}_{0}$ being the diagonal and $\boldsymbol{H}_{\text {int }}$ being the off-diagonal part, is successively diagonalized by the canonical generator

$$
\boldsymbol{\eta}_{\text {can. }}(B) \stackrel{\text { def }}{=}\left[H_{0}(B), H_{\text {int }}(B)\right]_{-} .
$$

In the limit $B \rightarrow \infty$, the Hamiltonian $H(B)$ will be energy-diagonal. This means that $H_{\text {int }}(B=\infty)$ will consist of elastic many-particle scattering processes where the difference in one-particle energies is zero.

Typically and as we will see later, the coupling between eqs. (2.45) and (2.47) induces a growing number of higher and higher order interaction terms in the flowing Hamiltonian. Hence, a perturbative treatment is necessary, where the coupling constant of $\boldsymbol{H}_{\text {int }}$ is a suitable truncation parameter for the case of weak interaction.

\subsubsection{Transformation of observables}

The correlations between particles of the many-body system that are induced by interactions in the Hamiltonian are no longer encoded in the energy-diagonal Hamiltonian after the flow equation transformation. Instead, the correlations are described by the transformed observables, whose composite structure can get quite intricate. To calculate the transformation of observables, one has to solve the corresponding flow equation

$$
\frac{\mathrm{d} \boldsymbol{O}(B)}{\mathrm{d} B}=[\boldsymbol{\eta}(B), \boldsymbol{O}(B)]_{-} .
$$

Here, one also faces the problem of a growing number of interaction terms that makes it necessary to truncate after some order of the perturbation parameter.

\section{Successful applications of the flow equation method}

The flow equation scheme has been applied e.g. to the spin-boson model [51] and the dissipative harmonic oscillator [52], where the lack of secular terms in comparison to naive perturbation theory could be nicely demonstrated. A more advanced model of spinless fermions on a one-dimensional chain and a density-density interaction with tunable integrability breaking in the hopping term has also been analyzed with the flow equation method, which could be tested against the time-dependent density matrix renormalization group technique (tDMRG) [36]. Unlike the tDMRG method, the flow equation approach is not limited to one-dimensional 
models. In [84], the HubBard model in $d>1$ dimensions was studied, where the timeevolution of the momentum distribution function after a weak interaction quench was calculated (see sec. 4.1).

In this thesis, we also turn our attention towards the HubBard model in $d>1$. The goal is to go from one-particle functions (like the momentum distribution function analyzed in [84]) to the more advanced case of two-particle correlation functions. Therefore, we first provide a short introduction to the HUBBARD model in the next chapter. 


\section{Chapter 3}

\section{Interacting electrons on a lattice}

In condensed matter physics, one prime challenge is to describe the collective behavior of electrons in crystals, which can lead to emergent phenomena such as superconductivity or magnetism. While the fundamental interactions between the electrons (and the atomic nuclei of the crystal) are well-known, the resulting many-particle effects are hard to describe because the HILBERT space of the corresponding wave function grows expontentially with the particle number. Therefore, we will need effective models and further approximations if we want to tackle the problem of solving the SCHRÖDINGER equation for the many-body wave function. One of the most fundamental models for describing quantum correlation effects between electrons is the so-called HUBBARD model. It is a vast simplification of electronic dynamics in solids as it describes the electrons as moving on a discrete lattice with tunneling between neighboring lattice sites and the electric repulsion as short-ranged and hence only being effective between electrons on the same lattice site. However, this interaction already induces correlations. And though the HubBard model has many extensions (like e.g. nearest-neighbor interactions or next-nearest-neighbor tunneling), it is very useful in its simple form, because it describes correlations between electrons in a straightforward way. Further physical motivation and a mathematical definition of the HUBBARD model will be given in sec. 3.1 .

While this model can be written down for arbitrary dimensionalities of the lattice, actual calculations get the harder the higher the spatial dimension. But when taking the extreme limit of infinite spatial dimensions, things become simpler again [81, 89]. At first glance, an infinite-dimensional lattice may seem unphysical. However, this approach has provided a good description of real materials (e.g. transition metals and materials with $f$ electrons) in the framework of dynamical mean-field theory (DMFT) combined with the local density approximation (LDA) [73, 113]. As we will make use of this approximation for some calculations in this thesis, we shall present the main idea behind electrons in infinite spatial dimensions in sec. 3.2.

Finally, this chapter concludes with some references to how the HubBard model can be simulated in the lab using cold atom experiments in sec. 3.3 and how time-dependent phenomena in real correlated materials can be studied by time-resolved and angle-resolved photoemission spectroscopy (trARPES) in sec. 3.4. 


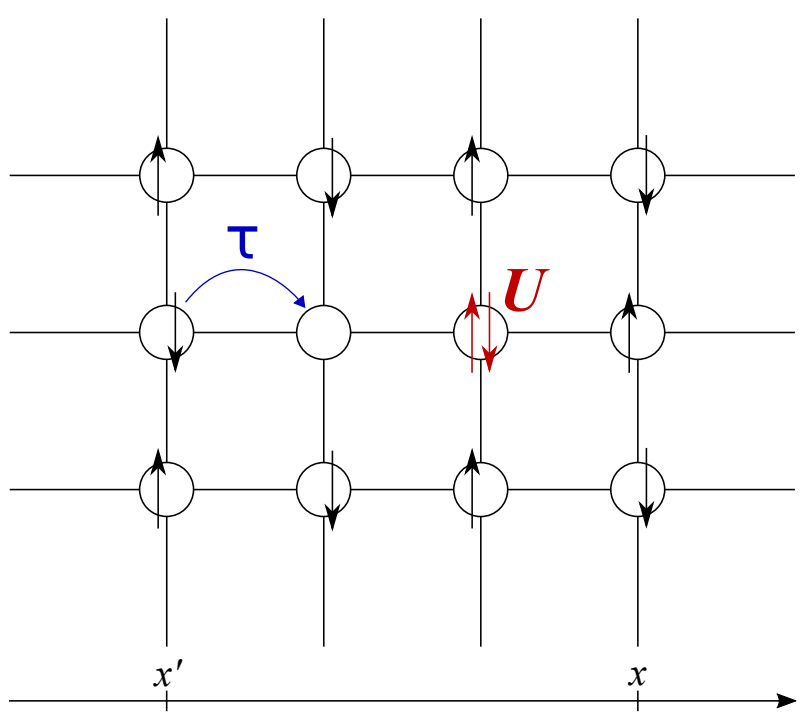

Figure 3.1: Illustration of the HubBARD model in real space in $d=2$ dimensions

\subsection{The HubBard model}

In 1963, HubBaRd introduced a simplified model to describe interacting electrons in narrow energy bands [55]. In a follow-up publication, he argued that his model contains a transition between a metallic and an insulating phase, which depends on the interaction strength [56]. This so-called metal-insulator transition was first discussed by Мотт in 1949 [87] and is therefore often called a MotT transition (for a review, see [88]). It is one of the main features of the HuBBARD model that made it so popular for studying strongly correlated electrons.

To define the HubBard model mathematically, we start by introducing the fermionic annihilation and creation operators $c_{k \sigma}, c_{k \sigma}^{\dagger}$ that depend on the momentum $k \in[-\pi, \pi]^{d}$ and the spin $\sigma \in\{\uparrow, \downarrow\}$. The momentum $k$ takes on discrete values given by the reciprocal lattice (with lattice dimension $d$ ). The fermionic character of the annihilation and creation operators is expressed in their canonical anti-commutation relations (CAR)

$$
\begin{aligned}
& {\left[c_{k \sigma}, c_{k^{\prime} \sigma^{\prime}}^{\dagger}\right]_{+} } \equiv \delta_{k, k^{\prime}} \delta_{\sigma, \sigma^{\prime}}, \\
& {\left[c_{k \sigma}, c_{k^{\prime} \sigma^{\prime}}\right]_{+} \equiv\left[c_{k \sigma^{\prime}}^{\dagger}, c_{k^{\prime} \sigma^{\prime}}^{\dagger}\right]_{+} \equiv 0, }
\end{aligned}
$$

where $[A, B]_{+} \equiv A B+B A$ is the anti-commutator.

The dynamics of the electrons that are described by the fermionic annihilation and creation operators is governed by two competing energy terms (cf. Fig. 3.1):

First, the electrons can tunnel between neighboring lattice sites $\langle i, j\rangle$, where the rate is given by the corresponding tunneling matrix element $\tau$. This is expressed by the kinetic Hamiltonian

$$
H_{0}=-\tau \sum_{\langle i, j\rangle, \sigma}\left(C_{i \sigma}^{\dagger} C_{j \sigma}+C_{j \sigma}^{\dagger} C_{i \sigma}\right)
$$


where

$$
\begin{aligned}
& C_{l \sigma}=\frac{1}{\sqrt{\Omega}} \sum_{k} e^{i(l \cdot k)} c_{k \sigma}, \\
& C_{l \sigma}^{\dagger}=\frac{1}{\sqrt{\Omega}} \sum_{k} e^{-i(l \cdot k)} c_{k \sigma}^{\dagger}
\end{aligned}
$$

are the annihilation and creation operators FOURIER transformed to real space, and $\Omega$ is the number of lattice sites. In momentum space, the kinetic Hamiltonian is simply

$$
H_{0}=\sum_{k, \sigma} \epsilon_{k} c_{k \sigma}^{\dagger} c_{k \sigma}
$$

with

$$
\epsilon_{k}=-2 \tau \sum_{i=1}^{d} \cos \left(k_{i}\right),
$$

being the dispersion relation and the momentum sum $\sum_{k}$ running over the reciprocal lattice points. In the following, we will use the kinetic Hamiltonian in momentum space from eq. (3.6) with a general dispersion relation $\epsilon_{k}$ which may differ from the specific one in eq. (3.7). The Fermi energy is defined as $\epsilon_{\mathrm{F}}=0$.

Secondly, two electrons repel each other if they are on the same lattice site $i$. Due to the PAULI principle, this can only affect electrons of different spin. This motivates the following form of the interaction Hamiltonian

$$
\begin{aligned}
\boldsymbol{H}_{\mathrm{int}} & =U \sum_{i} \boldsymbol{n}_{i \uparrow} \boldsymbol{n}_{i \downarrow} \\
& \equiv U \sum_{i} C_{i \uparrow}^{\dagger} C_{i \uparrow} C_{i \downarrow}^{\dagger} C_{i \downarrow},
\end{aligned}
$$

where $U$ is the strength of the on-site Coulomb interaction. In momentum space, this twoparticle interaction term looks like

$$
H_{\text {int }}=\frac{U}{\Omega} \sum_{k_{1}^{\prime}, k_{1}} \sum_{k_{2}^{\prime}, k_{2}} c_{k_{1}^{\prime} \uparrow}^{\dagger} c_{k_{1} \uparrow} c_{k_{2}^{\prime} \downarrow}^{\dagger} c_{k_{2} \downarrow} \delta_{k_{1}^{\prime}+k_{2}^{\prime}, k_{1}+k_{2}} .
$$

The form of the Hamiltonian $\boldsymbol{H}_{\text {int }}$ clarifies that the on-site interaction induces collision processes $\left(k_{1} \uparrow, k_{2} \downarrow\right) \rightarrow\left(k_{1}^{\prime} \uparrow, k_{2}^{\prime} \downarrow\right)$, where the total momentum is conserved, i.e., $k_{1}+k_{2}=$ $k_{1}^{\prime}+k_{2}^{\prime}$.

The full Hamiltonian

$$
\begin{aligned}
H & =H_{0}+H_{\text {int }} \\
& =\sum_{k, \sigma} \epsilon_{k} c_{k \sigma}^{\dagger} c_{k \sigma}+\frac{U}{\Omega} \sum_{k_{1}^{\prime}, k_{1}, k_{2}^{\prime}, k_{2}} c_{k_{1}^{\prime} \uparrow}^{\dagger} c_{k_{1} \uparrow} c_{k_{2}^{\prime} \downarrow}^{\dagger} c_{k_{2} \downarrow} \delta_{k_{1}^{\prime}+k_{2}^{\prime}, k_{1}+k_{2}}
\end{aligned}
$$

is just the Hubbard model, or Fermi-Hubbard model, in order to distinguish it from the bosonic analogue. The generality of $\epsilon_{k}$ allows us to describe arbitrary lattice structures, rather 
than being restricted to the simple cubic lattice depicted in Fig. (3.1) and described by the dispersion relation from eq. (3.7).

In one dimension, the FERMI-HuBBARD model is exactly solvable (for a textbook introduction we refer to [35]), while in higher dimensions no known general solution exists. This motivates the development of suitable approximation schemes like the perturbative treatment of the weakly interacting case (with $U \ll \tau$ ) that we will elaborate on in this thesis. Further simplifications can be made by using the limit of infinite spatial dimensions, presented in the following section.

\subsection{Limit of infinite spatial dimensions}

When we calculate expectation values of observables that are composed of the annihilation and creation operators introduced before, we are often left with sums over momenta, $\sum_{k}$, that are not easy to assess analytically, especially if we go to higher spatial dimensions. If the summands depend on the energies $\epsilon_{k}$ and the only explicit $k$-dependence is given by some momentum conservation constraint in form of a $\delta$-function, we can introduce one-dimensional energy integrals, $\int \mathrm{d} \epsilon \delta\left(\epsilon-\epsilon_{k}\right)$, which leaves us with some generalized density of states [112] that can, for example, look like

$$
D_{k}\left(\epsilon_{1}, \epsilon_{2}, \epsilon_{3}\right)=\frac{1}{\Omega^{3}} \sum_{k_{1}, k_{2}, k_{3}} \delta\left(\epsilon_{1}-\epsilon_{k_{1}}\right) \delta\left(\epsilon_{2}-\epsilon_{k_{2}}\right) \delta\left(\epsilon_{3}-\epsilon_{k_{3}}\right) \delta_{k+k_{3}, k_{1}+k_{2}} .
$$

So, the troublesome term in the calculation is the $k$-dependent $\delta$-function at the end. If we go to the extreme limit of infinite spatial dimensions, $d \rightarrow \infty$, however, this $\delta$-function turns into a constant factor and the whole expression can be written in form of one-dimensional energy integrals [81, 89]. An important prerequisite for the vanishing of the momentum conservation constraint $\delta_{k+k_{3}, k_{1}+k_{2}}$ is the rescaling of the HUBBARD model that needs to be done before taking the limit $d \rightarrow \infty$, which we will motivate in the following subsection.

\subsubsection{Rescaling of the HubBard model}

When looking at the dispersion relation from eq. (3.7), $\epsilon_{k}=-2 \tau \sum_{i=1}^{d} \cos \left(k_{i}\right)$, the corresponding density of states is given by

$$
D(E) \equiv \frac{1}{\Omega} \sum_{k} \delta\left(E-\epsilon_{k}\right) .
$$

In the limit $d \rightarrow \infty$, the central limit theorem yields

$$
D(E) \stackrel{d \rightarrow \infty}{=} \frac{1}{\sqrt{2 \pi} \cdot \tau \sqrt{2 d}} \exp \left(-\frac{1}{2}\left(\frac{E}{\tau \sqrt{2 d}}\right)^{2}\right)
$$

which is just a Gaussian profile with a width $\sigma=\tau \sqrt{2 d}$. But this means that in infinite spatial dimensions the density of states becomes infinitly broad and flat, and hence unphysical [112]. Therefore, we need to rescale

$$
\tau=\frac{\tau^{*}}{\sqrt{2 d}}
$$


with finite $\tau^{*}$, in order to get a meaningful density of states [81]. Only with this rescaling, the interplay between kinetic energy and on-site interaction in the HUBBARD Hamiltonian from eq. (3.10) is balanced and of the same order of magnitude in infinite dimensions.

\subsubsection{Simplifications in infinite dimensions}

We have already mentioned that calculations of expectation values simplify in the limit of infinite spatial dimensions. If we take, for example, the generalized density of states from eq. (3.11),

$$
D_{k}\left(\epsilon_{1}, \epsilon_{2}, \epsilon_{3}\right)=\frac{1}{\Omega^{3}} \sum_{k_{1}, k_{2}, k_{3}} \delta\left(\epsilon_{1}-\epsilon_{k_{1}}\right) \delta\left(\epsilon_{2}-\epsilon_{k_{2}}\right) \delta\left(\epsilon_{3}-\epsilon_{k_{3}}\right) \delta_{k+k_{3}, k_{1}+k_{2}},
$$

we can replace the $\delta$-function by a constant $\frac{1}{\Omega}$, and hence the explicit $k$-dependence vanishes [112]. For a detailed derivation, we refer to [89].

Thus, in infinite spatial dimension, the generalized density of states factorizes into singleparticle densities,

$$
\begin{aligned}
D_{k}\left(\epsilon_{1}, \epsilon_{2}, \epsilon_{3}\right) & \stackrel{d \rightarrow \infty}{=} \frac{1}{\Omega^{4}} \sum_{k_{1}, k_{2}, k_{3}} \delta\left(\epsilon_{1}-\epsilon_{k_{1}}\right) \delta\left(\epsilon_{2}-\epsilon_{k_{2}}\right) \delta\left(\epsilon_{3}-\epsilon_{k_{3}}\right) \\
& =\frac{1}{\Omega} D\left(\epsilon_{1}\right) D\left(\epsilon_{2}\right) D\left(\epsilon_{3}\right) .
\end{aligned}
$$

Now, the complicated sums over momenta can be fully replaced by one-dimensional energy integrals.

\subsubsection{Physical justification of the limit}

The limit of infinite spatial dimensions may seem unrealistic with regard to real materials. However, it maintains the two important features of the HUBBARD model that we are interested in: First, we still have a discrete lattice structure. Secondly, with the rescaling, we have a balanced contribution of kinetic energy and on-site interaction. Since these are the features whose dynamical implications we want to study, the limit $d \rightarrow \infty$ seems useful.

For instance, the previously mentioned metal-insulator transition was first formally shown to be present in the HUBBARD model in the infinite-dimensional limit [102]. The critical parameter of the transition is the ratio $U / \tau$, where for small $U$ the kinetic energy dominates the dynamics and thus the model is in the metallic phase. If one increases the interaction strength $U$ above some critical value, localization effects will push the model into the insulating phase.

In dynamical mean-field theory one also uses the rescaling for high dimensions. In fact, not the real spatial dimension $d$ but the lattice coordination number $Z$ is the large quantity there. The first order correction, when going to finite $Z$, is then given by $O\left(\frac{1}{Z}\right)$ [112]. For a facecentered cubic lattice, where $\frac{1}{Z}=\frac{1}{12}$, this correction is already small. Hence, this may serve as another justification of the seemingly unrealistic limit of infinite spatial dimensions or coordination number. Indeed, DMFT + LDA methods have been very successful in describing real materials [73, 113]. 


\subsection{Experiments with cold atoms}

Probing the dynamics of isolated quantum many-body systems like the HuBBARD model in experiments is very challenging because of the difficulties that lie in creating systems that are isolated from the environment and at the same time accessible for measurements. An ideal playground that meets these two criteria is found in experiments with cold atoms, where model systems are simulated by atoms trapped in optical lattices [8]. These experiments also allow for a well-controlled tuning of model parameters, such as the hopping rate or the interaction strength in the HuBBARD model.

After equilibrium properties of many-body systems have extensively been studied in cold atoms [9], recent progress has been made in observing unitary dynamics [49, 61], which has opened up the field for studying nonequilibrium settings. When it comes to dynamical observations, the advantage of cold atomic gases lies in their long coherence times due to their isolation from the environment. These coherence times can reach several seconds, whereas the dynamical relevant time scales of the atoms are much shorter, which allows for resolving the atoms' intrinsic dynamics [72].

\subsubsection{Experimental setup}

\section{Optical lattices}

The potential landscape of periodic many-body systems can be simulated in the lab by interfering laser beams. When the lasers are arranged in a way that their electric field forms a standing wave, the oscillating field at the anti-nodes induces a likewise oscillating dipole moment on an atom that is released into this field. The dipole moment itself interacts with the laser field again, which provides an optical trap for the atom. The dipole potential has the same periodicity as the standing laser wave, which thereby forms an optical lattice for the atom. Such an optical lattice can be generated in one, two or three dimensions [8].

The lattice constant, i.e., the distance between neighboring lattice sites, as well as the depth of the lattice potential can be tuned - also dynamically - with high precision by varying the frequency or the intensity of the laser. Twisting the lasers against each other even enables the generation of different lattice geometries going e.g. from simple cubic to hexagonal systems [94].

\section{Measurements}

When one releases dilute atomic gases into the optical lattice, they are cooled down to very low temperatures by the laser trapping. Individual atoms can be tracked e.g. by fluorescence imaging [103] or scanning electron microscopy [44], and hence site-resolved measurements become possible [5, 107]. This typically corresponds to a measurement of the density distribution $n(x)$ in real space. By time-of-flight expansions, where one suddenly turns off the optical lattice and measures the particle positions after a certain amount of time, one has also access to the momentum distribution $n(k)$ in FOURIER space [2, 29]. As one disrupts the atomic gas during these measurements, they must be repeated for equally prepared systems at different delay times in order to study dynamical features.

Beyond fluorescence imaging and scanning tunneling microscopy one can also use matterwave interference, which enables the measurement of observables that do not only depend 
a
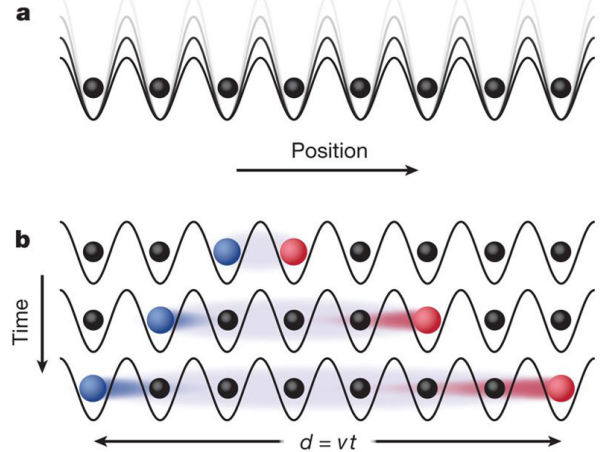

Quench

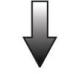

bosonic atom before the quench. b: After lowering the lattice depth, entangled pairs of doublons (red) and holons (blue) emerge and start moving apart with a relative velocity $v$. [Reprinted by permission from Springer Nature Customer Service Centre GmbH: Springer Nature, Nature 481 ("Light-cone-like spreading of correlations in a quantum many-body system", ChENEAU et al.) (c) 2012]

on the absolute value of the quantum field (like the density and momentum distributions) but also on the complex phase [50, 104].

\section{Modeling of interactions}

The high controllability of the optical lattice allows for inducing nonequilibrium dynamics e.g. by implementing a quantum quench after the atoms have reached a certain distribution in the lattice. Tuning the depth of the lattice potential, for example, influences the tunneling rate for atoms between neighboring lattice sites. An important feature of cold atom experiments is that even interactions between particles (like the on-site repulsion of the HUBBARD model) can be simulated using FESHBACH resonances [37, 40].

When two atoms come close to one another, their valence electrons interact with each other and induce a scattering process. By applying an external magnetic field, one can tune the scattering length, which drastically changes if the state of the two atoms comes close to a molecular bound state, a so-called FESHBACH resonance. Now, one utilizes that on the one hand the scattering length depends on the external magnetic field, while on the other hand it is directly related to the two-particle interaction potential. Thereby, one is able to freely tune the interaction strength between the particles and even change the sign, going from repulsive also to attractive interactions [26].

\subsubsection{Spatial correlation functions}

An experiment that comes close to what we will study in this thesis - namely the spreading of two-particle correlations in the HUBBARD model out of equilibrium - was carried out by CHENEAU et al. and published in 2012 [24].

They created a BOSE-EINSTEIN condensate in an effectively one-dimensional optical lattice hence simulating the BOSE-HUBBARD model - where initally each lattice site was filled with $\bar{n}=1$ atom and the total number of lattice sites ranged between 10 and 18. A good control of the lattice potential depth let them tune the model parameters $J$ (hopping parameter) and $U$ (interaction strength) of the Bose-HubBard model: A deep optical lattice corresponds to a large $U / J$ because hopping between lattice sites becomes more difficult.

The initial state was prepared deep in the Mотт-insulating phase with $U / J=40$ and at low temperature $k_{\mathrm{B}} T \approx 0.1 U$. A sudden lowering of the lattice depth, which corresponded to a quantum quench to $U / J=9$ (and subsequently also to $U / J=7$ and $U / J=5$ ), brought the system out of equilibrium (see Fig. 3.2). After a variable amount of time they froze the 
Figure 3.3: Time evolution of the twopoint parity correlations. The correlation peak propagates through the system with increasing time. main figure: Experimental data (open circles) for a quench from $U / J=40$ to $U / J=9$ and numerical data (green line) for an infinite system at zero temperature as well as analytical prediction (dashed black line). small figure: Experimental data in a color map that demonstrates the lightcone-like behavior. [Reprinted by permission from Springer Nature Customer Service Centre GmbH: Springer Nature, Nature 481 ("Light-cone-like spreading of correlations in a quantum many-body system", CHENEAU et al.) (C) 2012]

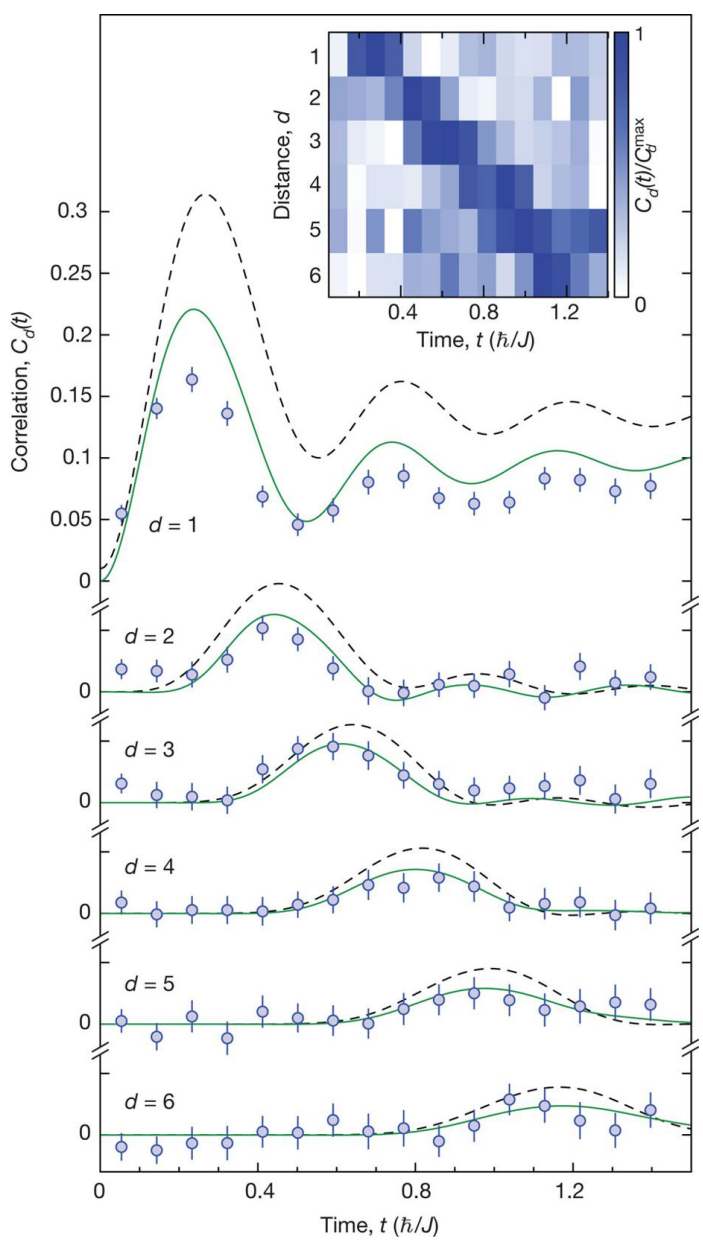

density distribution by drastically increasing the lattice potential depth. In this state, the siteresolved occupation number was measured with fluorescence imaging.

By repeated measurements, they were able to extract the two-point parity correlation function

$$
C_{d}(t)=\left\langle\boldsymbol{s}_{j}(t) \boldsymbol{s}_{j+d}(t)\right\rangle-\left\langle\boldsymbol{s}_{j}(t)\right\rangle\left\langle\boldsymbol{s}_{j+d}(t)\right\rangle
$$

with $\boldsymbol{s}_{j}(t)=e^{i \pi\left(n_{j}-\bar{n}\right)}$ being the parity of the occupation number $\boldsymbol{n}_{j}$ of bosonic atoms at lattice site $j$.

As a result they found that the spatial correlation function has a certain peak that propagates in time (see Fig. 3.3). From this they calculated the corresponding quasi-particle velocity, which was compared to numerical results for an infinite lattice, performed with the density matrix renormalization group (DMRG) method. It was found that the measurements and numerical results were in good agreement.

The interesting physical behavior that was observed is that the propagation of quasi-particles always stays below some maximal and finite velocity. In other words: the propagation is restricted to an effective light cone due to causality. We shall return to this issue in sec. 4.2.

\subsubsection{Experiments with fermions}

While the experiment by CHENEAU et al., described above, was carried out with bosons, quantum simulations of the HubBard model are also possible with fermions. 
One of the first experiments was reported on by Modugno et al. in 2003 [83]. They prepared fermions in a one-dimensional optical lattice and measured the momentum distribution for different lattice constants. They found that for very tight lattice configurations the system's density of states changed to describe a quasi-two-dimensional FERMI gas.

A further experiment by KöHL et al. from 2005 [64] simulated the attractive single-band Fermi-Hubbard Hamiltonian in a three-dimensional lattice, while the repulsive analogue could be simulated by JöRDENS et al. in 2008 [58].

Finally, PARsons et al. showed in 2016 [92] that they can assess the spatial spin correlation function in the two-dimensional Fermi-HubBard model (in the MotT-insulating regime at $U / \tau=8$ ). They performed their measurements at different low temperatures and found excellent agreement with results from the numerical linked-cluster expansion (NLCE) under a local density approximation (LDA).

\subsection{Time-resolved ARPES}

Experiments with cold atoms in optical lattices are kind of artificial because in the end we want to investigate the behavior of electrons in real materials. This can be done by angle-resolved photoemission spectroscopy (ARPES), which is the state-of-the-art method to measure the electronic band structure $\epsilon(k)$ or momentum distribution $n(k)$ in correlated materials that are e.g. described by the HuBBARD model.

Photoemission spectroscopy is based on the photoelectric effect that was discovered by HERTZ in 1887 [54] and explained by EINSTEIN in 1905 [34]: Shining light on a solid may lead to the emission of electrons from its surface because the photons transfer their energy onto the electrons.

When the energy $\hbar \omega$ of the photon is high enough to drive an electron out of the material, the emitted electron will have a kinetic energy

$$
E_{\mathrm{kin}}=\hbar \omega-E_{\mathrm{B}}-\phi
$$

where $E_{\mathrm{B}}$ is the binding energy of the electron and $\phi$ is the electron work function, a constant that depends on the surface of the material [69]. The work function needs to be calibrated by comparison with a well known sample (like gold or copper). Then, by measuring $E_{\text {kin }}$ with a detector one can reconstruct the energy $E_{\mathrm{B}}$ of the former core or valence electron. The density of states of the valence electrons at a certain energy $E_{\mathrm{B}}$ will be proportional to the number of photoelectrons emitted with the energy $E_{\text {kin }}$.

In photoemission experiments, one distinguishes between x-ray photoelectron spectroscopy (XPS), which is needed to emit core level electrons, and ultraviolet photoelectron spectroscopy (UPS), which is sufficient for emission of valence electrons.

\subsubsection{Angle-resolved photoemission spectroscopy}

Photoemission spectroscopy allows for measuring the density of states of surface electrons. In order to study the full band structure $\epsilon(k)$, one needs to measure also the emission angle of the photoelectrons. The polar angle $\theta$, the azimuthal angle $\varphi$ and the kinetic energy $E_{\text {kin }}$ 
define the wave vector $K$ of the emitted electron,

$$
K=\frac{\sqrt{2 m_{\mathrm{e}} E_{\mathrm{kin}}}}{\hbar}\left(\begin{array}{c}
\sin \theta \cos \varphi \\
\sin \theta \sin \varphi \\
\cos \theta
\end{array}\right) .
$$

Now, the question arises how this vector $K$ of the emitted electron is related to the former momentum $\hbar k$ of the electron inside the solid. Because the momentum of the photon is much smaller than the momenta of involved electrons, we can simply neglect it in the momentum balance. The momentum component parallel to the surface of the sample is conserved due to the translational invariance inside and outside the sample. This means that

$$
k_{\|}=K_{\|}
$$

For two-dimensional crystals, there is no perpendicular component $k_{\perp}$, which makes it easy to measure $\hbar k$.

For three-dimensional materials, we need to determine $k_{\perp}$ as well. This can be done in the so-called sudden approximation framework, for which we refer to [69].

The momentum- and energy-dependent spectral function $A(k, \epsilon)$ that is measured with ARPES not only determines the band structure $\epsilon(k)$ but also the momentum distribution function $n(k)$ via the relation

$$
n(k)=\int \mathrm{d} \epsilon f(\epsilon) A(k, \epsilon),
$$

where $f(\epsilon)$ is the FERMI-Dirac distribution.

An interesting question is how the momentum distribution function changes in time when the system is driven out of equilibrium. Such scenarios can be studied by time-resolved measurements with pump-probe spectroscopy.

\subsubsection{Pump-probe spectroscopy}

The possibility to create ultrashort laser pulses in the range of femtoseconds [45] has opened up the field of pump-probe spectroscopy, where one can perform time-dependent measurements in materials driven out of equilibrium.

The general idea is to first pump energy into a system with an ultra-short laser pulse. This excitation will induce nonequilibrium dynamics in the sample. After a certain delay time, a second so-called probe pulse is sent to induce a photoemission spectrum. For example, one can measure the electronic spectral function $A(k, \epsilon)$ via ARPES. By varying the delay time $t$, multiple measurements of the photoemission spectrum finally reveal its time-dependence, $A(k, \epsilon, t)$, where the resolution can reach the order of femtoseconds.

This so-called time-resolved ARPES (trARPES) has turned out to be a very useful tool to study dynamical processes in systems with correlated electrons.

For example, in an experiment by PERfeTti et al. from 2006, a photo-induced phase transition from an insulator to a metal was discovered [93]. The sample was the MотT-insulating material $1 T-\mathrm{TaS}_{2}$ that was excited and brought out of equilibrium by a pump laser. Then, 

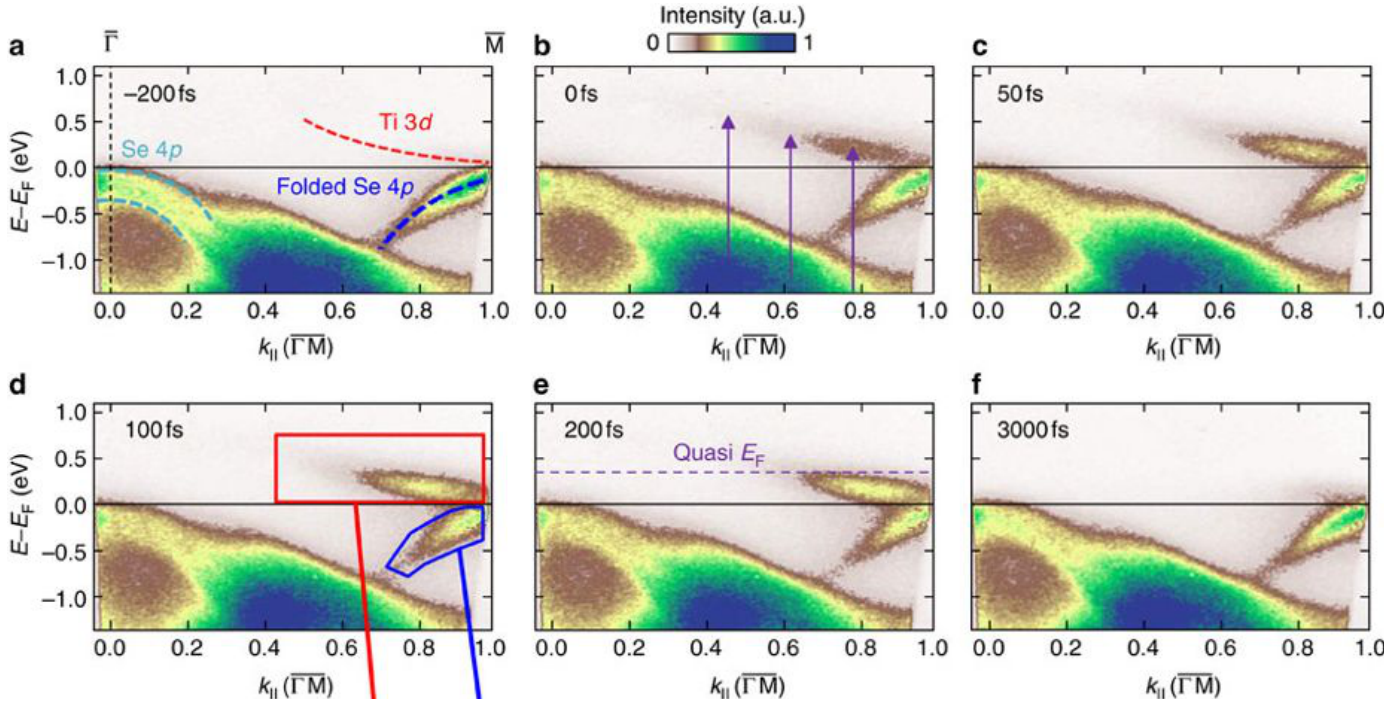

Figure 3.4: Spectra of the transient electronic dynamics of photoexcited $1 T-\mathrm{TiSe}_{2}$. The red area shows the ultra-fast accumulation of electrons in the Ti $3 d$ band. [Reprinted by permission from Creative Commons Attribution 4.0 International License Nature Communications 7 ("Self-amplified photo-induced gap quenching in a correlated electron material", MATHIAS et al.) (c) 2016. Only part of the original figure is shown.]

PERfetTi et al. measured the spectral function with trARPES and found that the electronic band gap of the Mотт insulator closes within $100 \mathrm{fs}$ after the excitation, which corresponds to a phase transition to the metallic phase. Accompanying DMFT calculations confirmed that this process is solely driven by the electrons and not by a distortion of the lattice.

In 2016, Mathias et al. followed up with a similar experiment on $1 T-\mathrm{TiSe}_{2}$ [79]. They asked the question whether there is a universal relation between the size of the energy gap in the correlated material and the relaxation dynamics of the electrons.

Mathias et al. cooled down the material to the temperature of liquid nitrogen, at which $1 T-\mathrm{TiSe}_{2}$ is in the charge density wave (CDW) phase and has a characteristic energy gap $\sim 100 \mathrm{meV}$. After pumping energy into the system, they measured the photoemission spectra shown in Fig. 3.4. The energy gap between the occupied Se $4 p$ and the unoccupied Ti $3 d$ bands closed extremely fast after intense optical excitation. They explained this by a positive feedback effect: The carrier multiplication becomes faster with a decreasing energy gap.

A combined method of time-resolved x-ray diffraction and time-resolved ARPES was established by GERBER et al. in 2017 [43]. While x-ray diffraction allows for tracking the lattice dynamics, a simultaneous usage of both techniques enabled them to study the electron-phonon coupling in iron-based superconductors. This coupling can be enhanced by electronic correlations, as they found in their experiment.

\section{Comparison to cold atom experiments}

When studying the nonequilibrium behavior of correlated electrons, trARPES deals with realistic materials while simulations with cold atoms are more artificial. A main difference is that the samples in trARPES can be considered as open quantum systems that interact with the environment. In contrast, cold atoms on optical lattices can be considered as isolated systems for sufficiently long times. Another difference is that samples in trARPES are driven 
out of equilibrium by local photoexcitations, while in quantum simulations the usual setup is a global quantum quench.

One also has to be aware of that one cannot directly measure correlation functions with trARPES like in the cold atom experiment by CHENEAU et al. (see sec. 3.3.2). In this thesis, we will study the general time evolution of fermionic operators in the HUBBARD model. Our results can be used for the calculation of a wide range of observables that may be accessible either in trARPES measurements or cold atom experiments. 


\section{Chapter 4}

\section{Nonequilibrium phenomena}

In isolated quantum systems out of equilibrium, it is not entirely clear how equilibrium or thermalization is reached under a unitary time evolution.

Integrable systems do not thermalize at all because there are too many constants of motion that constrain the dynamics and prevent chaotic motion. Actually, nonthermal steady states in integrable systems are found in analytical calculations [31] as well as in experiments with cold atoms [61].

While nonintegrable systems thermalize in the sense that some observables reach equilibrium values that can be predicted by a thermal ensemble [97], an interesting question is what happens to systems whose integrability is weakly broken, e.g. by a small perturbation. Here, one observes a phenomenon called prethermalization that will be addressed in sec. 4.1.

To analyze the dynamics of equilibration and thermalization, one ascertains the speed of information that propagates through the system by considering the build-up of correlations. Their spatiotemporal structure is often confined to an effective light cone, which will be clarified in sec. 4.2 .

\subsection{Prethermalization}

In 2004, BERGES et al. discussed the concept of prethermalization in complex quantum systems far from equilibrium in the context of heavy-ion collisions [7]. When a quantum system is driven out of equilibrium, it can happen that on an intermediate time scale - long before thermalization sets in - certain bulk quantities already equilibrate to a prethermal value while momentum-dependent mode quantities are still far from equilibrium. As an example for such a bulk quantity they considered the equation of state $p(\epsilon)$, i.e., the relation between pressure $p$ and energy density $\epsilon$. When this function is almost constant in the intermediate prethermalization regime, a closed hydrodynamical description becomes possible. At the same time, mode quantities like the momentum distribution will still memorize the initial conditions and only equilibrate on a longer time scale.

\subsubsection{Time scale separation}

Following the publication of Berges et al., MoecKeL and KeHreIn studied such a mode quantity in the context of condensed matter physics [ $[84,85,86]$. They considered the time evolution of the momentum distribution function in the HUBBARD model out of equilibrium (see 
sec. 3.1).

The initial state was the zero-temperature ground state of the noninteracting Hamiltonian, i.e., the FERMI sea. At time $t=0$, they implemented a weak interaction quench and let the initial state evolve in time under the interacting Hamiltonian. By means of unitary perturbation theory (see sec. 2.2.1), they calculated the momentum distribution function in a second-order expansion in the interaction strength $U$ and got the expression

$$
\begin{aligned}
\Delta N_{k}^{\mathrm{NEQ}}(t) & \stackrel{\text { def }}{=}\left\langle\boldsymbol{c}_{k \uparrow}^{\dagger}(t) \boldsymbol{c}_{k \uparrow}(t)\right\rangle-\left\langle\boldsymbol{c}_{k \uparrow}^{\dagger}(0) \boldsymbol{c}_{k \uparrow}(0)\right\rangle \\
& =-4 U^{2} \int_{-\infty}^{\infty} \mathrm{d} E \frac{\sin ^{2}\left(\frac{1}{2}\left(E-\epsilon_{k}\right) t\right)}{\left(E-\epsilon_{k}\right)^{2}} I_{k}\left(E, n_{k}\right)+O\left(U^{3}\right),
\end{aligned}
$$

with $I_{k}\left(E, n_{k}\right)$ being some phase space factor that defines the bound of integration and that depends on the initial momentum distribution $n_{k}$. The perturbative result is stable up to and including the prethermalization time scale $t \lesssim \rho_{\mathrm{F}}^{-1} U^{-2}$, where $\rho_{\mathrm{F}}$ is the density of states at the FERMi level.

Then, Moeckel and KeHrein worked out the momentum distribution function of the interacting HubBard model in equilibrium and at zero temperature, $\Delta N_{k}^{\mathrm{EQ}}$, and compared it to the time average $\overline{\Delta N_{k}^{\mathrm{NEQ}}}$ of the nonequilibrium function from eq. 4.1 . As the time average only refers to the prethermalization regime, it is exactly the prethermal value. They found that

$$
\overline{\Delta N_{k}^{\mathrm{NEQ}}}=2 \Delta N_{k}^{\mathrm{EQ}}+O\left(U^{3}\right) .
$$

This means that the prethermal value of the momentum distribution after a weak quench differs from the equilibrium value at zero temperature by a factor 2 .

By means of the HELLMANN-FEYNMAN theorem, they established respective relations for the kinetic energy $E_{\text {kin }}=\left\langle\boldsymbol{H}_{0}\right\rangle$ and the interaction energy $E_{\text {int }}=\left\langle\boldsymbol{H}_{\text {int }}\right\rangle$,

$$
\begin{aligned}
& \overline{E_{\mathrm{kin}}^{\mathrm{NEQ}}}=2 E_{\mathrm{kin}}^{\mathrm{EQ}}+O\left(U^{3}\right), \\
& \overline{E_{\mathrm{int}}^{\mathrm{NEQ}}}=E_{\mathrm{int}}^{\mathrm{EQ}}+O\left(U^{3}\right) .
\end{aligned}
$$

MOECKeL and KeHREIN concluded that the nonequilibrium momentum distribution function and the kinetic energy do not reach their zero-temperature equilibrium values within the prethermalization regime, while the interaction energy does. This fits in the picture by BERGES et al. because the momentum distribution function is a mode quantity, while the interaction energy is a sum over local terms.

MoeCKel and KeHREIN argued that thermalization sets in on a much longer time scale $t \gtrsim$ $\rho_{\mathrm{F}}^{-3} U^{-4}$, which is described by the quantum BolTzMAnN equation. Here, the heating effect of the quench increases the temperature to $T \propto U$ and the above relations, where the equilibrium values are associated with zero temperature, no longer hold. 


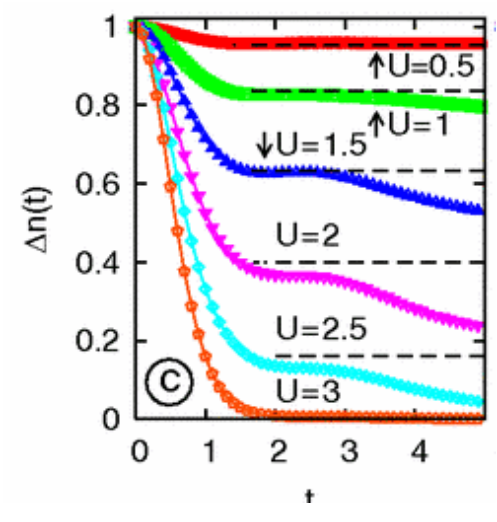

Figure 4.1: Momentum distribution function after quenches to $U \leq 3$. Comparison of DMFT data (color lines) to UPT prediction of prethermalization values (dashed lines). [Reprinted figure with permission from ECKSTEIN et al. Physical Review Letters 103 (2009), 056403 Copyright 2009 by the American Physical Society. Only part of the original figure is

To sum up, Moeckel and KeHreIN identified three separated time regimes after the interaction quech:

(i) $0 \lesssim t \lesssim \rho_{\mathrm{F}}^{-1} U^{-2}$ : formation of quasi-particles,

(ii) $\rho_{\mathrm{F}}^{-1} U^{-2} \lesssim t \lesssim \rho_{\mathrm{F}}^{-3} U^{-4}$ : quasi-steady prethermalization regime,

(iii) $\rho_{\mathrm{F}}^{-3} U^{-4} \lesssim t$ : thermalization regime.

If one decreases the interaction strength $U$ and comes closer to integrability, thermalization will be massively delayed. This fits to the picture that integrable systems do not thermalize. The analytical findings by MOECKEL and KEHREIN were numerically confirmed by ECKSTEIN, KOLLAR and WERNER, who performed their calculations in the limit of dynamical mean-field theory (DMFT) [32, 33]. A comparison of the DMFT results and the analytical prethermalization values is shown in Fig. 4.1. Here, one also sees that the prethermalization plateaus become broader with decreasing interaction strength.

\subsubsection{Generalized Gibbs ensemble}

The prethermalization value of the momentum distribution function from eq. (4.2) differs from the equilibrium value and is therefore not related to the thermal density matrix $\boldsymbol{\rho}_{\text {th. }}$. However, Kollar et al. demonstrated in a follow-up publication that expectation values for prethermalized states can be predicted by a generalized Gibbs ensemble (GGE) [65], which originates from the description of nonthermal steady states in integrable systems [100]. Hence, prethermalization plateaus are illustrated as near-integrability induced bottlenecks in the thermalization dynamics.

Indeed prethermalization is a universal concept in weakly perturbed systems that not only applies to condensed matter systems but also e.g. to the reheating of the post-inflationary universe [70, 96, 99].

Following the argument of Kollar et al., let us consider a weakly perturbed system $\boldsymbol{H}=$ $H_{0}+g H_{\text {int }}$ that is close to integrability, i.e., $g \ll 1$. Then, the exactly solvable part of the Hamiltonian can be written as

$$
H_{0}=\sum_{\alpha} \epsilon_{\alpha} I_{\alpha}
$$


where $I_{\alpha}$ are nearly conserved quantities in the perturbed system. For the HuBBARD model from eq. 3.10), for example, we have $\boldsymbol{I}_{\alpha}=\boldsymbol{c}_{k \sigma}^{\dagger} \boldsymbol{c}_{k \sigma}$. If one replaces the thermal ensemble from eq. (1.2) by the so-called generalized Gibbs ensemble

$$
\begin{aligned}
\boldsymbol{\rho}_{\mathrm{GGE}} & =\frac{1}{Z} e^{-\sum_{\alpha} \lambda_{\alpha} I_{\alpha}}, \\
Z & =\operatorname{Tr}\left[e^{-\sum_{\alpha} \lambda_{\alpha} I_{\alpha}}\right],
\end{aligned}
$$

with some generalized inverse temperatures $\lambda_{\alpha}$, expectation values of prethermalized quantities are obtained by

$$
\langle O\rangle=\operatorname{Tr}\left[\rho_{\mathrm{GGE}} O\right] .
$$

The predictability of prethermalized expectation values by means of a GGE has been confirmed both in experiments [50, 108] and corresponding analytical calculations [63].

\section{Many-body localization}

While weakly perturbed systems will thermalize when they are tuned away from the integrable point, many-body localized states provide an alternative to study nonthermal steady states that are described by a GGE [111].

Many-body localization occurs in disordered systems and was first proposed by ANDERSON in 1958 [3], who considered free fermions in a random local potential,

$$
H=-\tau \sum_{i}\left(c_{i+1}^{\dagger} c_{i}+\text { h.c. }\right)+\sum_{i} \mu_{i} n_{i}
$$

He showed that the disorder of the random potential localizes all single-particle wave functions in space. Because excitations cannot move and transport energy through the system, thermalization fails. Interestingly, this is also the case for nonintegrable systems with disorder. An underlying reason is that the excited eigenstates satisfy an area law for the entanglement entropy [6, 106], which violates the eigenstate thermalization hypothesis (ETH).

In the many-body localized phase, there is an infinite number of local conserved quantities, so that one can speak of local integrability [106].

The appearance of many-body localization was recently confirmed in experiments with cold Fermi gases in optical lattices [66]. 


\subsection{LIEB-ROBINSON bounds}

The prethermalization behavior in the HUBBARD model has been analyzed for the momentum distribution function and now the natural question arises how correlations between particles build up after the interaction quench, which will be examined in this thesis.

In 1972, LIEB and ROBINSON evinced that information in quantum spin systems can only propagate with a finite group velocity $v$ [74]. The considered a general quantum lattice system with only local interactions in the Hamiltonian. For any two local observables $\boldsymbol{O}_{X}$ and $\boldsymbol{O}_{Y}$ that are defined over two disjoint sets $X$ and $Y$, one can derive the following bound for the commutator,

$$
\left\|\left[O_{X}(t), O_{Y}(0)\right]_{-}\right\| \leq c \min (|X|,|Y|)\left\|O _ { X } \left|\left\||| O_{Y}\right\| \exp \left(-\frac{\operatorname{dist}(X, Y)-v|t|}{\xi}\right) .\right.\right.
$$

In this notation that follows the lecture notes by Hastings [53], $\|\cdot\|$ is the operator norm, $c$ and $\xi$ depend on the type of interactions and the lattice geometry, $\operatorname{dist}(X, Y)$ measures the distance between $X$ and $Y$ and $v$ is a characteristic velocity.

The interpretation of this bound becomes clear, when we look at the argument of the exponential. If $\operatorname{dist}(X, Y)$ is larger than $v|t|$, the upper limit of the bound will decay exponentially in the spatial distance at fixed time $t$. This leads to the notion of an effective light cone, where the speed of light is replaced by the group velocity $v$. The commutator from eq. (4.10) only has relevant contributions inside the effective light cone and becomes exponentially small outside because the latter region is causally disconnected if $v$ is the maximum speed for spreading of information.

While the original proof by LIEB and ROBINSON is only valid for translational invariant spin systems with a finite HILBERT space, more recent versions also include lattice bosons and infinite HILBERT spaces [90].

\subsubsection{Connection to linear response theory}

It turns out that the commutator from eq. (4.10) also appears in the KuBo formula and is closely connected to linear response theory [68].

For a Hamiltonian

$$
H(t)=H_{0}+\Theta(t) V(t)
$$

that is weakly perturbed by some potential $V(t)$ for times $t \geq 0$ (here, $\Theta(t)$ is the HeAvyside step function), the time evolution of an observable $A$ can be estimated in a linear expansion in $V$ as

$$
\langle A(t)\rangle \approx\langle A(0)\rangle-i \int_{0}^{t} \mathrm{~d} t^{\prime}\left\langle\left[A(t), V\left(t^{\prime}\right)\right]_{-}\right\rangle
$$

Assuming a singular perturbation $V(t)=\delta(t) \boldsymbol{B}(t)$, this motivates the notion of the susceptibility

$$
\chi_{A, B}(t)=-i\left\langle[A(t), B(0)]_{-}\right\rangle .
$$

It is the linear response of the observable $A$ to a small perturbation $B$. For local operators $A$ and $B$, this susceptibility is subject to the Lieb-Robinson bound from eq. (4.10). It means 
Figure 4.2: Spatiotemporal behavior of the spin susceptibility (commutator) in the KoNDO model at zero temperature. The commutator vanishes exactly outside the effective light cone. [Reprinted figure with permission from Medvedyeva et al. Physical Review B 88 (2013), 094306 Copyright 2013 by the American Physical Society. Only part of the original figure is shown.]

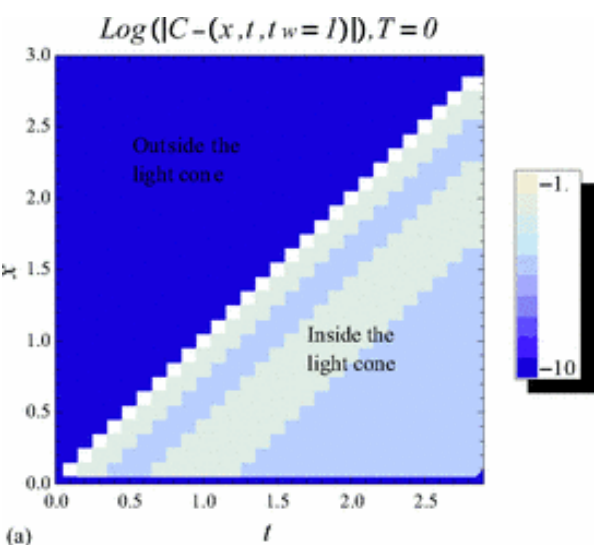

that the linear response only propagates with a finite velocity and that no response is given in causally disconnected regions.

This was shown for the spin susceptibility in the Kondo model by Medvedyeva et al. using exact results at the integrable point of the model [80]. In Fig. 4.2, it is shown how the susceptibility vanishes exactly outside an effective light cone.

It is important to note that the original LIEB-ROBINSON bounds are only formulated as bounds on the commutator, i.e., on response functions. In equilibrium, these are directly connected to correlation functions, i.e., anticommutators, via the fluctuation-dissipation relation. However, this relation is no longer exact in nonequilibrium settings [105].

\subsubsection{Equal-time connected correlation functions}

In current experiments with cold atoms, one can measure correlation functions (cf. Figs. 3.2 and 3.3 [24], which motivates further analysis on respective bounds for the equal-time connected correlation function of two observables $A$ and $B$, which is defined as

$$
C_{A, B}(t)=\langle A(t) B(t)\rangle_{\mathrm{c}} \equiv\langle A(t) B(t)\rangle-\langle A(t)\rangle\langle\boldsymbol{B}(t)\rangle .
$$

Motivated by the LIEB-Robinson bounds, BRAVYi et al. developed an estimation for the spatiotemporal structure of these correlation functions [12]. They made the assumption that for any two local observables $\boldsymbol{O}_{X}$ and $\boldsymbol{O}_{Y}$ the initial correlations decay at least exponentially in space, i.e.,

$$
\left|\left\langle O_{X}(0) O_{Y}(0)\right\rangle_{c}\right| \leq c \exp \left(-\frac{\operatorname{dist}(X, Y)}{\xi}\right)
$$

with some constants $c$ and $\xi$. Then, they showed that the time evolution of the correlation function under a local Hamiltonian is bounded by

$$
\left|\left\langle O_{X}(t) O_{Y}(t)\right\rangle_{c}\right| \leq c^{\prime}(|X|+|Y|) \exp \left(-\frac{\operatorname{dist}(X, Y)-2 v|t|}{\xi^{\prime}}\right),
$$

with different constants $c^{\prime}$ and $\xi^{\prime}$. This bound implies the same light cone effect as the original LiEb-Robinson bounds, only with a changed velocity $2 v$. 

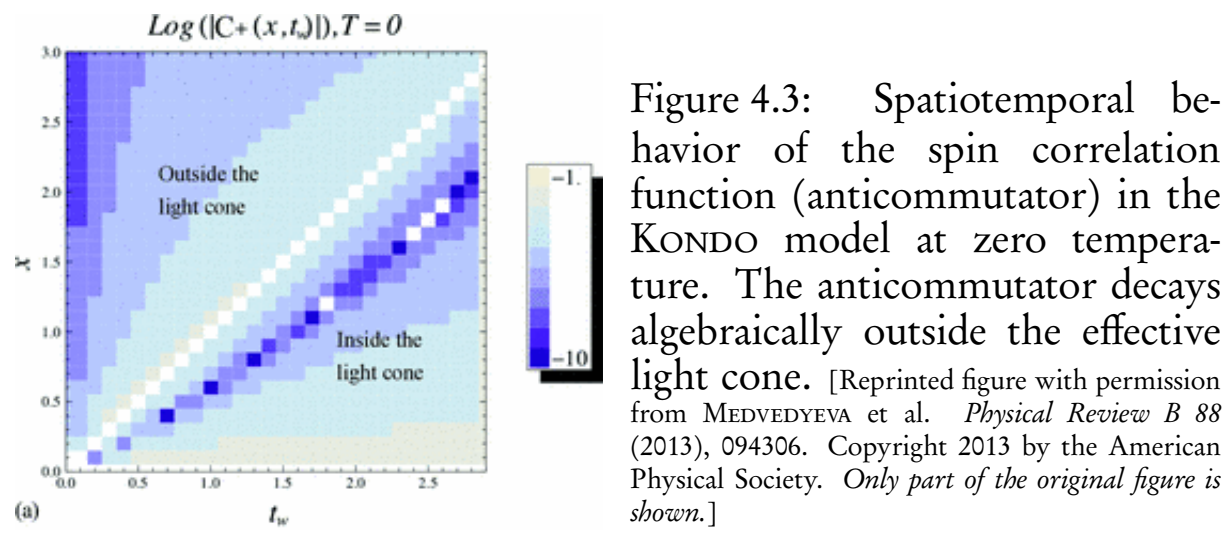

If the initial state is entangled such that the assumption in eq. (4.15) does not hold, this entanglement can enhance the spreading of correlations outside the effective light cone (see Fig. 1.3). A likewise behavior was found in the KonDo model (see Fig. 4.3 for unequal-time spin correlations [80] and in the exactly solvable LUTTINGER model [1].

KASTNER also provided a general bound for equal-time correlation functions for entangled initial states that allows for spreading of long-range correlations that decay algebraically outside the effective light cone [59].

In the following part of this thesis, the boundedness of susceptibilities as well as the dependence of equal-time correlation functions on initial entanglement will be analyzed for the HuBBARD model and accordance with the considerations above will be found. 


\section{Part III}

\section{Calculations and Results}





\section{Chapter 5}

\section{Flow equation analysis of the HubBard model}

In this chapter, we will work out the dynamics of the HUBBARD model in $d>1$ dimensions as defined by eq. (3.10),

$$
H=\sum_{k, \sigma} \epsilon_{k} c_{k \sigma}^{\dagger} c_{k \sigma}+\frac{U}{\Omega} \sum_{k_{1}^{\prime}, k_{1}, k_{2}^{\prime}, k_{2}} c_{k_{1}^{\prime} \uparrow}^{\dagger} c_{k_{1} \uparrow} c_{k_{2}^{\prime} \downarrow}^{\dagger} c_{k_{2} \downarrow} \delta_{k_{1}^{\prime}+k_{2}^{\prime}, k_{1}+k_{2}},
$$

in the framework of unitary perturbation theory, introduced in chapter 2 . The purpose is to study nonequilibrium dynamics in this model, which can be induced by implementing a weak interaction quench. That is, we prepare the system in a thermal state of the noninteracting Hamiltonian and then suddenly switch on the interaction $U$. We will further define this quench protocol in sec. 5.1.

However, for the perturbative solution of the HEISENBERg equation of motion the details of the quench setup do not matter because the time evolution of the observable does not depend on the initial state. Before solving the equation of motion, the first step is to approximately diagonalize the Hamiltonian by means of the coupled flow equations (2.45) and (2.47). As these cannot be solved exactly, all calculations will be carried out perturbatively, where the interaction strength $U$ (or more precisely the ratio between $U$ and some average $\epsilon_{k}$ ) will serve as the expansion parameter. We will first work out the generator of the flow in sec. 5.2 and then solve the flow equations that diagonalize the Hamiltonian in a systematic second-order expansion in sec. 5.3 .

As any dynamical observable of the HUBBARD model depends on the fundamental annihilation and creation operators, we will calculate the real-time evolution of the annihilation operator $c_{k \uparrow}(t)$ by means of the forward-backward scheme that was explained in sec. 2.2.1. For this purpose, we will calculate the unitary flow of $\boldsymbol{c}_{k \uparrow}$ to the energy-diagonal basis in sec. 5.4 and subsequently its time-evolution and backward transformation in sec. 5.5. While some parts of this calculation have already been carried out by MOECKEL and KEHREIN [85], our secondorder expansion of $c_{k \uparrow}(t)$ enables the calculation of all observables that contain products of at most four annihilation and creation operators.

In sec. 5.6, we will show that our perturbative approach is stable up to and including the prethermalization time scale $t \lesssim \rho_{\mathrm{F}}^{-1} U^{-2}$. 


\section{Normal ordering}

In order to have a better control of the vast number of terms that are created throughout the flow equation analysis, we will use normal ordering and hence work with the Hamiltonian

$$
H=\sum_{k, \sigma} \epsilon_{k}: c_{k \sigma}^{\dagger} c_{k \sigma}:+\frac{U}{\Omega} \sum_{k_{1}^{\prime}, k_{1}, k_{2}^{\prime}, k_{2}}: c_{k_{1}^{\prime} \uparrow}^{\dagger} c_{k_{1} \uparrow} c_{k_{2}^{\prime} \downarrow}^{\dagger} c_{k_{2} \downarrow}: \delta_{k_{1}^{\prime}+k_{2}^{\prime}, k_{1}+k_{2}} .
$$

The characters : ... : denote normal ordering due to WICK [116] with respect to the initial state $\left|\psi_{0}\right\rangle$ that the system is prepared in. The main features of normal ordering are explained in App. A, where we also show that normal ordering of the above Hamiltonian does not affect the dynamics.

\subsection{Quantum quench protocol}

A common approach to inducing nonequilibrium dynamics in quantum many-body systems is to implement a quantum quench, which is easier to treat than realistic photoexcitations. The system is prepared in the thermal equilibrium state $\left|\psi_{0}\right\rangle$ of a parameterized Hamiltonian $H(\lambda)$ at $\lambda=\lambda_{0}$. Then, at time $t_{0}$, the value of the Hamiltonian parameter is suddenly changed to $\lambda^{*}$, such that

$$
H(t)= \begin{cases}H\left(\lambda_{0}\right) & \text { for } t<t_{0} \\ H\left(\lambda^{*}\right) & \text { for } t>t_{0}\end{cases}
$$

The time evolution of $\left|\psi_{0}\right\rangle$ for times $t>t_{0}$ is then governed by the Hamiltonian $H\left(\lambda^{*}\right)$. As $\left|\psi_{0}\right\rangle$ is not a thermal state of this quenched Hamiltonian, we will usually observe nonequilibrium behavior.

In this project, we implement an interaction quench in the HubBard Hamiltonian from eq. (5.2). That is, we prepare the system in the thermal state $\left|\psi_{0}\right\rangle$ of the noninteracting Hamiltonian $H_{0}$, hence at $U=0$. This means that

$$
\left\langle\psi_{0}\left|c_{k \sigma}^{\dagger} c_{k \sigma}\right| \psi_{0}\right\rangle=n_{k} \equiv \frac{1}{e^{\beta \epsilon_{k}}+1} \quad(\text { for } \sigma=\uparrow, \downarrow)
$$

is given by the Fermi-Dirac distribution, where $\beta$ is the inverse temperature. We will see that throughout the flow equation analysis all information on the initial state will be encoded in this distribution $n_{k}$.

At time $t=0$, we switch on the interaction to some final value $U$, so that the quench protocol is given by

$$
H(t)= \begin{cases}H_{0} & \text { for } t<0 \\ H_{0}+H_{\text {int }} & \text { for } t \geq 0\end{cases}
$$

Using the HEISENBERg picture, we calculate the time evolution of observables $\boldsymbol{O}(t)$ given by the HEISENBERG equation of motion

$$
\frac{\mathrm{d} O(t)}{\mathrm{d} t}=i[H, O(t)]_{-} .
$$


While the perturbative solution of the HEISENBERG equation of motion in this chapter is independent of the quench protocol and the initial state, the normal ordering will simplify the calculation of expectation values,

$$
\langle O(t)\rangle \equiv\left\langle\psi_{0}|O(t)| \psi_{0}\right\rangle,
$$

if the initial state is the reference state of normal ordering.

\subsection{Canonical generator}

Now, we are looking for the generator of a flow that approximately diagonalizes the Hamiltonian $H=H_{0}+H_{\text {int }}$ for times $t>0$. We use the approach by WEGNER (see sec. 2.3), where our ansatz for the flowing post-quench Hamiltonian up to first order in $U$ is

$$
\begin{aligned}
H(B)= & \underbrace{\sum_{k, \sigma} \epsilon_{k}(B): c_{k \sigma}^{\dagger} c_{k \sigma}}_{H_{0}(B)}:+\underbrace{\frac{1}{\Omega} \sum_{k_{i}^{\prime}, k_{i}} U_{k_{1}^{\prime}, k_{1}, k_{2}^{\prime}, k_{2}}(B): c_{k_{1}^{\prime} \uparrow}^{\dagger} c_{k_{1} \uparrow} c_{k_{2}^{\prime} \downarrow}^{\dagger} c_{k_{2} \downarrow}: \delta_{k_{1}^{\prime}+k_{2}^{\prime}, k_{1}+k_{2}}}_{H_{\text {int }}(B)} \\
& +O\left(U^{2}\right),
\end{aligned}
$$

with the initial conditions $\epsilon_{k}(0)=\epsilon_{k}, U_{k_{1}^{\prime}, k_{1}, k_{2}^{\prime}, k_{2}}(0)=U$. Hence, the canonical generator from eq. (2.47) is given by

$$
\begin{aligned}
\boldsymbol{\eta}(B)= & {\left[H_{0}(B), H_{\mathrm{int}}(B)\right]_{-} } \\
= & \frac{1}{\Omega} \sum_{k} \sum_{k_{i}^{\prime}, k_{i}} \epsilon_{k}(B) U_{k_{1}^{\prime}, k_{1}, k_{2}^{\prime}, k_{2}}(B)\left[: c_{k \uparrow}^{\dagger} c_{k \uparrow}:: c_{k_{1}^{\prime} \uparrow}^{\dagger} c_{k_{1} \uparrow} c_{k_{2}^{\prime} \downarrow}^{\dagger} c_{k_{2} \downarrow}:\right]_{-} \delta_{k_{1}^{\prime}+k_{2}^{\prime}, k_{1}+k_{2}} \\
& +\frac{1}{\Omega} \sum_{k} \sum_{k_{i}^{\prime}, k_{i}} \epsilon_{k}(B) U_{k_{1}^{\prime}, k_{1}, k_{2}^{\prime}, k_{2}}(B)\left[: c_{k_{\downarrow}}^{\dagger} c_{k \downarrow}::: c_{k_{1}^{\prime} \uparrow}^{\dagger} c_{k_{1} \uparrow} c_{k_{2}^{\prime} \downarrow}^{\dagger} c_{k_{2} \downarrow}:\right]_{-} \delta_{k_{1}^{\prime}+k_{2}^{\prime}, k_{1}+k_{2}} \\
& +O\left(U^{2}\right) .
\end{aligned}
$$

The products of normal-ordered expressions in the commutators can be calculated with WICK's second theorem specified in App. A.3. Terms with six normal-ordered annihilation and creation operators commute and cancel out. This is also true for the contractions that result in terms with two normal-ordered operators. Only the contractions that leave terms with four operators do not commute, and we get

$$
\begin{aligned}
\boldsymbol{\eta}(B)= & \frac{1}{\Omega} \sum_{k_{i}^{\prime}, k_{i}} \Delta \epsilon_{k_{1}^{\prime}, k_{1}, k_{2}^{\prime}, k_{2}}(B) U_{k_{1}^{\prime}, k_{1}, k_{2}^{\prime}, k_{2}}(B): c_{k_{1}^{\prime} \uparrow}^{\dagger} c_{k_{1} \uparrow} c_{k_{2}^{\prime} \downarrow}^{\dagger} c_{k_{2} \downarrow}: \delta_{k_{1}^{\prime}+k_{2}^{\prime}, k_{1}+k_{2}} \\
& +O\left(U^{2}\right)
\end{aligned}
$$

with

$$
\Delta \epsilon_{k_{1}^{\prime}, k_{1}, k_{2}^{\prime}, k_{2}}(B) \stackrel{\text { def }}{=} \epsilon_{k_{1}^{\prime}}(B)-\epsilon_{k_{1}}(B)+\epsilon_{k_{2}^{\prime}}(B)-\epsilon_{k_{2}}(B) .
$$

At this point, the coupling of the flow equations becomes clear: The generator of the flow itself has not yet an explicit form but depends on the parameters $\epsilon_{k}(B)$ and $U_{k_{1}^{\prime}, k_{1}, k_{2}^{\prime}, k_{2}}(B)$ from the Hamiltonian, whose flow it generates. Thus, we need to calculate the transformation of the Hamiltonian under the flow, before we are able to explicitly write down the generator of the flow. 


\subsection{Transformation of the Hamiltonian}

The flow equation for the Hamiltonian from eq. (5.8) reads

$$
\frac{\mathrm{d} \boldsymbol{H}(B)}{\mathrm{d} B}=[\boldsymbol{\eta}(B), \boldsymbol{H}(B)]_{-} .
$$

We will first calculate the flow of the Hamiltonian up to first order in $U$, which allows us to write down the canonical generator explicitly. Then, we will turn our attention towards the second-order expansion of the flowing Hamiltonian.

\subsubsection{First-order expansion}

Let us separate eq. (5.12) like

$$
\frac{\mathrm{d} \boldsymbol{H}_{0}(B)}{\mathrm{d} B}+\frac{\mathrm{d} \boldsymbol{H}_{\text {int }}(B)}{\mathrm{d} B}=\left[\boldsymbol{\eta}(B), \boldsymbol{H}_{0}(B)\right]_{-}+\left[\boldsymbol{\eta}(B), \boldsymbol{H}_{\text {int }}(B)\right]_{-} .
$$

We start with calculating the contribution from $\mathrm{H}_{0}$,

$$
\begin{aligned}
{\left[\boldsymbol{\eta}(B), H_{0}(B)\right]_{-}=} & \frac{1}{\Omega} \sum_{k_{i}^{\prime}, k_{i}} \sum_{k, \sigma} \Delta \epsilon_{k_{1}^{\prime}, k_{1}, k_{2}^{\prime}, k_{2}}(B) U_{k_{1}^{\prime}, k_{1}, k_{2}^{\prime}, k_{2}}(B) \epsilon_{k}(B) \\
& \times\left[: c_{k_{1}^{\prime} \uparrow}^{\dagger} c_{k_{1} \uparrow} c_{k_{2}^{\prime} \downarrow}^{\dagger} c_{k_{2} \downarrow}:: c_{k \sigma}^{\dagger} c_{k \sigma}:\right]_{-} \delta_{k_{1}^{\prime}+k_{2}^{\prime}, k_{1}+k_{2}} \\
& +O\left(U^{2}\right) .
\end{aligned}
$$

The calculation of the commutator of the normal-ordered expressions is completely analogous to the calculation from eqs. $(5.9)-(5.10)$, so that

$$
\begin{aligned}
{\left[\boldsymbol{\eta}(B), H_{0}(B)\right]_{-}=} & -\frac{1}{\Omega} \sum_{k_{i}^{\prime}, k_{i}}\left(\Delta \epsilon_{k_{1}^{\prime}, k_{1}, k_{2}^{\prime}, k_{2}}(B)\right)^{2} U_{k_{1}^{\prime}, k_{1}, k_{2}^{\prime}, k_{2}}(B): \boldsymbol{c}_{k_{1}^{\prime} \uparrow}^{\dagger} \boldsymbol{c}_{k_{1} \uparrow} c_{k_{2}^{\prime} \downarrow}^{\dagger} c_{k_{2} \downarrow}: \delta_{k_{1}^{\prime}+k_{2}^{\prime}, k_{1}+k_{2}} \\
& +O\left(U^{2}\right) .
\end{aligned}
$$

The contribution from the interaction part,

$$
\left[\boldsymbol{\eta}(B), H_{\mathrm{int}}(B)\right]_{-}=O\left(U^{2}\right),
$$

is already of second order in $U$.

Now, we compare the operator structures that appear in eq. (5.13). To leading order, the resulting effective flow equations for the Hamiltonian coefficients read

$$
\begin{aligned}
\frac{\mathrm{d} \epsilon_{k}(B)}{\mathrm{d} B}= & 0 \\
& +O\left(U^{2}\right), \\
\frac{\mathrm{d} U_{k_{1}^{\prime}, k_{1}, k_{2}^{\prime}, k_{2}}(B)}{\mathrm{d} B}= & -\left(\Delta \epsilon_{k_{1}^{\prime}, k_{1}, k_{2}^{\prime}, k_{2}}(B)\right)^{2} U_{k_{1}^{\prime}, k_{1}, k_{2}^{\prime}, k_{2}}(B) \\
& +O\left(U^{2}\right) .
\end{aligned}
$$


As by eq. (5.17) $\epsilon_{k}(B)$ is constant, we can easily integrate eq. (5.18), so the solutions to the flow equations are

$$
\begin{aligned}
\epsilon_{k}(B)= & \epsilon_{k} \\
& +O\left(U^{2}\right), \\
U_{k_{1}^{\prime}, k_{1}, k_{2}^{\prime}, k_{2}}(B)= & U e^{-\left(\Delta \epsilon_{k_{1}^{\prime}, k_{1}, k_{2}^{\prime}, k_{2}}\right)^{2} B} \\
& +O\left(U^{2}\right) .
\end{aligned}
$$

Therefore, the flowing Hamiltonian reads

$$
\begin{aligned}
H(B)= & \sum_{k, \sigma} \epsilon_{k}: c_{k \sigma}^{\dagger} c_{k \sigma}:+\frac{U}{\Omega} \sum_{k_{i}^{\prime}, k_{i}} e^{-\left(\Delta \epsilon_{k_{1}^{\prime}, k_{1}, k_{2}^{\prime}, k_{2}}\right)^{2} B}: c_{k_{1}^{\prime} \uparrow}^{\dagger} c_{k_{1} \uparrow} c_{k_{2}^{\prime} \downarrow}^{\dagger} c_{k_{2} \downarrow}: \delta_{k_{1}^{\prime}+k_{2}^{\prime}, k_{1}+k_{2}} \\
& +O\left(U^{2}\right)
\end{aligned}
$$

The exponential function leads to a decay of the collision terms in the interaction part throughout the flow, where highly inelastic terms with a large energy difference $\Delta \epsilon_{k_{1}^{\prime}, k_{1}, k_{2}^{\prime}, k_{2}}$ decay fastest. In the limit $B \rightarrow \infty$, only elastic collision terms with $\Delta \epsilon_{k_{1}^{\prime}, k_{1}, k_{2}^{\prime}, k_{2}}=0$ survive. In the one-dimensional HuBBARD model, where the dispersion relation is linearized [35], the energy difference $\Delta \epsilon_{k_{1}^{\prime}, k_{1}, k_{2}^{\prime}, k_{2}}$ is already zero at $B=0$. This would make the flow featureless, and hence we only consider dimensions $d>1$.

The canonical generator can also be written down explicitly now,

$$
\begin{aligned}
\boldsymbol{\eta}(B)= & \frac{U}{\Omega} \sum_{k_{i}^{\prime}, k_{i}} \Delta \epsilon_{k_{1}^{\prime}, k_{1}, k_{2}^{\prime}, k_{2}} e^{-\left(\Delta \epsilon_{k_{1}^{\prime}, k_{1}, k_{2}^{\prime}, k_{2}}\right)^{2} B}: c_{k_{1}^{\prime} \uparrow}^{\dagger} c_{k_{1} \uparrow} c_{k_{2}^{\prime} \downarrow}^{\dagger} c_{k_{2} \downarrow}: \delta_{k_{1}^{\prime}+k_{2}^{\prime}, k_{1}+k_{2}} \\
& +O\left(U^{2}\right) .
\end{aligned}
$$

In the limit $B \rightarrow \infty$, the generator is completely zero, meaning that $H_{0}$ and $\boldsymbol{H}_{\text {int }}$ will commute in first order.

\subsubsection{Second-order expansion}

The contribution to the flow from eq. (5.16) induces new types of interaction terms in the flowing Hamiltonian that are of second order in $U$, which motivates the ansatz

$$
\begin{aligned}
H(B)= & \sum_{k, \sigma} \epsilon_{k}(B): c_{k \sigma}^{\dagger} c_{k \sigma}:+\frac{1}{\Omega} \sum_{k_{i}^{\prime}, k_{i}} U_{k_{1}^{\prime}, k_{1}, k_{2}^{\prime}, k_{2}}(B): c_{k_{1}^{\prime} \uparrow}^{\dagger} c_{k_{1} \uparrow} c_{k_{2}^{\prime} \downarrow}^{\dagger} c_{k_{2} \downarrow}: \delta_{k_{1}^{\prime}+k_{2}^{\prime}, k_{1}+k_{2}} \\
& +\frac{1}{\Omega} \sum_{k_{i}^{\prime}, k_{i}} \sum_{\sigma} V_{k_{1}^{\prime}, k_{1}, k_{2}^{\prime}, k_{2}}(B): c_{k_{1}^{\prime} \sigma^{\dagger}} c_{k_{1} \sigma} c_{k_{2}^{\prime} \sigma^{\prime}}^{\dagger} c_{k_{2} \sigma}: \delta_{k_{1}^{\prime}+k_{2}^{\prime}, k_{1}+k_{2}} \\
& + \text { higher-order interaction terms } \\
& +O\left(U^{3}\right) .
\end{aligned}
$$

The higher-order interaction terms contain normal-ordered products of at least six annihilation and creation operators and will not contribute to a second-order expansion of twoparticle functions, as we will see later. Hence, we will not consider their explicit flow. 
To second order the canonical generator is given by

$$
\begin{aligned}
\boldsymbol{\eta}(B)= & \frac{1}{\Omega} \sum_{k_{i}^{\prime}, k_{i}} \Delta \epsilon_{k_{1}^{\prime}, k_{1}, k_{2}^{\prime}, k_{2}}(B) U_{k_{1}^{\prime}, k_{1}, k_{2}^{\prime}, k_{2}}(B): c_{k_{1}^{\prime} \uparrow}^{\dagger} c_{k_{1} \uparrow} c_{k_{2}^{\prime} \downarrow}^{\dagger} c_{k_{2} \downarrow}: \delta_{k_{1}^{\prime}+k_{2}^{\prime}, k_{1}+k_{2}} \\
& +\frac{1}{\Omega} \sum_{k_{i}^{\prime}, k_{i}} \sum_{\sigma} \Delta \epsilon_{k_{1}^{\prime}, k_{1}, k_{2}^{\prime}, k_{2}}(B) V_{k_{1}^{\prime}, k_{1}, k_{2}^{\prime}, k_{2}}(B): c_{k_{1}^{\prime} \sigma}^{\dagger} c_{k_{1} \sigma} c_{k_{2}^{\prime} \sigma}^{\dagger} c_{k_{2} \sigma}: \delta_{k_{1}^{\prime}+k_{2}^{\prime}, k_{1}+k_{2}} \\
& + \text { higher-order interaction terms } \\
& +O\left(U^{3}\right) .
\end{aligned}
$$

The flow equation contribution from $H_{0}(B)$ is

$$
\begin{aligned}
{\left[\boldsymbol{\eta}(B), H_{0}(B)\right]_{-}=} & -\frac{1}{\Omega} \sum_{k_{i}^{\prime}, k_{i}}\left(\Delta \epsilon_{k_{1}^{\prime}, k_{1}, k_{2}^{\prime}, k_{2}}\right)^{2} U_{k_{1}^{\prime}, k_{1}, k_{2}^{\prime}, k_{2}}(B): c_{k_{1}^{\prime} \uparrow}^{\dagger} c_{k_{1} \uparrow} c_{k_{2}^{\prime} \downarrow}^{\dagger} c_{k_{2} \downarrow}: \delta_{k_{1}^{\prime}+k_{2}^{\prime}, k_{1}+k_{2}} \\
& -\frac{1}{\Omega} \sum_{k_{i}^{\prime}, k_{i}} \sum_{\sigma}\left(\Delta \epsilon_{k_{1}^{\prime}, k_{1}, k_{2}^{\prime}, k_{2}}\right)^{2} V_{k_{1}^{\prime}, k_{1}, k_{2}^{\prime}, k_{2}}(B): c_{k_{1}^{\prime} \sigma}^{\dagger} c_{k_{1} \sigma} c_{k_{2}^{\prime} \sigma}^{\dagger} c_{k_{2} \sigma}: \delta_{k_{1}^{\prime}+k_{2}^{\prime}, k_{1}+k_{2}} \\
& + \text { higher-order interaction terms } \\
& +O\left(U^{3}\right)
\end{aligned}
$$

while $\boldsymbol{H}_{\text {int }}(B)$ generates the following terms,

$$
\begin{aligned}
{\left[\boldsymbol{\eta}(B), \boldsymbol{H}_{\mathrm{int}}(B)\right]_{-}=} & \frac{2 U^{2}}{\Omega^{2}} \sum_{k, \sigma} \sum_{k_{1}, k_{2}^{\prime}, k_{2}} \Delta \epsilon_{k, k_{1}, k_{2}^{\prime}, k_{2}} e^{-2\left(\Delta \epsilon_{k^{\prime}, k_{1}, k_{2}^{\prime}, k_{2}}\right)^{2} B} \\
& \times\left(\left(1-n_{k_{1}}\right) n_{k_{2}^{\prime}}\left(1-n_{k_{2}}\right)+n_{k_{1}}\left(1-n_{k_{2}^{\prime}}\right) n_{k_{2}}\right): c_{k \sigma}^{\dagger} c_{k \sigma}: \\
& +\frac{U^{2}}{\Omega^{2}} \sum_{k_{i}^{\prime}, k_{i}}\left(\Delta \epsilon_{k_{3}, k_{1}, k_{4}, k_{2}}-\Delta \epsilon_{k_{1}^{\prime}, k_{3}, k_{2}^{\prime}, k_{4}}\right) e^{-\left(\Delta \epsilon_{k_{3}, k_{1}, k_{4}, k_{2}}\right)^{2} B} e^{-\left(\Delta \epsilon_{k_{1}^{\prime}, k_{3}, k_{2}^{\prime}, k_{4}}\right)^{2} B} \\
& \times\left(n_{k_{3}} n_{k_{4}}-\left(1-n_{k_{3}}\right)\left(1-n_{k_{4}}\right)\right): c_{k_{1}^{\prime} \uparrow}^{\dagger} c_{k_{1} \uparrow} c_{k_{2}^{\prime} \downarrow}^{\dagger} c_{k_{2} \downarrow}: \\
& \times \delta_{k_{1}^{\prime}+k_{2}^{\prime}, k_{1}+k_{2}} \delta_{k_{1}^{\prime}+k_{2}^{\prime}, k_{3}+k_{4}} \\
& -\frac{U^{2}}{\Omega^{2}} \sum_{k_{i}^{\prime}, k_{i}}\left(\Delta \epsilon_{k_{3}, k_{1}, k_{2}^{\prime}, k_{4}}-\Delta \epsilon_{k_{1}^{\prime}, k_{3}, k_{4}, k_{2}}\right) e^{-\left(\Delta \epsilon_{k_{3}, k_{1}, k_{2}^{\prime}, k_{4}}\right)^{2} B} e^{-\left(\Delta \epsilon_{k_{1}^{\prime}, k_{3}, k_{4}, k_{2}}\right)^{2} B} \\
& \times\left(n_{k_{3}}-n_{k_{4}}\right): c_{k_{1}^{\prime} \uparrow}^{\dagger} c_{k_{1} \uparrow} c_{k_{2}^{\prime} \downarrow}^{\dagger} c_{k_{2} \downarrow}: \delta_{k_{1}^{\prime}+k_{2}^{\prime}, k_{1}+k_{2}} \delta_{k_{1}^{\prime}+k_{4}, k_{3}+k_{2}} \\
& +\frac{U^{2}}{\Omega^{2}} \sum_{k_{i}^{\prime}, k_{i}} \sum_{\sigma} \Delta \epsilon_{k_{1}^{\prime}, k_{1}, k_{3}^{\prime}, k_{3}} e^{-\left(\Delta \epsilon_{k_{1}^{\prime}, k_{1}, k_{3}^{\prime}, k_{3}}\right)^{2} B} e^{-\left(\Delta \epsilon_{k_{2}^{\prime}, k_{2}, k_{3}, k_{3}^{\prime}}\right)^{2} B}\left(n_{k_{3}^{\prime}}-n_{k_{3}}\right) \\
& \times: c_{k_{1}^{\prime} \sigma}^{\dagger} c_{k_{1} \sigma} c_{k_{2}^{\prime} \sigma}^{\dagger} c_{k_{2} \sigma}: \delta_{k_{1}^{\prime}+k_{2}^{\prime}, k_{1}+k_{2}} \delta_{k_{3}^{\prime}+k_{2}, k_{3}+k_{2}^{\prime}} \\
& + \text { higher-order interaction terms } \\
& +O\left(U^{3}\right) .
\end{aligned}
$$


Here, we have already inserted the first-order results for $\epsilon_{k}(B)$ and $U_{k_{1}^{\prime}, k_{1}, k_{2}^{\prime}, k_{2}}(B)$. The above expressions yield the effective second-order flow equations

$$
\begin{aligned}
& \frac{\mathrm{d} \epsilon_{k}(B)}{\mathrm{d} B}=\frac{2 U^{2}}{\Omega^{2}} \sum_{k_{1}, k_{2}^{\prime}, k_{2}} \Delta \epsilon_{k, k_{1}, k_{2}^{\prime}, k_{2}} e^{-2\left(\Delta \epsilon_{k, k_{1}, k_{2}^{\prime}, k_{2}}\right)^{2} B}\left(\left(1-n_{k_{1}}\right) n_{k_{2}^{\prime}}\left(1-n_{k_{2}}\right)+n_{k_{1}}\left(1-n_{k_{2}^{\prime}}\right) n_{k_{2}}\right) \\
& +O\left(U^{3}\right) \\
& \frac{\mathrm{d} U_{k_{1}^{\prime}, k_{1}, k_{2}^{\prime}, k_{2}}(B)}{\mathrm{d} B}=-\left(\Delta \epsilon_{k_{1}^{\prime}, k_{1}, k_{2}^{\prime}, k_{2}}\right)^{2} U_{k_{1}^{\prime}, k_{1}, k_{2}^{\prime}, k_{2}}(B) \\
& +\frac{U^{2}}{\Omega} \sum_{k_{3}, k_{4}}\left(\Delta \epsilon_{k_{3}, k_{1}, k_{4}, k_{2}}-\Delta \epsilon_{k_{1}^{\prime}, k_{3}, k_{2}^{\prime}, k_{4}}\right) e^{-\left(\Delta \epsilon_{k_{3}, k_{1}, k_{4}, k_{2}}\right)^{2} B} e^{-\left(\Delta \epsilon_{k_{1}^{\prime}, k_{3}, k_{2}^{\prime}, k_{4}}\right)^{2} B} \\
& \times\left(n_{k_{3}} n_{k_{4}}-\left(1-n_{k_{3}}\right)\left(1-n_{k_{4}}\right)\right) \delta_{k_{1}^{\prime}+k_{2}^{\prime}, k_{3}+k_{4}} \\
& -\frac{U^{2}}{\Omega} \sum_{k_{3}, k_{4}}\left(\Delta \epsilon_{k_{3}, k_{1}, k_{2}^{\prime}, k_{4}}-\Delta \epsilon_{k_{1}^{\prime}, k_{3}, k_{4}, k_{2}}\right) e^{-\left(\Delta \epsilon_{k_{3}, k_{1}, k_{2}^{\prime}, k_{4}}\right)^{2} B} e^{-\left(\Delta \epsilon_{k_{1}^{\prime}, k_{3}, k_{4}, k_{2}}\right)^{2} B} \\
& \times\left(n_{k_{3}}-n_{k_{4}}\right) \delta_{k_{1}^{\prime}+k_{4}, k_{3}+k_{2}} \\
& +O\left(U^{3}\right) \text {, } \\
& \frac{\mathrm{d} V_{k_{1}^{\prime}, k_{1}, k_{2}^{\prime}, k_{2}}(B)}{\mathrm{d} B}=-\left(\Delta \epsilon_{k_{1}^{\prime}, k_{1}, k_{2}^{\prime}, k_{2}}\right)^{2} V_{k_{1}^{\prime}, k_{1}, k_{2}^{\prime}, k_{2}}(B) \\
& +\frac{U^{2}}{\Omega} \sum_{k_{3}^{\prime}, k_{3}} \Delta \epsilon_{k_{1}^{\prime}, k_{1}, k_{3}^{\prime}, k_{3}} e^{-\left(\Delta \epsilon_{k_{1}^{\prime}, k_{1}, k_{3}^{\prime}, k_{3}}\right)^{2} B} e^{-\left(\Delta \epsilon_{k_{2}^{\prime}, k_{2}, k_{3}, k_{3}^{\prime}}\right)^{2} B}\left(n_{k_{3}^{\prime}}-n_{k_{3}}\right) \delta_{k_{3}^{\prime}+k_{2}, k_{3}+k_{2}^{\prime}} \\
& +O\left(U^{3}\right) \text {. }
\end{aligned}
$$

Integrating these decoupled equations, we arrive at

$$
\begin{aligned}
\epsilon_{k}(B)= & \epsilon_{k}+\frac{U^{2}}{\Omega^{2}} \sum_{k_{1}, k_{2}^{\prime}, k_{2}} \frac{1-e^{-2\left(\Delta \epsilon_{k, k_{1}, k_{2}^{\prime}, k_{2}}\right)^{2} B}}{\Delta \epsilon_{k, k_{1}, k_{2}^{\prime}, k_{2}}}\left(\left(1-n_{k_{1}}\right) n_{k_{2}^{\prime}}\left(1-n_{k_{2}}\right)+n_{k_{1}}\left(1-n_{k_{2}^{\prime}}\right) n_{k_{2}}\right) \\
& +O\left(U^{3}\right)
\end{aligned}
$$




$$
\begin{aligned}
& U_{k_{1}^{\prime}, k_{1}, k_{2}^{\prime}, k_{2}}(B)=U e^{-\left(\Delta \epsilon_{k_{1}^{\prime}, k_{1}, k_{2}^{\prime}, k_{2}}\right)^{2} B} \\
& +\frac{U^{2}}{\Omega} \sum_{k_{3}, k_{4}}\left(\Delta \epsilon_{k_{3}, k_{1}, k_{4}, k_{2}}-\Delta \epsilon_{k_{1}^{\prime}, k_{3}, k_{2}^{\prime}, k_{4}}\right) \\
& \times \frac{e^{-\left(\Delta \epsilon_{k_{3}, k_{1}, k_{4}, k_{2}}\right)^{2} B} e^{-\left(\Delta \epsilon_{k_{1}^{\prime}, k_{3}, k_{2}^{\prime}, k_{4}}\right)^{2} B}-e^{-\left(\Delta \epsilon_{k_{1}^{\prime}, k_{1}, k_{2}^{\prime}, k_{2}}\right)^{2} B}}{\left(\Delta \epsilon_{k_{1}^{\prime}, k_{1}, k_{2}^{\prime}, k_{2}}\right)^{2}-\left(\Delta \epsilon_{k_{3}, k_{1}, k_{4}, k_{2}}\right)^{2}-\left(\Delta \epsilon_{k_{1}^{\prime}, k_{3}, k_{2}^{\prime}, k_{4}}\right)^{2}} \\
& \times\left(n_{k_{3}} n_{k_{4}}-\left(1-n_{k_{3}}\right)\left(1-n_{k_{4}}\right)\right) \delta_{k_{1}^{\prime}+k_{2}^{\prime}, k_{3}+k_{4}} \\
& -\frac{U^{2}}{\Omega} \sum_{k_{3}, k_{4}}\left(\Delta \epsilon_{k_{3}, k_{1}, k_{2}^{\prime}, k_{4}}-\Delta \epsilon_{k_{1}^{\prime}, k_{3}, k_{4}, k_{2}}\right) \\
& \times \frac{e^{-\left(\Delta \epsilon_{k_{3}, k_{1}, k_{2}^{\prime}, k_{4}}\right)^{2} B} e^{-\left(\Delta \epsilon_{k_{1}^{\prime}, k_{3}, k_{4}, k_{2}}\right)^{2} B}-e^{-\left(\Delta \epsilon_{k_{1}^{\prime}, k_{1}, k_{2}^{\prime}, k_{2}}\right)^{2} B}}{\left(\Delta \epsilon_{k_{1}^{\prime}, k_{1}, k_{2}^{\prime}, k_{2}}\right)^{2}-\left(\Delta \epsilon_{k_{3}, k_{1}, k_{2}^{\prime}, k_{4}}\right)^{2}-\left(\Delta \epsilon_{k_{1}^{\prime}, k_{3}, k_{4}, k_{2}}\right)^{2}} \\
& \times\left(n_{k_{3}}-n_{k_{4}}\right) \delta_{k_{1}^{\prime}+k_{4}, k_{3}+k_{2}} \\
& +O\left(U^{3}\right) \\
& V_{k_{1}^{\prime}, k_{1}, k_{2}^{\prime}, k_{2}}(B)=-\frac{U^{2}}{\Omega} \sum_{k_{3}^{\prime}, k_{3}} \Delta \epsilon_{k_{1}^{\prime}, k_{1}, k_{3}^{\prime}, k_{3}} \frac{e^{-\left(\Delta \epsilon_{k_{1}^{\prime}, k_{1}, k_{3}^{\prime}, k_{3}}\right)^{2} B} e^{-\left(\Delta \epsilon_{k_{2}^{\prime}, k_{2}, k_{3}, k_{3}^{\prime}}\right)^{2} B}-e^{-\left(\Delta \epsilon_{k_{1}^{\prime}, k_{1}, k_{2}^{\prime}, k_{2}}\right)^{2} B}}{\left(\Delta \epsilon_{k_{1}^{\prime}, k_{1}, k_{3}^{\prime}, k_{3}}\right)^{2}+\left(\Delta \epsilon_{k_{2}^{\prime}, k_{2}, k_{3}, k_{3}^{\prime}}\right)^{2}-\left(\Delta \epsilon_{k_{1}^{\prime}, k_{1}, k_{2}^{\prime}, k_{2}}\right)^{2}} \\
& \times\left(n_{k_{3}^{\prime}}-n_{k_{3}}\right) \delta_{k_{3}^{\prime}+k_{2}, k_{3}+k_{2}^{\prime}} \\
& +O\left(U^{3}\right) \text {. }
\end{aligned}
$$

\subsubsection{Energy-diagonal Hamiltonian}

We have applied the flow equations in order to diagonalize the Hamiltonian. From eqs. 5.30) - (5.32), we can read off the following second-order structure of the Hamiltonian at $B=\infty$,

$$
\begin{aligned}
\tilde{H}= & \sum_{k, \sigma} \tilde{\epsilon}_{k}: c_{k \sigma}^{\dagger} c_{k \sigma}:+\frac{U}{\Omega} \sum_{k_{i}^{\prime}, k_{i}}: c_{k_{1}^{\prime} \uparrow}^{\dagger} c_{k_{1} \uparrow} c_{k_{2}^{\prime} \downarrow}^{\dagger} c_{k_{2} \downarrow}: \delta_{\epsilon_{k_{1}^{\prime}}+\epsilon_{k_{2}^{\prime}}, \epsilon_{k_{1}}+\epsilon_{k_{2}}} \delta_{k_{1}^{\prime}+k_{2}^{\prime}, k_{1}+k_{2}} \\
& + \text { higher-order interaction terms } \\
& +O\left(U^{3}\right)
\end{aligned}
$$

with a renormalized one-particle energy

$$
\tilde{\epsilon}_{k}=\epsilon_{k}+\frac{U^{2}}{\Omega^{2}} \sum_{k_{1}, k_{2}^{\prime}, k_{2}} \frac{1}{\Delta \epsilon_{k, k_{1}, k_{2}^{\prime}, k_{2}}}\left(\left(1-n_{k_{1}}\right) n_{k_{2}^{\prime}}\left(1-n_{k_{2}}\right)+n_{k_{1}}\left(1-n_{k_{2}^{\prime}}\right) n_{k_{2}}\right)+O\left(U^{3}\right) .
$$

We realize that the interaction part of the Hamiltonian greatly simplifies throughout the flow. While the second-order corrections to $U_{k_{1}^{\prime}, k_{1}, k_{2}^{\prime}, k_{2}}(B)$ and $V_{k_{1}^{\prime}, k_{1}, k_{2}^{\prime}, k_{2}}(B)$ flow to zero, only the energy-diagonal part of the original interaction that resembles elastic collision processes (with $\left.\epsilon_{k_{1}}+\epsilon_{k_{2}}=\epsilon_{k_{1}^{\prime}}+\epsilon_{k_{2}^{\prime}}\right)$ remains. However, we cannot get rid of this remaining term, which still induces secular terms, when calculating the time evolution of observables. 
The elastic collision term is exactly the one that appears in the quantum BoltzmanN equation that describes the thermalization dynamics and only becomes relevant at a time scale $t \sim \rho_{\mathrm{F}}^{-3} U^{-4}[85,98]$. In sec. 5.6, we will see that our perturbative approach is only stable up to and including times $t \lesssim \rho_{\mathrm{F}}^{-1} U^{-2}$ and hence we omit these elastic contributions.

The higher-order interaction terms in eq. (5.33) will not influence second-order expansions of two-particle functions anyway.

The energy-diagonal dispersion relation from eq. (5.34) has a second-order shift. For observables $O(t)=O^{(0)}+U O^{(1)}(t)+O\left(U^{2}\right)$, whose time-dependence only appears in the first- or higher-order correction, the energy-shift does not affect the second-order expansion of $\boldsymbol{O}(t)$. Hence, at $B=\infty$, the time evolution is only due to $\tilde{H}_{0}=\sum_{k, \sigma} \epsilon_{k}: c_{k \sigma}^{\dagger} c_{k \sigma}:$. This Hamiltonian may seem trivial, so let us mention again that the complex dynamics of the HubBard model is encoded in the generator from eq. (5.24) that affects the flow of the observables and therefore indirectly also their dynamics.

In the next section, we will see how an observable, more precisely the annihilation operator, transforms under this flow.

\subsection{Transformation of the annihilation operator}

According to eq. (2.48) the unitarily transformed annihilation operator is subject to the flow equation

$$
\frac{\mathrm{d} \boldsymbol{c}_{k \uparrow}(B)}{\mathrm{d} B}=\left[\boldsymbol{\eta}(B), c_{k \uparrow}(B)\right]_{-} .
$$

We realize that the canonical generator from eq. (5.24) generates new types of interaction terms, which motivates the following ansatz for the flowing annihilation operator,

$$
\begin{aligned}
c_{k \uparrow}(B)= & h_{k}(B): c_{k \uparrow}: \\
& +\sum_{k_{i}^{\prime}, k_{i}} F_{k, k_{1}, k_{2}^{\prime}, k_{2}}(B): c_{k_{1} \uparrow} c_{k_{2}^{\prime} \downarrow}^{\dagger} c_{k_{2} \downarrow}: \delta_{k+k_{2}^{\prime}, k_{1}+k_{2}} \\
& +\sum_{k_{i}^{\prime}, k_{i}} G_{k, k_{1}, k_{2}^{\prime}, k_{2}}(B): c_{k_{1} \uparrow} c_{k_{2}^{\prime} \uparrow}^{\dagger} c_{k_{2} \uparrow}: \delta_{k+k_{2}, k_{1}+k_{2}} \\
& +\sum_{k_{i}^{\prime}, k_{i}} R_{k, k_{1}, k_{2}^{\prime}, k_{2}, k_{3}^{\prime}, k_{3}}(B): c_{k_{1} \uparrow} c_{k_{2}^{\prime} \downarrow}^{\dagger} c_{k_{2} \downarrow} c_{k_{3}^{\prime} \uparrow}^{\dagger} c_{k_{3} \uparrow}: \delta_{k+k_{2}^{\prime}+k_{3}^{\prime}, k_{1}+k_{2}+k_{3}} \\
& +\sum_{k_{i}^{\prime}, k_{i}} S_{k, k_{1}, k_{2}^{\prime}, k_{2}, k_{3}^{\prime}, k_{3}}(B): c_{k_{1} \uparrow} c_{k_{2}^{\prime} \downarrow}^{\dagger} c_{k_{2} \downarrow} c_{k_{3}^{\prime} \downarrow}^{\dagger} c_{k_{3} \downarrow}: \delta_{k+k_{2}^{\prime}+k_{3}^{\prime}, k_{1}+k_{2}+k_{3}} \\
& +O\left(U^{3}\right),
\end{aligned}
$$

where initially $h_{k}(B=0)=1$ and all the other coefficients are zero at $B=0$. We analyze that

$$
\begin{aligned}
h_{k}(B) & =O(1), \\
F_{k, k_{1}, k_{2}^{\prime}, k_{2}}(B) & =O(U), \\
G_{k, k_{1}, k_{2}^{\prime}, k_{2}}(B) & =O\left(U^{2}\right), \\
R_{k, k_{1}, k_{2}^{\prime}, k_{2}, k_{3}^{\prime}, k_{3}}(B) & =O\left(U^{2}\right), \\
S_{k, k_{1}, k_{2}^{\prime}, k_{2}, k_{3}^{\prime}, k_{3}}(B) & =O\left(U^{2}\right) .
\end{aligned}
$$


The coefficients $R_{k, k_{1}, k_{2}^{\prime}, k_{2}, k_{3}^{\prime}, k_{3}}(B)$ and $S_{k, k_{1}, k_{2}^{\prime}, k_{2}, k_{3}^{\prime}, k_{3}}(B)$ do not contribute to a second-order expansion of two-particle functions. Hence, we will not consider their flow, which would depend on the higher-order interaction terms in eq. (5.24).

Now, we insert the ansatz for the annihilation operator from eq. (5.36) into the flow equation 5.35). Comparing both sides of the equation results in the following effective flow equations for the coefficients,

$$
\begin{aligned}
& \frac{\mathrm{d} h_{k}(B)}{\mathrm{d} B}=\frac{U}{\Omega} \sum_{k_{1}, k_{2}^{\prime}, k_{2}} \Delta \epsilon_{k, k_{1}, k_{2}^{\prime}, k_{2}} e^{-\left(\Delta \epsilon_{k, k_{1}, k_{2}^{\prime}, k_{2}}\right)^{2} B} F_{k, k_{1}, k_{2}^{\prime}, k_{2}}(B) \\
& \times\left(\left(1-n_{k_{1}}\right) n_{k_{2}^{\prime}}\left(1-n_{k_{2}}\right)+n_{k_{1}}\left(1-n_{k_{2}^{\prime}}\right) n_{k_{2}}\right) \delta_{k+k_{2}^{\prime}, k_{1}+k_{2}} \\
& +O\left(U^{3}\right) \\
& \frac{\mathrm{d} F_{k, k_{1}, k_{2}^{\prime}, k_{2}}(B)}{\mathrm{d} B}=-\frac{U}{\Omega} \Delta \epsilon_{k, k_{1}, k_{2}^{\prime}, k_{2}} e^{-\left(\Delta \epsilon_{k, k_{1}, k_{2}^{\prime}, k_{2}}\right)^{2} B} h_{k}(B) \\
& -\frac{U^{2}}{\Omega^{2}} \sum_{k_{3}, k_{4}} \Delta \epsilon_{k, k_{1}, k_{2}^{\prime}, k_{2}}\left(\Delta \epsilon_{k_{3}, k_{1}, k_{4}, k_{2}}-\Delta \epsilon_{k, k_{3}, k_{2}^{\prime}, k_{4}}\right) h_{k}(B) \\
& \times \frac{e^{-\left(\Delta \epsilon_{k_{3}, k_{1}, k_{4}, k_{2}}\right)^{2} B} e^{-\left(\Delta \epsilon_{k, k_{3}, k_{2}^{\prime}, k_{4}}\right)^{2} B}-e^{-\left(\Delta \epsilon_{k, k_{1}, k_{2}^{\prime}, k_{2}}\right)^{2} B}}{\left(\Delta \epsilon_{k, k_{1}, k_{2}^{\prime}, k_{2}}\right)^{2}-\left(\Delta \epsilon_{k_{3}, k_{1}, k_{4}, k_{2}}\right)^{2}-\left(\Delta \epsilon_{k, k_{3}, k_{2}^{\prime}, k_{4}}\right)^{2}} \\
& \times\left(n_{k_{3}} n_{k_{4}}-\left(1-n_{k_{3}}\right)\left(1-n_{k_{4}}\right)\right) \delta_{k+k_{2}^{\prime}, k_{3}+k_{4}} \\
& +\frac{U^{2}}{\Omega^{2}} \sum_{k_{3}, k_{4}} \Delta \epsilon_{k, k_{1}, k_{2}^{\prime}, k_{2}}\left(\Delta \epsilon_{k_{3}, k_{1}, k_{2}^{\prime}, k_{4}}-\Delta \epsilon_{k, k_{3}, k_{4}, k_{2}}\right) h_{k}(B) \\
& \times \frac{e^{-\left(\Delta \epsilon_{k_{3}, k_{1}, k_{2}^{\prime}, k_{4}}\right)^{2} B} e^{-\left(\Delta \epsilon_{k, k_{3}, k_{4}, k_{2}}\right)^{2} B}-e^{-\left(\Delta \epsilon_{k, k_{1}, k_{2}^{\prime}, k_{2}}\right)^{2} B}}{\left(\Delta \epsilon_{k, k_{1}, k_{2}^{\prime}, k_{2}}\right)^{2}-\left(\Delta \epsilon_{k_{3}, k_{1}, k_{2}^{\prime}, k_{4}}\right)^{2}-\left(\Delta \epsilon_{k, k_{3}, k_{4}, k_{2}}\right)^{2}} \\
& \times\left(n_{k_{3}}-n_{k_{4}}\right) \delta_{k+k_{4}, k_{3}+k_{2}} \\
& -\frac{U}{\Omega} \sum_{k_{3}^{\prime}, k_{3}} \Delta \epsilon_{k_{3}^{\prime}, k_{1}, k_{2}^{\prime}, k_{3}} e^{-\left(\Delta \epsilon_{k_{3}^{\prime}, k_{1}, k_{2}^{\prime}, k_{3}}\right)^{2} B} F_{k, k_{3}^{\prime}, k_{3}, k_{2}}(B) \\
& \times\left(n_{k_{3}^{\prime}}-n_{k_{3}}\right) \delta_{k+k_{3}, k_{3}^{\prime}+k_{2}} \\
& -\frac{U}{\Omega} \sum_{k_{3}^{\prime}, k_{3}} \Delta \epsilon_{k_{3}, k_{1}, k_{3}^{\prime}, k_{2}} e^{-\left(\Delta \epsilon_{k_{3}, k_{1}, k_{3}^{\prime}, k_{2}}\right)^{2} B} F_{k, k_{3}, k_{2}^{\prime}, k_{3}^{\prime}}(B) \\
& \times\left(\left(1-n_{k_{3}^{\prime}}\right)\left(1-n_{k_{3}}\right)-n_{k_{3}^{\prime}} n_{k_{3}}\right) \delta_{k+k_{2}^{\prime}, k_{3}+k_{3}^{\prime}} \\
& +O\left(U^{3}\right) \text {, }
\end{aligned}
$$




$$
\begin{aligned}
\frac{\mathrm{d} G_{k, k_{1}, k_{2}^{\prime}, k_{2}}(B)}{\mathrm{d} B}=\frac{U}{\Omega} \sum_{k_{3}^{\prime}, k_{3}} \Delta \epsilon_{k_{3}^{\prime}, k_{3}, k_{2}^{\prime}, k_{2}} e^{-\left(\Delta \epsilon_{k_{3}^{\prime}, k_{3}, k_{2}^{\prime}, k_{2}}\right)^{2} B} F_{k, k_{1}, k_{3}, k_{3}^{\prime}}(B) \\
\quad \times\left(n_{k_{3}^{\prime}}-n_{k_{3}}\right) \delta_{k_{2}^{\prime}+k_{3}^{\prime}, k_{2}+k_{3}} \\
+\frac{U^{2}}{\Omega^{2}} \sum_{k_{3}^{\prime}, k_{3}}\left(\Delta \epsilon_{k, k_{1}, k_{2}^{\prime}, k_{2}}\right)\left(\Delta \epsilon_{k, k_{1}, k_{3}^{\prime}, k_{3}}-\Delta \epsilon_{k_{2}^{\prime}, k_{2}, k_{3}, k_{3}^{\prime}}\right) h_{k}(B) \\
\quad \times \frac{e^{-\left(\Delta \epsilon_{k, k_{1}, k_{3}^{\prime}, k_{3}}\right)^{2} B} e^{-\left(\Delta \epsilon_{k_{2}^{\prime}, k_{2}, k_{3}, k_{3}^{\prime}}\right)^{2} B}-e^{-\left(\Delta \epsilon_{k, k_{1}, k_{2}^{\prime}, k_{2}}\right)^{2} B}}{\left(\Delta \epsilon_{k, k_{1}, k_{3}^{\prime}, k_{3}}\right)^{2}+\left(\Delta \epsilon_{k_{2}^{\prime}, k_{2}, k_{3}, k_{3}^{\prime}}\right)^{2}-\left(\Delta \epsilon_{k, k_{1}, k_{2}^{\prime}, k_{2}}\right)^{2}} \\
\quad \times\left(n_{k_{3}^{\prime}}-n_{k_{3}}\right) \delta_{k_{3}^{\prime}+k_{2}, k_{3}+k_{2}^{\prime}} \\
+
\end{aligned}
$$

In order to solve these equations perturbatively, we start with eq. (5.42), where we can read off that $h_{k}(B)=1+O\left(U^{2}\right)$. Using this zeroth-order result for the first-order part of eq. (5.43), we can directly integrate and get

$$
\begin{aligned}
F_{k, k_{1}, k_{2}^{\prime}, k_{2}}(B)= & -\frac{U}{\Omega} \frac{1-e^{-\left(\Delta \epsilon_{k, k_{1}, k_{2}^{\prime}, k_{2}}\right)^{2} B}}{\Delta \epsilon_{k, k_{1}, k_{2}^{\prime}, k_{2}}} \\
& +O\left(U^{2}\right) .
\end{aligned}
$$

This first-order expression for $F_{k, k_{1}, k_{2}^{\prime}, k_{2}}(B)$ can now be used for a second-order solution of eqs. (5.42) - (5.44). A simple integration results in

$$
\begin{aligned}
h_{k}(B)= & 1-\frac{U^{2}}{2 \Omega^{2}} \sum_{k_{1}, k_{2}^{\prime}, k_{2}}\left(\frac{1-e^{-\left(\Delta \epsilon_{k, k_{1}, k_{2}^{\prime}, k_{2}}\right)^{2} B}}{\Delta \epsilon_{k, k_{1}, k_{2}^{\prime}, k_{2}}}\right)^{2} \\
& \times\left(\left(1-n_{k_{1}}\right) n_{k_{2}^{\prime}}\left(1-n_{k_{2}}\right)+n_{k_{1}}\left(1-n_{k_{2}^{\prime}}\right) n_{k_{2}}\right) \delta_{k+k_{2}^{\prime}, k_{1}+k_{2}} \\
& +O\left(U^{3}\right),
\end{aligned}
$$




$$
\begin{aligned}
& F_{k, k_{1}, k_{2}^{\prime}, k_{2}}(B)=-\frac{U}{\Omega} \frac{1-e^{-\left(\Delta \epsilon_{k, k_{1}, k_{2}^{\prime}, k_{2}}\right)^{2} B}}{\Delta \epsilon_{k, k_{1}, k_{2}^{\prime}, k_{2}}} \\
& -\frac{U^{2}}{\Omega^{2}} \sum_{k_{3}, k_{4}} \frac{\Delta \epsilon_{k, k_{1}, k_{2}^{\prime}, k_{2}}\left(\Delta \epsilon_{k_{3}, k_{1}, k_{4}, k_{2}}-\Delta \epsilon_{k, k_{3}, k_{2}^{\prime}, k_{4}}\right)}{\left(\Delta \epsilon_{k, k_{1}, k_{2}^{\prime}, k_{2}}\right)^{2}-\left(\Delta \epsilon_{k_{3}, k_{1}, k_{4}, k_{2}}\right)^{2}-\left(\Delta \epsilon_{k, k_{3}, k_{2}^{\prime}, k_{4}}\right)^{2}} \\
& \times\left(\frac{1-e^{-\left(\Delta \epsilon_{k_{3}, k_{1}, k_{4}, k_{2}}\right)^{2} B} e^{-\left(\Delta \epsilon_{k, k_{3}, k_{2}^{\prime}, k_{4}}\right)^{2} B}}{\left(\Delta \epsilon_{k_{3}, k_{1}, k_{4}, k_{2}}\right)^{2}+\left(\Delta \epsilon_{k, k_{3}, k_{2}^{\prime}, k_{4}}\right)^{2}}-\frac{1-e^{-\left(\Delta \epsilon_{k, k_{1}, k_{2}^{\prime}, k_{2}}\right)^{2} B}}{\left(\Delta \epsilon_{k, k_{1}, k_{2}^{\prime}, k_{2}}\right)^{2}}\right) \\
& \times\left(n_{k_{3}} n_{k_{4}}-\left(1-n_{k_{3}}\right)\left(1-n_{k_{4}}\right)\right) \delta_{k+k_{2}^{\prime}, k_{3}+k_{4}} \\
& +\frac{U^{2}}{\Omega^{2}} \sum_{k_{3}, k_{4}} \frac{\Delta \epsilon_{k, k_{1}, k_{2}^{\prime}, k_{2}}\left(\Delta \epsilon_{k_{3}, k_{1}, k_{2}^{\prime}, k_{4}}-\Delta \epsilon_{k, k_{3}, k_{4}, k_{2}}\right)}{\left(\Delta \epsilon_{k, k_{1}, k_{2}^{\prime}, k_{2}}\right)^{2}-\left(\Delta \epsilon_{k_{3}, k_{1}, k_{2}^{\prime}, k_{4}}\right)^{2}-\left(\Delta \epsilon_{k, k_{3}, k_{4}, k_{2}}\right)^{2}} \\
& \times\left(\frac{1-e^{-\left(\Delta \epsilon_{k_{3}, k_{1}, k_{2}^{\prime}, k_{4}}\right)^{2} B} e^{-\left(\Delta \epsilon_{k, k_{3}, k_{4}, k_{2}}\right)^{2} B}}{\left(\Delta \epsilon_{k_{3}, k_{1}, k_{2}^{\prime}, k_{4}}\right)^{2}+\left(\Delta \epsilon_{k, k_{3}, k_{4}, k_{2}}\right)^{2}}-\frac{1-e^{-\left(\Delta \epsilon_{k, k_{1}, k_{2}^{\prime}, k_{2}}\right)^{2} B}}{\left(\Delta \epsilon_{k, k_{1}, k_{2}^{\prime}, k_{2}}\right)^{2}}\right) \\
& \times\left(n_{k_{3}}-n_{k_{4}}\right) \delta_{k+k_{4}, k_{3}+k_{2}} \\
& +\frac{U^{2}}{\Omega^{2}} \sum_{k_{3}^{\prime}, k_{3}} \frac{\Delta \epsilon_{k_{3}^{\prime}, k_{1}, k_{2}^{\prime}, k_{3}}}{\Delta \epsilon_{k, k_{3}^{\prime}, k_{3}, k_{2}}}\left(\frac{1-e^{-\left(\Delta \epsilon_{k_{3}^{\prime}, k_{1}, k_{2}^{\prime}, k_{3}}\right)^{2} B}}{\left(\Delta \epsilon_{k_{3}^{\prime}, k_{1}, k_{2}^{\prime}, k_{3}}\right)}-\frac{1-e^{-\left(\Delta \epsilon_{k_{3}^{\prime}, k_{1}, k_{2}^{\prime}, k_{3}}\right)^{2} B} e^{-\left(\Delta \epsilon_{k, k_{3}^{\prime}, k_{3}, k_{2}}\right)^{2} B}}{\left(\Delta \epsilon_{k_{3}^{\prime}, k_{1}, k_{2}^{\prime}, k_{3}}\right)^{2}+\left(\Delta \epsilon_{k, k_{3}^{\prime}, k_{3}, k_{2}}\right)^{2}}\right) \\
& \times\left(n_{k_{3}^{\prime}}-n_{k_{3}}\right) \delta_{k+k_{3}, k_{3}^{\prime}+k_{2}} \\
& +\frac{U^{2}}{\Omega^{2}} \sum_{k_{3}^{\prime}, k_{3}} \frac{\Delta \epsilon_{k_{3}, k_{1}, k_{3}^{\prime}, k_{2}}}{\Delta \epsilon_{k, k_{3}, k_{2}^{\prime}, k_{3}^{\prime}}}\left(\frac{1-e^{-\left(\Delta \epsilon_{k_{3}, k_{1}, k_{3}^{\prime}, k_{2}}\right)^{2} B}}{\left(\Delta \epsilon_{k_{3}, k_{1}, k_{3}^{\prime}, k_{2}}\right)^{2}}-\frac{1-e^{-\left(\Delta \epsilon_{k_{3}, k_{1}, k_{3}^{\prime}, k_{2}}\right)^{2} B} e^{-\left(\Delta \epsilon_{k, k_{3}, k_{2}^{\prime}, k_{3}^{\prime}}\right)^{2} B}}{\left(\Delta \epsilon_{k_{3}, k_{1}, k_{3}, k_{2}}\right)^{2}+\left(\Delta \epsilon_{k, k_{3}, k_{2}^{\prime}, k_{3}^{\prime}}\right)^{2}}\right) \\
& \times\left(\left(1-n_{k_{3}^{\prime}}\right)\left(1-n_{k_{3}}\right)-n_{k_{3}^{\prime}} n_{k_{3}}\right) \delta_{k+k_{2}^{\prime}, k_{3}+k_{3}^{\prime}} \\
& +O\left(U^{3}\right) \text {, } \\
& G_{k, k_{1}, k_{2}^{\prime}, k_{2}}(B)=\frac{U^{2}}{\Omega^{2}} \sum_{k_{3}^{\prime}, k_{3}} \frac{\Delta \epsilon_{k_{3}^{\prime}, k_{3}, k_{2}^{\prime}, k_{2}}}{\Delta \epsilon_{k, k_{1}, k_{3}, k_{3}^{\prime}}}\left(\frac{1-e^{-\left(\Delta \epsilon_{k_{3}^{\prime}, k_{3}, k_{2}^{\prime}, k_{2}}\right)^{2} B} e^{-\left(\Delta \epsilon_{k, k_{1}, k_{3}, k_{3}^{\prime}}\right)^{2} B}}{\left(\Delta \epsilon_{k_{3}^{\prime}, k_{3}, k_{2}^{\prime}, k_{2}}\right)^{2}+\left(\Delta \epsilon_{k, k_{1}, k_{3}, k_{3}^{\prime}}\right)^{2}}-\frac{1-e^{-\left(\Delta \epsilon_{k_{3}^{\prime}, k_{3}, k_{2}^{\prime}, k_{2}}\right)^{2} B}}{\left(\Delta \epsilon_{k_{3}^{\prime}, k_{3}, k_{2}^{\prime}, k_{2}}\right)^{2}}\right) \\
& \times\left(n_{k_{3}^{\prime}}-n_{k_{3}}\right) \delta_{k_{2}^{\prime}+k_{3}^{\prime}, k_{2}+k_{3}} \\
& +\frac{U^{2}}{\Omega^{2}} \sum_{k_{3}^{\prime}, k_{3}} \frac{\left(\Delta \epsilon_{k, k_{1}, k_{2}^{\prime}, k_{2}}\right)\left(\Delta \epsilon_{k, k_{1}, k_{3}^{\prime}, k_{3}}-\Delta \epsilon_{k_{2}^{\prime}, k_{2}, k_{3}, k_{3}^{\prime}}\right)}{\left(\Delta \epsilon_{k, k_{1}, k_{3}^{\prime}, k_{3}}\right)^{2}+\left(\Delta \epsilon_{k_{2}^{\prime}, k_{2}, k_{3}, k_{3}^{\prime}}\right)^{2}-\left(\Delta \epsilon_{k, k_{1}, k_{2}^{\prime}, k_{2}}\right)^{2}}
\end{aligned}
$$

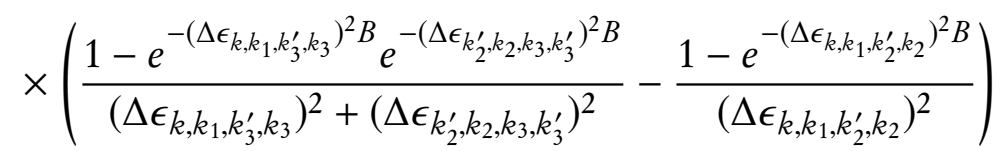

$$
\begin{aligned}
& \times\left(n_{k_{3}^{\prime}}-n_{k_{3}}\right) \delta_{k_{3}^{\prime}+k_{2}, k_{3}+k_{2}^{\prime}} \\
& +O\left(U^{3}\right) \text {. }
\end{aligned}
$$


In the limit $B \rightarrow \infty$, where the Hamiltonian becomes energy-diagonal, the coefficients of the flowing annihilation operator are

$$
\begin{aligned}
& \tilde{h}_{k}=1-\frac{U^{2}}{2 \Omega^{2}} \sum_{k_{1}, k_{2}^{\prime}, k_{2}} \frac{1}{\left(\Delta \epsilon_{k, k_{1}, k_{2}^{\prime}, k_{2}}\right)^{2}}\left(\left(1-n_{k_{1}}\right) n_{k_{2}^{\prime}}\left(1-n_{k_{2}}\right)+n_{k_{1}}\left(1-n_{k_{2}^{\prime}}\right) n_{k_{2}}\right) \delta_{k+k_{2}^{\prime}, k_{1}+k_{2}} \\
& +O\left(U^{3}\right) \text {, } \\
& \tilde{F}_{k, k_{1}, k_{2}^{\prime}, k_{2}}=-\frac{U}{\Omega} \frac{1}{\Delta \epsilon_{k, k_{1}, k_{2}^{\prime}, k_{2}}} \\
& -\frac{U^{2}}{\Omega^{2}} \sum_{k_{3}, k_{4}} \frac{\Delta \epsilon_{k, k_{1}, k_{2}^{\prime}, k_{2}}\left(\Delta \epsilon_{k_{3}, k_{1}, k_{4}, k_{2}}-\Delta \epsilon_{k, k_{3}, k_{2}^{\prime}, k_{4}}\right)}{\left(\Delta \epsilon_{k, k_{1}, k_{2}^{\prime}, k_{2}}\right)^{2}-\left(\Delta \epsilon_{k_{3}, k_{1}, k_{4}, k_{2}}\right)^{2}-\left(\Delta \epsilon_{k, k_{3}, k_{2}, k_{4}}\right)^{2}} \\
& \times\left(\frac{1}{\left(\Delta \epsilon_{k_{3}, k_{1}, k_{4}, k_{2}}\right)^{2}+\left(\Delta \epsilon_{k, k_{3}, k_{2}^{\prime}, k_{4}}\right)^{2}}-\frac{1}{\left(\Delta \epsilon_{k, k_{1}, k_{2}^{\prime}, k_{2}}\right)^{2}}\right) \\
& \times\left(n_{k_{3}} n_{k_{4}}-\left(1-n_{k_{3}}\right)\left(1-n_{k_{4}}\right)\right) \delta_{k+k_{2}^{\prime}, k_{3}+k_{4}} \\
& +\frac{U^{2}}{\Omega^{2}} \sum_{k_{3}, k_{4}} \frac{\Delta \epsilon_{k, k_{1}, k_{2}^{\prime}, k_{2}}\left(\Delta \epsilon_{k_{3}, k_{1}, k_{2}^{\prime}, k_{4}}-\Delta \epsilon_{k, k_{3}, k_{4}, k_{2}}\right)}{\left(\Delta \epsilon_{k, k_{1}, k_{2}^{\prime}, k_{2}}\right)^{2}-\left(\Delta \epsilon_{k_{3}, k_{1}, k_{2}^{\prime}, k_{4}}\right)^{2}-\left(\Delta \epsilon_{k, k_{3}, k_{4}, k_{2}}\right)^{2}} \\
& \times\left(\frac{1}{\left(\Delta \epsilon_{k_{3}, k_{1}, k_{2}^{\prime}, k_{4}}\right)^{2}+\left(\Delta \epsilon_{k, k_{3}, k_{4}, k_{2}}\right)^{2}}-\frac{1}{\left(\Delta \epsilon_{k, k_{1}, k_{2}^{2}, k_{2}}\right)^{2}}\right) \\
& \times\left(n_{k_{3}}-n_{k_{4}}\right) \delta_{k+k_{4}, k_{3}+k_{2}} \\
& +\frac{U^{2}}{\Omega^{2}} \sum_{k_{3}^{\prime}, k_{3}} \frac{\Delta \epsilon_{k_{3}^{\prime}, k_{1}, k_{2}^{\prime}, k_{3}}}{\Delta \epsilon_{k, k_{3}^{\prime}, k_{3}, k_{2}}}\left(\frac{1}{\left(\Delta \epsilon_{k_{3}^{\prime}, k_{1}, k_{2}^{\prime}, k_{3}}\right)}-\frac{1}{\left(\Delta \epsilon_{k_{3}^{\prime}, k_{1}, k_{2}^{\prime}, k_{3}}\right)^{2}+\left(\Delta \epsilon_{k, k_{3}^{\prime}, k_{3}, k_{2}}\right)^{2}}\right) \\
& \times\left(n_{k_{3}^{\prime}}-n_{k_{3}}\right) \delta_{k+k_{3}, k_{3}^{\prime}+k_{2}} \\
& +\frac{U^{2}}{\Omega^{2}} \sum_{k_{3}^{\prime}, k_{3}} \frac{\Delta \epsilon_{k_{3}, k_{1}, k_{3}^{\prime}, k_{2}}}{\Delta \epsilon_{k, k_{3}, k_{2}^{\prime}, k_{3}^{\prime}}}\left(\frac{1}{\left(\Delta \epsilon_{k_{3}, k_{1}, k_{3}^{\prime}, k_{2}}\right)^{2}}-\frac{1}{\left(\Delta \epsilon_{k_{3}, k_{1}, k_{3}^{\prime}, k_{2}}\right)^{2}+\left(\Delta \epsilon_{k, k_{3}, k_{2}^{\prime}, k_{3}^{\prime}}\right)^{2}}\right) \\
& \times\left(\left(1-n_{k_{3}^{\prime}}\right)\left(1-n_{k_{3}}\right)-n_{k_{3}^{\prime}} n_{k_{3}}\right) \delta_{k+k_{2}^{\prime}, k_{3}+k_{3}^{\prime}} \\
& +O\left(U^{3}\right) \text {, } \\
& \tilde{G}_{k, k_{1}, k_{2}^{\prime}, k_{2}}=\frac{U^{2}}{\Omega^{2}} \sum_{k_{3}^{\prime}, k_{3}} \frac{\Delta \epsilon_{k_{3}^{\prime}, k_{3}, k_{2}^{\prime}, k_{2}}}{\Delta \epsilon_{k, k_{1}, k_{3}, k_{3}^{\prime}}}\left(\frac{1}{\left(\Delta \epsilon_{k_{3}^{\prime}, k_{3}, k_{2}^{\prime}, k_{2}}\right)^{2}+\left(\Delta \epsilon_{k, k_{1}, k_{3}, k_{3}^{\prime}}\right)^{2}}-\frac{1-e^{-\left(\Delta \epsilon_{k_{3}^{\prime}, k_{3}, k_{2}^{\prime}, k_{2}}\right)^{2} B}}{\left(\Delta \epsilon_{k_{3}^{\prime}, k_{3}, k_{2}^{\prime}, k_{2}}\right)^{2}}\right) \\
& \times\left(n_{k_{3}^{\prime}}-n_{k_{3}}\right) \delta_{k_{2}^{\prime}+k_{3}^{\prime}, k_{2}+k_{3}} \\
& +\frac{U^{2}}{\Omega^{2}} \sum_{k_{3}^{\prime}, k_{3}} \frac{\left(\Delta \epsilon_{k, k_{1}, k_{2}^{\prime}, k_{2}}\right)\left(\Delta \epsilon_{k, k_{1}, k_{3}^{\prime}, k_{3}}-\Delta \epsilon_{k_{2}^{\prime}, k_{2}, k_{3}, k_{3}^{\prime}}\right)}{\left(\Delta \epsilon_{k, k_{1}, k_{3}^{\prime}, k_{3}}\right)^{2}+\left(\Delta \epsilon_{k_{2}^{\prime}, k_{2}, k_{3}, k_{3}^{\prime}}\right)^{2}-\left(\Delta \epsilon_{k, k_{1}, k_{2}^{\prime}, k_{2}}\right)^{2}} \\
& \times\left(\frac{1}{\left(\Delta \epsilon_{k, k_{1}, k_{3}^{\prime}, k_{3}}\right)^{2}+\left(\Delta \epsilon_{k_{2}^{\prime}, k_{2}, k_{3}, k_{3}^{\prime}}\right)^{2}}-\frac{1}{\left(\Delta \epsilon_{k, k_{1}, k_{2}^{2}, k_{2}}\right)^{2}}\right) \\
& \times\left(n_{k_{3}^{\prime}}-n_{k_{3}}\right) \delta_{k_{3}^{\prime}+k_{2}, k_{3}+k_{2}^{\prime}} \\
& +O\left(U^{3}\right) \text {. }
\end{aligned}
$$


Now, we can calculate the time evolution of the annihilation operator at $B=\infty$, which is governed by the Hamiltonian $\tilde{H}$ from eq. (5.33).

\subsection{Time evolution of the annihilation operator}

At $B=\infty$, the HeISENBERg equation of motion for the transformed annihilation operator reads

$$
\frac{\mathrm{d} \tilde{\boldsymbol{c}}_{k \uparrow}(t)}{\mathrm{d} t}=i\left[\tilde{\boldsymbol{H}}, \tilde{\boldsymbol{c}}_{k \uparrow}(t)\right]_{-}
$$

As already mentioned, up to and including the time scale that is covered by our approach, $t \sim$ $\rho_{\mathrm{F}}^{-1} U^{-2}$, we can omit the elastic collisions in eq. 5.33 . Because the remaining Hamiltonian is quadratic in the annihilation and creation operators, we simply get

$$
\begin{aligned}
\tilde{h}_{k}(t) & =e^{-i \tilde{\epsilon}_{k} t} \tilde{h}_{k}, \\
\tilde{F}_{k, k_{1}, k_{2}^{\prime}, k_{2}}(t) & =e^{-i\left(\tilde{\epsilon}_{k_{1}}-\tilde{\epsilon}_{k_{2}^{\prime}}+\tilde{\epsilon}_{k_{2}}\right) t} \tilde{F}_{k, k_{1}, k_{2}^{\prime}, k_{2}}, \\
\tilde{G}_{k, k_{1}, k_{2}^{\prime}, k_{2}}(t) & =e^{-i\left(\tilde{\epsilon}_{k_{1}}-\tilde{\epsilon}_{k_{2}^{\prime}}+\tilde{\epsilon}_{k_{2}}\right) t} \tilde{G}_{k, k_{1}, k_{2}^{\prime}, k_{2}} .
\end{aligned}
$$

\subsubsection{Backward transformation}

We want to calculate expectation values of observables that depend on the annihilation and creation operators in the original basis at $B=0$, because then the initial state $\left|\psi_{0}\right\rangle$ does not need to be transformed under the unitary flow. According to the forward-backward transformation scheme, presented in sec. 2.2.1 (cf. Fig. 2.3), we still have to calculate the backward flow of the annihilation operator after the time evolution.

The flow equations are just the same, given by eqs. (5.42) - (5.44). The only difference is that we have to integrate them the other way around from $B=\infty$ to $B=0$, where the initial values at $B=\infty$ are given in eqs. (5.53) - 5.55). The backward integration is done in the same perturbative manner like the forward integration and we finally get at $B=0$ :

$$
\begin{aligned}
h_{k}(t)= & e^{-i \tilde{\epsilon}_{k} t} \\
& -\frac{U^{2}}{\Omega^{2}} e^{-i \epsilon_{k} t} \sum_{k_{1}, k_{2}^{\prime}, k_{2}} \frac{1-e^{i\left(\Delta \epsilon_{k, k_{1}, k_{2}^{\prime}, k_{2}}\right) t}}{\left(\Delta \epsilon_{k, k_{1}, k_{2}^{\prime}, k_{2}}\right)^{2}} \\
& \times\left(\left(1-n_{k_{1}}\right) n_{k_{2}^{\prime}}\left(1-n_{k_{2}}\right)+n_{k_{1}}\left(1-n_{k_{2}^{\prime}}\right) n_{k_{2}}\right) \delta_{k+k_{2}^{\prime}, k_{1}+k_{2}} \\
& +O\left(U^{3}\right),
\end{aligned}
$$




$$
\begin{aligned}
& F_{k, k_{1}, k_{2}^{\prime}, k_{2}}(t)=\frac{U}{\Omega} e^{-i \epsilon_{k} t} \frac{1-e^{i\left(\Delta \epsilon_{k, k_{1}, k_{2}^{\prime}, k_{2}}\right) t}}{\Delta \epsilon_{k, k_{1}, k_{2}^{\prime}, k_{2}}} \\
& -\frac{U^{2}}{\Omega^{2}} e^{-i \epsilon_{k} t} \sum_{k_{3}, k_{4}} \frac{\Delta \epsilon_{k, k_{1}, k_{2}^{\prime}, k_{2}}\left(\Delta \epsilon_{k_{3}, k_{1}, k_{4}, k_{2}}-\Delta \epsilon_{k, k_{3}, k_{2}^{\prime}, k_{4}}\right)}{\left(\Delta \epsilon_{k, k_{1}, k_{2}^{\prime}, k_{2}}\right)^{2}-\left(\Delta \epsilon_{k_{3}, k_{1}, k_{4}, k_{2}}\right)^{2}-\left(\Delta \epsilon_{k, k_{3}, k_{2}^{\prime}, k_{4}}\right)^{2}} \\
& \times\left(\frac{e^{i\left(\Delta \epsilon_{k, k_{1}, k_{2}^{\prime}, k_{2}}\right) t}-1}{\left(\Delta \epsilon_{k_{3}, k_{1}, k_{4}, k_{2}}\right)^{2}+\left(\Delta \epsilon_{k, k_{3}, k_{2}^{\prime}, k_{4}}\right)^{2}}-\frac{e^{i\left(\Delta \epsilon_{k, k_{1}, k_{2}^{\prime}, k_{2}}\right) t}-1}{\left(\Delta \epsilon_{k, k_{1}, k_{2}^{\prime}, k_{2}}\right)^{2}}\right) \\
& \times\left(n_{k_{3}} n_{k_{4}}-\left(1-n_{k_{3}}\right)\left(1-n_{k_{4}}\right)\right) \delta_{k+k_{2}^{\prime}, k_{3}+k_{4}} \\
& +\frac{U^{2}}{\Omega^{2}} e^{-i \epsilon_{k} t} \sum_{k_{3}, k_{4}} \frac{\Delta \epsilon_{k, k_{1}, k_{2}^{\prime}, k_{2}}\left(\Delta \epsilon_{k_{3}, k_{1}, k_{2}^{\prime}, k_{4}}-\Delta \epsilon_{k, k_{3}, k_{4}, k_{2}}\right)}{\left(\Delta \epsilon_{k, k_{1}, k_{2}, k_{2}}\right)^{2}-\left(\Delta \epsilon_{k_{3}, k_{1}, k_{2}^{\prime}, k_{4}}\right)^{2}-\left(\Delta \epsilon_{k, k_{3}, k_{4}, k_{2}}\right)^{2}} \\
& \times\left(\frac{e^{i\left(\Delta \epsilon_{k, k_{1}, k_{2}^{\prime}, k_{2}}\right) t}-1}{\left(\Delta \epsilon_{k_{3}, k_{1}, k_{2}^{\prime}, k_{4}}\right)^{2}+\left(\Delta \epsilon_{k, k_{3}, k_{4}, k_{2}}\right)^{2}}-\frac{e^{i\left(\Delta \epsilon_{k, k_{1}, k_{2}^{\prime}, k_{2}}\right) t}-1}{\left(\Delta \epsilon_{k, k_{1}, k_{2}^{\prime}, k_{2}}\right)^{2}}\right) \\
& \times\left(n_{k_{3}}-n_{k_{4}}\right) \delta_{k+k_{4}, k_{3}+k_{2}} \\
& +\frac{U^{2}}{\Omega^{2}} e^{-i \epsilon_{k} t} \sum_{k_{3}^{\prime}, k_{3}} \frac{\Delta \epsilon_{k_{3}^{\prime}, k_{1}, k_{2}^{\prime}, k_{3}}}{\Delta \epsilon_{k, k_{3}^{\prime}, k_{3}, k_{2}}} \\
& \times\left(\frac{e^{i\left(\Delta \epsilon_{k, k_{1}, k_{2}^{\prime}, k_{2}}\right) t}-e^{i\left(\Delta \epsilon_{k, k_{3}^{\prime}, k_{3}, k_{2}}\right) t}}{\left(\Delta \epsilon_{k_{3}^{\prime}, k_{1}, k_{2}^{\prime}, k_{3}}\right)^{2}}-\frac{e^{i\left(\Delta \epsilon_{k, k_{1}, k_{2}^{\prime}, k_{2}}\right) t}-1}{\left(\Delta \epsilon_{k_{3}^{\prime}, k_{1}, k_{2}^{\prime}, k_{3}}\right)^{2}+\left(\Delta \epsilon_{k, k_{3}^{\prime}, k_{3}, k_{2}}\right)^{2}}\right) \\
& \times\left(n_{k_{3}^{\prime}}-n_{k_{3}}\right) \delta_{k+k_{3}, k_{3}^{\prime}+k_{2}} \\
& +\frac{U^{2}}{\Omega^{2}} e^{-i \epsilon_{k} t} \sum_{k_{3}^{\prime}, k_{3}} \frac{\Delta \epsilon_{k_{3}, k_{1}, k_{3}^{\prime}, k_{2}}}{\Delta \epsilon_{k, k_{3}, k_{2}^{\prime}, k_{3}^{\prime}}}
\end{aligned}
$$

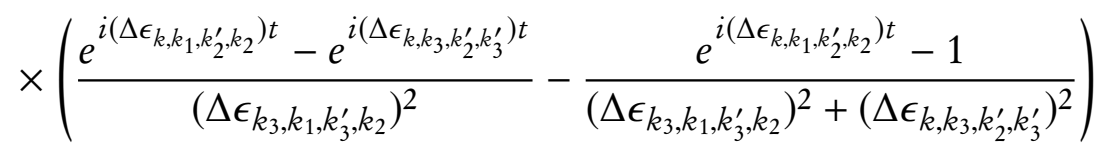

$$
\begin{aligned}
& \times\left(\left(1-n_{k_{3}^{\prime}}\right)\left(1-n_{k_{3}}\right)-n_{k_{3}^{\prime}} n_{k_{3}}\right) \delta_{k+k_{2}^{\prime}, k_{3}+k_{3}^{\prime}} \\
& +O\left(U^{3}\right) \\
& G_{k, k_{1}, k_{2}^{\prime}, k_{2}}(t)=-\frac{U^{2}}{\Omega^{2}} e^{-i \epsilon_{k} t} \sum_{k_{3}^{\prime}, k_{3}} \frac{\Delta \epsilon_{k_{3}^{\prime}, k_{3}, k_{2}^{\prime}, k_{2}}}{\Delta \epsilon_{k, k_{1}, k_{3}, k_{3}^{\prime}}} \\
& \times\left(\frac{1-e^{i\left(\Delta \epsilon_{k, k_{1}, k_{2}^{\prime}, k_{2}}\right) t}}{\left(\Delta \epsilon_{k_{3}^{\prime}, k_{3}, k_{2}^{\prime}, k_{2}}\right)^{2}+\left(\Delta \epsilon_{k, k_{1}, k_{3}, k_{3}^{\prime}}\right)^{2}}-\frac{e^{i\left(\Delta \epsilon_{k, k_{1}, k_{3}, k_{3}^{\prime}}\right) t}-e^{i\left(\Delta \epsilon_{k, k_{1}, k_{2}^{\prime}, k_{2}}\right) t}}{\left(\Delta \epsilon_{k_{3}^{\prime}, k_{3}, k_{2}^{\prime}, k_{2}}\right)^{2}}\right) \\
& \times\left(n_{k_{3}^{\prime}}-n_{k_{3}}\right) \delta_{k_{2}^{\prime}+k_{3}^{\prime}, k_{2}+k_{3}} \\
& +\frac{U^{2}}{\Omega^{2}} e^{-i \epsilon_{k} t} \sum_{k_{3}^{\prime}, k_{3}} \frac{\left(\Delta \epsilon_{k, k_{1}, k_{2}^{\prime}, k_{2}}\right)\left(\Delta \epsilon_{k, k_{1}, k_{3}^{\prime}, k_{3}}-\Delta \epsilon_{k_{2}^{\prime}, k_{2}, k_{3}, k_{3}^{\prime}}\right)}{\left(\Delta \epsilon_{k, k_{1}, k_{3}^{\prime}, k_{3}}\right)^{2}+\left(\Delta \epsilon_{k_{2}^{\prime}, k_{2}, k_{3}, k_{3}^{\prime}}\right)^{2}-\left(\Delta \epsilon_{k, k_{1}, k_{2}^{\prime}, k_{2}}\right)^{2}} \\
& \times\left(\frac{e^{i\left(\Delta \epsilon_{k, k_{1}, k_{2}^{\prime}, k_{2}}\right) t}-1}{\left(\Delta \epsilon_{k, k_{1}, k_{3}^{\prime}, k_{3}}\right)^{2}+\left(\Delta \epsilon_{k_{2}^{\prime}, k_{2}, k_{3}, k_{3}^{\prime}}\right)^{2}}-\frac{e^{i\left(\Delta \epsilon_{k, k_{1}, k_{2}^{\prime}, k_{2}}\right) t}-1}{\left(\Delta \epsilon_{k, k_{1}, k_{2}^{\prime}, k_{2}}\right)^{2}}\right) \\
& \times\left(n_{k_{3}^{\prime}}-n_{k_{3}}\right) \delta_{k_{3}^{\prime}+k_{2}, k_{3}+k_{2}^{\prime}} \\
& +O\left(U^{3}\right)
\end{aligned}
$$


This result is the perturbative solution of the HEISENBERG equation of motion for the annihilation operator that has a structure given by eq. (5.36).

The difference to the calculation by Moeckel and KeHreIN [85] up to this point lies in the evaluation of the second-order contributions to $F_{k, k_{1}, k_{2}^{\prime}, k_{2}}(t)$ and $G_{k, k_{1}, k_{2}^{\prime}, k_{2}}(t)$. They are important for the calculation of density-density correlations.

\section{Consistency check: preservation of the canonical anti-commutation relation}

The sequence of forward flow, time evolution and backward flow, which was applied to the annihilation operator, only consists of unitary transformations. Hence, the canonical anticommutation relation

$$
\left[c_{k \uparrow}(t), c_{k^{\prime} \uparrow}^{\dagger}(t)\right]_{+} \stackrel{!}{=} \delta_{k, k^{\prime}}+O\left(U^{3}\right)
$$

should be preserved, at least in a perturbative manner. In App. B.1 we show that this consistency condition leads to a relation between $h_{k}(t), F_{k, k_{1}, k_{2}^{\prime}, k_{2}}(t)$ and $G_{k, k_{1}, k_{2}^{\prime}, k_{2}}(t)$ that is indeed fulfilled by the solutions from eqs. 5.56 - 5.58.

\subsection{Time scale analysis}

We will analyze the stability of our perturbative expansion by considering the second-order correction term for $h_{k}(t)$ from eq. (5.56),

$$
\begin{aligned}
\Delta h_{k}(t)=\frac{U^{2}}{\Omega^{2}} e^{-i \epsilon_{k} t} \sum_{k_{1}, k_{2}^{\prime}, k_{2}} \frac{e^{i\left(\Delta \epsilon_{k, k_{1}, k_{2}^{\prime}, k_{2}}\right) t}-1}{\left(\Delta \epsilon_{k, k_{1}, k_{2}^{\prime}, k_{2}}\right)^{2}} \\
\quad \times\left(\left(1-n_{k_{1}}\right) n_{k_{2}^{\prime}}\left(1-n_{k_{2}}\right)+n_{k_{1}}\left(1-n_{k_{2}^{\prime}}\right) n_{k_{2}}\right) \delta_{k+k_{2}^{\prime}, k_{1}+k_{2}} .
\end{aligned}
$$

We introduce energy integrals and assume the DMFT limit, where $\delta_{k+k_{2}^{\prime}, k_{1}+k_{2}} \rightarrow \Omega^{-1}$ (cf. sec. 3.2.2),

$$
\begin{aligned}
& \Delta h_{k}(t)=U^{2} e^{-i \epsilon_{k} t} \int \mathrm{d} \epsilon_{1} \int \mathrm{d} \epsilon_{2^{\prime}} \int \mathrm{d} \epsilon_{2} D\left(\epsilon_{1}\right) D\left(\epsilon_{2^{\prime}}\right) D\left(\epsilon_{2}\right) \\
& \times \frac{e^{i\left(\Delta \epsilon_{k, 1,2^{\prime}, 2}\right) t}-1}{\left(\Delta \epsilon_{k, 1,2^{\prime}, 2}\right)^{2}}\left(\left(1-n_{1}\right) n_{2^{\prime}}\left(1-n_{2}\right)+n_{1}\left(1-n_{2^{\prime}}\right) n_{2}\right) .
\end{aligned}
$$

Next, we substitute $E=\epsilon_{1}-\epsilon_{2^{\prime}}+\epsilon_{2}$ and get

$$
\Delta h_{k}(t)=U^{2} e^{-i \epsilon_{k} t} \int \mathrm{d} E \frac{e^{i\left(\epsilon_{k}-E\right) t}-1}{\left(\epsilon_{k}-E\right)^{2}} I(E),
$$

with a phase space factor

$$
\begin{aligned}
I(E)=\int \mathrm{d} \epsilon_{1} \int \mathrm{d} \epsilon_{2^{\prime}} \int \mathrm{d} \epsilon_{2} D\left(\epsilon_{1}\right) D\left(\epsilon_{2^{\prime}}\right) D\left(\epsilon_{2}\right)\left(1-n_{1}\right)\left(1-n_{2^{\prime}}\right)\left(1-n_{2}\right) \\
\times\left(\delta\left(\epsilon_{1}+\epsilon_{2^{\prime}}+\epsilon_{2}-E\right)+\delta\left(\epsilon_{1}+\epsilon_{2^{\prime}}+\epsilon_{2}+E\right)\right) .
\end{aligned}
$$


At zero temperature and for a constant density of states

$$
D(\epsilon)= \begin{cases}\rho_{\mathrm{F}}, & \text { for }|\epsilon| \leq \frac{\Delta}{2} \\ 0, & \text { for }|\epsilon|>\frac{\Delta}{2}\end{cases}
$$

which is the zeroth order approximation around the Fermi level, with $\Delta^{-1} \equiv \rho_{\mathrm{F}}$ being the density of states directly at the FERMI surface, the phase space factor

$$
I(E)=\rho_{\mathrm{F}}^{3} \int_{0}^{\Delta / 2} \mathrm{~d} \epsilon_{1} \int_{0}^{\Delta / 2} \mathrm{~d} \epsilon_{2^{\prime}} \int_{0}^{\Delta / 2} \mathrm{~d} \epsilon_{2} \delta\left(\epsilon_{1}+\epsilon_{2^{\prime}}+\epsilon_{2}-|E|\right)=\rho_{\mathrm{F}}^{3} \int_{x+y+z=|E|} \frac{\mathrm{d} \sigma}{\sqrt{3}}
$$

is the surface integral over an equilateral triangle with side length $\sqrt{2}|E|$, and Hence

$$
I(E)=\rho_{\mathrm{F}}^{3} \frac{E^{2}}{2} .
$$

Then, the correction term for $h_{k}(t)$ is

$$
\Delta h_{k}(t)=\rho_{\mathrm{F}}^{3} \frac{U^{2}}{2} e^{-i \epsilon_{k} t} \int_{-\frac{\Delta}{2}}^{\frac{\Delta}{2}} \mathrm{~d} E \frac{e^{i\left(\epsilon_{k}-E\right) t}-1}{\left(\epsilon_{k}-E\right)^{2}} E^{2} .
$$

The integral only converges at the Fermi surface, where $\epsilon_{k}=0$. Away from the Fermi surface, the integral has a pole and hence formally diverges. But, we can rewrite it as

$$
\begin{aligned}
\int_{-\frac{\Delta}{2}}^{\frac{\Delta}{2}} \mathrm{~d} E \frac{e^{i\left(\epsilon_{k}-E\right) t}-1}{\left(\epsilon_{k}-E\right)^{2}} E^{2} & =\int_{-\frac{\Delta}{2}-\epsilon_{k}}^{\frac{\Delta}{2}-\epsilon_{k}} \mathrm{~d} E \frac{e^{-i E t}-1}{E^{2}}\left(E+\epsilon_{k}\right)^{2} \\
& =\int_{-\frac{\Delta}{2}-\epsilon_{k}}^{\frac{\Delta}{2}-\epsilon_{k}} \mathrm{~d} E \frac{\cos (E t)-i \sin (E t)-1}{E^{2}}\left(E^{2}+2 E \epsilon_{k}+\epsilon_{k}^{2}\right) .
\end{aligned}
$$

For $0<\epsilon_{k}<\frac{\Delta}{2}$, we can split the integral as follows,

$$
\begin{aligned}
\int_{-\frac{\Delta}{2}}^{\frac{\Delta}{2}} \mathrm{~d} E \frac{e^{i\left(\epsilon_{k}-E\right) t}-1}{\left(\epsilon_{k}-E\right)^{2}} E^{2}= & \int_{-\frac{\Delta}{2}-\epsilon_{k}}^{-\frac{\Delta}{2}+\epsilon_{k}} \mathrm{~d} E \frac{\cos (E t)-i \sin (E t)-1}{E^{2}}\left(E^{2}+2 E \epsilon_{k}+\epsilon_{k}^{2}\right) \\
& +\int_{-\frac{\Delta}{2}+\epsilon_{k}}^{\frac{\Delta}{2}-\epsilon_{k}} \mathrm{~d} E \frac{\cos (E t)-i \sin (E t)-1}{E^{2}}\left(E^{2}+2 E \epsilon_{k}+\epsilon_{k}^{2}\right) .
\end{aligned}
$$

Only the latter part contains the pole $(E=0)$, but the integration area is symmetric around it. So, we can omit the antisymmetric parts of the integrand,

$$
\begin{aligned}
\int_{-\frac{\Delta}{2}}^{\frac{\Delta}{2}} \mathrm{~d} E \frac{e^{i\left(\epsilon_{k}-E\right) t}-1}{\left(\epsilon_{k}-E\right)^{2}} E^{2}= & \int_{-\frac{\Delta}{2}-\epsilon_{k}}^{-\frac{\Delta}{2}+\epsilon_{k}} \mathrm{~d} E \frac{\cos (E t)-i \sin (E t)-1}{E^{2}}\left(E^{2}+2 E \epsilon_{k}+\epsilon_{k}^{2}\right) \\
& +2 \int_{0}^{\frac{\Delta}{2}-\epsilon_{k}} \mathrm{~d} E\left(\cos (E t)-1+\frac{\cos (E t)-1}{E^{2}} \epsilon_{k}^{2}-2 i \epsilon_{k} \frac{\sin (E t)}{E}\right) \\
= & \int_{-\frac{\Delta}{2}-\epsilon_{k}}^{-\frac{\Delta}{2}+\epsilon_{k}} \mathrm{~d} E \frac{\cos (E t)-i \sin (E t)-1}{E^{2}}\left(E^{2}+2 E \epsilon_{k}+\epsilon_{k}^{2}\right) \\
& +2 \int_{0}^{\frac{\Delta}{2}-\epsilon_{k}} \mathrm{~d} E\left(\cos (E t)-1+\frac{\cos (E t)-1}{E^{2}} \epsilon_{k}^{2}-2 i \epsilon_{k} \frac{\sin (E t)}{E}\right) .
\end{aligned}
$$


These integrals are well-defined. To analyze the time-dependence, we substitute $\phi \stackrel{\text { def }}{=} E t$,

$$
\begin{aligned}
\int_{-\frac{\Delta}{2}}^{\frac{\Delta}{2}} \mathrm{~d} E \frac{e^{i\left(\epsilon_{k}-E\right) t}-1}{\left(\epsilon_{k}-E\right)^{2}} E^{2}= & \int_{-\frac{\Delta}{2} t-\epsilon_{k} t}^{-\frac{\Delta}{2} t+\epsilon_{k} t} \mathrm{~d} \phi \frac{\cos (\phi)-i \sin (\phi)-1}{\phi^{2}}\left(\phi^{2}+2 \phi \epsilon_{k} t+\epsilon_{k}^{2} t^{2}\right) \frac{1}{t} \\
& +2 \int_{0}^{\frac{\Delta}{2} t-\epsilon_{k} t} \mathrm{~d} \phi\left(\cos (\phi)-1+\frac{\cos (\phi)-1}{\phi^{2}} \epsilon_{k}^{2} t^{2}-2 i \frac{\sin (\phi)}{\phi} \epsilon_{k} t\right) \frac{1}{t} .
\end{aligned}
$$

In the long-time limit, the leading term is

$$
\begin{aligned}
\int_{-\frac{\Delta}{2}}^{\frac{\Delta}{2}} \mathrm{~d} E \frac{e^{i\left(\epsilon_{k}-E\right) t}-1}{\left(\epsilon_{k}-E\right)^{2}} E^{2} \approx & \int_{-\frac{\Delta}{2} t-\epsilon_{k} t}^{\frac{\Delta}{2} t-\epsilon_{k} t} \mathrm{~d} \phi \frac{\cos (\phi)}{\phi^{2}} \epsilon_{k}^{2} t+O\left(t^{0}\right) \\
\approx & {\left[-\frac{\cos (\phi)}{\phi}-\operatorname{SinIntegral}(\phi)\right]_{-\frac{\Delta}{2} t-\epsilon_{k} t}^{\frac{\Delta}{2} t-\epsilon_{k} t} \cdot \epsilon_{k}^{2} t+O\left(t^{0}\right) } \\
\approx & -\left[\operatorname{SinIntegral}\left(\left(\frac{\Delta}{2}-\epsilon_{k}\right) t\right)+\operatorname{SinIntegral}\left(\left(\frac{\Delta}{2}+\epsilon_{k}\right) t\right)\right] \epsilon_{k}^{2} t \\
& +O\left(t^{0}\right) \\
\approx & -\pi \epsilon_{k}^{2} t .
\end{aligned}
$$

Hence, the second order correction term for $h_{k}(t)$ becomes comparable to 1 for times

$$
t \sim \frac{1}{\rho_{\mathrm{F}}^{3} U^{2} \epsilon_{k}^{2}} .
$$

This implies that the perturbative nature of our approach is valid for times $t \lesssim \rho_{\mathrm{F}}^{-1} U^{-2}$ for a worst case estimate. However, one often considers only contributions in the vicinity of the FERMI level, $\epsilon_{k} \approx 0$, which much improves the stability of the time evolution.

At finite temperature $T$, the FERMI-Dirac distributions in eq. (5.63) are smeared out in a domain $\propto T$. Therefore, the phase space factor gets a correction term $\propto T^{2}$, so that $h_{k}(t)$ becomes comparable to 1 for times

$$
t \sim \min \left\{\left(\frac{1}{\rho_{\mathrm{F}}^{3} U^{2} \epsilon_{k}^{2}}\right),\left(\frac{1}{\rho_{\mathrm{F}}^{3} U^{2} T^{2}}\right)\right\} .
$$

This does not change the worst case estimate from above. 


\section{Chapter 6}

\section{Build-up of density-density correlations}

With the results from the preceding chapter, i.e., with the annihilation operator of the HuBBARD model evolved in time, we can turn towards the calculation of actual observables. Here, we draw on the quench setup because expectation values will be evaluated with respect to the initial state $\left|\psi_{0}\right\rangle$ defined by eq. (5.4), i.e.,

$$
O(t) \equiv\langle O(t)\rangle \equiv\left\langle\psi_{0}|O(t)| \psi_{0}\right\rangle .
$$

In previous work, one-particle functions, namely the momentum distribution function $n_{k \uparrow}(t)=$ $\left\langle c_{k \uparrow}^{\dagger}(t) c_{k \uparrow}(t)\right\rangle$, have been calculated [85]. With the results from eqs. $5.56-(5.58)$, we are now able to go one step further and calculate two-particle correlation functions. The most accessible ones are correlations between on-site particle densities $n_{x, \sigma}$, and hence we will analyse the density-density susceptibility

$$
\chi_{x^{\prime}, x}^{\sigma^{\prime} \sigma}(t) \stackrel{\text { def }}{=}-i\left\langle\left[\boldsymbol{n}_{x^{\prime}, \sigma^{\prime}}(t), \boldsymbol{n}_{x, \sigma}(0)\right]_{-}\right\rangle \equiv 2 \mathfrak{J}\left(\left\langle\boldsymbol{n}_{x^{\prime}, \sigma^{\prime}}(t) \boldsymbol{n}_{x, \sigma}(0)\right\rangle\right)
$$

and the equal-time connected density-density correlation function

$$
C_{x^{\prime}, x}^{\sigma^{\prime} \sigma}(t) \stackrel{\text { def }}{=}\left\langle\boldsymbol{n}_{x^{\prime}, \sigma^{\prime}}(t) \boldsymbol{n}_{x, \sigma}(t)\right\rangle-\left\langle\boldsymbol{n}_{x^{\prime}, \sigma^{\prime}}(t)\right\rangle\left\langle\boldsymbol{n}_{x, \sigma}(t)\right\rangle
$$

both of them in a leading-order expansion for small $U$. Here, the operator

$$
\boldsymbol{n}_{x, \sigma}(t) \stackrel{\text { def }}{=} \frac{1}{\Omega} \sum_{k^{\prime}, k} e^{i\left(k^{\prime}-k\right) x} \boldsymbol{c}_{k^{\prime} \sigma}^{\dagger}(t) \boldsymbol{c}_{k \sigma}(t)
$$

describes the density of spin- $\sigma$ particles at lattice site $x$.

The susceptibility is interpreted as the linear response of the particle density at lattice site $x^{\prime}$ at time $t$ to a small perturbation at lattice site $x$ at time $t=0$. For finite group velocities $v_{k}=\nabla_{k} \epsilon_{k}$, we expect the susceptibility to vanish for sufficiently large distances $x^{\prime}-x$ at a fixed time $t$ due to causality. In contrast the equal-time correlation function might be enhanced on arbitrary large distances by entanglement between particles in the initial state. In chapter 7 , we will analyze how the susceptibility and the equal-time correlation function decay for large distances $x^{\prime}-x$.

In our analysis, we will distinguish four cases that are depicted in Tab. 6.1. Both the susceptibility and the equal-time correlation function will be evaluated for antiparallel spins and 
for parallel spins respectively. Due to the symmetry of the HubBard model the case $\downarrow \uparrow$ is equivalent to $\uparrow \downarrow$ as well as $\downarrow \downarrow$ is equivalent to $\uparrow \uparrow$. We will see that for antiparallel spins both functions have first-order contributions in the expansion for small $U$, while for parallel spins the leading order is quadratic in $U$, apart from a zeroth-order contribution from the initial state.

\begin{tabular}{l|l|l} 
& anti-parallel spins $\uparrow \downarrow$ & parallel spins $\uparrow \uparrow$ \\
\hline \hline susceptibility $\chi_{x^{\prime}, x}(t)$ & $O(U)$ & $O\left(U^{2}\right)$ \\
\hline correlation function $C_{x^{\prime}, x}(t)$ & $O(U)$ & $O\left(U^{2}\right)$
\end{tabular}

Table 6.1: Leading order of the different density-density correlations

The first step in the calculation of the correlation functions from eqs. (6.2) and 6.3 lies in working out the general structure of the one-particle density operator $c_{k^{\prime} \sigma}^{\dagger} c_{k \sigma}$ what will be done in sec. 6.1.

Then, we will turn our attention towards the density-density susceptibility in sec. 6.2 and the equal-time connected density-density correlation function in sec. 6.3, where we will derive closed expressions for both antiparallel spins and parallel spins.

In sec. 6.4, the equal-time correlation functions will also be evaluated for the equilibrium HuBbard model by calculating the expectation values in the energy-diagonal basis from the flow equation analysis. This was explained in sec. 2.2.1 and we will compare the equilibrium values to the prethermalization values of the nonequilibrium functions derived before.

In order to analyze the long-range behavior of the correlation function for parallel spins $C_{x^{\prime}, x}^{\uparrow}(t)$, further approximations will be necessary. As pointed out in sec. 3.2, the limit of infinite spatial dimensions simplifies the analysis. In addition, we will transform the spatial correlation function to momentum space by means of a FOURIER transformation in sec. 6.5. The small-momentum behavior of the FOURIER transformed correlation function in infinite dimensions then provides information about the decay behavior in real space, which we will discuss in chapter 7 . 


\subsection{General structure of the one-particle density operator}

Let us start by calculating the product $c_{k^{\prime} \uparrow}^{\dagger}(t) c_{k \uparrow}(t)$, which will allow us to construct the susceptibility from eq. (6.2) and the equal-time connected correlation function from eq. (6.3). The general structure of the time-evolved annihilation operator can be read from eq. (5.36). It is given by

$$
\begin{aligned}
c_{k \uparrow}(t)= & h_{k}(t): c_{k \uparrow}: \\
& +\sum_{k_{1}, k_{2}^{\prime}, k_{2}} F_{k, k_{1}, k_{2}^{\prime}, k_{2}}(t): c_{k_{1} \uparrow} c_{k_{2}^{\prime} \downarrow}^{\dagger} c_{k_{2} \downarrow}: \delta_{k+k_{2}, k_{1}+k_{2}} \\
& +\sum_{k_{1}, k_{2}^{\prime}, k_{2}} G_{k, k_{1}, k_{2}^{\prime}, k_{2}}(t): c_{k_{1} \uparrow} c_{k_{2}^{\prime} \uparrow}^{\dagger} c_{k_{2} \uparrow}: \delta_{k+k_{2}, k_{1}+k_{2}} \\
& + \text { higher-order interaction terms },
\end{aligned}
$$

where

$$
h_{k}(t)=e^{-i \epsilon_{k} t}+O\left(U^{2}\right), \quad F_{k, k_{1}, k_{2}^{\prime}, k_{2}}(t)=O(U), \quad G_{k, k_{1}, k_{2}^{\prime}, k_{2}}(t)=O\left(U^{2}\right)
$$

and the higher-order interaction terms are at least $O\left(U^{2}\right)$. The explicit expressions for the coefficients are given in eqs. (5.56) - (5.58), but we are not yet using them.

In a second-order expansion, we get the following contributions for the product of the creation and the annihilation operator,

$$
\begin{aligned}
& c_{k^{\prime} \uparrow}^{\dagger}(t) c_{k \uparrow}(t)=h_{k^{\prime}}^{*}(t) h_{k}(t): c_{k^{\prime} \uparrow}^{\dagger}:: c_{k \uparrow}: \\
& +\sum_{k_{1}, k_{2}^{\prime}, k_{2}} h_{k^{\prime}}^{*}(t) F_{k, k_{1}, k_{2}^{\prime}, k_{2}}(t): c_{k^{\prime} \uparrow}^{\dagger}:: c_{k_{1} \uparrow} c_{k_{2}^{\prime} \downarrow}^{\dagger} c_{k_{2} \downarrow}: \delta_{k+k_{2}^{\prime}, k_{1}+k_{2}} \\
& +\sum_{k_{1}, k_{2}^{\prime}, k_{2}} h_{k^{\prime}}^{*}(t) G_{k, k_{1}, k_{2}^{\prime}, k_{2}}(t): c_{k^{\prime} \uparrow}^{\dagger}:: c_{k_{1} \uparrow} c_{k_{2}^{\prime} \uparrow}^{\dagger} c_{k_{2} \uparrow}: \delta_{k+k_{2}^{\prime}, k_{1}+k_{2}} \\
& +\sum_{k_{1}, k_{2}^{\prime}, k_{2}} F_{k^{\prime}, k_{1}, k_{2}^{\prime}, k_{2}}^{*}(t) h_{k}(t): c_{k_{2} \downarrow}^{\dagger} c_{k_{2}^{\prime} \downarrow} c_{k_{1} \uparrow}^{\dagger}:: c_{k \uparrow}: \delta_{k^{\prime}+k_{2}^{\prime}, k_{1}+k_{2}} \\
& +\sum_{k_{i}^{\prime}, k_{i}} F_{k^{\prime}, k_{1}, k_{2}^{\prime}, k_{2}}^{*}(t) F_{k, k_{1}^{\prime}, k_{3}^{\prime}, k_{3}}(t): c_{k_{2} \downarrow}^{\dagger} c_{k_{2}^{\prime} \downarrow} c_{k_{1} \uparrow}^{\dagger}:: c_{k_{1}^{\prime} \uparrow} c_{k_{3}^{\prime} \downarrow}^{\dagger} c_{k_{3} \downarrow}: \delta_{k^{\prime}+k_{2}^{\prime}, k_{1}+k_{2}} \delta_{k+k_{3}^{\prime}, k_{1}^{\prime}+k_{3}} \\
& +\sum_{k_{1}, k_{2}^{\prime}, k_{2}} G_{k^{\prime}, k_{1}, k_{2}^{\prime}, k_{2}}^{*}(t) h_{k}(t): c_{k_{2} \uparrow}^{\dagger} c_{k_{2}^{\prime} \uparrow} c_{k_{1} \uparrow}^{\dagger}:: c_{k \uparrow}: \delta_{k^{\prime}+k_{2}^{\prime}, k_{1}+k_{2}} \\
& + \text { "irrelevant terms" } \\
& +O\left(U^{3}\right) \text {. }
\end{aligned}
$$

Here, the "irrelevant terms" are products of $h_{k}(t): c_{k \uparrow}:$ and the higher-order interaction terms from eq. (6.5). If we calculated these products of normal-ordered expressions, the solutions would be at least quartic in the annihilation and creation operators and of second order in $U$. We will see that they are indeed irrelevant for the calculation of the density-density correlations in the sense that they do not contribute in a second-order expansion.

Next, we calculate the products of the normal ordered expressions in the equation above using 
Wick's second theorem (cf. App. A.3). In order to keep the overview in the calculation of the equal-time correlation function in sec. 6.3, we will mark terms that have the same operator structure by the same color. Thus, we get

$$
\begin{aligned}
& c_{k^{\prime} \uparrow}^{\dagger}(t) c_{k \uparrow}(t)=\left|h_{k^{\prime}}(t)\right|^{2} n_{k^{\prime}} \delta_{k^{\prime}, k}+\sum_{k_{1}, k_{2}^{\prime}, k_{2}}\left|F_{k^{\prime}, k_{1}, k_{2}^{\prime}, k_{2}}(t)\right|^{2} n_{k_{1}}\left(1-n_{k_{2}^{\prime}}\right) n_{k_{2}} \delta_{k^{\prime}+k_{2}^{\prime}, k_{1}+k_{2}} \delta_{k^{\prime}, k} \\
& +h_{k^{\prime}}^{*}(t) h_{k}(t): c_{k^{\prime} \uparrow}^{\dagger} c_{k \uparrow}: \\
& +\sum_{k_{i}^{\prime}, k_{i}} F_{k^{\prime}, k_{1}^{\prime}, k_{2}^{\prime}, k_{2}}^{*}(t) F_{k, k_{1}, k_{2}^{\prime}, k_{2}}(t)\left(1-n_{k_{2}^{\prime}}\right) n_{k_{2}}: c_{k_{1}^{\prime} \uparrow}^{\dagger} c_{k_{1} \uparrow}: \delta_{k+k_{2}^{\prime}, k_{1}+k_{2}} \delta_{k^{\prime}+k_{1}, k+k_{1}^{\prime}} \\
& +\sum_{k_{1}^{\prime}, k_{1}} h_{k^{\prime}}^{*}(t)\left(G_{k, k^{\prime}, k_{1}^{\prime}, k_{1}}(t)-G_{k, k_{1}, k_{1}^{\prime}, k^{\prime}}(t)\right) n_{k^{\prime}}: c_{k_{1}^{\prime} \uparrow}^{\dagger} c_{k_{1} \uparrow}: \delta_{k^{\prime}+k_{1}, k+k_{1}^{\prime}} \\
& +\sum_{k_{1}^{\prime}, k_{1}} h_{k}(t)\left(G_{k^{\prime}, k, k_{1}, k_{1}^{\prime}}^{*}(t)-G_{k^{\prime}, k_{1}^{\prime}, k_{1}, k}^{*}(t)\right) n_{k}: c_{k_{1}^{\prime} \uparrow}^{\dagger} c_{k_{1} \uparrow}: \delta_{k^{\prime}+k_{1}, k+k_{1}^{\prime}} \\
& +\sum_{k_{1}^{\prime}, k_{1}} h_{k^{\prime}}^{*}(t) F_{k, k^{\prime}, k_{1}^{\prime}, k_{1}}(t) n_{k^{\prime}}: c_{k_{1}^{\prime} \downarrow}^{\dagger} c_{k_{1} \downarrow}: \delta_{k^{\prime}+k_{1}, k+k_{1}^{\prime}} \\
& +\sum_{k_{1}^{\prime}, k_{1}} h_{k}(t) F_{k^{\prime}, k, k_{1}, k_{1}^{\prime}}^{*}(t) n_{k}: c_{k_{1}^{\prime} \downarrow}^{\dagger} c_{k_{1} \downarrow}: \delta_{k^{\prime}+k_{1}, k+k_{1}^{\prime}} \\
& +\sum_{k_{i}^{\prime}, k_{i}} F_{k^{\prime}, k_{2}, k_{2}^{\prime}, k_{1}^{\prime}}^{*}(t) F_{k, k_{2}, k_{2}^{\prime}, k_{1}}(t)\left(1-n_{k_{2}^{\prime}}\right) n_{k_{2}}: c_{k_{1}^{\prime} \downarrow}^{\dagger} c_{k_{1} \downarrow}: \delta_{k+k_{2}^{\prime}, k_{1}+k_{2}} \delta_{k^{\prime}+k_{1}, k+k_{1}^{\prime}} \\
& -\sum_{k_{i}^{\prime}, k_{i}} F_{k^{\prime}, k_{2}^{\prime}, k_{1}, k_{2}}^{*}(t) F_{k, k_{2}^{\prime}, k_{1}^{\prime}, k_{2}}(t) n_{k_{2}^{\prime} n_{k_{2}}}: c_{k_{1}^{\prime} \downarrow}^{\dagger} c_{k_{1} \downarrow}: \delta_{k^{\prime}+k_{1}, k_{2}^{\prime}+k_{2}} \delta_{k^{\prime}+k_{1}, k+k_{1}^{\prime}} \\
& +\sum_{k_{1}, k_{2}^{\prime}, k_{2}} h_{k^{\prime}}^{*}(t) F_{k, k_{1}, k_{2}^{\prime}, k_{2}}(t): c_{k^{\prime} \uparrow}^{\dagger} c_{k_{1} \uparrow} c_{k_{2}^{\prime} \downarrow}^{\dagger} c_{k_{2} \downarrow}: \delta_{k+k_{2}^{\prime}, k_{1}+k_{2}} \\
& +\sum_{k_{1}^{\prime}, k_{2}^{\prime}, k_{2}} h_{k}(t) F_{k^{\prime}, k_{1}^{\prime}, k_{2}, k_{2}^{\prime}}^{*}(t): c_{k_{1}^{\prime} \uparrow}^{\dagger} c_{k \uparrow} c_{k_{2}^{\prime} \downarrow}^{\dagger} c_{k_{2} \downarrow}: \delta_{k_{1}^{\prime}+k_{2}^{\prime}, k^{\prime}+k_{2}} \\
& \text { + "irrelevant terms" } \\
& +O\left(U^{3}\right)
\end{aligned}
$$

where all normal-ordered expressions that contain at least four annihilation and creation operators and that are of second order in $U$ were shifted into the "irrelevant terms". From the contractions we got the factors $n_{k}$, which are given by the initial condition of the pre-quench state $\left|\psi_{0}\right\rangle$ in eq. (5.4).

\section{Consistency check: total spin-up particle number}

At this point, we can check whether the total spin-up particle number

$$
N_{\uparrow}(t) \stackrel{\text { def }}{=} \sum_{k} c_{k \uparrow}^{\dagger}(t) c_{k \uparrow}(t)
$$

is conserved. In App. B.2 we show that this operator commutes with the HubBard Hamiltonian by definition. Hence, it should be time-independent. Indeed, we find that the black 
and red terms in eq. (6.7) are consistent with this in the sense that a summation over $k^{\prime}=k$ results in a conserved quantity.

\subsection{Susceptibility}

The density-density susceptibilty from eq. 6.2,

$$
\begin{aligned}
\chi_{x^{\prime}, x}^{\sigma^{\prime} \sigma}(t) & =2 \mathfrak{J}\left(\left\langle\boldsymbol{n}_{x^{\prime}, \sigma^{\prime}}(t) \boldsymbol{n}_{x, \sigma}(0)\right\rangle\right) \\
& =\frac{2}{\Omega^{2}} \sum_{k^{\prime}, k, q^{\prime}, q} \mathfrak{J}\left(e^{i\left(k^{\prime}-k\right) x^{\prime}} e^{i\left(q^{\prime}-q\right) x}\left\langle\boldsymbol{c}_{k^{\prime} \sigma^{\prime}}^{\dagger}(t) \boldsymbol{c}_{k \sigma^{\prime}}(t) \boldsymbol{c}_{q^{\prime} \sigma^{\prime}}^{\dagger}(0) \boldsymbol{c}_{q \sigma}(0)\right\rangle\right)
\end{aligned}
$$

is easy to calculate because the second operator in the expression, $\boldsymbol{n}_{x, \sigma}(0)$, is evaluated at time $t=0$, where eq. (6.7) reduces to

$$
c_{q^{\prime} \sigma}^{\dagger}(0) c_{q \sigma}(0)=n_{q^{\prime}} \delta_{q^{\prime}, q^{+}}+c_{q^{\prime} \sigma}^{\dagger} c_{q \sigma}:
$$

In the evaluation of $\left\langle\boldsymbol{c}_{k^{\prime} \sigma^{\prime}}^{\dagger}(t) \boldsymbol{c}_{k \sigma^{\prime}}(t) \boldsymbol{c}_{q^{\prime} \sigma}^{\dagger}(0) \boldsymbol{c}_{q \sigma}(0)\right\rangle$ only full contractions of products of normalordered expressions survive (cf. App. A.4), hence we can neglect the orange terms in eq. 6.7).

\subsubsection{For antiparallel spins}

For antiparallel spins, the susceptibility has contributions of first order in $U$, so we can drop the second-order terms for a leading-order expansion. The terms from eq. (6.7) that completely contract with the operator $c_{q^{\prime} \downarrow}^{\dagger}(0) c_{q \downarrow}(0)$ from eq. 6.10 in first order are only

$$
\begin{aligned}
\boldsymbol{c}_{k^{\prime} \uparrow}^{\dagger}(t) \boldsymbol{c}_{k \uparrow}(t)= & \left|h_{k^{\prime}}(t)\right|^{2} n_{k^{\prime}} \delta_{k^{\prime}, k}+\sum_{k_{1}, k_{2}^{\prime}, k_{2}}\left|F_{k^{\prime}, k_{1}, k_{2}^{\prime}, k_{2}}(t)\right|^{2} n_{k_{1}}\left(1-n_{k_{2}^{\prime}}\right) n_{k_{2}} \delta_{k^{\prime}+k_{2}^{\prime}, k_{1}+k_{2}} \delta_{k^{\prime}, k} \\
& +\sum_{k_{1}^{\prime}, k_{1}} h_{k^{\prime}}^{*}(t) F_{k^{\prime}, k^{\prime}, k_{1}^{\prime}, k_{1}}(t) n_{k^{\prime}}: c_{k_{1}^{\prime} \downarrow}^{\dagger} c_{k_{1} \downarrow}: \delta_{k^{\prime}+k_{1}, k+k_{1}^{\prime}} \\
& +\sum_{k_{1}^{\prime}, k_{1}} h_{k}(t) F_{k^{\prime}, k, k_{1}, k_{1}^{\prime}}^{*}(t) n_{k}: c_{k_{1}^{\prime} \downarrow}^{\dagger} c_{k_{1} \downarrow}: \delta_{k^{\prime}+k_{1}, k+k_{1}^{\prime}} \\
& + \text { "irrelevant terms" } \\
& +O\left(U^{2}\right) .
\end{aligned}
$$

where the "irrelevant terms" do not have an operator structure proportional to $: c_{k^{\prime} \downarrow}^{\dagger} c_{k \downarrow}:$ or 1 . Actually, only the olive terms in the equation above contribute, because the black terms drop out when calculating the imaginary part in eq. 6.9. As much as the number of terms has decreased, we omit the color coding for the calculation of the antiparallel spin susceptibility 
from now on. Then, we have

$$
\begin{aligned}
\chi_{x^{\prime}, x}^{\uparrow \downarrow}(t)= & \frac{2}{\Omega^{2}} \sum_{k^{\prime}, k, q^{\prime}, q} \mathfrak{I}\left(e^{i\left(k^{\prime}-k\right) x^{\prime}} e^{i\left(q^{\prime}-q\right) x}\left\langle\boldsymbol{c}_{k^{\prime} \uparrow}^{\dagger}(t) \boldsymbol{c}_{k \uparrow}(t) \boldsymbol{c}_{q^{\prime} \downarrow}^{\dagger}(0) \boldsymbol{c}_{q \downarrow}(0)\right\rangle\right) \\
= & \frac{2}{\Omega^{2}} \sum_{k^{\prime}, k, q^{\prime}, q} \mathfrak{J}\left(e^{i\left(k^{\prime}-k\right)\left(x^{\prime}-x\right)} h_{k^{\prime}}^{*}(t) F_{k, k^{\prime}, q, q^{\prime}}(t) n_{k^{\prime}}\left(1-n_{q^{\prime}}\right) n_{q^{\prime}} \delta_{k^{\prime}+q^{\prime}, k+q}\right) \\
& +\frac{2}{\Omega^{2}} \sum_{k^{\prime}, k, q^{\prime}, q} \mathfrak{J}\left(e^{i\left(k^{\prime}-k\right)\left(x^{\prime}-x\right)} h_{k}(t) F_{k^{\prime}, k, q^{\prime}, q}^{*}(t) n_{k}\left(1-n_{q^{\prime}}\right) n_{q^{\prime}} \delta_{k^{\prime}+q^{\prime}, k+q}\right) \\
& +O\left(U^{2}\right) .
\end{aligned}
$$

Finally, we can insert the solutions for $h_{k}(t)$ and $F_{k, k_{1}, k_{2}^{\prime}, k_{2}}(t)$ from eqs. 5.56 and 5.57) to get

$$
\begin{aligned}
\chi_{x^{\prime}, x}^{\uparrow \downarrow}(t)= & -i \frac{U}{\Omega^{3}} \sum_{k^{\prime}, k} e^{i\left(k^{\prime}-k\right)\left(x^{\prime}-x\right)} e^{i\left(\epsilon_{k^{\prime}}-\epsilon_{k}\right) t}\left(n_{k^{\prime}}-n_{k}\right) \sum_{q^{\prime}, q} \frac{1-e^{-i\left(\Delta \epsilon_{k^{\prime}, k, q^{\prime}, q}\right) t}}{\Delta \epsilon_{k^{\prime}, k, q^{\prime}, q}}\left(n_{q^{\prime}}-n_{q}\right) \delta_{k^{\prime}+q^{\prime}, k+q} \\
& +O\left(U^{2}\right) .
\end{aligned}
$$

\subsubsection{For parallel spins}

For parallel spins, there will be no contribution to the susceptibility of first order in $U$. Therefore, we consider also the second order terms from eq. 6.7). However, only the black and red terms completely contract with $c_{q^{\prime} \uparrow}^{\dagger}(0) c_{q \uparrow}(0)$ from eq. 6.10), i.e., we only use

$$
\begin{aligned}
c_{k^{\prime} \uparrow}^{\dagger}(t) \boldsymbol{c}_{k \uparrow}(t)= & \left|h_{k^{\prime}}(t)\right|^{2} n_{k^{\prime}} \delta_{k^{\prime}, k}+\sum_{k_{1}, k_{2}^{\prime}, k_{2}}\left|F_{k^{\prime}, k_{1}, k_{2}^{\prime}, k_{2}}(t)\right|^{2} n_{k_{1}}\left(1-n_{k_{2}^{\prime}}\right) n_{k_{2}} \delta_{k^{\prime}+k_{2}^{\prime}, k_{1}+k_{2}} \delta_{k^{\prime}, k} \\
& +h_{k^{\prime}}^{*}(t) h_{k}(t): c_{k^{\prime} \uparrow}^{\dagger} c_{k \uparrow}: \\
& +\sum_{k_{i^{\prime}, k_{i}}} F_{k^{\prime}, k_{1}^{\prime}, k_{2}^{\prime}, k_{2}}(t) F_{k, k_{1}, k_{2}^{\prime}, k_{2}}(t)\left(1-n_{k_{2}^{\prime}}\right) n_{k_{2}}: c_{k_{1}^{\prime} \uparrow}^{\dagger} c_{k_{1} \uparrow}: \delta_{k_{k}+k_{2}^{\prime}, k_{1}+k_{2}} \delta_{k^{\prime}+k_{1}, k+k_{1}^{\prime}} \\
& +\sum_{k_{1}^{\prime}, k_{1}} h_{k^{\prime}}^{*}(t)\left(G_{k, k^{\prime}, k_{1}^{\prime}, k_{1}}(t)-G_{k_{,}, k_{1}, k_{1}^{\prime}, k^{\prime}}(t)\right) n_{k^{\prime}}: c_{k_{1}^{\prime} \uparrow}^{\dagger} c_{k_{1} \uparrow}: \delta_{k^{\prime}+k_{1}, k+k_{1}^{\prime}} \\
& +\sum_{k_{1}^{\prime}, k_{1}} h_{k}(t)\left(G_{k^{\prime}, k, k_{1}, k_{1}^{\prime}}^{*}(t)-G_{k^{\prime}, k_{1}^{\prime}, k_{1}, k}^{*}(t)\right) n_{k}: c_{k_{1}^{\prime} \uparrow}^{\dagger} c_{k_{1} \uparrow}: \delta_{k^{\prime}+k_{1}, k+k_{1}^{\prime}} \\
& + \text { "irrelevant terms" } \\
& +O\left(U^{3}\right) .
\end{aligned}
$$


The black terms drop out again for the same reason like above. We waive the color coding again and get

$$
\begin{aligned}
\chi_{x^{\prime}, x}^{\uparrow \uparrow}(t)= & \frac{2}{\Omega^{2}} \sum_{k^{\prime}, k, q^{\prime}, q} \mathfrak{I}\left(e^{i\left(k^{\prime}-k\right) x^{\prime}} e^{i\left(q^{\prime}-q\right) x}\left\langle c_{k^{\prime} \uparrow}^{\dagger}(t) \boldsymbol{c}_{k \uparrow}(t) \boldsymbol{c}_{q^{\prime} \uparrow}^{\dagger}(0) \boldsymbol{c}_{q \uparrow}(0)\right\rangle\right) \\
= & -i \frac{1}{\Omega^{2}} \sum_{k^{\prime}, k} e^{i\left(k^{\prime}-k\right)\left(x^{\prime}-x\right)}\left(n_{k^{\prime}}-n_{k}\right) h_{k^{\prime}}^{*}(t) h_{k}(t) \\
& -i \frac{1}{\Omega^{2}} \sum_{k^{\prime}, k} e^{i\left(k^{\prime}-k\right)\left(x^{\prime}-x\right)}\left(n_{k^{\prime}}-n_{k}\right) \\
& +\frac{2}{\Omega^{2}} \sum_{k^{\prime}, k} e^{i\left(k^{\prime}-k\right)\left(x^{\prime}-x\right)}\left(n_{k^{\prime}}-n_{k}\right) \\
& \times \sum_{k_{1}^{\prime}, k_{i}^{\prime}, k_{1}} \mathfrak{J}\left(h_{k_{1}^{\prime}}^{*}(t) G_{k_{1}, k_{1}^{\prime}, k^{\prime}, k}(t)\right) n_{k_{1}^{\prime}} \delta_{k^{\prime}+k_{1}, k+k_{1}^{\prime}} \\
& -\frac{2}{\Omega^{2}} \sum_{k^{\prime}, k} e^{i\left(k^{\prime}-k\right)\left(x^{\prime}-x\right)}\left(n_{k^{\prime}}-n_{k}\right) \\
& \times \sum_{k_{1}^{\prime}, k_{1}, k, k_{2}, k_{2}^{\prime}}(t) n_{k_{2}^{\prime}}\left(1-n_{k_{2}}\right) \delta_{k+k_{2}^{\prime}, k_{1}+k_{2}} \delta_{k^{\prime}+k_{1}, k+k_{1}^{\prime}} \\
& +O\left(h_{k_{1}^{\prime}}^{*}(t) G_{k_{1}, k, k^{\prime}, k_{1}^{\prime}}(t)\right) n_{k_{1}^{\prime}} \delta_{k^{\prime}+k_{1}, k+k_{1}^{\prime}}
\end{aligned}
$$


Next, we insert the solutions for the coefficients from eqs. (5.56) - 5.58), which yields

$$
\begin{aligned}
& \chi_{x^{\prime}, x}^{\uparrow \uparrow}(t)=-i \frac{1}{\Omega^{2}} \sum_{k^{\prime}, k} e^{i\left(k^{\prime}-k\right)\left(x^{\prime}-x\right)} e^{i\left(\tilde{\epsilon}_{k^{\prime}}-\tilde{\epsilon}_{k}\right) t}\left(n_{k^{\prime}}-n_{k}\right) \\
& +i \frac{2 U^{2}}{\Omega^{4}} \sum_{k^{\prime}, k} e^{i\left(k^{\prime}-k\right)\left(x^{\prime}-x\right)} e^{i\left(\epsilon_{k^{\prime}}-\epsilon_{k}\right) t}\left(n_{k^{\prime}}-n_{k}\right) \\
& \times \sum_{k_{1}, k_{2}^{\prime}, k_{2}} \frac{1-e^{i\left(\Delta \epsilon_{k, k_{1}, k_{2}^{\prime}, k_{2}}\right) t}}{\left(\Delta \epsilon_{k, k_{1}, k_{2}^{\prime}, k_{2}}\right)^{2}} \\
& \times\left(\left(1-n_{k_{1}}\right) n_{k_{2}^{\prime}}\left(1-n_{k_{2}}\right)+n_{k_{1}}\left(1-n_{k_{2}^{\prime}}\right) n_{k_{2}}\right) \delta_{k+k_{2}^{\prime}, k_{1}+k_{2}} \\
& -i \frac{U^{2}}{\Omega^{4}} \sum_{k^{\prime}, k} e^{i\left(k^{\prime}-k\right)\left(x^{\prime}-x\right)} e^{i\left(\epsilon_{k^{\prime}}-\epsilon_{k}\right) t}\left(n_{k^{\prime}}-n_{k}\right) \\
& \times \sum_{k_{i}^{\prime}, k_{i}} \frac{1-e^{-i\left(\Delta \epsilon_{k^{\prime}, k_{1}^{\prime}, k_{2}^{\prime}, k_{2}}\right) t}-e^{i\left(\Delta \epsilon_{k, k_{1}, k_{2}^{\prime}, k_{2}}\right) t}+e^{-i\left(\Delta \epsilon_{k^{\prime}, k, k_{1}, k_{1}}\right) t}}{\left(\Delta \epsilon_{k^{\prime}, k_{1}^{\prime}, k_{2}^{\prime}, k_{2}}\right)\left(\Delta \epsilon_{k, k_{1}, k_{2}^{\prime}, k_{2}}\right)} \\
& \times n_{k_{2}^{\prime}}\left(1-n_{k_{2}}\right) \delta_{k+k_{2}^{\prime}, k_{1}+k_{2}} \delta_{k^{\prime}+k_{1}, k+k_{1}^{\prime}} \\
& -i \frac{U^{2}}{\Omega^{4}} \sum_{k^{\prime}, k} e^{i\left(k^{\prime}-k\right)\left(x^{\prime}-x\right)} e^{i\left(\epsilon_{k^{\prime}}-\epsilon_{k}\right) t}\left(n_{k^{\prime}}-n_{k}\right) \\
& \times \sum_{k_{i}^{\prime}, k_{i}} \frac{\Delta \epsilon_{k^{\prime}, k, k_{2}, k_{2}^{\prime}}}{\Delta \epsilon_{k_{1}^{\prime}, k_{1}, k_{2}, k_{2}^{\prime}}}\left(\frac{1-e^{-i\left(\Delta \epsilon_{k^{\prime}, k, k_{1}, k_{1}^{\prime}}\right) t}}{\left(\Delta \epsilon_{k^{\prime}, k, k_{2}, k_{2}^{\prime}}\right)^{2}+\left(\Delta \epsilon_{k_{1}^{\prime}, k_{1}, k_{2}, k_{2}^{\prime}}\right)^{2}}-\frac{1-e^{-i\left(\Delta \epsilon_{\left.k^{\prime}, k, k_{2}, k_{2}^{\prime}\right) t}\right.}}{\left(\Delta \epsilon_{k^{\prime}, k, k_{2}, k_{2}^{\prime}}\right)^{2}}\right) \\
& \times\left(n_{k_{1}^{\prime}}-n_{k_{1}}\right)\left(n_{k_{2}^{\prime}}-n_{k_{2}}\right) \delta_{k^{\prime}+k_{2}, k+k_{2}^{\prime}} \delta_{k^{\prime}+k_{1}, k+k_{1}^{\prime}} \\
& -i \frac{U^{2}}{\Omega^{4}} \sum_{k^{\prime}, k} e^{i\left(k^{\prime}-k\right)\left(x^{\prime}-x\right)} e^{i\left(\epsilon_{k^{\prime}}-\epsilon_{k}\right) t}\left(n_{k^{\prime}}-n_{k}\right) \\
& \times \sum_{k_{i}^{\prime}, k_{i}} \frac{\Delta \epsilon_{k^{\prime}, k_{1}^{\prime}, k_{2}^{\prime}, k_{2}}}{\Delta \epsilon_{k, k_{1}, k_{2}^{\prime}, k_{2}}}\left(\frac{1-e^{-i\left(\Delta \epsilon_{k^{\prime}, k, k_{1}, k_{1}^{\prime}}\right) t}}{\left(\Delta \epsilon_{k^{\prime}, k_{1}^{\prime}, k_{2}^{\prime}, k_{2}}\right)^{2}+\left(\Delta \epsilon_{k, k_{1}, k_{2}^{\prime}, k_{2}}\right)^{2}}-\frac{1-e^{-i\left(\Delta \epsilon_{k^{\prime}, k_{1}^{\prime}, k_{2}^{\prime}, k_{2}}\right) t}}{\left(\Delta \epsilon_{k^{\prime}, k_{1}^{\prime}, k_{2}^{\prime}, k_{2}}\right)^{2}}\right) \\
& \times n_{k_{1}^{\prime}}\left(n_{k_{2}^{\prime}}-n_{k_{2}}\right) \delta_{k+k_{2}^{\prime}, k_{1}+k_{2}} \delta_{k^{\prime}+k_{1}, k+k_{1}^{\prime}} \\
& -i \frac{U^{2}}{\Omega^{4}} \sum_{k^{\prime}, k} e^{i\left(k^{\prime}-k\right)\left(x^{\prime}-x\right)} e^{i\left(\epsilon_{k^{\prime}}-\epsilon_{k}\right) t}\left(n_{k^{\prime}}-n_{k}\right) \\
& \times \sum_{k_{i}^{\prime}, k_{i}} \frac{\Delta \epsilon_{k, k_{1}, k_{2}^{\prime}, k_{2}}}{\Delta \epsilon_{k^{\prime}, k_{1}^{\prime}, k_{2}^{\prime}, k_{2}}}\left(\frac{1-e^{-i\left(\Delta \epsilon_{k^{\prime}, k, k_{1}, k_{1}^{\prime}}\right) t}}{\left(\Delta \epsilon_{k^{\prime}, k_{1}^{\prime}, k_{2}^{\prime}, k_{2}}\right)^{2}+\left(\Delta \epsilon_{k, k_{1}, k_{2}^{\prime}, k_{2}}\right)^{2}}-\frac{1-e^{i\left(\Delta \epsilon_{k, k_{1}, k_{2}^{\prime}, k_{2}}\right) t}}{\left(\Delta \epsilon_{k, k_{1}, k_{2}^{\prime}, k_{2}}\right)^{2}}\right) \\
& \times n_{k_{1}}\left(n_{k_{2}^{\prime}}-n_{k_{2}}\right) \delta_{k+k_{2}^{\prime}, k_{1}+k_{2}} \delta_{k^{\prime}+k_{1}, k+k_{1}^{\prime}}
\end{aligned}
$$




$$
\begin{aligned}
& -i \frac{U^{2}}{\Omega^{4}} \sum_{k^{\prime}, k} e^{i\left(k^{\prime}-k\right)\left(x^{\prime}-x\right)} e^{i\left(\epsilon_{k^{\prime}}-\epsilon_{k}\right) t}\left(n_{k^{\prime}}-n_{k}\right) \\
& \times \sum_{k_{i}^{\prime}, k_{i}} \frac{1-e^{i\left(\Delta \epsilon_{k, k^{\prime}, k_{1}^{\prime}, k_{1}}\right) t}}{\left(\Delta \epsilon_{k_{1}^{\prime}, k_{1}, k_{2}^{\prime}, k_{2}}\right)^{2}+\left(\Delta \epsilon_{k^{\prime}, k, k_{2}^{\prime}, k_{2}}\right)^{2}} n_{k_{1}^{\prime}}\left(n_{k_{2}^{\prime}}-n_{k_{2}}\right) \delta_{k_{1}^{\prime}+k_{2}^{\prime}, k_{1}+k_{2}} \delta_{k^{\prime}+k_{1}, k+k_{1}^{\prime}} \\
& +i \frac{U^{2}}{\Omega^{4}} \sum_{k^{\prime}, k} e^{i\left(k^{\prime}-k\right)\left(x^{\prime}-x\right)} e^{i\left(\epsilon_{k^{\prime}}-\epsilon_{k}\right) t}\left(n_{k^{\prime}}-n_{k}\right) \\
& \times \sum_{k_{i}^{\prime}, k_{i}} \frac{\left(\Delta \epsilon_{k_{1}, k, k^{\prime}, k_{1}^{\prime}}\right)\left(\Delta \epsilon_{k_{1}, k, k_{2}^{\prime}, k_{2}}\right)}{\left(\Delta \epsilon_{k_{1}, k, k_{2}^{\prime}, k_{2}}\right)^{2}+\left(\Delta \epsilon_{k^{\prime}, k_{1}^{\prime}, k_{2}, k_{2}^{\prime}}\right)^{2}-\left(\Delta \epsilon_{k_{1}, k, k^{\prime}, k_{1}^{\prime}}\right)^{2}} \\
& \times\left(\frac{1-e^{i\left(\Delta \epsilon_{k, k^{\prime}, k_{1}^{\prime}, k_{1}}\right) t}}{\left(\Delta \epsilon_{k_{1}, k, k_{2}^{\prime}, k_{2}}\right)^{2}+\left(\Delta \epsilon_{k^{\prime}, k_{1}^{\prime}, k_{2}, k_{2}^{\prime}}\right)^{2}}-\frac{e^{-i \epsilon_{k_{1}^{\prime}} t}-e^{-i\left(\epsilon_{k_{1}}-\epsilon_{k}+\epsilon_{k^{\prime}}\right) t}}{\left(\Delta \epsilon_{k_{1}, k, k^{\prime}, k_{1}^{\prime}}\right)^{2}}\right) \\
& \times n_{k_{1}^{\prime}}\left(n_{k_{2}^{\prime}}-n_{k_{2}}\right) \delta_{k_{2}^{\prime}+k_{1}^{\prime}, k_{2}+k^{\prime}} \delta_{k^{\prime}+k_{1}, k+k_{1}^{\prime}} \\
& -i \frac{U^{2}}{\Omega^{4}} \sum_{k^{\prime}, k} e^{i\left(k^{\prime}-k\right)\left(x^{\prime}-x\right)} e^{i\left(\epsilon_{k^{\prime}}-\epsilon_{k}\right) t}\left(n_{k^{\prime}}-n_{k}\right) \\
& \times \sum_{k_{i}^{\prime}, k_{i}} \frac{\left(\Delta \epsilon_{k_{1}^{\prime}, k^{\prime}, k, k_{1}}\right)\left(\Delta \epsilon_{k_{1}^{\prime}, k^{\prime}, k_{2}, k_{2}^{\prime}}\right)}{\left(\Delta \epsilon_{k_{1}^{\prime}, k^{\prime}, k_{2}, k_{2}^{\prime}}\right)^{2}+\left(\Delta \epsilon_{k, k_{1}, k_{2}^{\prime}, k_{2}}\right)^{2}-\left(\Delta \epsilon_{k_{1}, k, k^{\prime}, k_{1}^{\prime}}\right)^{2}} \\
& \times\left(\frac{1-e^{-i\left(\Delta \epsilon_{k^{\prime}, k, k_{1}, k_{1}^{\prime}}\right) t}}{\left(\Delta \epsilon_{k_{1}^{\prime}, k^{\prime}, k_{2}, k_{2}^{\prime}}\right)^{2}+\left(\Delta \epsilon_{k, k_{1}, k_{2}^{\prime}, k_{2}}\right)^{2}}-\frac{e^{i \epsilon_{k_{1}} t}-e^{i\left(\epsilon_{k}-\epsilon_{k^{\prime}}+\epsilon_{k_{1}^{\prime}}\right) t}}{\left(\Delta \epsilon_{k_{1}, k, k^{\prime}, k_{1}^{\prime}}\right)^{2}}\right) \\
& \times n_{k_{1}}\left(n_{k_{2}^{\prime}}-n_{k_{2}}\right) \delta_{k_{2}+k_{1}, k_{2}^{\prime}+k} \delta_{k^{\prime}+k_{1}, k+k_{1}^{\prime}} \\
& +i \frac{U^{2}}{\Omega^{4}} \sum_{k^{\prime}, k} e^{i\left(k^{\prime}-k\right)\left(x^{\prime}-x\right)} e^{i\left(\epsilon_{k^{\prime}}-\epsilon_{k}\right) t}\left(n_{k^{\prime}}-n_{k}\right) \\
& \times \sum_{k_{i}^{\prime}, k_{i}} \frac{\left(\Delta \epsilon_{k_{1}, k, k^{\prime}, k_{1}^{\prime}}\right)\left(\Delta \epsilon_{k^{\prime}, k_{1}^{\prime}, k_{2}^{\prime}, k_{2}}\right)}{\left(\Delta \epsilon_{k^{\prime}, k_{1}^{\prime}, k_{2}^{\prime}, k_{2}}\right)^{2}+\left(\Delta \epsilon_{k_{1}, k, k_{2}, k_{2}^{\prime}}\right)^{2}-\left(\Delta \epsilon_{k_{1}, k, k^{\prime}, k_{1}^{\prime}}\right)^{2}} \\
& \times\left(\frac{1-e^{-i\left(\Delta \epsilon_{k^{\prime}, k, k_{1}, k_{1}^{\prime}}\right) t}}{\left(\Delta \epsilon_{k^{\prime}, k_{1}^{\prime}, k_{2}^{\prime}, k_{2}}\right)^{2}+\left(\Delta \epsilon_{k_{1}, k, k_{2}, k_{2}^{\prime}}\right)^{2}}-\frac{e^{-i \epsilon_{k_{1}^{\prime}} t}-e^{-i\left(\epsilon_{k^{\prime}}-\epsilon_{k}+\epsilon_{k_{1}}\right) t}}{\left(\Delta \epsilon_{k_{1}, k, k^{\prime}, k_{1}^{\prime}}\right)^{2}}\right) \\
& \times n_{k_{1}^{\prime}}\left(n_{k_{2}^{\prime}}-n_{k_{2}}\right) \delta_{k_{2}^{\prime}+k, k_{2}+k_{1}} \delta_{k^{\prime}+k_{1}, k+k_{1}^{\prime}} \\
& +i \frac{U^{2}}{\Omega^{4}} \sum_{k^{\prime}, k} e^{i\left(k^{\prime}-k\right)\left(x^{\prime}-x\right)} e^{i\left(\epsilon_{k^{\prime}}-\epsilon_{k}\right) t}\left(n_{k^{\prime}}-n_{k}\right) \\
& \times \sum_{k_{i}^{\prime}, k_{i}} \frac{\left(\Delta \epsilon_{k_{1}, k, k^{\prime}, k_{1}^{\prime}}\right)\left(\Delta \epsilon_{k, k_{1}, k_{2}, k_{2}^{\prime}}\right)}{\left(\Delta \epsilon_{k, k_{1}, k_{2}, k_{2}^{\prime}}\right)^{2}+\left(\Delta \epsilon_{k_{1}^{\prime}, k^{\prime}, k_{2}^{\prime}, k_{2}}\right)^{2}-\left(\Delta \epsilon_{k_{1}, k, k^{\prime}, k_{1}^{\prime}}\right)^{2}} \\
& \times\left(\frac{1-e^{-i\left(\Delta \epsilon_{k^{\prime}, k, k_{1}, k_{1}^{\prime}}\right) t}}{\left(\Delta \epsilon_{k, k_{1}, k_{2}, k_{2}^{\prime}}\right)^{2}+\left(\Delta \epsilon_{k_{1}^{\prime}, k^{\prime}, k_{2}^{\prime}, k_{2}}\right)^{2}}-\frac{e^{i \epsilon_{k_{1}} t}-e^{i\left(\epsilon_{k_{1}^{\prime}}-\epsilon_{k^{\prime}}+\epsilon_{k}\right) t}}{\left(\Delta \epsilon_{k_{1}, k, k^{\prime}, k_{1}^{\prime}}\right)^{2}}\right) \\
& \times n_{k_{1}}\left(n_{k_{2}^{\prime}}-n_{k_{2}}\right) \delta_{k_{2}+k^{\prime}, k_{2}^{\prime}+k_{1}^{\prime}} \delta_{k^{\prime}+k_{1}, k+k_{1}^{\prime}} \\
& +O\left(U^{3}\right) .
\end{aligned}
$$




\subsection{Equal-time correlation function}

For the calculation of the equal-time connected density-density correlation function from eq. 6.3), all terms from eq. 6.7) contribute. We need to calculate all full contractions in

$$
\begin{aligned}
C_{x^{\prime}, x}^{\sigma^{\prime} \sigma}(t)= & \left\langle\boldsymbol{n}_{x^{\prime}, \sigma^{\prime}}(t) \boldsymbol{n}_{x, \sigma}(t)\right\rangle-\left\langle\boldsymbol{n}_{x^{\prime}, \sigma^{\prime}}(t)\right\rangle\left\langle\boldsymbol{n}_{x, \sigma}(t)\right\rangle \\
= & \frac{1}{\Omega^{2}} \sum_{k^{\prime}, k, q^{\prime}, q} e^{i\left(k^{\prime}-k\right) x^{\prime}} e^{i\left(q^{\prime}-q\right) x}\left\langle\boldsymbol{c}_{k^{\prime} \sigma^{\prime}}^{\dagger}(t) \boldsymbol{c}_{k \sigma^{\prime}}(t) \boldsymbol{c}_{q^{\prime} \sigma}^{\dagger}(t) \boldsymbol{c}_{q \sigma}(t)\right\rangle \\
& -\frac{1}{\Omega^{2}} \sum_{k^{\prime}, k, q^{\prime}, q} e^{i\left(k^{\prime}-k\right) x^{\prime}} e^{i\left(q^{\prime}-q\right) x}\left\langle\boldsymbol{c}_{k^{\prime} \sigma^{\prime}}^{\dagger}(t) \boldsymbol{c}_{k \sigma^{\prime}}(t)\right\rangle\left\langle\boldsymbol{c}_{q^{\prime} \sigma}^{\dagger}(t) \boldsymbol{c}_{q \sigma}(t)\right\rangle .
\end{aligned}
$$

The substraction of $\left\langle\boldsymbol{n}_{x^{\prime}, \sigma^{\prime}}(t)\right\rangle\left\langle\boldsymbol{n}_{x, \sigma}(t)\right\rangle$ spares us the black terms in eq. 6.7).

\subsubsection{For antiparallel spins}

For antiparallel spins, the equal-time correlation function has a first-order contribution in $U$. Therefore, we neglect the second-order terms in eq. (6.7). The orange terms only completely contract among each other, which would be of second order, hence they are irrelevant. We only need

$$
\begin{aligned}
\boldsymbol{c}_{k^{\prime} \uparrow}^{\dagger}(t) \boldsymbol{c}_{k \uparrow}(t)= & h_{k^{\prime}}^{*}(t) h_{k}(t): c_{k^{\prime} \uparrow}^{\dagger} c_{k \uparrow}: \\
& +\sum_{k_{1}^{\prime}, k_{1}} h_{k^{\prime}}^{*}(t) F_{k_{,}, k^{\prime}, k_{1}^{\prime}, k_{1}}(t) n_{k^{\prime}}: c_{k_{1}^{\prime} \downarrow}^{\dagger} c_{k_{1} \downarrow}: \delta_{k^{\prime}+k_{1}, k+k_{1}^{\prime}} \\
& +\sum_{k_{1}^{\prime}, k_{1}} h_{k^{\prime}}(t) F_{k^{\prime}, k^{\prime}, k_{1}, k_{1}^{\prime}}^{*}(t) n_{k}: c_{k_{1}^{\prime} \downarrow}^{\dagger} c_{k_{1} \downarrow}: \delta_{k^{\prime}+k_{1}, k_{k}+k_{1}^{\prime}} \\
& + \text { "irrelevant terms" } \\
& +O\left(U^{2}\right) .
\end{aligned}
$$

We omit the color coding and calcuclate the contractions between the above equation and its spin-down counterpart, yielding

$$
\begin{aligned}
C_{x^{\prime}, x}^{\uparrow \downarrow}(t)= & \frac{1}{\Omega^{2}} \sum_{k^{\prime}, k, q^{\prime}, q} e^{i\left(k^{\prime}-k\right)\left(x^{\prime}-x\right)} h_{k^{\prime}}^{*}(t) h_{k}(t) h_{q^{\prime}}^{*}(t) F_{q, q^{\prime}, k, k^{\prime}}(t) n_{k^{\prime}}\left(1-n_{k}\right) n_{q^{\prime}} \delta_{k^{\prime}+q^{\prime}, k+q} \\
& +\frac{1}{\Omega^{2}} \sum_{k^{\prime}, k, q^{\prime}, q} e^{i\left(k^{\prime}-k\right)\left(x^{\prime}-x\right)} h_{k^{\prime}}^{*}(t) h_{k}(t) h_{q}(t) F_{q^{\prime}, q, k^{\prime}, k}^{*}(t) n_{k^{\prime}}\left(1-n_{k}\right) n_{q^{\prime}} \delta_{k^{\prime}+q^{\prime}, k+q} \\
& +\frac{1}{\Omega^{2}} \sum_{k^{\prime}, k, q^{\prime}, q} e^{i\left(k^{\prime}-k\right)\left(x^{\prime}-x\right)} h_{k^{\prime}}(t) h_{k}^{*}(t) h_{q}^{*}(t) F_{q^{\prime}, q, k^{\prime}, k}(t) n_{k^{\prime}}\left(1-n_{k}\right) n_{q} \delta_{k^{\prime}+q^{\prime}, k+q} \\
& +\frac{1}{\Omega^{2}} \sum_{k^{\prime}, k, q^{\prime}, q} e^{i\left(k^{\prime}-k\right)\left(x^{\prime}-x\right)} h_{k^{\prime}}(t) h_{k}^{*}(t) h_{q^{\prime}}(t) F_{q, q^{\prime}, k, k^{\prime}}^{*}(t) n_{k^{\prime}}\left(1-n_{k}\right) n_{q^{\prime}} \delta_{k^{\prime}+q^{\prime}, k+q} \\
& +O\left(U^{2}\right) .
\end{aligned}
$$


When we insert the coefficients from eqs. 5.56) and (5.57), we arrive at

$$
\begin{aligned}
C_{x^{\prime}, x}^{\uparrow \downarrow}(t)= & \frac{U}{\Omega^{3}} \sum_{k^{\prime}, k, q^{\prime}, q} e^{i\left(k^{\prime}-k\right)\left(x^{\prime}-x\right)} \frac{1-e^{i\left(\Delta \epsilon_{k^{\prime}, k, q^{\prime}, q}\right) t}}{\Delta \epsilon_{k^{\prime}, k, q^{\prime}, q}} n_{k^{\prime}}\left(1-n_{k}\right)\left(n_{q^{\prime}}-n_{q}\right) \delta_{k^{\prime}+q^{\prime}, k+q} \\
& +\frac{U}{\Omega^{3}} \sum_{k^{\prime}, k, q^{\prime}, q} e^{i\left(k^{\prime}-k\right)\left(x^{\prime}-x\right)} \frac{1-e^{-i\left(\Delta \epsilon_{k^{\prime}, k, q^{\prime}, q}\right) t}}{\Delta \epsilon_{k^{\prime}, k, q^{\prime}, q}} n_{k^{\prime}}\left(1-n_{k}\right)\left(n_{q^{\prime}}-n_{q}\right) \delta_{k^{\prime}+q^{\prime}, k+q} \\
& +O\left(U^{2}\right) .
\end{aligned}
$$

Now, let us interchange the summation indices, $\left(k^{\prime}, k\right) \leftrightarrow\left(q, q^{\prime}\right)$, and use that $q^{\prime}-q=k-k^{\prime}$ due to the $\delta$-function. Then, the expression above changes to

$$
\begin{aligned}
C_{x^{\prime}, x}^{\uparrow \downarrow}(t)= & \frac{U}{\Omega^{3}} \sum_{k^{\prime}, k} e^{i\left(k^{\prime}-k\right)\left(x^{\prime}-x\right)}\left(n_{k^{\prime}}-n_{k}\right) \sum_{q^{\prime}, q} \frac{1-e^{-i\left(\Delta \epsilon_{k^{\prime}, k, q^{\prime}, q}\right) t}}{\Delta \epsilon_{k^{\prime}, k, q^{\prime}, q}}\left(1-n_{q^{\prime}}\right) n_{q} \delta_{k^{\prime}+q^{\prime}, k+q} \\
& +\frac{U}{\Omega^{3}} \sum_{k^{\prime}, k} e^{i\left(k^{\prime}-k\right)\left(x^{\prime}-x\right)}\left(n_{k^{\prime}}-n_{k}\right) \sum_{q^{\prime}, q} \frac{1-e^{i\left(\Delta \epsilon_{k^{\prime}, k, q^{\prime}, q}\right) t}}{\Delta \epsilon_{k^{\prime}, k, q^{\prime}, q}}\left(1-n_{q^{\prime}}\right) n_{q} \delta_{k^{\prime}+q^{\prime}, k+q} \\
& +O\left(U^{2}\right) .
\end{aligned}
$$

Finally, this can be written as

$$
\begin{aligned}
C_{x^{\prime}, x}^{\uparrow \downarrow}(t)= & \frac{2 U}{\Omega^{3}} \sum_{k^{\prime}, k} e^{i\left(k^{\prime}-k\right)\left(x^{\prime}-x\right)}\left(n_{k^{\prime}}-n_{k}\right) \sum_{q^{\prime}, q} \frac{1-\cos \left(\left(\Delta \epsilon_{k^{\prime}, k, q^{\prime}, q}\right) t\right)}{\Delta \epsilon_{k^{\prime}, k, q^{\prime}, q}}\left(1-n_{q^{\prime}}\right) n_{q} \delta_{k^{\prime}+q^{\prime}, k+q} \\
& +O\left(U^{2}\right)
\end{aligned}
$$

\subsubsection{For parallel spins}

For parallel spins, the equal-time connected correlation function is of second order in $U$ and hence all terms in eq. (6.7) are relevant, except for the black ones. Calculating all full contractions, we find that

$$
\begin{aligned}
C_{x^{\prime}, x}^{\uparrow \uparrow}(t)= & \frac{1}{\Omega^{2}} \sum_{k^{\prime}, k} e^{i\left(k^{\prime}-k\right)\left(x^{\prime}-x\right)} n_{k^{\prime}}\left(1-n_{k}\right)\left|h_{k^{\prime}}(t)\right|^{2}\left|h_{k}(t)\right|^{2} \\
& +\frac{2}{\Omega^{2}} \sum_{k^{\prime}, k} e^{i\left(k^{\prime}-k\right)\left(x^{\prime}-x\right)} n_{k^{\prime}}\left(1-n_{k}\right) \\
& \quad \times \sum_{k_{i}^{\prime}, k_{i}} R\left(h_{k^{\prime}}^{*}(t) h_{k}(t) F_{k_{1}^{\prime}, k^{\prime}, k_{2}^{\prime}, k_{2}}(t) F_{k_{1}, k, k_{2}^{\prime}, k_{2}}^{*}(t)\right)\left(1-n_{k_{2}^{\prime}}\right) n_{k_{2}} \delta_{k_{1}^{\prime}+k_{2}^{\prime}, k^{\prime}+k_{2}} \delta_{k^{\prime}+k_{1}, k+k_{1}^{\prime}} \\
& +\frac{2}{\Omega^{2}} \sum_{k^{\prime}, k} e^{i\left(k^{\prime}-k\right)\left(x^{\prime}-x\right)} n_{k^{\prime}}\left(1-n_{k}\right) \\
& \quad \times \sum_{k_{1}^{\prime}, k_{1}} R\left(h_{k^{\prime}}^{*}(t) h_{k}(t) h_{k_{1}^{\prime}}(t)\left(G_{k_{1}, k_{1}^{\prime}, k^{\prime}, k}^{*}(t)-G_{k_{1}, k, k^{\prime}, k_{1}^{\prime}}^{*}(t)\right)\right) n_{k_{1}^{\prime}} \delta_{k^{\prime}+k_{1}, k+k_{1}^{\prime}}
\end{aligned}
$$




$$
\begin{aligned}
& +\frac{2}{\Omega^{2}} \sum_{k^{\prime}, k} e^{i\left(k^{\prime}-k\right)\left(x^{\prime}-x\right)} n_{k^{\prime}}\left(1-n_{k}\right) \\
& \times \sum_{k_{1}^{\prime}, k_{1}} \mathfrak{R}\left(h_{k^{\prime}}^{*}(t) h_{k}(t) h_{k_{1}}^{*}(t)\left(G_{k_{1}^{\prime}, k_{1}, k, k^{\prime}}(t)-G_{k_{1}^{\prime}, k^{\prime}, k, k_{1}}(t)\right)\right) n_{k_{1}} \delta_{k^{\prime}+k_{1}, k+k_{1}^{\prime}} \\
& +\frac{1}{\Omega^{2}} \sum_{k^{\prime}, k} e^{i\left(k^{\prime}-k\right)\left(x^{\prime}-x\right)} n_{k^{\prime}}\left(1-n_{k}\right) \\
& \times \sum_{k_{i}^{\prime}, k_{i}} h_{k_{1}^{\prime}}^{*}(t) F_{k_{1}, k_{1}^{\prime}, k^{\prime}, k}(t) h_{k_{2}^{\prime}}^{*}(t) F_{k_{2}, k_{2}^{\prime}, k^{\prime}, k^{\prime}}(t) n_{k_{1}^{\prime}} n_{k_{2}^{\prime}} \delta_{k_{1}^{\prime}+k_{2}^{\prime}, k_{1}+k_{2}} \delta_{k^{\prime}+k_{1}, k+k_{1}^{\prime}} \\
& +\frac{1}{\Omega^{2}} \sum_{k^{\prime}, k} e^{i\left(k^{\prime}-k\right)\left(x^{\prime}-x\right)} n_{k^{\prime}}\left(1-n_{k}\right) \\
& \times \sum_{k_{i}^{\prime}, k_{i}} h_{k_{1}^{\prime}}^{*}(t) F_{k_{1}, k_{1}^{\prime}, k^{\prime}, k}(t) h_{k_{2}}(t) F_{k_{2}^{\prime}, k_{2}, k^{\prime}, k}^{*}(t) n_{k_{1}^{\prime}} n_{k_{2}} \delta_{k_{1}^{\prime}+k_{2}^{\prime}, k_{1}+k_{2}} \delta_{k^{\prime}+k_{1}, k+k_{1}^{\prime}} \\
& +\frac{1}{\Omega^{2}} \sum_{k^{\prime}, k} e^{i\left(k^{\prime}-k\right)\left(x^{\prime}-x\right)} n_{k^{\prime}}\left(1-n_{k}\right) \\
& \times \sum_{k_{i}^{\prime}, k_{i}} h_{k_{1}}(t) F_{k_{1}^{\prime}, k_{1}, k, k^{\prime}}^{*}(t) h_{k_{2}^{\prime}}^{*}(t) F_{k_{2}, k_{2}^{\prime}, k, k^{\prime}}(t) n_{k_{1}} n_{k_{2}^{\prime}} \delta_{k_{1}^{\prime}+k_{2}^{\prime}, k_{1}+k_{2}} \delta_{k^{\prime}+k_{1}, k+k_{1}^{\prime}} \\
& +\frac{1}{\Omega^{2}} \sum_{k^{\prime}, k} e^{i\left(k^{\prime}-k\right)\left(x^{\prime}-x\right)} n_{k^{\prime}}\left(1-n_{k}\right) \\
& \times \sum_{k_{i}^{\prime}, k_{i}} h_{k_{1}}(t) F_{k_{1}^{\prime}, k_{1}, k, k^{\prime}}^{*}(t) h_{k_{2}}(t) F_{k_{2}^{\prime}, k_{2}, k^{\prime}, k}^{*}(t) n_{k_{1}} n_{k_{2}} \delta_{k_{1}^{\prime}+k_{2}^{\prime}, k_{1}+k_{2}} \delta_{k^{\prime}+k_{1}, k+k_{1}^{\prime}} \\
& +\frac{2}{\Omega^{2}} \sum_{k^{\prime}, k} e^{i\left(k^{\prime}-k\right)\left(x^{\prime}-x\right)} n_{k^{\prime}} \\
& \times \sum_{k_{i}^{\prime}, k_{i}} \mathfrak{R}\left(h_{k^{\prime}}^{*}(t) h_{k_{1}}^{*}(t) F_{k_{1}^{\prime}, k^{\prime}, k_{2}, k_{2}^{\prime}}(t) F_{k, k_{1}, k_{2}^{\prime}, k_{2}}(t)\right) \\
& \times\left(1-n_{k_{1}}\right) n_{k_{2}^{\prime}}\left(1-n_{k_{2}}\right) \delta_{k+k_{2}^{\prime}, k_{1}+k_{2}} \delta_{k^{\prime}+k_{1}, k+k_{1}^{\prime}} \\
& +\frac{1}{\Omega^{2}} \sum_{k^{\prime}, k} e^{i\left(k^{\prime}-k\right)\left(x^{\prime}-x\right)} \sum_{k_{1}, k_{2}^{\prime}, k_{2}}\left|h_{k^{\prime}}(t)\right|^{2}\left|F_{k, k_{1}, k_{2}^{\prime}, k_{2}}(t)\right|^{2} n_{k^{\prime}}\left(1-n_{k_{1}}\right) n_{k_{2}^{\prime}}\left(1-n_{k_{2}}\right) \delta_{k+k_{2}^{\prime}, k_{1}+k_{2}} \\
& +\frac{1}{\Omega^{2}} \sum_{k^{\prime}, k} e^{-i\left(k^{\prime}-k\right)\left(x^{\prime}-x\right)} \sum_{k_{1}, k_{2}^{\prime}, k_{2}}\left|h_{k^{\prime}}(t)\right|^{2}\left|F_{k, k_{1}, k_{2}^{\prime}, k_{2}}(t)\right|^{2}\left(1-n_{k^{\prime}}\right) n_{k_{1}}\left(1-n_{k_{2}^{\prime}}\right) n_{k_{2}} \delta_{k+k_{2}^{\prime}, k_{1}+k_{2}} \\
& +O\left(U^{3}\right) \text {. }
\end{aligned}
$$

At this point, one realizes how lengthy calculations can get when using unitary perturbation theory. 
Now, we insert the solutions from eqs. (5.56) - 5.58) and the result is

$$
\begin{aligned}
& C_{x^{\prime}, x}^{\uparrow \uparrow}(t)=\frac{1}{\Omega^{2}} \sum_{k^{\prime}, k} e^{i\left(k^{\prime}-k\right)\left(x^{\prime}-x\right)} n_{k^{\prime}}\left(1-n_{k}\right) \\
& -\frac{4 U^{2}}{\Omega^{4}} \sum_{k^{\prime}, k} e^{i\left(k^{\prime}-k\right)\left(x^{\prime}-x\right)} n_{k^{\prime}}\left(1-n_{k}\right) \\
& \times \sum_{k_{1}, k_{2}^{\prime}, k_{2}} \frac{1-\cos \left(\left(\Delta \epsilon_{k, k_{1}, k_{2}^{\prime}, k_{2}}\right) t\right)}{\left(\Delta \epsilon_{k, k_{1}, k_{2}^{\prime}, k_{2}}\right)^{2}} \\
& \times\left(\left(1-n_{k_{1}}\right) n_{k_{2}^{\prime}}\left(1-n_{k_{2}}\right)+n_{k_{1}}\left(1-n_{k_{2}^{\prime}}\right) n_{k_{2}}\right) \delta_{k+k_{2}^{\prime}, k_{1}+k_{2}} \\
& +\frac{2 U^{2}}{\Omega^{4}} \sum_{k^{\prime}, k} e^{i\left(k^{\prime}-k\right)\left(x^{\prime}-x\right)} n_{k^{\prime}}\left(1-n_{k}\right) \\
& \times \sum_{k_{i}^{\prime}, k_{i}} \frac{1-\cos \left(\left(\Delta \epsilon_{k^{\prime}, k_{1}^{\prime}, k_{2}^{\prime}, k_{2}}\right) t\right)-\cos \left(\left(\Delta \epsilon_{k, k_{1}, k_{2}^{\prime}, k_{2}}\right) t\right)+\cos \left(\left(\Delta \epsilon_{k^{\prime}, k, k_{1}, k_{1}^{\prime}}\right) t\right)}{\left(\Delta \epsilon_{k^{\prime}, k_{1}^{\prime}, k_{2}^{\prime}, k_{2}}\right)\left(\Delta \epsilon_{k, k_{1}, k_{2}^{\prime}, k_{2}}\right)} \\
& \times n_{k_{2}^{\prime}}\left(1-n_{k_{2}}\right) \delta_{k+k_{2}^{\prime}, k_{1}+k_{2}} \delta_{k^{\prime}+k_{1}, k+k_{1}^{\prime}} \\
& -\frac{2 U^{2}}{\Omega^{4}} \sum_{k^{\prime}, k} e^{i\left(k^{\prime}-k\right)\left(x^{\prime}-x\right)} n_{k^{\prime}}\left(1-n_{k}\right) \\
& \times \sum_{k_{i}^{\prime}, k_{i}} \frac{\Delta \epsilon_{k^{\prime}, k, k_{2}^{\prime}, k_{2}}}{\Delta \epsilon_{k_{1}^{\prime}, k_{1}, k_{2}, k_{2}^{\prime}}}\left(\frac{1-\cos \left(\left(\Delta \epsilon_{k^{\prime}, k, k_{1}^{\prime}, k_{1}}\right) t\right)}{\left(\Delta \epsilon_{k^{\prime}, k, k_{2}^{\prime}, k_{2}}\right)^{2}+\left(\Delta \epsilon_{k_{1}^{\prime}, k_{1}, k_{2}, k_{2}^{\prime}}\right)^{2}}-\frac{1-\cos \left(\left(\Delta \epsilon_{k^{\prime}, k, k_{2}^{\prime}, k_{2}}\right) t\right)}{\left(\Delta \epsilon_{k^{\prime}, k, k_{2}^{\prime}, k_{2}}\right)^{2}}\right) \\
& \times\left(n_{k_{1}^{\prime}}-n_{k_{1}}\right)\left(n_{k_{2}^{\prime}}-n_{k_{2}}\right) \delta_{k^{\prime}+k_{1}^{\prime}, k+k_{1}} \delta_{k^{\prime}+k_{2}^{\prime}, k+k_{2}} \\
& +\frac{2 U^{2}}{\Omega^{4}} \sum_{k^{\prime}, k} e^{i\left(k^{\prime}-k\right)\left(x^{\prime}-x\right)}\left(n_{k^{\prime}}\left(1-n_{k}\right)+\left(1-n_{k^{\prime}}\right) n_{k}\right) \\
& \times \sum_{k_{i}^{\prime}, k_{i}} \frac{\Delta \epsilon_{k, k_{1}, k_{2}^{\prime}, k_{2}}}{\Delta \epsilon_{k^{\prime}, k_{1}^{\prime}, k_{2}^{\prime}, k_{2}}}\left(\frac{1-\cos \left(\left(\Delta \epsilon_{k^{\prime}, k, k_{1}, k_{1}^{\prime}}\right) t\right)}{\left(\Delta \epsilon_{k^{\prime}, k_{1}^{\prime}, k_{2}^{\prime}, k_{2}}\right)^{2}+\left(\Delta \epsilon_{k, k_{1}, k_{2}^{\prime}, k_{2}}\right)^{2}}-\frac{1-\cos \left(\left(\Delta \epsilon_{k, k_{1}, k_{2}^{\prime}, k_{2}}\right) t\right)}{\left(\Delta \epsilon_{k, k_{1}, k_{2}^{\prime}, k_{2}}\right)^{2}}\right) \\
& \times n_{k_{1}}\left(n_{k_{2}^{\prime}}-n_{k_{2}}\right) \delta_{k+k_{2}^{\prime}, k_{1}+k_{2}} \delta_{k^{\prime}+k_{1}, k+k_{1}^{\prime}} \\
& +\frac{2 U^{2}}{\Omega^{4}} \sum_{k^{\prime}, k} e^{i\left(k^{\prime}-k\right)\left(x^{\prime}-x\right)} n_{k^{\prime}}\left(1-n_{k}\right) \\
& \times \sum_{k_{i}^{\prime}, k_{i}} \frac{\left(\Delta \epsilon_{k_{1}^{\prime}, k_{1}, k_{2}, k_{2}^{\prime}}\right)+\left(\Delta \epsilon_{k^{\prime}, k, k_{2}, k_{2}^{\prime}}\right)}{\left(\Delta \epsilon_{k^{\prime}, k, k_{1}, k_{1}^{\prime}}\right)}\left(\frac{1-\cos \left(\left(\Delta \epsilon_{k^{\prime}, k, k_{1}, k_{1}^{\prime}}\right) t\right)}{\left(\Delta \epsilon_{k_{1}^{\prime}, k_{1}, k_{2}, k_{2}^{\prime}}\right)^{2}+\left(\Delta \epsilon_{k^{\prime}, k, k_{2}, k_{2}^{\prime}}\right)^{2}}\right) \\
& \times\left(n_{k_{1}^{\prime}}-n_{k_{1}}\right)\left(n_{k_{2}^{\prime}}-n_{k_{2}}\right) \delta_{k^{\prime}+k_{1}, k+k_{1}^{\prime}} \delta_{k^{\prime}+k_{2}, k+k_{2}^{\prime}} \\
& +\frac{2 U^{2}}{\Omega^{4}} \sum_{k^{\prime}, k} e^{i\left(k^{\prime}-k\right)\left(x^{\prime}-x\right)}\left(n_{k^{\prime}}\left(1-n_{k}\right)+\left(1-n_{k^{\prime}}\right) n_{k}\right) \\
& \times \sum_{k_{i}^{\prime}, k_{i}} \frac{\left(\Delta \epsilon_{k^{\prime}, k_{1}^{\prime}, k_{2}^{\prime}, k_{2}}\right)+\left(\Delta \epsilon_{k, k_{1}, k_{2}^{\prime}, k_{2}}\right)}{\left(\Delta \epsilon_{k^{\prime}, k, k_{1}, k_{1}^{\prime}}\right)}\left(\frac{1-\cos \left(\left(\Delta \epsilon_{k^{\prime}, k, k_{1}, k_{1}^{\prime}}\right) t\right)}{\left(\Delta \epsilon_{k^{\prime}, k_{1}^{\prime}, k_{2}^{\prime}, k_{2}}\right)^{2}+\left(\Delta \epsilon_{k, k_{1}, k_{2}^{\prime}, k_{2}}\right)^{2}}\right) \\
& \times n_{k_{1}^{\prime}}\left(n_{k_{2}^{\prime}}-n_{k_{2}}\right) \delta_{k+k_{2}^{\prime}, k_{1}+k_{2}} \delta_{k^{\prime}+k_{1}, k+k_{1}^{\prime}}
\end{aligned}
$$




$$
\begin{aligned}
& +\frac{U^{2}}{\Omega^{4}} \sum_{k^{\prime}, k} e^{i\left(k^{\prime}-k\right)\left(x^{\prime}-x\right)} n_{k^{\prime}}\left(1-n_{k}\right) \\
& \times \sum_{k_{i}^{\prime}, k_{i}} \frac{1-\cos \left(\left(\Delta \epsilon_{k^{\prime}, k, k_{1}^{\prime}, k_{1}}\right) t\right)-\cos \left(\left(\Delta \epsilon_{k^{\prime}, k, k_{2}^{\prime}, k_{2}}\right) t\right)+\cos \left(\left(\Delta \epsilon_{k_{1}^{\prime}, k_{1}, k_{2}, k_{2}^{\prime}}\right) t\right)}{\left(\Delta \epsilon_{k^{\prime}, k, k_{1}^{\prime}, k_{1}}\right)\left(\Delta \epsilon_{k^{\prime}, k, k_{2}^{\prime}, k_{2}}\right)} \\
& \times\left(n_{k_{1}^{\prime}}-n_{k_{1}}\right)\left(n_{k_{2}^{\prime}}-n_{k_{2}}\right) \delta_{k^{\prime}+k_{1}^{\prime}, k+k_{1}} \delta_{k^{\prime}+k_{2}^{\prime}, k+k_{2}} \\
& -\frac{2 U^{2}}{\Omega^{4}} \sum_{k^{\prime}, k} e^{i\left(k^{\prime}-k\right)\left(x^{\prime}-x\right)} n_{k^{\prime}} \\
& \times \sum_{k_{i}^{\prime}, k_{i}} \frac{1-\cos \left(\left(\Delta \epsilon_{k^{\prime}, k_{1}^{\prime}, k_{2}^{\prime}, k_{2}}\right) t\right)-\cos \left(\left(\Delta \epsilon_{k, k_{1}, k_{2}^{\prime}, k_{2}}\right) t\right)+\cos \left(\left(\Delta \epsilon_{k^{\prime}, k, k_{1}, k_{1}^{\prime}}\right) t\right)}{\left(\Delta \epsilon_{k^{\prime}, k_{1}^{\prime}, k_{2}^{\prime}, k_{2}}\right)\left(\Delta \epsilon_{k, k_{1}, k_{2}^{\prime}, k_{2}}\right)} \\
& \times\left(1-n_{k_{1}}\right) n_{k_{2}^{\prime}}\left(1-n_{k_{2}}\right) \delta_{k+k_{2}^{\prime}, k_{1}+k_{2}} \delta_{k^{\prime}+k_{1}, k+k_{1}^{\prime}} \\
& +\frac{4 U^{2}}{\Omega^{4}} \sum_{k^{\prime}, k} e^{i\left(k^{\prime}-k\right)\left(x^{\prime}-x\right)} n_{k^{\prime}} \\
& \times \sum_{k_{1}, k_{2}^{\prime}, k_{2}} \frac{1-\cos \left(\left(\Delta \epsilon_{k, k_{1}, k_{2}^{\prime}, k_{2}}\right) t\right)}{\left(\Delta \epsilon_{k_{1}, k_{1}, k_{2}^{\prime}, k_{2}}\right)^{2}}\left(1-n_{k_{1}}\right) n_{k_{2}^{\prime}}\left(1-n_{k_{2}}\right) \delta_{k+k_{2}^{\prime}, k_{1}+k_{2}} \\
& +O\left(U^{3}\right) \text {. }
\end{aligned}
$$

Consistency check: variance of total spin-up particle number

For consistency, $\sum_{x^{\prime}, x} C_{x^{\prime}, x}^{\uparrow \uparrow}(t)$ should be time-independent, because by definition

$$
\sum_{x^{\prime}, x} C_{x^{\prime}, x}^{\uparrow \uparrow}(t)=\left\langle\left(N_{\uparrow}(t)\right)^{2}\right\rangle-\left(\left\langle N_{\uparrow}(t)\right\rangle\right)^{2}
$$

is the variance of the conserved total spin-up particle number. In App. B.3, we show that indeed

$$
\sum_{x^{\prime}, x} C_{x^{\prime}, x}^{\uparrow \uparrow}(t)=\sum_{k} n_{k}\left(1-n_{k}\right)+O\left(U^{3}\right)
$$

which is constant in time.

\subsection{Correlation function in equilibrium}

We have calculated the build-up of the equal-time connected density-density correlation functions after a weak interaction quench in the HUBBARD model for an initial temperature $T$, where the results are given by eq. 6.22) for antiparallel spins and by eq. (6.24) for parallel spins. An interesting question is how they relate to the correlation functions for the interacting HubBard model in equilibrium at temperature $T$, denoted by $C_{x^{\prime}, x}^{\text {eq. } \uparrow \downarrow}$ and $C_{x^{\prime}, x}^{\text {eq. } \uparrow \uparrow}$.

We can calculate equilibrium quantities in the energy-diagonal basis, at $B=\infty$, of the flow equation scheme, as indicated in sec. 2.2.1. As our reference state $\left|\psi_{0}\right\rangle$ is a thermal state of the quadratic Hamiltonian $H(B=\infty)=\tilde{H}_{0}$ specified in eq. $(5.33)$, it will be a thermal state 
of the interacting Hamiltonian if we transform it back from $B=\infty$ to $B=0$, at least in a perturbative sense. This means that for any observable $\tilde{O}-$ transformed to the basis at $B=\infty-$ its expectation value with respect to $\left|\psi_{0}\right\rangle$ is equal to the expectation value of the original observable $O(B=0)$ with respect to the thermal equilibrium state of the interacting Hamiltonian, i.e.,

$$
O^{\mathrm{eq} .}=\left\langle\psi_{0}|\tilde{O}| \psi_{0}\right\rangle
$$

To calculate the equilibrium correlation functions, we take the general expressions from eqs. (6.19) and (6.23) with the only difference that we insert the coefficients from eqs. (5.49) - (5.51).

\subsubsection{For antiparallel spins}

For antiparallel spins, this means that we modify eq. (6.19) as follows,

$$
\begin{aligned}
C_{x^{\prime}, x}^{\mathrm{eq} \cdot \uparrow \downarrow}= & \frac{1}{\Omega^{2}} \sum_{k^{\prime}, k, q^{\prime}, q} e^{i\left(k^{\prime}-k\right)\left(x^{\prime}-x\right)} \tilde{h}_{k^{\prime}}^{*} \tilde{h}_{k} \tilde{h}_{q^{\prime}}^{*} \tilde{F}_{q, q^{\prime}, k, k^{\prime}} n_{k^{\prime}}\left(1-n_{k}\right) n_{q^{\prime}} \delta_{k^{\prime}+q^{\prime}, k+q} \\
& +\frac{1}{\Omega^{2}} \sum_{k^{\prime}, k, q^{\prime}, q} e^{i\left(k^{\prime}-k\right)\left(x^{\prime}-x\right)} \tilde{h}_{k^{\prime}}^{*} \tilde{h}_{k} \tilde{h}_{q} \tilde{F}_{q^{\prime}, q, k^{\prime}, k}^{*} n_{k^{\prime}}\left(1-n_{k}\right) n_{q^{\prime}} \delta_{k^{\prime}+q^{\prime}, k+q} \\
& +\frac{1}{\Omega^{2}} \sum_{k^{\prime}, k, q^{\prime}, q} e^{i\left(k^{\prime}-k\right)\left(x^{\prime}-x\right)} \tilde{h}_{k^{\prime}} \tilde{h}_{k}^{*} \tilde{h}_{q}^{*} F_{q^{\prime}, q, k^{\prime}, k} n_{k^{\prime}}\left(1-n_{k}\right) n_{q} \delta_{k^{\prime}+q^{\prime}, k+q} \\
& +\frac{1}{\Omega^{2}} \sum_{k^{\prime}, k, q^{\prime}, q} e^{i\left(k^{\prime}-k\right)\left(x^{\prime}-x\right)} \tilde{h}_{k^{\prime}} \tilde{h}_{k}^{*} \tilde{h}_{q^{\prime}} \tilde{F}_{q, q^{\prime}, k, k^{\prime}} n_{k^{\prime}}\left(1-n_{k}\right) n_{q^{\prime}} \delta_{k^{\prime}+q^{\prime}, k+q} \\
& +O\left(U^{2}\right) .
\end{aligned}
$$

If we inserted the time-evolved expressions for $\tilde{h}_{k}(t)$ and $\tilde{F}_{k, k_{1}, k_{2}^{\prime}, k_{2}}(t)$ from eqs. (5.53) and (5.54), we would realize that the time-dependent factors cancel out meaning that the correlation function is indeed stationary. We get

$$
\begin{aligned}
C_{x^{\prime}, x}^{\mathrm{eq} \uparrow \downarrow}= & -\frac{U}{\Omega^{3}} \sum_{k^{\prime}, k, q^{\prime}, q} e^{i\left(k^{\prime}-k\right)\left(x^{\prime}-x\right)} \frac{1}{\Delta \epsilon_{q, q^{\prime}, k, k^{\prime}}} n_{k^{\prime}}\left(1-n_{k}\right) n_{q^{\prime}} \delta_{k^{\prime}+q^{\prime}, k+q} \\
& -\frac{U}{\Omega^{3}} \sum_{k^{\prime}, k, q^{\prime}, q} e^{i\left(k^{\prime}-k\right)\left(x^{\prime}-x\right)} \frac{1}{\Delta \epsilon_{q^{\prime}, q, k^{\prime}, k}} n_{k^{\prime}}\left(1-n_{k}\right) n_{q} \delta_{k^{\prime}+q^{\prime}, k+q} \\
& -\frac{U}{\Omega^{3}} \sum_{k^{\prime}, k, q^{\prime}, q} e^{i\left(k^{\prime}-k\right)\left(x^{\prime}-x\right)} \frac{1}{\Delta \epsilon_{q^{\prime}, q, k^{\prime}, k}} n_{k^{\prime}}\left(1-n_{k}\right) n_{q} \delta_{k^{\prime}+q^{\prime}, k+q} \\
& -\frac{U}{\Omega^{3}} \sum_{k^{\prime}, k, q^{\prime}, q} e^{i\left(k^{\prime}-k\right)\left(x^{\prime}-x\right)} \frac{1}{\Delta \epsilon_{q, q^{\prime}, k, k^{\prime}}} n_{k^{\prime}}\left(1-n_{k}\right) n_{q^{\prime}} \delta_{k^{\prime}+q^{\prime}, k+q} \\
& +O\left(U^{2}\right) \\
= & \frac{2 U}{\Omega^{3}} \sum_{k^{\prime}, k} e^{i\left(k^{\prime}-k\right)\left(x^{\prime}-x\right)}\left(n_{k^{\prime}}-n_{k}\right) \sum_{q^{\prime}, q} \frac{1}{\Delta \epsilon_{k^{\prime}, k, q^{\prime}, q}}\left(1-n_{q^{\prime}}\right) n_{q} \delta_{k^{\prime}+q^{\prime}, k+q} \\
& +O\left(U^{2}\right) .
\end{aligned}
$$


Now, we calculate the time average of the nonequilibrium correlation function from eq. 6.22,

$$
\overline{C_{x^{\prime}, x}^{\uparrow \downarrow}(t)} \stackrel{\text { def }}{=} \lim _{t \rightarrow \infty} \frac{1}{t} \int_{0}^{t} \mathrm{~d} t^{\prime} C_{x^{\prime}, x}^{\uparrow \downarrow}\left(t^{\prime}\right) .
$$

As our perturbative approach only covers time scales up to and including the prethermalization regime, $t \lesssim \rho_{\mathrm{F}}^{-1} U^{-2}$, this time average equals the prethermalization value $C_{x^{\prime}, x}^{\text {pre. } \downarrow}$ of the correlation function. The integration to $t=\infty$ makes the initial transient of the correlation function play no role for the time average.

It turns out that the cos-function in eq. (6.22) drops out, so that

$$
\begin{aligned}
\overline{C_{x^{\prime}, x}^{\uparrow \downarrow}(t)}= & \frac{2 U}{\Omega^{3}} \sum_{k^{\prime}, k} e^{i\left(k^{\prime}-k\right)\left(x^{\prime}-x\right)}\left(n_{k^{\prime}}-n_{k}\right) \sum_{q^{\prime}, q} \frac{1}{\Delta \epsilon_{k^{\prime}, k, q^{\prime}, q}}\left(1-n_{q^{\prime}}\right) n_{q} \delta_{k^{\prime}+q^{\prime}, k+q} \\
& +O\left(U^{2}\right) .
\end{aligned}
$$

This is exactly the same expression as for the equilibrium correlation function. Hence, we have found that, to leading order, the prethermalization value of the nonequilibrium function with an initial temperature $T$ is equal to the equilibrium value at the same temperature,

$$
\begin{aligned}
C_{x^{\prime}, x}^{\text {pre. } \uparrow \downarrow} & \equiv \overline{C_{x^{\prime}, x}^{\uparrow \downarrow}(t)} \\
& =C_{x^{\prime}, x}^{\text {eq. } \uparrow \downarrow}+O\left(U^{2}\right) .
\end{aligned}
$$

We note that this cannot be the thermal value of the nonequilibrium correlation function because the heating effect of the quench will increase the temperature in the long-time limit. 


\subsubsection{For parallel spins}

For parallel spins, the strategy is the same as above. We modify eq. 6.23 by putting a tilde on the coefficients. Again, the time-dependencies would cancel out, and we get

$$
\begin{aligned}
& C_{x^{\prime}, x}^{\mathrm{eq} \cdot \uparrow \uparrow}=\frac{1}{\Omega^{2}} \sum_{k^{\prime}, k} e^{i\left(k^{\prime}-k\right)\left(x^{\prime}-x\right)} n_{k^{\prime}}\left(1-n_{k}\right) \\
& -\frac{2 U^{2}}{\Omega^{4}} \sum_{k^{\prime}, k} e^{i\left(k^{\prime}-k\right)\left(x^{\prime}-x\right)} n_{k^{\prime}}\left(1-n_{k}\right) \\
& \times \sum_{k_{1}, k_{2}^{\prime}, k_{2}} \frac{1}{\left(\Delta \epsilon_{k, k_{1}, k_{2}^{\prime}, k_{2}}\right)^{2}}\left(\left(1-n_{k_{1}}\right) n_{k_{2}^{\prime}}\left(1-n_{k_{2}}\right)+n_{k_{1}}\left(1-n_{k_{2}^{\prime}}\right) n_{k_{2}}\right) \delta_{k+k_{2}^{\prime}, k_{1}+k_{2}} \\
& +\frac{2 U^{2}}{\Omega^{4}} \sum_{k^{\prime}, k} e^{i\left(k^{\prime}-k\right)\left(x^{\prime}-x\right)} n_{k^{\prime}}\left(1-n_{k}\right) \\
& \times \sum_{k_{i}^{\prime}, k_{i}} \frac{1}{\left(\Delta \epsilon_{k^{\prime}, k_{1}^{\prime}, k_{2}^{\prime}, k_{2}}\right)\left(\Delta \epsilon_{k, k_{1}, k_{2}^{\prime}, k_{2}}\right)} n_{k_{2}^{\prime}}\left(1-n_{k_{2}}\right) \delta_{k+k_{2}^{\prime}, k_{1}+k_{2}} \delta_{k^{\prime}+k_{1}, k+k_{1}^{\prime}} \\
& -\frac{2 U^{2}}{\Omega^{4}} \sum_{k^{\prime}, k} e^{i\left(k^{\prime}-k\right)\left(x^{\prime}-x\right)} n_{k^{\prime}}\left(1-n_{k}\right) \\
& \times \sum_{k_{i}^{\prime}, k_{i}} \frac{\Delta \epsilon_{k^{\prime}, k, k_{2}^{\prime}, k_{2}}}{\Delta \epsilon_{k_{1}^{\prime}, k_{1}, k_{2}, k_{2}^{\prime}}}\left(\frac{1}{\left(\Delta \epsilon_{k^{\prime}, k, k_{2}^{\prime}, k_{2}}\right)^{2}+\left(\Delta \epsilon_{k_{1}^{\prime}, k_{1}, k_{2}, k_{2}^{\prime}}\right)^{2}}-\frac{1}{\left(\Delta \epsilon_{k^{\prime}, k, k_{2}^{\prime}, k_{2}}\right)^{2}}\right) \\
& \times\left(n_{k_{1}^{\prime}}-n_{k_{1}}\right)\left(n_{k_{2}^{\prime}}-n_{k_{2}}\right) \delta_{k^{\prime}+k_{1}^{\prime}, k+k_{1}} \delta_{k^{\prime}+k_{2}^{\prime}, k+k_{2}} \\
& +\frac{2 U^{2}}{\Omega^{4}} \sum_{k^{\prime}, k} e^{i\left(k^{\prime}-k\right)\left(x^{\prime}-x\right)}\left(n_{k^{\prime}}\left(1-n_{k}\right)+\left(1-n_{k^{\prime}}\right) n_{k}\right) \\
& \times \sum_{k_{i}^{\prime}, k_{i}} \frac{\Delta \epsilon_{k, k_{1}, k_{2}^{\prime}, k_{2}}}{\Delta \epsilon_{k^{\prime}, k_{1}^{\prime}, k_{2}^{\prime}, k_{2}}}\left(\frac{1}{\left(\Delta \epsilon_{k^{\prime}, k_{1}^{\prime}, k_{2}^{\prime}, k_{2}}\right)^{2}+\left(\Delta \epsilon_{k, k_{1}, k_{2}^{\prime}, k_{2}}\right)^{2}}-\frac{1}{\left(\Delta \epsilon_{k, k_{1}, k_{2}^{\prime}, k_{2}}\right)^{2}}\right) \\
& \times n_{k_{1}}\left(n_{k_{2}^{\prime}}-n_{k_{2}}\right) \delta_{k+k_{2}^{\prime}, k_{1}+k_{2}} \delta_{k^{\prime}+k_{1}, k+k_{1}^{\prime}} \\
& +\frac{2 U^{2}}{\Omega^{4}} \sum_{k^{\prime}, k} e^{i\left(k^{\prime}-k\right)\left(x^{\prime}-x\right)} n_{k^{\prime}}\left(1-n_{k}\right) \\
& \times \sum_{k_{i}^{\prime}, k_{i}} \frac{\left(\Delta \epsilon_{k_{1}^{\prime}, k_{1}, k_{2}, k_{2}^{\prime}}\right)+\left(\Delta \epsilon_{k^{\prime}, k, k_{2}, k_{2}^{\prime}}\right)}{\left(\Delta \epsilon_{k^{\prime}, k, k_{1}, k_{1}^{\prime}}\right)}\left(\frac{1}{\left(\Delta \epsilon_{k_{1}^{\prime}, k_{1}, k_{2}, k_{2}^{\prime}}\right)^{2}+\left(\Delta \epsilon_{k^{\prime}, k, k_{2}, k_{2}^{\prime}}\right)^{2}}\right) \\
& \times\left(n_{k_{1}^{\prime}}-n_{k_{1}}\right)\left(n_{k_{2}^{\prime}}-n_{k_{2}}\right) \delta_{k^{\prime}+k_{1}, k+k_{1}^{\prime}} \delta_{k^{\prime}+k_{2}, k+k_{2}^{\prime}} \\
& +\frac{2 U^{2}}{\Omega^{4}} \sum_{k^{\prime}, k} e^{i\left(k^{\prime}-k\right)\left(x^{\prime}-x\right)}\left(n_{k^{\prime}}\left(1-n_{k}\right)+\left(1-n_{k^{\prime}}\right) n_{k}\right) \\
& \times \sum_{k_{i}^{\prime}, k_{i}} \frac{\left(\Delta \epsilon_{k^{\prime}, k_{1}^{\prime}, k_{2}^{\prime}, k_{2}}\right)+\left(\Delta \epsilon_{k, k_{1}, k_{2}^{\prime}, k_{2}}\right)}{\left(\Delta \epsilon_{k^{\prime}, k, k_{1}, k_{1}^{\prime}}\right)}\left(\frac{1}{\left(\Delta \epsilon_{k^{\prime}, k_{1}^{\prime}, k_{2}^{\prime}, k_{2}}\right)^{2}+\left(\Delta \epsilon_{k, k_{1}, k_{2}^{\prime}, k_{2}}\right)^{2}}\right) \\
& \times n_{k_{1}^{\prime}}\left(n_{k_{2}^{\prime}}-n_{k_{2}}\right) \delta_{k+k_{2}^{\prime}, k_{1}+k_{2}} \delta_{k^{\prime}+k_{1}, k+k_{1}^{\prime}}
\end{aligned}
$$




$$
\begin{aligned}
& +\frac{U^{2}}{\Omega^{4}} \sum_{k^{\prime}, k} e^{i\left(k^{\prime}-k\right)\left(x^{\prime}-x\right)} n_{k^{\prime}}\left(1-n_{k}\right) \\
& \quad \times \sum_{k_{i}^{\prime}, k_{i}} \frac{1}{\left(\Delta \epsilon_{k^{\prime}, k^{\prime}, k_{1}^{\prime}, k_{1}}\right)\left(\Delta \epsilon_{k^{\prime}, k^{\prime}, k_{2}^{\prime}, k_{2}}\right)}\left(n_{k_{1}^{\prime}}-n_{k_{1}}\right)\left(n_{k_{2}^{\prime}}-n_{k_{2}}\right) \delta_{k^{\prime}+k_{1}^{\prime}, k+k_{1}} \delta_{k^{\prime}+k_{2}^{\prime}, k+k_{2}} \\
& -\frac{2 U^{2}}{\Omega^{4}} \sum_{k^{\prime}, k} e^{i\left(k^{\prime}-k\right)\left(x^{\prime}-x\right)} n_{k^{\prime}} \\
& \quad \times \sum_{k_{i}^{\prime}, k_{i}} \frac{1}{\left(\Delta \epsilon_{k^{\prime}, k_{1}^{\prime}, k_{2}^{\prime}, k_{2}}\right)\left(\Delta \epsilon_{k^{\prime}, k_{1}, k_{2}^{\prime}, k_{2}}\right)}\left(1-n_{k_{1}}\right) n_{k_{2}^{\prime}}\left(1-n_{k_{2}}\right) \delta_{k_{k}+k_{2}^{\prime}, k_{1}+k_{2}} \delta_{k^{\prime}+k_{1}, k+k_{1}^{\prime}} \\
& +\sum_{k^{\prime}, k} e^{i\left(k^{\prime}-k\right)\left(x^{\prime}-x\right)} n_{k^{\prime}} \\
& \quad \times \sum_{k_{1}, k_{2}^{\prime}, k_{2}} \frac{1}{\left(\Delta \epsilon_{k, k_{1}, k_{2}^{\prime}, k_{2}}\right)^{2}}\left(1-n_{k_{1}}\right) n_{k_{2}^{\prime}}\left(1-n_{k_{2}}\right) \delta_{k+k_{2}^{\prime}, k_{1}+k_{2}} \\
& +\boldsymbol{O}\left(U^{3}\right) .
\end{aligned}
$$

When taking the time average of the nonequilibrium function from eq. (6.24), the cos-functions drop out again, but we cannot directly relate the nonequilibrium case to the equation above because different terms have different prefactors. Indeed, we are left with a difference

$$
\begin{aligned}
\overline{C_{x^{\prime}, x}^{\uparrow \uparrow}(t)}-C_{x^{\prime}, x}^{\mathrm{eq} \uparrow \uparrow}= & -\frac{2 U^{2}}{\Omega^{4}} \sum_{k^{\prime}, k} e^{i\left(k^{\prime}-k\right)\left(x^{\prime}-x\right)} n_{k^{\prime}}\left(1-n_{k}\right) \\
& \times \sum_{k_{1}, k_{2}^{\prime}, k_{2}} \frac{1}{\left(\Delta \epsilon_{k_{k}, k_{1}, k_{2}^{\prime}, k_{2}}\right)^{2}} \\
& \times\left(\left(1-n_{k_{1}}\right) n_{k_{2}^{\prime}}\left(1-n_{k_{2}}\right)+n_{k_{1}}\left(1-n_{k_{2}^{\prime}}\right) n_{k_{2}}\right) \delta_{k+k_{2}^{\prime}, k_{1}+k_{2}} \\
& +\frac{2 U^{2}}{\Omega^{4}} \sum_{k^{\prime}, k} e^{i\left(k^{\prime}-k\right)\left(x^{\prime}-x\right)} n_{k^{\prime}} \\
& \times \sum_{k_{1}, k_{2}^{\prime}, k_{2}} \frac{1}{\left(\Delta \epsilon_{k^{\prime}, k_{1}, k_{2}^{\prime}, k_{2}}\right)^{2}}\left(1-n_{k_{1}}\right) n_{k_{2}^{\prime}}\left(1-n_{k_{2}}\right) \delta_{k+k_{2}^{\prime}, k_{1}+k_{2}} \\
& +O\left(U^{3}\right) .
\end{aligned}
$$

However, in the next section we will evaluate the long-range part of the parallel-spin correlation functions, i.e., the behavior for $\left|x^{\prime}-x\right| \rightarrow \infty$, where we will use the limit of infinite spatial dimensions. With this further approximation, we will be able to compare the equilibrium result to the prethermalization value of the nonequilibrium correlation function.

\subsection{Correlation function in infinite dimensions}

We have seen that the correlation functions for parallel spins from eqs. $(6.24)$ and $(6.33)$ are very extensive in their analytical structure as written down so far. We will see that their spatial decay for $\left|x^{\prime}-x\right| \rightarrow \infty$ differs from the antiparallel-spin correlation functions and the 
susceptibilities. In order to get more analytical insight into the parallel-spin correlation function, we take the limit of infinite spatial dimensions that we have introduced in sec. 3.2 and that simplifies the manifold $k$-summations.

In this limit, we will analyze the behavior of the FOURIER transformation to momentum space for small momentum because this is closely related to the decay behavior for large distances $\left|x^{\prime}-x\right|$ in real space (cf. sec. 7.2). In the small-momentum limit we will be able to directly compare the equilibrium correlation function to the prethermalization value of the nonequilibrium function.

\subsubsection{FOURIER transformation to momentum space}

We will first perform a FOURIER transformation of the correlation functions from eqs. (6.24) and 6.33 to momentum space, where the dependence on the spatial distance $x^{\prime}-x$ will be replaced by a dependence on the momentum $q$. The FoURIER transformation is defined as

$$
\hat{C}_{q}^{\uparrow \uparrow}(t) \stackrel{\text { def }}{=} \frac{1}{\Omega} \sum_{x^{\prime}-x} e^{-i q\left(x^{\prime}-x\right)} C_{x^{\prime}, x}^{\uparrow \uparrow}(t) .
$$

Looking at eqs. (6.24) and 6.33), this effectively means that we get a $\delta_{q, k^{\prime}-k}$ from the summation over $x^{\prime}-x$ so that $k^{\prime}$ can be replaced by $k+q$.

\section{Nonequilibrium correlation function}

For the nonequilibrium correlation function from eq. (6.24) we get

$$
\begin{aligned}
& \hat{C}_{q}^{\uparrow}(t)=\frac{1}{\Omega^{2}} \sum_{k} n_{k+q}\left(1-n_{k}\right) \\
& -\frac{4 U^{2}}{\Omega^{4}} \sum_{k} n_{k+q}\left(1-n_{k}\right) \\
& \times \sum_{k_{1}, k_{2}^{\prime}, k_{2}} \frac{1-\cos \left(\left(\Delta \epsilon_{k, k_{1}, k_{2}^{\prime}, k_{2}}\right) t\right)}{\left(\Delta \epsilon_{k, k_{1}, k_{2}^{\prime}, k_{2}}\right)^{2}} \\
& \times\left(\left(1-n_{k_{1}}\right) n_{k_{2}^{\prime}}\left(1-n_{k_{2}}\right)+n_{k_{1}}\left(1-n_{k_{2}^{\prime}}\right) n_{k_{2}}\right) \delta_{k+k_{2}^{\prime}, k_{1}+k_{2}} \\
& +\frac{2 U^{2}}{\Omega^{4}} \sum_{k} n_{k+q}\left(1-n_{k}\right) \\
& \times \sum_{k_{i}^{\prime}, k_{i}} \frac{1-\cos \left(\left(\Delta \epsilon_{k+q, k_{1}^{\prime}, k_{2}^{\prime}, k_{2}}\right) t\right)-\cos \left(\left(\Delta \epsilon_{k, k_{1}, k_{2}^{\prime}, k_{2}}\right) t\right)+\cos \left(\left(\Delta \epsilon_{k+q, k, k_{1}, k_{1}^{\prime}}\right) t\right)}{\left(\Delta \epsilon_{k+q, k_{1}^{\prime}, k_{2}^{\prime}, k_{2}}\right)\left(\Delta \epsilon_{k, k_{1}, k_{2}^{\prime}, k_{2}}\right)} \\
& \times n_{k_{2}^{\prime}}\left(1-n_{k_{2}}\right) \delta_{k+k_{2}^{\prime}, k_{1}+k_{2}} \delta_{k_{1}^{\prime}-k_{1}, q} \\
& -\frac{2 U^{2}}{\Omega^{4}} \sum_{k} n_{k+q}\left(1-n_{k}\right) \\
& \times \sum_{k_{i}^{\prime}, k_{i}} \frac{\Delta \epsilon_{k+q, k, k_{2}^{\prime}, k_{2}}}{\Delta \epsilon_{k_{1}^{\prime}, k_{1}, k_{2}, k_{2}^{\prime}}}\left(\frac{1-\cos \left(\left(\Delta \epsilon_{k+q, k^{\prime}, k_{1}^{\prime}, k_{1}}\right) t\right)}{\left(\Delta \epsilon_{k+q, k^{\prime}, k_{2}^{\prime}, k_{2}}\right)^{2}+\left(\Delta \epsilon_{k_{1}^{\prime}, k_{1}, k_{2}, k_{2}^{\prime}}\right)^{2}}-\frac{1-\cos \left(\left(\Delta \epsilon_{k+q, k, k_{2}^{\prime}, k_{2}}\right) t\right)}{\left(\Delta \epsilon_{k+q, k, k_{2}^{\prime}, k_{2}}\right)^{2}}\right) \\
& \times\left(n_{k_{1}^{\prime}}-n_{k_{1}}\right)\left(n_{k_{2}^{\prime}}-n_{k_{2}}\right) \delta_{k_{1}^{\prime}-k_{1}, q} \delta_{k_{2}^{\prime}-k_{2}, q}
\end{aligned}
$$




$$
\begin{aligned}
& +\frac{2 U^{2}}{\Omega^{4}} \sum_{k}\left(n_{k+q}\left(1-n_{k}\right)+\left(1-n_{k+q}\right) n_{k}\right) \\
& \times \sum_{k_{i}^{\prime}, k_{i}} \frac{\Delta \epsilon_{k, k_{1}, k_{2}^{\prime}, k_{2}}}{\Delta \epsilon_{k+q, k_{1}^{\prime}, k_{2}^{\prime}, k_{2}}}\left(\frac{1-\cos \left(\left(\Delta \epsilon_{k+q, k, k_{1}, k_{1}^{\prime}}\right) t\right)}{\left(\Delta \epsilon_{k+q, k_{1}^{\prime}, k_{2}^{\prime}, k_{2}}\right)^{2}+\left(\Delta \epsilon_{k, k_{1}, k_{2}^{\prime}, k_{2}}\right)^{2}}-\frac{1-\cos \left(\left(\Delta \epsilon_{k, k_{1}, k_{2}^{\prime}, k_{2}}\right) t\right)}{\left(\Delta \epsilon_{k, k_{1}, k_{2}^{\prime}, k_{2}}\right)^{2}}\right) \\
& \times n_{k_{1}}\left(n_{k_{2}^{\prime}}-n_{k_{2}}\right) \delta_{k+k_{2}^{\prime}, k_{1}+k_{2}} \delta_{k_{1}^{\prime}-k_{1}, q} \\
& +\frac{2 U^{2}}{\Omega^{4}} \sum_{k} n_{k+q}\left(1-n_{k}\right) \\
& \times \sum_{k_{i}^{\prime}, k_{i}} \frac{\left(\Delta \epsilon_{k_{1}^{\prime}, k_{1}, k_{2}, k_{2}^{\prime}}\right)+\left(\Delta \epsilon_{k+q, k, k_{2}, k_{2}^{\prime}}\right)}{\left(\Delta \epsilon_{k+q, k, k_{1}, k_{1}^{\prime}}\right)}\left(\frac{1-\cos \left(\left(\Delta \epsilon_{k+q, k, k_{1}, k_{1}^{\prime}}\right) t\right)}{\left(\Delta \epsilon_{k_{1}^{\prime}, k_{1}, k_{2}, k_{2}^{\prime}}\right)^{2}+\left(\Delta \epsilon_{k+q, k, k_{2}, k_{2}^{\prime}}\right)^{2}}\right) \\
& \times\left(n_{k_{1}^{\prime}}-n_{k_{1}}\right)\left(n_{k_{2}^{\prime}}-n_{k_{2}}\right) \delta_{k_{1}^{\prime}-k_{1}, q} \delta_{k_{2}^{\prime}-k_{2}, q} \\
& +\frac{2 U^{2}}{\Omega^{4}} \sum_{k}\left(n_{k+q}\left(1-n_{k}\right)+\left(1-n_{k+q}\right) n_{k}\right) \\
& \times \sum_{k_{i}^{\prime}, k_{i}} \frac{\left(\Delta \epsilon_{k+q, k_{1}^{\prime}, k_{2}^{\prime}, k_{2}}\right)+\left(\Delta \epsilon_{k, k_{1}, k_{2}^{\prime}, k_{2}}\right)}{\left(\Delta \epsilon_{k+q, k, k_{1}, k_{1}^{\prime}}\right)}\left(\frac{1-\cos \left(\left(\Delta \epsilon_{k+q, k, k_{1}, k_{1}^{\prime}}\right) t\right)}{\left(\Delta \epsilon_{k+q, k_{1}^{\prime}, k_{2}^{\prime}, k_{2}}\right)^{2}+\left(\Delta \epsilon_{k, k_{1}, k_{2}^{\prime}, k_{2}}\right)^{2}}\right) \\
& \times n_{k_{1}^{\prime}}\left(n_{k_{2}^{\prime}}-n_{k_{2}}\right) \delta_{k+k_{2}^{\prime}, k_{1}+k_{2}} \delta_{k_{1}^{\prime}-k_{1}, q} \\
& +\frac{U^{2}}{\Omega^{4}} \sum_{k} n_{k+q}\left(1-n_{k}\right) \\
& \times \sum_{k_{i}^{\prime}, k_{i}} \frac{1-\cos \left(\left(\Delta \epsilon_{k+q, k, k_{1}^{\prime}, k_{1}}\right) t\right)-\cos \left(\left(\Delta \epsilon_{k+q, k, k_{2}^{\prime}, k_{2}}\right) t\right)+\cos \left(\left(\Delta \epsilon_{k_{1}^{\prime}, k_{1}, k_{2}, k_{2}^{\prime}}\right) t\right)}{\left(\Delta \epsilon_{k+q, k, k_{1}^{\prime}, k_{1}}\right)\left(\Delta \epsilon_{k+q, k, k_{2}^{\prime}, k_{2}}\right)} \\
& \times\left(n_{k_{1}^{\prime}}-n_{k_{1}}\right)\left(n_{k_{2}^{\prime}}-n_{k_{2}}\right) \delta_{k_{1}^{\prime}-k_{1}, q} \delta_{k_{2}^{\prime}-k_{2}, q} \\
& -\frac{2 U^{2}}{\Omega^{4}} \sum_{k} n_{k+q} \\
& \times \sum_{k_{i}^{\prime}, k_{i}} \frac{1-\cos \left(\left(\Delta \epsilon_{k+q, k_{1}^{\prime}, k_{2}^{\prime}, k_{2}}\right) t\right)-\cos \left(\left(\Delta \epsilon_{k, k_{1}, k_{2}^{\prime}, k_{2}}\right) t\right)+\cos \left(\left(\Delta \epsilon_{k+q, k, k_{1}, k_{1}^{\prime}}\right) t\right)}{\left(\Delta \epsilon_{k+q, k_{1}^{\prime}, k_{2}^{\prime}, k_{2}}\right)\left(\Delta \epsilon_{k, k_{1}, k_{2}^{\prime}, k_{2}}\right)} \\
& \times\left(1-n_{k_{1}}\right) n_{k_{2}^{\prime}}\left(1-n_{k_{2}}\right) \delta_{k+k_{2}^{\prime}, k_{1}+k_{2}} \delta_{k_{1}^{\prime}-k_{1}, q} \\
& +\frac{4 U^{2}}{\Omega^{4}} \sum_{k} n_{k+q} \\
& \times \sum_{k_{1}, k_{2}^{\prime}, k_{2}} \frac{1-\cos \left(\left(\Delta \epsilon_{k, k_{1}, k_{2}^{\prime}, k_{2}}\right) t\right)}{\left(\Delta \epsilon_{k_{1}, k_{1}, k_{2}^{\prime}, k_{2}}\right)^{2}}\left(1-n_{k_{1}}\right) n_{k_{2}^{\prime}}\left(1-n_{k_{2}}\right) \delta_{k+k_{2}^{\prime}, k_{1}+k_{2}} \\
& +O\left(U^{3}\right) \text {. }
\end{aligned}
$$




\section{Equilibrium correlation function}

Analogously, we get for the equilibrium correlation function from eq. 6.33,

$$
\begin{aligned}
& \hat{C}_{q}^{\text {eq } \cdot \uparrow}=\frac{1}{\Omega^{2}} \sum_{k} n_{k+q}\left(1-n_{k}\right) \\
& -\frac{2 U^{2}}{\Omega^{4}} \sum_{k} n_{k+q}\left(1-n_{k}\right) \\
& \times \sum_{k_{1}, k_{2}^{\prime}, k_{2}} \frac{1}{\left(\Delta \epsilon_{k, k_{1}, k_{2}^{\prime}, k_{2}}\right)^{2}}\left(\left(1-n_{k_{1}}\right) n_{k_{2}^{\prime}}\left(1-n_{k_{2}}\right)+n_{k_{1}}\left(1-n_{k_{2}^{\prime}}\right) n_{k_{2}}\right) \delta_{k+k_{2}^{\prime}, k_{1}+k_{2}} \\
& +\frac{2 U^{2}}{\Omega^{4}} \sum_{k} n_{k+q}\left(1-n_{k}\right) \\
& \times \sum_{k_{i}^{\prime}, k_{i}} \frac{1}{\left(\Delta \epsilon_{k+q, k_{1}^{\prime}, k_{2}^{\prime}, k_{2}}\right)\left(\Delta \epsilon_{k, k_{1}, k_{2}^{\prime}, k_{2}}\right)} n_{k_{2}^{\prime}}\left(1-n_{k_{2}}\right) \delta_{k+k_{2}^{\prime}, k_{1}+k_{2}} \delta_{k_{1}^{\prime}-k_{1}, q} \\
& -\frac{2 U^{2}}{\Omega^{4}} \sum_{k} n_{k+q}\left(1-n_{k}\right) \\
& \times \sum_{k_{i}^{\prime}, k_{i}} \frac{\Delta \epsilon_{k+q, k, k_{2}^{\prime}, k_{2}}}{\Delta \epsilon_{k_{1}^{\prime}, k_{1}, k_{2}, k_{2}^{\prime}}}\left(\frac{1}{\left(\Delta \epsilon_{k+q, k, k_{2}^{\prime}, k_{2}}\right)^{2}+\left(\Delta \epsilon_{k_{1}^{\prime}, k_{1}, k_{2}, k_{2}^{\prime}}\right)^{2}}-\frac{1}{\left(\Delta \epsilon_{k+q, k, k_{2}, k_{2}}\right)^{2}}\right) \\
& \times\left(n_{k_{1}^{\prime}}-n_{k_{1}}\right)\left(n_{k_{2}^{\prime}}-n_{k_{2}}\right) \delta_{k_{1}^{\prime}-k_{1}, q} \delta_{k_{2}^{\prime}-k_{2}, q} \\
& +\frac{2 U^{2}}{\Omega^{4}} \sum_{k}\left(n_{k+q}\left(1-n_{k}\right)+\left(1-n_{k+q}\right) n_{k}\right) \\
& \times \sum_{k_{i}^{\prime}, k_{i}} \frac{\Delta \epsilon_{k, k_{1}, k_{2}^{\prime}, k_{2}}}{\Delta \epsilon_{k+q, k_{1}^{\prime}, k_{2}^{\prime}, k_{2}}}\left(\frac{1}{\left(\Delta \epsilon_{k+q, k_{1}^{\prime}, k_{2}^{\prime}, k_{2}}\right)^{2}+\left(\Delta \epsilon_{k, k_{1}, k_{2}^{\prime}, k_{2}}\right)^{2}}-\frac{1}{\left(\Delta \epsilon_{k, k_{1}, k_{2}^{\prime}, k_{2}}\right)^{2}}\right) \\
& \times n_{k_{1}}\left(n_{k_{2}^{\prime}}-n_{k_{2}}\right) \delta_{k+k_{2}^{\prime}, k_{1}+k_{2}} \delta_{k_{1}^{\prime}-k_{1}, q} \\
& +\frac{2 U^{2}}{\Omega^{4}} \sum_{k} n_{k+q}\left(1-n_{k}\right) \\
& \times \sum_{k_{i}^{\prime}, k_{i}} \frac{\left(\Delta \epsilon_{k_{1}^{\prime}, k_{1}, k_{2}, k_{2}^{\prime}}\right)+\left(\Delta \epsilon_{k+q, k, k_{2}, k_{2}^{\prime}}\right)}{\left(\Delta \epsilon_{k+q, k, k_{1}, k_{1}^{\prime}}\right)}\left(\frac{1}{\left(\Delta \epsilon_{k_{1}^{\prime}, k_{1}, k_{2}, k_{2}^{\prime}}\right)^{2}+\left(\Delta \epsilon_{k+q, k, k_{2}, k_{2}^{\prime}}\right)^{2}}\right) \\
& \times\left(n_{k_{1}^{\prime}}-n_{k_{1}}\right)\left(n_{k_{2}^{\prime}}-n_{k_{2}}\right) \delta_{k_{1}^{\prime}-k_{1}, q} \delta_{k_{2}^{\prime}-k_{2}, q} \\
& +\frac{2 U^{2}}{\Omega^{4}} \sum_{k}\left(n_{k+q}\left(1-n_{k}\right)+\left(1-n_{k+q}\right) n_{k}\right) \\
& \times \sum_{k_{i}^{\prime}, k_{i}} \frac{\left(\Delta \epsilon_{k+q, k_{1}^{\prime}, k_{2}^{\prime}, k_{2}}\right)+\left(\Delta \epsilon_{k, k_{1}, k_{2}^{\prime}, k_{2}}\right)}{\left(\Delta \epsilon_{k+q, k, k_{1}, k_{1}^{\prime}}\right)}\left(\frac{1}{\left(\Delta \epsilon_{k+q, k_{1}^{\prime}, k_{2}^{\prime}, k_{2}}\right)^{2}+\left(\Delta \epsilon_{k, k_{1}, k_{2}^{\prime}, k_{2}}\right)^{2}}\right) \\
& \times n_{k_{1}^{\prime}}\left(n_{k_{2}^{\prime}}-n_{k_{2}}\right) \delta_{k+k_{2}^{\prime}, k_{1}+k_{2}} \delta_{k_{1}^{\prime}-k_{1}, q}
\end{aligned}
$$




$$
\begin{aligned}
& +\frac{U^{2}}{\Omega^{4}} \sum_{k} n_{k+q}\left(1-n_{k}\right) \sum_{k_{i}^{\prime}, k_{i}} \frac{1}{\left(\Delta \epsilon_{k+q, k, k_{1}^{\prime}, k_{1}}\right)\left(\Delta \epsilon_{k+q, k, k_{2}^{\prime}, k_{2}}\right)}\left(n_{k_{1}^{\prime}}-n_{k_{1}}\right)\left(n_{k_{2}^{\prime}}-n_{k_{2}}\right) \delta_{k_{1}^{\prime}-k_{1}, q} \delta_{k_{2}^{\prime}-k_{2}, q} \\
& -\frac{2 U^{2}}{\Omega^{4}} \sum_{k} n_{k+q} \sum_{k_{i}^{\prime}, k_{i}} \frac{1}{\left(\Delta \epsilon_{k+q, k_{1}^{\prime}, k_{2}^{\prime}, k_{2}}\right)\left(\Delta \epsilon_{k^{\prime}, k_{1}, k_{2}^{\prime}, k_{2}}\right)}\left(1-n_{k_{1}}\right) n_{k_{2}^{\prime}}\left(1-n_{k_{2}}\right) \delta_{k+k_{2}^{\prime}, k_{1}+k_{2}} \delta_{k_{1}^{\prime}-k_{1}, q} \\
& +\frac{2 U^{2}}{\Omega^{4}} \sum_{k} n_{k+q} \sum_{k_{1}, k_{2}^{\prime}, k_{2}} \frac{1}{\left(\Delta \epsilon_{k^{\prime}, k_{1}, k_{2}^{\prime}, k_{2}}\right)^{2}}\left(1-n_{k_{1}}\right) n_{k_{2}^{\prime}}\left(1-n_{k_{2}}\right) \delta_{k_{+}+k_{2}^{\prime}, k_{1}+k_{2}} \\
& +\boldsymbol{O}\left(U^{3}\right) .
\end{aligned}
$$

\subsubsection{Energy integrals in infinite spatial dimensions}

Now, we take the limit of infinite spatial dimensions, based on the considerations made in sec. 3.2. Hence, we can introduce energy integrals,

$$
\sum_{k_{i}} \ldots \rightarrow \int \mathrm{d} \epsilon_{i} \sum_{k_{i}} \delta\left(\epsilon_{i}-\epsilon_{k_{i}}\right) \ldots,
$$

and make use of eq. (3.16), i.e.,

$$
\begin{aligned}
\sum_{k_{1}, k_{2}, k_{3}} \delta\left(\epsilon_{1}-\epsilon_{k_{1}}\right) \delta\left(\epsilon_{2}-\epsilon_{k_{2}}\right) \delta\left(\epsilon_{3}-\epsilon_{k_{3}}\right) \delta_{k+k_{3}, k_{1}+k_{2}} & \stackrel{d \rightarrow \infty}{=} \frac{1}{\Omega} \sum_{k_{1}, k_{2}, k_{3}} \delta\left(\epsilon_{1}-\epsilon_{k_{1}}\right) \delta\left(\epsilon_{2}-\epsilon_{k_{2}}\right) \delta\left(\epsilon_{3}-\epsilon_{k_{3}}\right) \\
& =\Omega^{2} D\left(\epsilon_{1}\right) D\left(\epsilon_{2}\right) D\left(\epsilon_{3}\right)
\end{aligned}
$$

Furthermore, we will use the relation

$$
\begin{aligned}
\sum_{k_{1}, k_{2}} \delta\left(\epsilon_{1}-\epsilon_{k_{1}}\right) \delta\left(\epsilon_{2}-\epsilon_{k_{2}}\right) \delta_{k_{1}-k_{2}, k} \stackrel{d \rightarrow \infty}{=} \frac{1}{\Omega} \sum_{k_{1}, k_{2}} \delta\left(\epsilon_{1}-\epsilon_{k_{1}}\right) \delta\left(\epsilon_{2}-\epsilon_{k_{2}}\right) \\
=\Omega D\left(\epsilon_{1}\right) D\left(\epsilon_{2}\right),
\end{aligned}
$$

which only holds if $k \neq \overrightarrow{0}[89,112]$. This means that from now on we restrict the domain of the FOURIER transformed correlation functions to values $q \neq \overrightarrow{0}$. 


\section{Nonequilibrium correlation function}

For the nonequilibrium correlation function, we get

$$
\begin{aligned}
& \hat{C}_{q}^{\uparrow \uparrow}(t) \stackrel{d=\infty}{=} \frac{1}{\Omega^{2}} \sum_{k} n_{k+q}\left(1-n_{k}\right) \\
& -\frac{4 U^{2}}{\Omega^{2}} \sum_{k} n_{k+q}\left(1-n_{k}\right) \int \mathrm{d} \epsilon_{1} \int \mathrm{d} \epsilon_{2^{\prime}} \int \mathrm{d} \epsilon_{2} D\left(\epsilon_{1}\right) D\left(\epsilon_{2^{\prime}}\right) D\left(\epsilon_{2}\right) \\
& \times \frac{1-\cos \left(\left(\Delta \epsilon_{k, 1,2^{\prime}, 2}\right) t\right)}{\left(\Delta \epsilon_{k, 1,2^{\prime}, 2}\right)^{2}}\left(\left(1-n_{1}\right) n_{2^{\prime}}\left(1-n_{2}\right)+n_{1}\left(1-n_{2^{\prime}}\right) n_{2}\right) \\
& +\frac{2 U^{2}}{\Omega^{4}} \sum_{k} n_{k+q}\left(1-n_{k}\right) \\
& \times \sum_{k_{i}^{\prime}, k_{i}} \frac{1-\cos \left(\left(\Delta \epsilon_{k+q, k_{1}^{\prime}, k_{2}^{\prime}, k_{2}}\right) t\right)-\cos \left(\left(\Delta \epsilon_{k, k_{1}, k_{2}^{\prime}, k_{2}}\right) t\right)+\cos \left(\left(\Delta \epsilon_{k+q, k, k_{1}, k_{1}^{\prime}}\right) t\right)}{\left(\Delta \epsilon_{k+q, k_{1}^{\prime}, k_{2}^{\prime}, k_{2}}\right)\left(\Delta \epsilon_{k, k_{1}, k_{2}^{\prime}, k_{2}}\right)} \\
& \times n_{k_{2}^{\prime}}\left(1-n_{k_{2}}\right) \delta_{k+k_{2}^{\prime}, k_{1}+k_{2}} \delta_{k_{1}^{\prime}-k_{1}, q} \\
& -\frac{2 U^{2}}{\Omega^{2}} \sum_{k} n_{k+q}\left(1-n_{k}\right) \int \mathrm{d} \epsilon_{1^{\prime}} \int \mathrm{d} \epsilon_{1} \int \mathrm{d} \epsilon_{2^{\prime}} \int \mathrm{d} \epsilon_{2} D\left(\epsilon_{1^{\prime}}\right) D\left(\epsilon_{1}\right) D\left(\epsilon_{2^{\prime}}\right) D\left(\epsilon_{2}\right) \\
& \times \frac{\Delta \epsilon_{k+q, k, 2^{\prime}, 2}}{\Delta \epsilon_{1^{\prime}, 1,2,2^{\prime}}}\left(\frac{1-\cos \left(\left(\Delta \epsilon_{k+q, k, 1^{\prime}, 1}\right) t\right)}{\left(\Delta \epsilon_{k+q, k, 2^{\prime}, 2}\right)^{2}+\left(\Delta \epsilon_{1^{\prime}, 1,2,2^{\prime}}\right)^{2}}-\frac{1-\cos \left(\left(\Delta \epsilon_{k+q, k, 2^{\prime}, 2}\right) t\right)}{\left(\Delta \epsilon_{k+q, k, 2^{\prime}, 2}\right)^{2}}\right) \\
& \times\left(n_{1^{\prime}}-n_{1}\right)\left(n_{2^{\prime}}-n_{2}\right) \\
& +\frac{2 U^{2}}{\Omega^{4}} \sum_{k}\left(n_{k+q}\left(1-n_{k}\right)+\left(1-n_{k+q}\right) n_{k}\right) \\
& \times \sum_{k_{i}^{\prime}, k_{i}} \frac{\Delta \epsilon_{k, k_{1}, k_{2}^{\prime}, k_{2}}}{\Delta \epsilon_{k+q, k_{1}^{\prime}, k_{2}^{\prime}, k_{2}}}\left(\frac{1-\cos \left(\left(\Delta \epsilon_{k+q, k, k_{1}, k_{1}^{\prime}}\right) t\right)}{\left(\Delta \epsilon_{k+q, k_{1}^{\prime}, k_{2}^{\prime}, k_{2}}\right)^{2}+\left(\Delta \epsilon_{k, k_{1}, k_{2}, k_{2}}\right)^{2}}-\frac{1-\cos \left(\left(\Delta \epsilon_{k, k_{1}, k_{2}^{\prime}, k_{2}}\right) t\right)}{\left(\Delta \epsilon_{k, k_{1}, k_{2}^{\prime}, k_{2}}\right)^{2}}\right) \\
& \times n_{k_{1}}\left(n_{k_{2}^{\prime}}-n_{k_{2}}\right) \delta_{k+k_{2}^{\prime}, k_{1}+k_{2}} \delta_{k_{1}^{\prime}-k_{1}, q} \\
& +\frac{2 U^{2}}{\Omega^{2}} \sum_{k} n_{k+q}\left(1-n_{k}\right) \int \mathrm{d} \epsilon_{1^{\prime}} \int \mathrm{d} \epsilon_{1} \int \mathrm{d} \epsilon_{2^{\prime}} \int \mathrm{d} \epsilon_{2} D\left(\epsilon_{1^{\prime}}\right) D\left(\epsilon_{1}\right) D\left(\epsilon_{2^{\prime}}\right) D\left(\epsilon_{2}\right) \\
& \times \frac{\left(\Delta \epsilon_{1^{\prime}, 1,2,2^{\prime}}\right)+\left(\Delta \epsilon_{k+q, k, 2,2^{\prime}}\right)}{\left(\Delta \epsilon_{k+q, k, 1,1^{\prime}}\right)}\left(\frac{1-\cos \left(\left(\Delta \epsilon_{k+q, k, 1,1^{\prime}}\right) t\right)}{\left(\Delta \epsilon_{1^{\prime}, 1,2,2^{\prime}}\right)^{2}+\left(\Delta \epsilon_{k+q, k, 2,2^{\prime}}\right)^{2}}\right) \\
& \times\left(n_{k_{1}^{\prime}}-n_{k_{1}}\right)\left(n_{k_{2}^{\prime}}-n_{k_{2}}\right) \\
& +\frac{2 U^{2}}{\Omega^{4}} \sum_{k}\left(n_{k+q}\left(1-n_{k}\right)+\left(1-n_{k+q}\right) n_{k}\right) \\
& \times \sum_{k_{i}^{\prime}, k_{i}} \frac{\left(\Delta \epsilon_{k+q, k_{1}^{\prime}, k_{2}^{\prime}, k_{2}}\right)+\left(\Delta \epsilon_{k, k_{1}, k_{2}^{\prime}, k_{2}}\right)}{\left(\Delta \epsilon_{k+q, k, k_{1}, k_{1}^{\prime}}\right)}\left(\frac{1-\cos \left(\left(\Delta \epsilon_{k+q, k, k_{1}, k_{1}^{\prime}}\right) t\right)}{\left(\Delta \epsilon_{k+q, k_{1}^{\prime}, k_{2}^{\prime}, k_{2}}\right)^{2}+\left(\Delta \epsilon_{k, k_{1}, k_{2}^{\prime}, k_{2}}\right)^{2}}\right) \\
& \times n_{k_{1}^{\prime}}\left(n_{k_{2}^{\prime}}-n_{k_{2}}\right) \delta_{k+k_{2}^{\prime}, k_{1}+k_{2}} \delta_{k_{1}^{\prime}-k_{1}, q}
\end{aligned}
$$




$$
\begin{aligned}
& +\frac{U^{2}}{\Omega^{2}} \sum_{k} n_{k+q}\left(1-n_{k}\right) \int \mathrm{d} \epsilon_{1^{\prime}} \int \mathrm{d} \epsilon_{1} \int \mathrm{d} \epsilon_{2^{\prime}} \int \mathrm{d} \epsilon_{2} D\left(\epsilon_{1^{\prime}}\right) D\left(\epsilon_{1}\right) D\left(\epsilon_{2^{\prime}}\right) D\left(\epsilon_{2}\right) \\
& \times \frac{1-\cos \left(\left(\Delta \epsilon_{k+q, k, 1^{\prime}, 1}\right) t\right)-\cos \left(\left(\Delta \epsilon_{k+q, k, 2^{\prime}, 2}\right) t\right)+\cos \left(\left(\Delta \epsilon_{1^{\prime}, 1,2,2^{\prime}}\right) t\right)}{\left(\Delta \epsilon_{k+q, k, 1^{\prime}, 1}\right)\left(\Delta \epsilon_{k+q, k, 2^{\prime}, 2}\right)} \\
& \times\left(n_{1^{\prime}}-n_{1}\right)\left(n_{2^{\prime}}-n_{2}\right) \\
& -\frac{2 U^{2}}{\Omega^{4}} \sum_{k} n_{k+q} \\
& \times \sum_{k_{i}^{\prime}, k_{i}} \frac{1-\cos \left(\left(\Delta \epsilon_{k+q, k_{1}^{\prime}, k_{2}^{\prime}, k_{2}}\right) t\right)-\cos \left(\left(\Delta \epsilon_{k, k_{1}, k_{2}^{\prime}, k_{2}}\right) t\right)+\cos \left(\left(\Delta \epsilon_{k+q, k, k_{1}, k_{1}^{\prime}}\right) t\right)}{\left(\Delta \epsilon_{k+q, k_{1}^{\prime}, k_{2}^{\prime}, k_{2}}\right)\left(\Delta \epsilon_{k, k_{1}, k_{2}^{\prime}, k_{2}}\right)} \\
& \times\left(1-n_{k_{1}}\right) n_{k_{2}^{\prime}}\left(1-n_{k_{2}}\right) \delta_{k+k_{2}^{\prime}, k_{1}+k_{2}} \delta_{k_{1}^{\prime}-k_{1}, q} \\
& +\frac{4 U^{2}}{\Omega^{2}} \sum_{k} n_{k+q} \int \mathrm{d} \epsilon_{1} \int \mathrm{d} \epsilon_{2^{\prime}} \int \mathrm{d} \epsilon_{2} D\left(\epsilon_{1}\right) D\left(\epsilon_{2^{\prime}}\right) D\left(\epsilon_{2}\right) \\
& \times \frac{1-\cos \left(\left(\Delta \epsilon_{k, 1,2^{\prime}, 2}\right) t\right)}{\left(\Delta \epsilon_{k, 1,2^{\prime}, 2}\right)^{2}}\left(1-n_{1}\right) n_{2^{\prime}}\left(1-n_{2}\right) \\
& +O\left(U^{3}\right) \text {. }
\end{aligned}
$$

\section{Equilibrium correlation function}

For the equilibrium correlation function, we get

$$
\begin{aligned}
& \hat{C}_{q}^{\text {eq. }} \stackrel{\uparrow}{ } \stackrel{d=\infty}{=} \frac{1}{\Omega^{2}} \sum_{k} n_{k+q}\left(1-n_{k}\right) \\
& -\frac{2 U^{2}}{\Omega^{2}} \sum_{k} n_{k+q}\left(1-n_{k}\right) \int \mathrm{d} \epsilon_{1} \int \mathrm{d} \epsilon_{2^{\prime}} \int \mathrm{d} \epsilon_{2} D\left(\epsilon_{1}\right) D\left(\epsilon_{2^{\prime}}\right) D\left(\epsilon_{2}\right) \\
& \times \frac{1}{\left(\Delta \epsilon_{k, 1,2^{\prime}, 2}\right)^{2}}\left(\left(1-n_{1}\right) n_{2^{\prime}}\left(1-n_{2}\right)+n_{1}\left(1-n_{2^{\prime}}\right) n_{2}\right) \\
& +\frac{2 U^{2}}{\Omega^{4}} \sum_{k} n_{k+q}\left(1-n_{k}\right) \\
& \times \sum_{k_{i}^{\prime}, k_{i}} \frac{1}{\left(\Delta \epsilon_{k+q, k_{1}^{\prime}, k_{2}^{\prime}, k_{2}}\right)\left(\Delta \epsilon_{k, k_{1}, k_{2}^{\prime}, k_{2}}\right)} \\
& \times n_{k_{2}^{\prime}}\left(1-n_{k_{2}}\right) \delta_{k+k_{2}^{\prime}, k_{1}+k_{2}} \delta_{k_{1}^{\prime}-k_{1}, q} \\
& -\frac{2 U^{2}}{\Omega^{2}} \sum_{k} n_{k+q}\left(1-n_{k}\right) \int \mathrm{d} \epsilon_{1^{\prime}} \int \mathrm{d} \epsilon_{1} \int \mathrm{d} \epsilon_{2^{\prime}} \int \mathrm{d} \epsilon_{2} D\left(\epsilon_{1^{\prime}}\right) D\left(\epsilon_{1}\right) D\left(\epsilon_{2^{\prime}}\right) D\left(\epsilon_{2}\right) \\
& \times \frac{\Delta \epsilon_{k+q, k, 2^{\prime}, 2}}{\Delta \epsilon_{1^{\prime}, 1,2,2^{\prime}}}\left(\frac{1}{\left(\Delta \epsilon_{k+q, k, 2^{\prime}, 2}\right)^{2}+\left(\Delta \epsilon_{1^{\prime}, 1,2,2^{\prime}}\right)^{2}}-\frac{1}{\left(\Delta \epsilon_{k+q, k, 2^{\prime}, 2}\right)^{2}}\right) \\
& \times\left(n_{1^{\prime}}-n_{1}\right)\left(n_{2^{\prime}}-n_{2}\right)
\end{aligned}
$$




$$
\begin{aligned}
& +\frac{2 U^{2}}{\Omega^{4}} \sum_{k}\left(n_{k+q}\left(1-n_{k}\right)+\left(1-n_{k+q}\right) n_{k}\right) \\
& \times \sum_{k_{i}^{\prime}, k_{i}} \frac{\Delta \epsilon_{k, k_{1}, k_{2}^{\prime}, k_{2}}}{\Delta \epsilon_{k+q, k_{1}^{\prime}, k_{2}^{\prime}, k_{2}}}\left(\frac{1}{\left(\Delta \epsilon_{k+q, k_{1}^{\prime}, k_{2}^{\prime}, k_{2}}\right)^{2}+\left(\Delta \epsilon_{k, k_{1}, k_{2}^{\prime}, k_{2}}\right)^{2}}-\frac{1}{\left(\Delta \epsilon_{k, k_{1}, k_{2}^{\prime}, k_{2}}\right)^{2}}\right) \\
& \times n_{k_{1}}\left(n_{k_{2}^{\prime}}-n_{k_{2}}\right) \delta_{k+k_{2}^{\prime}, k_{1}+k_{2}} \delta_{k_{1}^{\prime}-k_{1}, q} \\
& +\frac{2 U^{2}}{\Omega^{2}} \sum_{k} n_{k+q}\left(1-n_{k}\right) \int \mathrm{d} \epsilon_{1^{\prime}} \int \mathrm{d} \epsilon_{1} \int \mathrm{d} \epsilon_{2^{\prime}} \int \mathrm{d} \epsilon_{2} D\left(\epsilon_{1^{\prime}}\right) D\left(\epsilon_{1}\right) D\left(\epsilon_{2^{\prime}}\right) D\left(\epsilon_{2}\right) \\
& \times \frac{\left(\Delta \epsilon_{1^{\prime}, 1,2,2^{\prime}}\right)+\left(\Delta \epsilon_{k+q, k, 2,2^{\prime}}\right)}{\left(\Delta \epsilon_{k+q, k, 1,1^{\prime}}\right)}\left(\frac{1}{\left(\Delta \epsilon_{1^{\prime}, 1,2,2^{\prime}}\right)^{2}+\left(\Delta \epsilon_{k+q, k, 2,2^{\prime}}\right)^{2}}\right) \\
& \times\left(n_{k_{1}^{\prime}}-n_{k_{1}}\right)\left(n_{k_{2}^{\prime}}-n_{k_{2}}\right) \\
& +\frac{2 U^{2}}{\Omega^{4}} \sum_{k}\left(n_{k+q}\left(1-n_{k}\right)+\left(1-n_{k+q}\right) n_{k}\right) \\
& \times \sum_{k_{i}^{\prime}, k_{i}} \frac{\left(\Delta \epsilon_{k+q, k_{1}^{\prime}, k_{2}^{\prime}, k_{2}}\right)+\left(\Delta \epsilon_{k, k_{1}, k_{2}^{\prime}, k_{2}}\right)}{\left(\Delta \epsilon_{k+q, k, k_{1}, k_{1}^{\prime}}\right)}\left(\frac{1}{\left(\Delta \epsilon_{k+q, k_{1}^{\prime}, k_{2}^{\prime}, k_{2}}\right)^{2}+\left(\Delta \epsilon_{k, k_{1}, k_{2}^{\prime}, k_{2}}\right)^{2}}\right) \\
& \times n_{k_{1}^{\prime}}\left(n_{k_{2}^{\prime}}-n_{k_{2}}\right) \delta_{k+k_{2}^{\prime}, k_{1}+k_{2}} \delta_{k_{1}^{\prime}-k_{1}, q} \\
& +\frac{U^{2}}{\Omega^{2}} \sum_{k} n_{k+q}\left(1-n_{k}\right) \int \mathrm{d} \epsilon_{1^{\prime}} \int \mathrm{d} \epsilon_{1} \int \mathrm{d} \epsilon_{2^{\prime}} \int \mathrm{d} \epsilon_{2} D\left(\epsilon_{1^{\prime}}\right) D\left(\epsilon_{1}\right) D\left(\epsilon_{2^{\prime}}\right) D\left(\epsilon_{2}\right) \\
& \times \frac{1}{\left(\Delta \epsilon_{k+q, k, 1^{\prime}, 1}\right)\left(\Delta \epsilon_{k+q, k, 2^{\prime}, 2}\right)} \\
& \times\left(n_{1^{\prime}}-n_{1}\right)\left(n_{2^{\prime}}-n_{2}\right) \\
& -\frac{2 U^{2}}{\Omega^{4}} \sum_{k} n_{k+q} \\
& \times \sum_{k_{i}^{\prime}, k_{i}} \frac{1}{\left(\Delta \epsilon_{k+q, k_{1}^{\prime}, k_{2}^{\prime}, k_{2}}\right)\left(\Delta \epsilon_{k, k_{1}, k_{2}^{\prime}, k_{2}}\right)} \\
& \times\left(1-n_{k_{1}}\right) n_{k_{2}^{\prime}}\left(1-n_{k_{2}}\right) \delta_{k+k_{2}^{\prime}, k_{1}+k_{2}} \delta_{k_{1}^{\prime}-k_{1}, q} \\
& +\frac{2 U^{2}}{\Omega^{2}} \sum_{k} n_{k+q} \int \mathrm{d} \epsilon_{1} \int \mathrm{d} \epsilon_{2^{\prime}} \int \mathrm{d} \epsilon_{2} D\left(\epsilon_{1}\right) D\left(\epsilon_{2^{\prime}}\right) D\left(\epsilon_{2}\right) \\
& \times \frac{1}{\left(\Delta \epsilon_{k, 1,2^{\prime}, 2}\right)^{2}}\left(1-n_{1}\right) n_{2^{\prime}}\left(1-n_{2}\right) \\
& +O\left(U^{3}\right) \text {. }
\end{aligned}
$$

\subsubsection{Expansion for small momentum}

The final step in this section will be to show that the FOURIER transformed correlation functions from eqs. (6.41) and (6.42) are nonanalytic at zero temperature when the momentum $q$ 


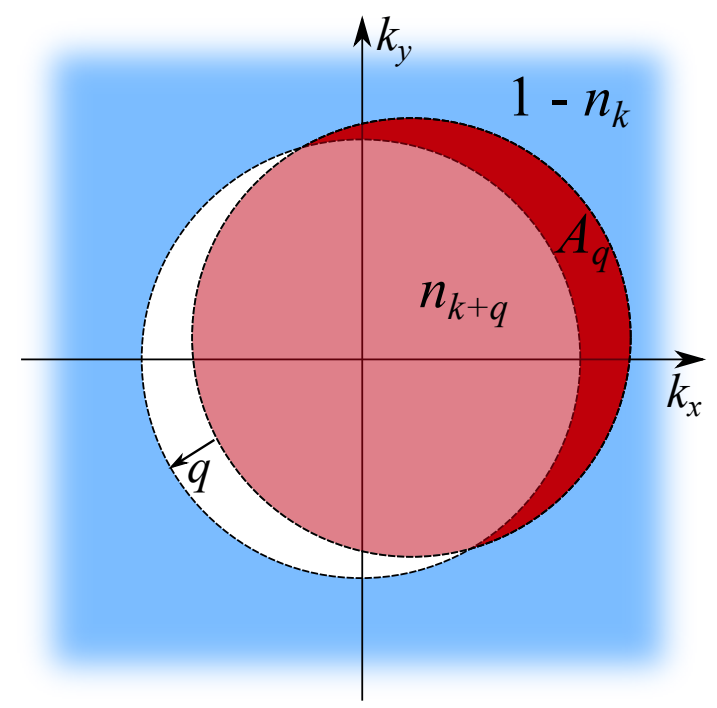

Figure 6.1: Geometrical interpretation of $A_{q}$ in $d=2$ dimensions

goes to zero in the sense that

$$
\hat{C}_{q}^{(\text {eq. }) \uparrow \uparrow}(t) \propto|q|, \quad \text { for } q \approx 0
$$

To see this, we will expand the correlation functions in orders of $q$. First, we take a closer look on the $q$-dependent term

$$
A_{q} \stackrel{\text { def }}{=} \sum_{k} n_{k+q}\left(1-n_{k}\right)
$$

For small $q$ and at zero temperature, where

$$
n_{k}= \begin{cases}0 & \text { for } \epsilon_{k}>0 \\ 1 & \text { for } \epsilon_{k}<0\end{cases}
$$

the term $A_{q}$ can be interpreted geometrically, as shown in Fig. 6.1. The dashed lines are the FERMI surface and the FERMI surface shifted by the momentum $q$ respectively. The factor $\left(1-n_{k}\right)$ is zero inside the FERMI surface and equal to 1 outside, which is indicated by the blue area. The factor $n_{k+q}$ is non-zero only inside the shifted FERMI surface, which is the red area. The dark red area is the overlap of both and hence equal to $A_{q}$ as defined above.

We see that $A_{q} \propto|q|$, at least for small $q$. So let us write

$$
A_{q} \approx c|q|
$$

with some constant $c$.

Now, we expand the FOURIER transformed correlation functions for arbitrary temperature and we find that most of the terms are at least quadratic in $q$ except for the following contributions. 


\section{Nonequilibrium correlation function}

For the nonequilibrium correlation function from eq. (6.41), we find

$$
\begin{aligned}
& \hat{C}_{q}^{\uparrow \uparrow}(t)=\frac{1}{\Omega^{2}} \sum_{k} n_{k+q}\left(1-n_{k}\right) \\
& -\frac{2 U^{2}}{\Omega^{2}} \sum_{k} n_{k+q}\left(1-n_{k}\right) \int \mathrm{d} \epsilon_{1^{\prime}} \int \mathrm{d} \epsilon_{1} \int \mathrm{d} \epsilon_{2^{\prime}} \int \mathrm{d} \epsilon_{2} D\left(\epsilon_{1^{\prime}}\right) D\left(\epsilon_{1}\right) D\left(\epsilon_{2^{\prime}}\right) D\left(\epsilon_{2}\right) \\
& \times \frac{\Delta \epsilon_{2^{\prime}, 2}}{\Delta \epsilon_{1^{\prime}, 1,2,2^{\prime}}}\left(\frac{1-\cos \left(\left(\Delta \epsilon_{1^{\prime}, 1}\right) t\right)}{\left(\Delta \epsilon_{2^{\prime}, 2}\right)^{2}+\left(\Delta \epsilon_{1^{\prime}, 1,2,2^{\prime}}\right)^{2}}-\frac{1-\cos \left(\left(\Delta \epsilon_{2^{\prime}, 2}\right) t\right)}{\left(\Delta \epsilon_{2^{\prime}, 2}\right)^{2}}\right)\left(n_{1^{\prime}}-n_{1}\right)\left(n_{2^{\prime}}-n_{2}\right) \\
& +\frac{2 U^{2}}{\Omega^{2}} \sum_{k} n_{k+q}\left(1-n_{k}\right) \int \mathrm{d} \epsilon_{1^{\prime}} \int \mathrm{d} \epsilon_{1} \int \mathrm{d} \epsilon_{2^{\prime}} \int \mathrm{d} \epsilon_{2} D\left(\epsilon_{1^{\prime}}\right) D\left(\epsilon_{1}\right) D\left(\epsilon_{2^{\prime}}\right) D\left(\epsilon_{2}\right) \\
& \times \frac{\left(\Delta \epsilon_{1^{\prime}, 1,2,2^{\prime}}\right)+\left(\Delta \epsilon_{2,2^{\prime}}\right)}{\left(\Delta \epsilon_{1,1^{\prime}}\right)}\left(\frac{1-\cos \left(\left(\Delta \epsilon_{1,1^{\prime}}\right) t\right)}{\left(\Delta \epsilon_{1^{\prime}, 1,2,2^{\prime}}\right)^{2}+\left(\Delta \epsilon_{2,2^{\prime}}\right)^{2}}\right)\left(n_{k_{1}^{\prime}}-n_{k_{1}}\right)\left(n_{k_{2}^{\prime}}-n_{k_{2}}\right) \\
& +\frac{U^{2}}{\Omega^{2}} \sum_{k} n_{k+q}\left(1-n_{k}\right) \int \mathrm{d} \epsilon_{1^{\prime}} \int \mathrm{d} \epsilon_{1} \int \mathrm{d} \epsilon_{2^{\prime}} \int \mathrm{d} \epsilon_{2} D\left(\epsilon_{1^{\prime}}\right) D\left(\epsilon_{1}\right) D\left(\epsilon_{2^{\prime}}\right) D\left(\epsilon_{2}\right) \\
& \times \frac{1-\cos \left(\left(\Delta \epsilon_{1^{\prime}, 1}\right) t\right)-\cos \left(\left(\Delta \epsilon_{2^{\prime}, 2}\right) t\right)+\cos \left(\left(\Delta \epsilon_{1^{\prime}, 1,2,2^{\prime}}\right) t\right)}{\left(\Delta \epsilon_{1^{\prime}, 1}\right)\left(\Delta \epsilon_{2^{\prime}, 2}\right)}\left(n_{1^{\prime}}-n_{1}\right)\left(n_{2^{\prime}}-n_{2}\right) \\
& +O\left(q^{2}\right)+O\left(U^{3}\right)
\end{aligned}
$$

where $\Delta \epsilon_{1^{\prime}, 1} \stackrel{\text { def }}{=} \epsilon_{1^{\prime}}-\epsilon_{1}$.

\section{Equilibrium correlation function}

For the equilibrium correlation function from eq. (6.42), we find

$$
\begin{aligned}
\hat{C}_{q}^{\text {eq } \uparrow \uparrow}= & \frac{1}{\Omega^{2}} \sum_{k} n_{k+q}\left(1-n_{k}\right) \\
- & \frac{2 U^{2}}{\Omega^{2}} \sum_{k} n_{k+q}\left(1-n_{k}\right) \int \mathrm{d} \epsilon_{1^{\prime}} \int \mathrm{d} \epsilon_{1} \int \mathrm{d} \epsilon_{2^{\prime}} \int \mathrm{d} \epsilon_{2} D\left(\epsilon_{1^{\prime}}\right) D\left(\epsilon_{1}\right) D\left(\epsilon_{2^{\prime}}\right) D\left(\epsilon_{2}\right) \\
& \times \frac{\Delta \epsilon_{2^{\prime}, 2}}{\Delta \epsilon_{1^{\prime}, 1,2,2^{\prime}}}\left(\frac{1}{\left(\Delta \epsilon_{2^{\prime}, 2}\right)^{2}+\left(\Delta \epsilon_{1^{\prime}, 1,2,2^{\prime}}\right)^{2}}-\frac{1}{\left(\Delta \epsilon_{2^{\prime}, 2}\right)^{2}}\right)\left(n_{1^{\prime}}-n_{1}\right)\left(n_{2^{\prime}}-n_{2}\right) \\
+ & \frac{2 U^{2}}{\Omega^{2}} \sum_{k} n_{k+q}\left(1-n_{k}\right) \int \mathrm{d} \epsilon_{1^{\prime}} \int \mathrm{d} \epsilon_{1} \int \mathrm{d} \epsilon_{2^{\prime}} \int \mathrm{d} \epsilon_{2} D\left(\epsilon_{1^{\prime}}\right) D\left(\epsilon_{1}\right) D\left(\epsilon_{2^{\prime}}\right) D\left(\epsilon_{2}\right) \\
& \times \frac{\left(\Delta \epsilon_{1^{\prime}, 1,2,2^{\prime}}\right)+\left(\Delta \epsilon_{2,2^{\prime}}\right)}{\left(\Delta \epsilon_{1,1^{\prime}}\right)}\left(\frac{1}{\left(\Delta \epsilon_{1^{\prime}, 1,2,2^{\prime}}\right)^{2}+\left(\Delta \epsilon_{2,2^{\prime}}\right)^{2}}\right)\left(n_{k_{1}^{\prime}}-n_{k_{1}}\right)\left(n_{k_{2}^{\prime}}-n_{k_{2}}\right) \\
+ & \frac{U^{2}}{\Omega^{2}} \sum_{k} n_{k+q}\left(1-n_{k}\right) \int \mathrm{d} \epsilon_{1^{\prime}} \int \mathrm{d} \epsilon_{1} \int \mathrm{d} \epsilon_{2^{\prime}} \int \mathrm{d} \epsilon_{2} D\left(\epsilon_{1^{\prime}}\right) D\left(\epsilon_{1}\right) D\left(\epsilon_{2^{\prime}}\right) D\left(\epsilon_{2}\right) \\
& \times \frac{1}{\left(\Delta \epsilon_{1^{\prime}, 1}\right)\left(\Delta \epsilon_{2^{\prime}, 2}\right)}\left(n_{1^{\prime}}-n_{1}\right)\left(n_{2^{\prime}}-n_{2}\right) \\
+ & O\left(q^{2}\right)+O\left(U^{3}\right) .
\end{aligned}
$$


Now, we can finally relate the two functions. As the cos-functions drop out when taking the time average, we get

$$
\begin{aligned}
\hat{C}_{q}^{\text {pre. } \uparrow \uparrow} & \equiv \overline{\hat{C}_{q}^{\uparrow \uparrow}(t)} \\
& =\hat{C}_{q}^{\text {eq. } \uparrow \uparrow}+O\left(q^{2}\right)+O\left(U^{3}\right) .
\end{aligned}
$$

Again, this cannot be the thermal value of the nonequilibrium correlation function because of the heating effect of the quench that increases the temperature in the long-time limit.

In the next chapter, we will argue that the nonanalytic behavior from eq. 6.43 means that the correlation functions decay algebraically in real space at zero temperature. With the solutions from eqs. 6.47) and (6.48) we will then be able to show the real-time evolution of the long-range correlations, i.e., the algebraically decaying tails of the correlation functions in the limit $\left|x^{\prime}-x\right| \rightarrow \infty$. 


\section{Chapter 7}

\section{Spatial decay of density-density correlations}

In this chapter, we will analyze the decay behavior of the nonequilibrium density-density correlations outside the effective light cone that is dictated by the LIEB-RoBINSON bounds (cf. sec. 4.2). The general result is depicted in Tab. 7.1, where $R=\left|x^{\prime}-x\right|$ is the spatial distance.

The susceptibilities decay faster than any algebraically decaying function outside the light cone, which is consistent with the LIEB-RoBINSON bounds. The correlation function for antiparallel spins also decays faster than algebraically, which is explained by the absence of entanglement between antiparallel spins in the initial state.

In contrast, the initial entanglement between parallel spins enhances the parallel-spin correlation function in the sense that it decays algebraically if the initial temperature is zero. At finite initial temperature, the parallel-spin correlation function decays faster than algebraically because long-range entanglement is broken by thermal fluctuations.

\begin{tabular}{l|l|l} 
& anti-parallel spins $\uparrow \downarrow$ & parallel spins $\uparrow \uparrow$ \\
\hline \hline susceptibility $\chi_{x^{\prime}, x}(t)$ & $O\left(R^{-\infty}\right)$ & $O\left(R^{-\infty}\right)$ \\
\hline correlation function $C_{x^{\prime}, x}(t)$ & $O\left(R^{-\infty}\right)$ & $O\left(R^{-2}\right)$ for $T=0$ \\
& & $O\left(R^{-\infty}\right)$ for $T>0$
\end{tabular}

Table 7.1: Spatial decay of different density-density correlations

We derive the faster-than-algebraic decay of the susceptibilities and the antiparallel-spin correlation function by performing a stationary phase approximation for large distances $R$. In sec. 7.1 we will demonstrate that it fails for regions outside an effective light cone and hence implies the fast decay.

The algebraic decay of the parallel-spin correlation function at zero temperature is obtained from the non-analyticity of the FOURIER transformed function, which we will point out in sec. 7.2. At finite temperature, the FoURIER transformed function becomes analytic again.

\subsection{Stationary phase approximation}

In this section, we will argue that the susceptibilities for antiparallel spins from eq. (6.13) and for parallel spins from eq. (6.16) as well as the equal-time correlation function for antiparallel 
spins from eq. 6.22) as functions on the spatial distance $x^{\prime}-x$ decay faster than algebraically in space outside some effective light cone, i.e., for distances $\left|x^{\prime}-x\right|>v t$, where $v$ is the maximum group velocity.

For the argument, we assume some general correlation function $C(R, t)$ depending on spatial distance $R=\left|x^{\prime}-x\right|$ and time $t$. Furthermore, we assume that it has the following structure,

$$
C(R, t)=\sum_{k^{\prime}, k} e^{i\left(k_{x}^{\prime}-k_{x}\right) R \pm i\left(\epsilon_{k^{\prime}}-\epsilon_{k}\right) t} f\left(k^{\prime}, k, t\right),
$$

where $k_{x}^{\left({ }^{\prime}\right)}$ are the x-components of the momentum vectors $k^{\left({ }^{\prime}\right)}$ and $f\left(k^{\prime}, k, t\right)$ is a smooth function of $k^{\prime}$ and $k$. In the continuum limit, we can replace the sums by integrals,

$$
C(R, t)=\frac{\Omega^{2}}{(2 \pi)^{2 d}} \int \mathrm{d} k^{\prime} \int \mathrm{d} k e^{i\left(k_{x}^{\prime}-k_{x}\right) R \pm i\left(\epsilon_{k^{\prime}}-\epsilon_{k}\right) t} f\left(k^{\prime}, k, t\right) .
$$

Now, we assume that the spatial distance $R$ is much larger than the lattice constant. As the latter is dimensionless in our case, this means that we take the limit $R \rightarrow \infty$. In this limit, the integral above can be evaluated in a stationary phase approximation [39]. In the integration, we will only get contributions in the vicinity of the stationary phases, where

$$
\begin{aligned}
\nabla_{k^{\prime}}\left(k_{x}^{\prime} R \pm \epsilon_{k^{\prime}} t\right) & =0, \\
\nabla_{k}\left(k_{x} R \pm \epsilon_{k} t\right) & =0 .
\end{aligned}
$$

These conditions are equivalent to

$$
\begin{gathered}
\mp \frac{\partial}{\partial k_{x}^{\prime}} \epsilon_{k^{\prime}}=\frac{R}{t}, \\
\mp \frac{\partial}{\partial k_{x}} \epsilon_{k}=\frac{R}{t} .
\end{gathered}
$$

Now, we assume a maximum group velocity $v$ such that

$$
\left|\frac{\partial \epsilon_{k}}{\partial k_{x}}\right| \leq v \quad \forall k
$$

This is e.g. given for the dispersion relation from eq. (3.7), where

$$
\left|\frac{\partial \epsilon_{k}}{\partial k_{x}}\right|=\left|2 \tau \sin \left(k_{x}\right)\right| \leq 2 \tau \quad \forall k .
$$

With this condition we will not find any stationary phases if the spatial distance lies outside the effective light cone, i.e., for $R>v t$. This in turn means that

$$
\forall n \in \mathbb{N}: \lim _{R \rightarrow \infty} R^{n} C(R, t)=0 \quad \text { for finite } t .
$$

In other words, the correlation function $C(R, t)$ decays faster in space than any algebraically decaying function [39], which we write symbolically as

$$
C(R, t)=O\left(R^{-\infty}\right) \quad \text { for } R>v t .
$$

In order to show now that the susceptibilities from eq. (6.13) and (6.16) and the equal-time correlation function for antiparallel spins from eq. (6.22) have such a decay behavior, we will demonstrate that they have a form like in eq. (7.1).

For this, we assume that the vector $x^{\prime}-x$ points in $x$-direction so that all expressions depend on $R=\left|x^{\prime}-x\right|$. 


\subsubsection{Susceptibilities}

Clearly, the susceptibility for antiparallel spins from eq. 6.13 is of the same form as eq. (7.1), with the amplitude

$$
f\left(k^{\prime}, k, t\right)=-i \frac{U}{\Omega^{3}}\left(n_{k^{\prime}}-n_{k}\right) \sum_{q^{\prime}, q} \frac{1-e^{-i\left(\Delta \epsilon_{k^{\prime}, k, q^{\prime}, q}\right) t}}{\Delta \epsilon_{k^{\prime}, k, q^{\prime}, q}}\left(n_{q^{\prime}}-n_{q}\right) \delta_{k^{\prime}+q^{\prime}, k+q} .
$$

At finite temperatures, the momentum distribution $n_{k}$ is analytic and so is the whole expression above. At zero temperature, the momentum distribution turns into a nonanalytic step function. However, the composition $\left(n_{k^{\prime}}-n_{k}\right)$ is analytic again in the vicinity of the stationary phase.

The same argument applies to the susceptibility for parallel spins from eq. 6.16).

\subsubsection{Correlation function}

We rewrite the equal-time correlation function for antiparallel spins from eq. 6.22 as

$$
\begin{aligned}
C_{x^{\prime}, x}^{\uparrow \downarrow}(t)= & \frac{U}{\Omega^{3}} \sum_{k^{\prime}, k} e^{i\left(k^{\prime}-k\right)\left(x^{\prime}-x\right)} e^{-i\left(\epsilon_{k^{\prime}}-\epsilon_{k}\right) t}\left(n_{k^{\prime}}-n_{k}\right) \sum_{q^{\prime}, q} \frac{e^{i\left(\epsilon_{k^{\prime}}-\epsilon_{k}\right) t}-e^{-i\left(\epsilon_{q^{\prime}}-\epsilon_{q}\right) t}}{\Delta \epsilon_{k^{\prime}, k, q^{\prime}, q}}\left(1-n_{q^{\prime}}\right) n_{q} \delta_{k^{\prime}+q^{\prime}, k+q} \\
& +\frac{U}{\Omega^{3}} \sum_{k^{\prime}, k} e^{i\left(k^{\prime}-k\right)\left(x^{\prime}-x\right)} e^{i\left(\epsilon_{k^{\prime}}-\epsilon_{k}\right) t}\left(n_{k^{\prime}}-n_{k}\right) \sum_{q^{\prime}, q} \frac{e^{-i\left(\epsilon_{k^{\prime}}-\epsilon_{k}\right) t}-e^{i\left(\epsilon_{q^{\prime}}-\epsilon_{q}\right) t}}{\Delta \epsilon_{k^{\prime}, k, q^{\prime}, q}}\left(1-n_{q^{\prime}}\right) n_{q} \delta_{k^{\prime}+q^{\prime}, k+q} \\
& +O\left(U^{2}\right) .
\end{aligned}
$$

Now, the same argument as for the susceptibilities applies.

The correlation function for parallel spins from eq. 6.24) does not show a smooth factor $\left(n_{k^{\prime}}-n_{k}\right)$, but instead a factor

$$
n_{k^{\prime}}\left(1-n_{k}\right)
$$

This is nonanalytic in the vicinity of the stationary phase at zero temperature (cf. sec. 6.5.3). In the next section, we will show that the correlation function for parallel spins decays algebraically in space.

At finite temperature, the above factor is analytic again so that the stationary phase argument applies.

\subsection{Algebraically decaying functions in FOUR IER space}

We calculated the FOURIER transformation of the parallel-spin correlation function in the limit of infinite spatial dimensions. At zero temperature, this function was expanded for 
small momentum $q$ in sec. 6.5.3, resulting in

$$
\begin{aligned}
\hat{C}_{q}^{\uparrow \uparrow}(t)= & \frac{1}{\Omega^{2}} c|q| \\
& -\frac{2 U^{2}}{\Omega^{2}} c|q| \int \mathrm{d} \epsilon_{1^{\prime}} \int \mathrm{d} \epsilon_{1} \int \mathrm{d} \epsilon_{2^{\prime}} \int \mathrm{d} \epsilon_{2} D\left(\epsilon_{1^{\prime}}\right) D\left(\epsilon_{1}\right) D\left(\epsilon_{2^{\prime}}\right) D\left(\epsilon_{2}\right) \\
& \times \frac{\Delta \epsilon_{2^{\prime}, 2}}{\Delta \epsilon_{1^{\prime}, 1,2,2^{\prime}}}\left(\frac{1-\cos \left(\left(\Delta \epsilon_{1^{\prime}, 1}\right) t\right)}{\left(\Delta \epsilon_{2^{\prime}, 2}\right)^{2}+\left(\Delta \epsilon_{1^{\prime}, 1,2,2^{\prime}}\right)^{2}}-\frac{1-\cos \left(\left(\Delta \epsilon_{2^{\prime}, 2}\right) t\right)}{\left(\Delta \epsilon_{2^{\prime}, 2}\right)^{2}}\right)\left(n_{1^{\prime}}-n_{1}\right)\left(n_{2^{\prime}}-n_{2}\right) \\
& +\frac{2 U^{2}}{\Omega^{2}} c|q| \int \mathrm{d} \epsilon_{1^{\prime}} \int \mathrm{d} \epsilon_{1} \int \mathrm{d} \epsilon_{2^{\prime}} \int \mathrm{d} \epsilon_{2} D\left(\epsilon_{1^{\prime}}\right) D\left(\epsilon_{1}\right) D\left(\epsilon_{2^{\prime}}\right) D\left(\epsilon_{2}\right) \\
& \times \frac{\left(\Delta \epsilon_{1^{\prime}, 1,2,2^{\prime}}\right)+\left(\Delta \epsilon_{2,2^{\prime}}\right)}{\left(\Delta \epsilon_{1,1^{\prime}}\right)}\left(\frac{1-\cos \left(\left(\Delta \epsilon_{1,1^{\prime}}\right) t\right)}{\left(\Delta \epsilon_{1^{\prime}, 1,2,2^{\prime}}\right)^{2}+\left(\Delta \epsilon_{2,2^{\prime}}\right)^{2}}\right)\left(n_{k_{1}^{\prime}}-n_{k_{1}}\right)\left(n_{k_{2}^{\prime}}-n_{k_{2}}\right) \\
& +\frac{U^{2}}{\Omega^{2}} c|q| \int \mathrm{d} \epsilon_{1^{\prime}} \int \mathrm{d} \epsilon_{1} \int \mathrm{d} \epsilon_{2^{\prime}} \int \mathrm{d} \epsilon_{2} D\left(\epsilon_{1^{\prime}}\right) D\left(\epsilon_{1}\right) D\left(\epsilon_{2^{\prime}}\right) D\left(\epsilon_{2}\right) \\
& \times \frac{1-\cos \left(\left(\Delta \epsilon_{1^{\prime}, 1}\right) t\right)-\cos \left(\left(\Delta \epsilon_{2^{\prime}, 2}\right) t\right)+\cos \left(\left(\Delta \epsilon_{1^{\prime}, 1,2,2^{\prime}}\right) t\right)}{\left(\Delta \epsilon_{1^{\prime}, 1}\right)\left(\Delta \epsilon_{2^{\prime}, 2}\right)}\left(n_{1^{\prime}}-n_{1}\right)\left(n_{2^{\prime}}-n_{2}\right) \\
& +O\left(q^{2}\right)+O\left(U^{3}\right) .
\end{aligned}
$$

Now, we will argue that this form in FOURIER space is equivalent to an algebraic decay for large distances $x^{\prime}-x$ in real space.

For the argument, let $C(x)$ be a general correlation function depending on distance $x$ and let

$$
\hat{C}(q) \equiv \mathcal{F}_{x}[C(x)](q)
$$

be its FOURIER transformation.

For power functions $q^{n}$, one can derive the following two rules for the inverse FouRIER transformations [14],

$$
\begin{array}{ccrl}
\mathcal{F}_{q}^{-1}\left[q^{n}\right](x) & =(i)^{n} \sqrt{2 \pi} \delta^{(n)}(x) & \forall n \in \mathbb{N}, \\
\mathcal{F}_{q}^{-1}\left[\operatorname{sgn}(q) q^{n}\right](x) & =(-i)^{n+1} n ! \sqrt{\frac{2}{\pi}} \frac{1}{x^{n+1}} & \forall n \in \mathbb{N} .
\end{array}
$$

We combine these rules to

$$
\mathcal{F}_{q}^{-1}\left[|q|^{n}\right](x)= \pm \begin{cases}\sqrt{2 \pi} \delta^{(n)}(x) & \text { for even } n \\ i n ! \sqrt{\frac{2}{\pi}} \frac{1}{x^{n+1}} & \text { for odd } n\end{cases}
$$

This provides helpful insight into the relation between the decay in real space and the analyticity around $q=0$ in FOURIER space. We can simply expand $\hat{f}(q)$ for small $q$ until we get a contribution $|q|^{n}$ with odd $n$. This corresponds then to the leading algebraic decay for large $x$. If we only get analytic contributions with $q^{n}$, the real-space function will decay faster than algebraically. 
For the parallel-spin correlation function in eq. (7.14), the leading-order contribution is proportional to $|q|$ and hence we argue that

$$
C_{x^{\prime}, x}^{\uparrow \uparrow}(t)=\frac{f^{(0)}(t)}{\left|x^{\prime}-x\right|^{2}}+U^{2} \frac{f^{(2)}(t)}{\left|x^{\prime}-x\right|^{2}}+O\left(\left|x^{\prime}-x\right|^{-3}\right)+O\left(U^{3}\right) \quad \text { for } R \rightarrow \infty,
$$

with some functions $f^{(0)}(t)$ and $f^{(2)}(t)$ that are defined by eq. 7.14 .

The time-dependent factor $f^{(2)}(t)$, i.e., the integrals in eq. 7.14$)$, can be calculated numerically, where we assume the semicircle density of states $D(\epsilon)=\left(2 \pi \tau^{2}\right)^{-1} \sqrt{4 \tau^{2}-\epsilon^{2}}$ of the BETHE lattice, for simplicity [42]. The algebraically decaying part of the correlation function is plotted in Fig. 7.1.

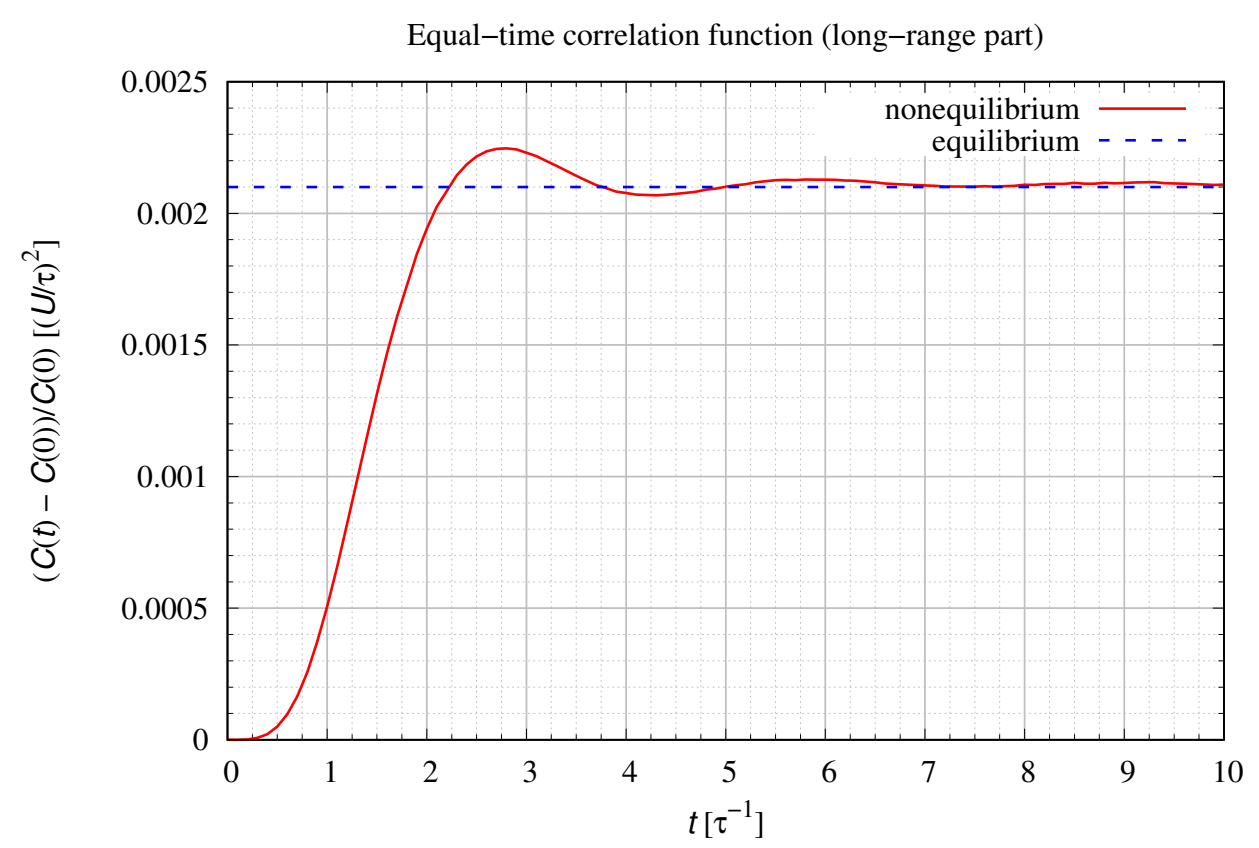

Figure 7.1: Time evolution of the quadratically decaying part of the parallel-spin correlation function $C_{x^{\prime}, x}^{\uparrow \uparrow}(t)$ at zero temperature and after subtracting initial correlations. The result is obtained by a second-order expansion in $U$. The nonequilibrium correlation function (red) reaches a prethermalization value that is equal to the equilibrium value (blue) at zero temperature.

We realize that even after subtracting the initial correlations, i.e., the $O\left(U^{0}\right)$-part, long-range correlations build up immediately after the quench. After fluctuating for a while, the longrange correlation reaches the prethermalization value at a time scale $t \sim \tau^{-1} \sim \rho_{\mathrm{F}}$. 


\section{Part IV}

\section{Discussion}





\section{Chapter 8}

\section{Conclusions and outlook}

In chapter 5 we solved the HeISENBERg equation of motion for the annihilation operator $c_{k \sigma}(t)$ in the fermionic Hubbard model in $d>1$ dimensions and with a generic dispersion relation $\epsilon_{k}$,

$$
H=\sum_{k, \sigma} \epsilon_{k} c_{k \sigma}^{\dagger} c_{k \sigma}+\frac{U}{\Omega} \sum_{k_{1}^{\prime}, k_{1}, k_{2}^{\prime}, k_{2}} c_{k_{1}^{\prime} \uparrow}^{\dagger} c_{k_{1} \uparrow} c_{k_{2}^{\prime} \downarrow}^{\dagger} c_{k_{2} \downarrow} \delta_{k_{1}^{\prime}+k_{2}^{\prime}, k_{1}+k_{2}},
$$

perturbatively in a second-order expansion for a weak interaction $U$, as compared to an average $\epsilon_{k}$. Our solution from eqs. (5.56) - (5.58) can be used to calculate expectation values with respect to the thermal state $\left|\psi_{0}\right\rangle$ of the noninteracting Hamiltonian - of any observable that is composed of products of at most four annihilation and creation operators. For observables that contain products of six or more annihilation and creation operators one would need to take into account the higher-order interaction terms in eq. 6.5).

\subsection{Reliability of the method}

We obtained our perturbative result for the time-evolved annihilation operator $c_{k \sigma}(t)$ by applying the unitary perturbation theory scheme, where the Hamiltonian and the annihilation operator are unitarily transformed into an energy-diagonal basis with the aid of the flow equation method. No secular terms appear in the result from eqs. (5.56) - (5.58).

The only approximation that we made, apart from the perturbative expansion, is the omission of elastic collision terms in the Hamiltonian,

$$
H_{\text {ela. }}=\frac{U}{\Omega} \sum_{k_{1}^{\prime}, k_{1}, k_{2}^{\prime}, k_{2}} c_{k_{1}^{\prime} \uparrow}^{\dagger} c_{k_{1} \uparrow} c_{k_{2}^{\prime} \downarrow}^{\dagger} c_{k_{2} \downarrow} \delta_{\epsilon_{k_{1}^{\prime}}^{\prime} \epsilon_{k_{2}^{\prime}}, \epsilon_{k_{1}}+\epsilon_{k_{2}}} \delta_{k_{1}^{\prime}+k_{2}^{\prime}, k_{1}+k_{2}},
$$

because they would generate secular terms in the time evolution. However, the elastic collisions only start to affect the dynamics of observables on a time scale $t \sim \rho_{\mathrm{F}}^{-3} U^{-4}$, which is reflected in the quantum BolTzMANN equation that describes the thermalization dynamics [98]. Here, $\rho_{\mathrm{F}}$ is the density of states at the Fermi level.

A time scale analysis of our perturbative result shows that it is stable for times $t \lesssim \rho_{\mathrm{F}}^{-1} U^{-2}$ in a worst case estimate. Hence, our result includes the prethermalization regime [84]. If we 
consider the dynamics of $c_{k \sigma}(t)$ at low temperature $T$ of the reference state $\left|\psi_{0}\right\rangle$ and in the vicinity of the FERmi level, $\epsilon_{k} \approx 0$, the stability will increase to times

$$
t \lesssim \min \left\{\left(\frac{1}{\rho_{\mathrm{F}}^{3} U^{2} \epsilon_{k}^{2}}\right),\left(\frac{1}{\rho_{\mathrm{F}}^{3} U^{2} T^{2}}\right)\right\} .
$$

\subsection{Calculation of density-density correlations}

In chapter 6, we used our perturbative result for $c_{k \sigma}(t)$ to derive closed expressions for densitydensity susceptibilities,

$$
\chi_{x^{\prime}, x}^{\sigma^{\prime} \sigma}(t)=-i\left\langle\left[\boldsymbol{n}_{x^{\prime}, \sigma^{\prime}}(t), \boldsymbol{n}_{x, \sigma}(0)\right]_{-}\right\rangle,
$$

and equal-time connected correlation functions,

$$
C_{x^{\prime}, x}^{\sigma^{\prime} \sigma}(t)=\left\langle\boldsymbol{n}_{x^{\prime}, \sigma^{\prime}}(t) \boldsymbol{n}_{x, \sigma}(t)\right\rangle-\left\langle\boldsymbol{n}_{x^{\prime}, \sigma^{\prime}}(t)\right\rangle\left\langle\boldsymbol{n}_{x, \sigma}(t)\right\rangle
$$

in first-order expansions for antiparallel spins, $\sigma^{\prime} \sigma=\uparrow \downarrow$, and second-order expansions for parallel spins, $\sigma^{\prime} \sigma=\uparrow \uparrow$. Here we assumed a quench setup where the initial state is the thermal state of the noninteracting Hamiltonian and the time evolution starting at $t=0$ is governed by the weakly interacting Hamiltonian.

The results from eqs. (6.13), (6.16), (6.22) and (6.24) are valid for arbitrary dispersion relations $\epsilon_{k}$, dimensions $d$ and initial temperatures $T$ on a time scale up to and including the prethermalization regime, $t \lesssim \rho_{\mathrm{F}}^{-1} U^{-2}$.

\subsection{Prethermalization of correlation functions}

In chapter 6 we also calculated time averages of the equal-time correlation functions, which are equal to their prethermalization values $C_{x^{\prime}, x}^{\text {pre. } \sigma \sigma^{\prime}}$ because our perturbative approach only covers time scales up to and including the prethermalization regime. We compared the prethermalization values of the nonequilibrium correlations with the equilibrium correlations $C_{x^{\prime}, x}^{\text {eq. } \sigma \sigma^{\prime}}$ of the interacting Hamiltonian. For the nonequilibrium functions we assumed an initial temperature $T$ of the pre-quench state, which is the thermal state of the noninteracting Hamiltonian. We assigned the same temperature $T$ to the values $C_{x^{\prime}, x}^{\text {eq. } \sigma \sigma^{\prime}}$ for the interacting HubBARD model in equilibrium.

For antiparallel spins, we found that

$$
C_{x^{\prime}, x}^{\mathrm{pre} \uparrow \downarrow}=C_{x^{\prime}, x}^{\mathrm{eq} \uparrow \downarrow}+O\left(U^{2}\right) .
$$

This means that to leading order the nonequilibrium correlation function reaches its equilibrium value at the pre-quench temperature within the prethermalization regime. However, in the long-time limit, which is not covered by our approach, we expect the heating effect of the quench to further increase the temperature.

For parallel spins, we only found that in the DMFT limit of infinite spatial dimensions

$$
\hat{C}_{q}^{\text {pre. } \uparrow \uparrow}=\hat{C}_{q}^{\text {eq. } \uparrow \uparrow}+O\left(q^{2}\right)+O\left(U^{3}\right)
$$


where $\hat{C}_{q}=\Omega^{-1} \sum_{x^{\prime}-x} e^{-i q\left(x^{\prime}-x\right)} C_{x^{\prime}, x}$ is the FOURIER transformation of the correlation function to momentum space. Hence, to leading order, the nonequilibrium correlation function for parallel spins reaches the equilibrium value in the limit of small $q$. This limit is associated with the long-range correlations in real space.

We conclude that our findings are close to the original picture of prethermalization by BERGES et al. [7], discussed in sec. 4.1. While in the prethermalization regime mode quantities like the momentum distribution from eq. (4.2) still differ from equilibrium, local quantities like the interaction energy from eq. (4.4), which is a sum over local terms, and the density-density correlation functions from eqs. (8.6) and (8.7) already prethermalize to their equilibrium values. Admittedly, these prethermal equilibrium values are not the thermal values because the heating effect of the quench will increase the temperature in the long-time limit when thermalization sets in. In accordance with the image of prethermalization plateaus as nearintegrability induced bottlenecks in the thermalization dynamics, the prethermal equilibrium values are the more robust, viz for times $t \sim \rho_{\mathrm{F}}^{-1} U^{-2}$, the closer the system is to the integrable point $U=0$.

The discussion of prethermalization of correlation functions is published at [67].

\subsection{LIEB-Robinson bounds}

In chapter 7 we analyzed the spatial decay behavior of the density-density susceptibilities and correlation functions.

Based on a stationary phase approximation, we demonstrated that

$$
\begin{array}{ll}
\chi_{x^{\prime}, x}^{\uparrow \downarrow}(t)=O\left(\left(x^{\prime}-x\right)^{-\infty}\right)+O\left(U^{2}\right) & \text { for } x^{\prime}-x>v t, \\
\chi_{x^{\prime}, x}^{\uparrow \uparrow}(t)=O\left(\left(x^{\prime}-x\right)^{-\infty}\right)+O\left(U^{3}\right) & \text { for } x^{\prime}-x>v t .
\end{array}
$$

This means that both susceptibilities decay faster than algebraically in space for distances $x^{\prime}-x$ that lie outside the effective light cone defined by the maximum group velocity

$$
v=\max _{k}\left(\left|\nabla_{k} \epsilon_{k}\right|\right)
$$

This holds for arbitrary initial temperatures and is in accordance with the LIEB-RoBinson bounds [74], discussed in sec. 4.2. The interpretation is that the linear response to a perturbation at lattice site $x$ propagates with a finite velocity $v$ through the system.

We also showed that the equal-time correlation function for antiparallel spins decays faster than algebraically outside the light cone,

$$
C_{x^{\prime}, x}^{\uparrow \downarrow}(t)=O\left(\left(x^{\prime}-x\right)^{-\infty}\right)+O\left(U^{2}\right) \quad \text { for } x^{\prime}-x>v t,
$$

again for arbitrary initial temperatures. This is consistent with the generalized LIEB-RoBinson bounds for equal-time correlation functions [12], also discussed in sec. 4.2, because there are no correlations between antiparallel spins in the initial state.

This is not the case for parallel spins at zero temperature. Though the initial state is a product state in momentum space, it is highly entangled in real space. In fact, the initial correlations 
between parallel spins are given by

$$
C_{x^{\prime}, x}^{\uparrow \uparrow}(t=0)=\frac{1}{\Omega^{2}} \sum_{k^{\prime}, k} e^{i\left(k^{\prime}-k\right)\left(x^{\prime}-x\right)} n_{k^{\prime}}\left(1-n_{k}\right) .
$$

At zero temperature, the nonanalyticity of $n_{k^{\prime}}\left(1-n_{k}\right)$ leads to an algebraic decay in real space. These algebraically decaying correlations spread to new long-range correlations that build up after the quench,

$$
C_{x^{\prime}, x}^{\uparrow \uparrow}(t)-C_{x^{\prime}, x}^{\uparrow \uparrow}(0)=O\left(\left(x^{\prime}-x\right)^{-2}\right)+O\left(U^{3}\right) \quad \text { for }\left|x^{\prime}-x\right| \rightarrow \infty .
$$

In Fig. 7.1, we visualized that at arbitrary small times after the quench new long-range correlations emerge in the system. This underpins the claim that entanglement in the initial state can enhance the spreading of correlations outside the effective light cone [59, 80], see Fig. 1.3, At finite temperature in the initial state, the factor $n_{k^{\prime}}\left(1-n_{k}\right)$ is analytic so that both initial and new correlations decay faster-than-algebraically. This coincides with the findings by Medvedyeva et al. for the Kondo model, where the initial temperature decreases the entanglement in the system [80].

\subsection{Outlook}

As pointed out before, the general solution for the time-evolved annihilation operator $c_{k \sigma}(t)$ has just opened the playground for further investigations of the HuBBard model in $d>1$ dimensions. For the setup of a weak interaction quench, one can calculate expectation values of any observable that contains products of at most four annihilation and creation operators, in expansions to second order in $U$. For example, one can directly compare susceptibilities to correlation functions for unequal times,

$$
\left\langle\left[\boldsymbol{n}_{x^{\prime}}\left(t^{\prime}\right), \boldsymbol{n}_{x}(t)\right]_{-}\right\rangle \quad \text { vs. } \quad\left\langle\left[\boldsymbol{n}_{x^{\prime}}\left(t^{\prime}\right), \boldsymbol{n}_{x}(t)\right]_{+}\right\rangle,
$$

in order to study fluctuation-dissipation relations in nonequilibrium [95]. This can serve for benchmarking against other methods if one is interested in time scales up to and including prethermalization, given by eq. (8.3). Our perturbative approach also provides a prediction for experiments with cold atoms.

A promising ansatz for a hybrid method is to combine unitary perturbation theory with numerical methods. Here, one solves the HeIsENBERg equation of motion for an operator with unitary perturbation theory and calculates the initial state with numerical DMRG-like methods. In this way one is not restricted to weak interaction quenches starting from a free system. For example, one can calculate the thermal state of the strongly interacting HuBBARD model numerically and implement a quench from strong to weak interaction by calculating expectation values of observables obtained with our approach.

In search of a full dynamical description of nonequilibrium systems, which also includes the thermalization regime, a worthwhile endeavor is investigating the connection between the flow equation method and the quantum Boltzmann equation. The flow equation method diagonalizes the Hamiltonian with a remnant

$$
H_{\text {ela. }}=\frac{U}{\Omega} \sum_{k_{1}^{\prime}, k_{1}, k_{2}^{\prime}, k_{2}} c_{k_{1}^{\prime} \uparrow}^{\dagger} c_{k_{1} \uparrow} c_{k_{2}^{\prime} \downarrow}^{\dagger} c_{k_{2} \downarrow} \delta_{\epsilon_{k_{1}^{\prime}}+\epsilon_{k_{2}^{\prime}}, \epsilon_{k_{1}}+\epsilon_{k_{2}}} \delta_{k_{1}^{\prime}+k_{2}^{\prime}, k_{1}+k_{2}}
$$


that cannot be dealt with without producing secular terms. The omission of $\boldsymbol{H}_{\text {ela. }}$ restricts the time evolution in unitary perturbation theory to times $t \lesssim \rho_{\mathrm{F}}^{-1} U^{-2}$. Interestingly, the energy-conserving contributions in $\boldsymbol{H}_{\text {ela. }}$ are exactly the same that appear in the collision term of the quantum BolTzMANN equation, which describes the thermalization dynamics that sets in at $t \sim \rho_{\mathrm{F}}^{-3} U^{-4}[98]$. 


\section{Acknowledgements}

I thank my supervisor Stefan Kehrein for supporting my studies and research throughout the years. He kindled my curiosity about many questions of condensed matter theory and always motivated me to stick to research. He not only provided excellent supervision while keeping enough space for me to develop own ideas, but he also financially supported my participation in conferences and summer schools and gave me the opportunity to intermit my $\mathrm{PhD}$ project for a research stay in South Africa and an internship in Hamburg.

I also thank Laura Covi for assuming the duties as my co-supervisor.

I greatly benefited from my research stay at the National Institute for Theoretical Physics (NITheP) in Stellenbosch, South Africa. I thank Michael Kastner for magnificient hospitality and support. The encounters with the people at NITheP fueled both my academic and personal development.

I am also extremely grateful for the support of the people of the CMT group in Göttingen. Especially, I thank Nils and Michael, my office colleagues, for cheering me up during my writing phase and Jan for his assistance in language issues.

My sincere thank goes to the Cusanuswerk that financially supported my $\mathrm{PhD}$ and offered numerous opportunities for further development also in other fields.

Last but not least, I thank Alex, my friends and above all my family for unconditional support.

\section{Funding information}

This work was supported through SFB 1073 (project B03) of the Deutsche Forschungsgemeinschaft (DFG) and the Bischöfliche Studienförderung Cusanuswerk. 
Appendix 



\section{Appendix A}

\section{Normal ordering}

As pointed out by KEHREIN [60], one needs an effective method to organize the higher-order interaction terms that appear when one works out the coupled flow equations. A convenient method is normal ordering, which was introduced by WICK in 1950 [116]. Our notation of normal ordering follows the one presented in [60].

\section{A.1 Definition for fermionic operators}

We define normal ordering as a linear operation : $:$ on an expression $O$ that depends on the fermionic annihilation and creation operators $c_{k \sigma}, c_{k \sigma}^{\dagger}$. Our reference state $\left|\psi_{0}\right\rangle$ is the thermal state of $\boldsymbol{H}_{0}=\sum_{k, \sigma} \epsilon_{k} c_{k \sigma}^{\dagger} c_{k \sigma}$. Then, we have the following fundamental contractions,

$$
\begin{aligned}
& \left\langle\psi_{0}\left|\boldsymbol{c}_{k \sigma} c_{k^{\prime} \sigma^{\prime}}\right| \psi_{0}\right\rangle=0, \\
& \left\langle\psi_{0}\left|\boldsymbol{c}_{k \sigma} \boldsymbol{c}_{k^{\prime} \sigma^{\prime}}^{\dagger}\right| \psi_{0}\right\rangle=\left(1-n_{k}\right) \delta_{k, k^{\prime}} \delta_{\sigma, \sigma^{\prime}}, \\
& \left\langle\psi_{0}\left|\boldsymbol{c}_{k \sigma}^{\dagger} \boldsymbol{c}_{k^{\prime} \sigma^{\prime}}\right| \psi_{0}\right\rangle=n_{k} \delta_{k, k^{\prime}} \delta_{\sigma, \sigma^{\prime}}, \\
& \left\langle\psi_{0}\left|\boldsymbol{c}_{k \sigma}^{\dagger} \boldsymbol{c}_{k^{\prime} \sigma^{\prime}}^{\dagger}\right| \psi_{0}\right\rangle=0,
\end{aligned}
$$

with the Fermi-Dirac distribution $n_{k}$.

Normal ordering is now defined by a recurrence relation. The inital step is just

$$
\begin{aligned}
& : c_{k \sigma}:=c_{k \sigma}, \\
& : c_{k \sigma}^{\dagger}:=c_{k \sigma}^{\dagger} .
\end{aligned}
$$

Then, for a known normal-ordered expression : $O$ : we can recursively define

$$
\begin{aligned}
& c_{k \sigma}: O:=: c_{k \sigma} O:+\left(1-n_{k}\right): \frac{\partial O}{\partial c_{k \sigma}^{\dagger}}:, \\
& c_{k \sigma}^{\dagger}: O:=: c_{k \sigma}^{\dagger} O:+n_{k}: \frac{\partial O}{\partial c_{k \sigma}}: .
\end{aligned}
$$

When taking the derivatives, it is important to bear in mind the following rule for fermionic operators $A_{k}, A_{l}$,

$$
\frac{\partial}{\partial A_{l}} A_{k}=\delta_{k l}-A_{k} \frac{\partial}{\partial A_{l}}
$$


The fermionic anticommutativity also yields minus sign, whenever we exchange two operators in normal-ordered expression, i.e.,

$$
: \ldots A_{k} A_{l} \ldots:=-: \ldots A_{l} A_{k} \ldots: \text {. }
$$

\section{A.2 Normal-ordered expressions}

Here, we write down two examples for normal-ordered expressions that are obtained by the recurrence relation from eqs. (A.7) and A.8.).

1. One-particle interaction:

$$
\begin{aligned}
c_{k_{1}^{\prime} \sigma}^{\dagger} c_{k_{1} \sigma} & =c_{k_{1}^{\prime} \sigma}^{\dagger}: c_{k_{1} \sigma}: \\
& =: c_{k_{1}^{\prime} \sigma}^{\dagger} c_{k_{1} \sigma}:+n_{k_{1}^{\prime}} s_{k_{1}^{\prime}, k_{1}} .
\end{aligned}
$$

2. Two-particle interaction:

$$
\begin{aligned}
c_{k_{1}^{\prime} \uparrow}^{\dagger} c_{k_{1} \uparrow} c_{k_{2}^{\prime} \downarrow}^{\dagger} c_{k_{2} \downarrow}= & c_{k_{1}^{\prime} \uparrow}^{\dagger} c_{k_{1} \uparrow} c_{k_{2}^{\prime} \downarrow}^{\dagger}: c_{k_{2} \downarrow}: \\
= & c_{k_{1}^{\prime} \uparrow}^{\dagger} c_{k_{1} \uparrow}: c_{k_{2}^{\prime} \downarrow}^{\dagger} c_{k_{2} \downarrow}:+c_{k_{1}^{\prime} \uparrow}^{\dagger} c_{k_{1} \uparrow} n_{k_{2}^{\prime}} \delta_{k_{2}, k_{2}} \\
= & c_{k_{1}^{\prime} \uparrow}^{\dagger}: c_{k_{1} \uparrow} c_{k_{2}^{\prime} \downarrow}^{\dagger} c_{k_{2} \downarrow}:+: c_{k_{1}^{\prime} \uparrow}^{\dagger} c_{k_{1} \uparrow}: n_{k_{2}^{\prime}} \delta_{k_{2}^{\prime}, k_{2}} \\
& +n_{k_{1}^{\prime}} \delta_{k_{1}^{\prime}, k_{1}} n_{k_{2}^{\prime}} \delta_{k_{2}^{\prime}, k_{2}} \\
= & : c_{k_{1}^{\prime} \uparrow}^{\dagger} c_{k_{1} \uparrow} c_{k_{2}^{\prime} \downarrow}^{\dagger} c_{k_{2} \downarrow}:+: c_{k_{2}^{\prime} \downarrow}^{\dagger} c_{k_{2} \downarrow}: n_{k_{1}^{\prime}} \delta_{k_{1}^{\prime}, k_{1}} \\
& +: c_{k_{1}^{\prime} \uparrow}^{\dagger} c_{k_{1} \uparrow}: n_{k_{2}^{\prime}} \delta_{k_{2}, k_{2}}+n_{k_{1}^{\prime}} \delta_{k_{1}^{\prime}, k_{1}} n_{k_{2}^{\prime}} \delta_{k_{2}, k_{2}} .
\end{aligned}
$$

Now, we immediately see that normal ordering of the Hamiltonian from eq. (3.10), i.e.,

$$
\begin{aligned}
H= & \sum_{k, \sigma} \epsilon_{k} c_{k \sigma}^{\dagger} c_{k \sigma}+\frac{U}{\Omega} \sum_{k_{1}^{\prime}, k_{1}, k_{2}^{\prime}, k_{2}} c_{k_{1}^{\prime} \uparrow}^{\dagger} c_{k_{1} \uparrow} c_{k_{2}^{\prime} \downarrow}^{\dagger} c_{k_{2} \downarrow} \delta_{k_{1}^{\prime}+k_{2}^{\prime}, k_{1}+k_{2}} \\
= & \sum_{k, \sigma}\left(\epsilon_{k}+\frac{U}{2}\right): c_{k \sigma}^{\dagger} c_{k \sigma}:+\frac{U}{\Omega} \sum_{k_{1}^{\prime}, k_{1}, k_{2}^{\prime}, k_{2}}: c_{k_{1}^{\prime} \uparrow}^{\dagger} c_{k_{1} \uparrow} c_{k_{2}^{\prime} \downarrow}^{\dagger} c_{k_{2} \downarrow}: \delta_{k_{1}^{\prime}+k_{2}^{\prime}, k_{1}+k_{2}} \\
& +2 \sum_{k} n_{k} \epsilon_{k}+\frac{U \Omega}{4}
\end{aligned}
$$

only shifts the ground state energy and does not affect the dynamics.

\section{A.3 Products of normal-ordered expressions}

In the flow equation analysis, one encounters products of normal-ordered expressions, : $O_{1}::$ $\mathrm{O}_{2}$ :. Here, one can apply Wick's second theorem [60, 116], which can be written as

$$
: \boldsymbol{O}_{1}:: \boldsymbol{O}_{2}:=: \exp \left(\sum_{k, l} C_{k l} \frac{\partial^{2}}{\partial A_{l}^{\prime} \partial A_{k}}\right) O_{1}(A) \boldsymbol{O}_{2}\left(A^{\prime}\right):\left.\right|_{A^{\prime}=A},
$$


where $C_{k l}=\left\langle\psi_{0}\left|A_{k} A_{l}\right| \psi_{0}\right\rangle$ is the contraction of $A_{k}$ and $A_{l}$. For example, we get

$$
\begin{aligned}
: c_{k_{1}^{\prime} \sigma}^{\dagger} c_{k_{1} \sigma}:: c_{k_{2}^{\prime} \sigma}^{\dagger} c_{k_{2} \sigma}:= & : c_{k_{1}^{\prime} \sigma}^{\dagger} c_{k_{1} \sigma} c_{k_{2}^{\prime} \sigma}^{\dagger} c_{k_{2} \sigma}:+: c_{k_{1} \sigma} c_{k_{2}^{\prime} \sigma}^{\dagger}: n_{k_{1}^{\prime}} \delta_{k_{1}^{\prime}, k_{2}} \\
& +: c_{k_{1}^{\prime} \sigma}^{\dagger} c_{k_{2} \sigma}:\left(1-n_{k_{1}}\right) \delta_{k_{1}, k_{2}^{\prime}}+n_{k_{1}^{\prime}} \delta_{k_{1}^{\prime}, k_{2}}\left(1-n_{k_{1}}\right) \delta_{k_{1}, k_{2}^{\prime}} .
\end{aligned}
$$

\section{A.4 Expectation values of normal-ordered expressions}

The main advantage of normal ordering is that expectation values of normal-ordered expressions will vanish if the expectation value is taken with respect to the reference state $\left|\psi_{0}\right\rangle$, i.e.,

$$
\left\langle\psi_{0}|: O:| \psi_{0}\right\rangle=0
$$

This will hold if the reference state is an eigenstate or thermal state of a bilinear Hamiltonian [60], which is the case here.

When calculating the expectation value of a product of two normal ordered expressions $O_{1}$ and $\mathrm{O}_{2}$, i.e.,

$$
\left\langle\psi_{0}\left|: O_{1}:: O_{2}:\right| \psi_{0}\right\rangle
$$

we use the fact that the above expression is only non-zero when $O_{1}$ and $O_{2}$ share summands with the same amount of annihilation and creation operators because otherwise we do not have full contractions after applying WicK's second theorem. In other words, the above expression can be easily evaluated by only calculating the full contractions between $\mathrm{O}_{1}$ and $\mathrm{O}_{2}$. For example, we have

$$
\begin{aligned}
\left\langle\psi_{0}\left|: c_{k_{1}^{\prime} \sigma}^{\dagger} c_{k_{1} \sigma}:: c_{k_{2}^{\prime} \sigma}^{\dagger} c_{k_{2} \sigma}:\right| \psi_{0}\right\rangle= & \left\langle\psi_{0}\left|: c_{k_{1}^{\prime} \sigma}^{\dagger} c_{k_{1} \sigma} c_{k_{2}^{\prime} \sigma}^{\dagger} c_{k_{2} \sigma}:\right| \psi_{0}\right\rangle+\left\langle\psi_{0}\left|: c_{k_{1} \sigma} c_{k_{2}^{\prime} \sigma}^{\dagger}:\right| \psi_{0}\right\rangle n_{k_{1}^{\prime}} \delta_{k_{1}^{\prime}, k_{2}} \\
& +\left\langle\psi_{0}\left|: c_{k_{1}^{\prime} \sigma}^{\dagger} c_{k_{2} \sigma}:\right| \psi_{0}\right\rangle\left(1-n_{k_{1}}\right) \delta_{k_{1}, k_{2}^{\prime}}+n_{k_{1}^{\prime}} \delta_{k_{1}^{\prime}, k_{2}}\left(1-n_{k_{1}}\right) \delta_{k_{1}, k_{2}^{\prime}} \\
= & n_{k_{1}^{\prime}} \delta_{k_{1}^{\prime}, k_{2}}\left(1-n_{k_{1}}\right) \delta_{k_{1}, k_{2}^{\prime}} .
\end{aligned}
$$




\section{Appendix B}

\section{Consistency checks}

\section{B.1 Preservation of the canonical anticommutation relation}

The application of the forward-backward scheme, depicted in Fig. 2.3, on the annihilation and creation operators $c_{k \sigma}$ and $c_{k \sigma}^{\dagger}$ is a sequence of unitary transformations. Hence, we expect the canonical anticommutation relation

$$
\left[c_{k \uparrow}(t), c_{k^{\prime} \uparrow}^{\dagger}(t)\right]_{+} \stackrel{!}{=} \delta_{k, k^{\prime}}+O\left(U^{3}\right)
$$

to be preserved at least up to second order in $U$. This motivates a consistency check for the time-evolved solutions from eqs. (5.56) - (5.58) after the unitary perturbation theory scheme has been applied to the annihilation operator. With its general form we get

$$
\begin{aligned}
{\left[c_{k \uparrow}(t), c_{k^{\prime} \uparrow}^{\dagger}(t)\right]_{+}=} & h_{k}(t) h_{k^{\prime}}^{*}(t)\left[: c_{k \uparrow}:: c_{k^{\prime} \uparrow}^{\dagger}:\right]_{+} \\
& +\sum_{k_{i}^{\prime}, k_{i}} F_{k, k_{1}, k_{2}^{\prime}, k_{2}}(t) F_{k^{\prime}, k_{3}, k_{4}^{\prime}, k_{4}}^{*}(t)\left[: c_{k_{1} \uparrow} c_{k_{2}^{\prime} \downarrow}^{\dagger} c_{k_{2} \downarrow}:: c_{k_{4} \downarrow}^{\dagger} c_{k_{4}^{\prime} \downarrow} c_{k_{3} \uparrow}^{\dagger}:\right]_{+} \\
& \times \delta_{k+k_{2}^{\prime}, k_{1}+k_{2}} \delta_{k^{\prime}+k_{4}^{\prime}, k_{3}+k_{4}} \\
& +\sum_{k_{i}^{\prime}, k_{i}} h_{k}(t) F_{k^{\prime}, k_{1}, k_{2}^{\prime}, k_{2}}^{*}(t)\left[: c_{k \uparrow}::: c_{k_{2} \downarrow}^{\dagger} c_{k_{2}^{\prime} \downarrow} c_{k_{1} \uparrow}^{\dagger}:\right]_{+} \delta_{k^{\prime}+k_{2}^{\prime}, k_{1}+k_{2}} \\
& +\sum_{k_{i}^{\prime}, k_{i}} h_{k^{\prime}}^{*}(t) F_{k, k_{1}, k_{2}^{\prime}, k_{2}}(t)\left[: c_{k_{1} \uparrow} c_{k_{2} \downarrow}^{\dagger} c_{k_{2} \downarrow}::: c_{k^{\prime} \uparrow}^{\dagger}:\right]_{+} \delta_{k+k_{2}^{\prime}, k_{1}+k_{2}} \\
& +\sum_{k_{i}^{\prime}, k_{i}} h_{k}(t) G_{k^{\prime}, k_{1}, k_{2}^{\prime}, k_{2}}^{*}(t)\left[: c_{k \uparrow}::: c_{k_{2} \uparrow}^{\dagger} c_{k_{2}^{\prime} \uparrow} c_{k_{1} \uparrow}^{\dagger}:\right]_{+} \delta_{k^{\prime}+k_{2}^{\prime}, k_{1}+k_{2}} \\
& +\sum_{k_{i}^{\prime}, k_{i}} h_{k^{\prime}}^{*}(t) G_{k, k_{1}, k_{2}, k_{2}}(t)\left[: c_{k_{1} \uparrow} c_{k_{2}^{\prime} \uparrow}^{\dagger} c_{k_{2} \uparrow}::: c_{k^{\prime} \uparrow}^{\dagger}:\right]_{+} \delta_{k+k_{2}^{\prime}, k_{1}+k_{2}} \\
& +O\left(U^{3}\right) .
\end{aligned}
$$

Now, we calculate the products of the normal-ordered expressions, where we only consider terms that have an operator structure proportional to 1 or $: c_{k_{1}^{\prime} \uparrow}^{\dagger} c_{k_{1} \uparrow}:$ after the contractions. 
This yields

$$
\begin{aligned}
{\left[c_{k \uparrow}(t), c_{k^{\prime} \uparrow}^{\dagger}(t)\right]_{+}=} & h_{k}(t) h_{k^{\prime}}^{*}(t) \delta_{k, k^{\prime}} \\
& +\sum_{k_{1}, k_{2}^{\prime}, k_{2}} F_{k, k_{1}, k_{2}^{\prime}, k_{2}}(t) F_{k^{\prime}, k_{1}, k_{2}^{\prime}, k_{2}}^{*}(t)\left(\left(1-n_{k_{1}}\right) n_{k_{2}^{\prime}}\left(1-n_{k_{2}}\right)+n_{k_{1}}\left(1-n_{k_{2}^{\prime}}\right) n_{k_{2}}\right) \\
& \times \delta_{k+k_{2}^{\prime}, k_{1}+k_{2}} \delta_{k, k^{\prime}} \\
& -\sum_{k_{i}^{\prime}, k_{i}} F_{k, k_{1}, k_{2}^{\prime}, k_{2}}(t) F_{k^{\prime}, k_{1}^{\prime}, k_{2}^{\prime}, k_{2}}^{*}(t)\left(n_{k_{2}^{\prime}}-n_{k_{2}}\right): c_{k_{1}^{\prime} \uparrow}^{\dagger} c_{k_{1} \uparrow}: \delta_{k+k_{2}^{\prime}, k_{1}+k_{2}} \delta_{k^{\prime}+k_{1}, k+k_{1}^{\prime}} \\
& +\sum_{k_{1}^{\prime}, k_{1}} h_{k}(t)\left(G_{k^{\prime}, k, k_{1}, k_{1}^{\prime}}^{*}(t)-G_{k^{\prime}, k_{1}^{\prime}, k_{1}, k}^{*}(t)\right): c_{k_{1}^{\prime} \uparrow}^{\dagger} c_{k_{1} \uparrow}: \delta_{k^{\prime}+k_{1}, k+k_{1}^{\prime}} \\
& +\sum_{k_{1}^{\prime}, k_{1}} h_{k^{\prime}}^{*}(t)\left(G_{k, k^{\prime}, k_{1}^{\prime}, k_{1}}(t)-G_{k^{\prime}, k_{1}, k_{1}^{\prime}, k^{\prime}}(t)\right): c_{k_{1}^{\prime} \uparrow}^{\dagger} c_{k_{1} \uparrow}: \delta_{k^{\prime}+k_{1}, k+k_{1}^{\prime}} \\
& + \text { linearly independent terms } \\
& +O\left(U^{3}\right) .
\end{aligned}
$$

Thus, we have two consistency conditions,

$$
\begin{aligned}
1 \stackrel{!}{=} & \left|h_{k}(t)\right|^{2} \\
& +\sum_{k_{1}, k_{2}^{\prime}, k_{2}}\left|F_{k, k_{1}, k_{2}^{\prime}, k_{2}}(t)\right|^{2}\left(\left(1-n_{k_{1}}\right) n_{k_{2}^{\prime}}\left(1-n_{k_{2}}\right)+n_{k_{1}}\left(1-n_{k_{2}^{\prime}}\right) n_{k_{2}}\right) \delta_{k+k_{2}^{\prime}, k_{1}+k_{2}} \\
& +O\left(U^{3}\right) \\
O \stackrel{!}{=} & -\sum_{k_{2}^{\prime}, k_{2}}\left|F_{k, k_{1}, k_{2}^{\prime}, k_{2}}(t)\right|^{2}\left(n_{k_{2}^{\prime}}-n_{k_{2}}\right) \delta_{k+k_{2}^{\prime}, k_{1}+k_{2}} \\
& +h_{k}(t)\left(G_{k, k_{,}, k_{1}}^{*}(t)-G_{k, k_{1}, k_{1}, k}^{*}(t)\right) \\
& +h_{k}^{*}(t)\left(G_{k, k, k_{1}, k_{1}}(t)-G_{k, k_{1}, k_{1}, k}(t)\right) \\
& +O\left(U^{3}\right)
\end{aligned}
$$

which are two relations that our three solutions from eqs. 5.56$)-(5.58)$ must fulfill. 
We insert the solutions into the first relation and get

$$
\begin{aligned}
& 1 \stackrel{!}{=} 1 \\
& -\frac{U^{2}}{\Omega^{2}} \sum_{k_{1}, k_{2}^{\prime}, k_{2}} \frac{1-e^{-i\left(\Delta \epsilon_{k, k_{1}, k_{2}^{\prime}, k_{2}}\right) t}}{\left(\Delta \epsilon_{k, k_{1}, k_{2}^{\prime}, k_{2}}\right)^{2}} \\
& \times\left(\left(1-n_{k_{1}}\right) n_{k_{2}^{\prime}}\left(1-n_{k_{2}}\right)+n_{k_{1}}\left(1-n_{k_{2}^{\prime}}\right) n_{k_{2}}\right) \delta_{k+k_{2}^{\prime}, k_{1}+k_{2}} \\
& -\frac{U^{2}}{\Omega^{2}} \sum_{k_{1}, k_{2}^{\prime}, k_{2}} \frac{1-e^{i\left(\Delta \epsilon_{k, k_{1}, k_{2}^{\prime}, k_{2}}\right) t}}{\left(\Delta \epsilon_{k, k_{1}, k_{2}^{\prime}, k_{2}}\right)^{2}} \\
& \times\left(\left(1-n_{k_{1}}\right) n_{k_{2}^{\prime}}\left(1-n_{k_{2}}\right)+n_{k_{1}}\left(1-n_{k_{2}^{\prime}}\right) n_{k_{2}}\right) \delta_{k+k_{2}^{\prime}, k_{1}+k_{2}} \\
& +\frac{U^{2}}{\Omega^{2}} \sum_{k_{1}, k_{2}^{\prime}, k_{2}} \frac{\left|1-e^{i\left(\Delta \epsilon_{k, k_{1}, k_{2}^{\prime}, k_{2}}\right) t}\right|^{2}}{\left(\Delta \epsilon_{k, k_{1}, k_{2}^{\prime}, k_{2}}\right)^{2}}\left(\left(1-n_{k_{1}}\right) n_{k_{2}^{\prime}}\left(1-n_{k_{2}}\right)+n_{k_{1}}\left(1-n_{k_{2}^{\prime}}\right) n_{k_{2}}\right) \delta_{k+k_{2}^{\prime}, k_{1}+k_{2}} \\
& +O\left(U^{3}\right) \text {. }
\end{aligned}
$$

We recognize that the last three terms cancel out. Thus, the first consistency condition is fulfilled.

The second relation requires

$$
\begin{aligned}
0 \stackrel{!}{=} & -\frac{U^{2}}{\Omega^{2}} \sum_{k_{2}^{\prime}, k_{2}} \frac{\left|1-e^{i\left(\Delta \epsilon_{k, k_{1}, k_{2}^{\prime}, k_{2}}\right) t}\right|^{2}}{\left(\Delta \epsilon_{k, k_{1}, k_{2}^{\prime}, k_{2}}\right)^{2}}\left(n_{k_{2}^{\prime}}-n_{k_{2}}\right) \delta_{k+k_{2}^{\prime}, k_{1}+k_{2}} \\
& -G_{k, k_{1}, k_{1}, k}^{*}(t) \\
& -G_{k, k_{1}, k_{1}, k}(t) \\
& +O\left(U^{3}\right)
\end{aligned}
$$

where we have used that $G_{k, k, k_{1}, k_{1}}(t)=0+O\left(U^{3}\right)$. Furthermore, eq. 5.58) implies

$$
\begin{aligned}
G_{k, k_{1}, k_{1}, k}(t)= & -\frac{U^{2}}{\Omega^{2}} \sum_{k_{3}^{\prime}, k_{3}} \frac{\Delta \epsilon_{k_{3}^{\prime}, k_{3}, k_{1}, k}}{\Delta \epsilon_{k, k_{1}, k_{3}, k_{3}^{\prime}}}\left(\frac{1-e^{i\left(\Delta \epsilon_{k, k_{1}, k_{1}, k}\right) t}}{\left(\Delta \epsilon_{k_{3}^{\prime}, k_{3}, k_{1}, k}\right)^{2}+\left(\Delta \epsilon_{k, k_{1}, k_{3}, k_{3}^{\prime}}\right)^{2}}\right. \\
& \left.\times \frac{e^{i\left(\Delta \epsilon_{k, k_{1}, k_{3}, k_{3}^{\prime}}\right) t}-e^{i\left(\Delta \epsilon_{k, k_{1}, k_{1}, k}\right) t}}{\left(\Delta \epsilon_{k_{3}^{\prime}, k_{3}, k_{1}, k}\right)^{2}}\right) \\
& +O\left(n_{k_{3}^{\prime}}-n_{k_{3}}\right) \delta_{k_{1}+k_{3}^{\prime}, k+k_{3}} \\
= & -\frac{U^{2}}{\Omega^{2}} \sum_{k_{2}^{\prime}, k_{2}} \frac{1-e^{i\left(\Delta \epsilon_{k, k_{1}, k_{2}^{\prime}, k_{2}}\right) t}}{\left(\Delta \epsilon_{k, k_{1}, k_{2}^{\prime}, k_{2}}\right)^{2}}\left(n_{k_{2}^{\prime}}-n_{k_{2}}\right) \delta_{k+k_{2}^{\prime}, k_{1}+k_{2}} \\
& +O\left(U^{3}\right) .
\end{aligned}
$$


With this result, the second relation reads

$$
\begin{aligned}
0 \stackrel{!}{=} & -\frac{U^{2}}{\Omega^{2}} \sum_{k_{2}^{\prime}, k_{2}} \frac{\left|1-e^{i\left(\Delta \epsilon_{k, k_{1}, k_{2}^{\prime}, k_{2}}\right) t}\right|^{2}}{\left(\Delta \epsilon_{k, k_{1}, k_{2}^{\prime}, k_{2}}\right)^{2}}\left(n_{k_{2}^{\prime}}-n_{k_{2}}\right) \delta_{k+k_{2}^{\prime}, k_{1}+k_{2}} \\
& +\frac{U^{2}}{\Omega^{2}} \sum_{k_{2}^{\prime}, k_{2}} \frac{1-e^{-i\left(\Delta \epsilon_{k, k_{1}, k_{2}^{\prime}, k_{2}}\right) t}}{\left(\Delta \epsilon_{k, k_{1}, k_{2}^{\prime}, k_{2}}\right)^{2}}\left(n_{k_{2}^{\prime}}-n_{k_{2}}\right) \delta_{k+k_{2}^{\prime}, k_{1}+k_{2}} \\
& +\frac{U^{2}}{\Omega^{2}} \sum_{k_{2}^{\prime}, k_{2}} \frac{1-e^{i\left(\Delta \epsilon_{k, k_{1}, k_{2}^{\prime}, k_{2}}\right) t}}{\left(\Delta \epsilon_{k, k_{1}, k_{2}^{\prime}, k_{2}}\right)^{2}}\left(n_{k_{2}^{\prime}}-n_{k_{2}}\right) \delta_{k+k_{2}^{\prime}, k_{1}+k_{2}} \\
& +O\left(U^{3}\right)
\end{aligned}
$$

which is clearly fulfilled. 


\section{B.2 Total spin-up particle number}

The total spin-up particle number

$$
N_{\uparrow} \stackrel{\text { def }}{=} \sum_{k} c_{k \uparrow}^{\dagger} c_{k \uparrow} \equiv \sum_{i} C_{i \uparrow}^{\dagger} C_{i \uparrow}
$$

is a conserved quantity in the HUBBARD model because it commutes with the Hamiltonian from eq. 3.10,

$$
\begin{aligned}
{\left[H_{0}, N_{\uparrow}\right]_{-} } & =\sum_{k, \sigma} \sum_{k^{\prime}} \epsilon_{k}\left[c_{k \sigma}^{\dagger} c_{k \sigma}, c_{k^{\prime} \uparrow}^{\dagger} c_{k^{\prime} \uparrow}\right]_{-} \\
& =0, \\
{\left[H_{\text {int }}, N_{\uparrow}\right]_{-} } & =U \sum_{i} \sum_{j}\left[C_{i \uparrow}^{\dagger} C_{i \uparrow} C_{i \downarrow}^{\dagger} C_{i \downarrow}, C_{j \uparrow}^{\dagger} C_{j \uparrow}\right]_{-} \\
& =0 .
\end{aligned}
$$

This provides a consistency check for eq. (6.7) because we can directly derive the time-evolved total spin-up particle number operator, where we only focus on terms with an operator structure proportional to 1 or : $c_{k_{1}^{\prime} \uparrow}^{\dagger} c_{k_{1} \uparrow}$ :,

$$
\begin{aligned}
N_{\uparrow}(t)= & \sum_{k}\left|h_{k}(t)\right|^{2} n_{k}+\sum_{k, k_{1}, k_{2}^{\prime}, k_{2}}\left|F_{k, k_{1}, k_{2}^{\prime}, k_{2}}(t)\right|^{2} n_{k_{1}}\left(1-n_{k_{2}^{\prime}}\right) n_{k_{2}} \delta_{k+k_{2}^{\prime}, k_{1}+k_{2}} \\
& +\sum_{k_{1}}\left|h_{k_{1}}(t)\right|^{2}: c_{k_{1} \uparrow}^{\dagger} c_{k_{1} \uparrow}: \\
& +\sum_{k_{1}^{\prime}, k_{1}, k_{2}^{\prime}, k_{2}}\left|F_{k_{1}^{\prime}, k_{1}, k_{2}^{\prime}, k_{2}}(t)\right|^{2}\left(1-n_{k_{2}^{\prime}}\right) n_{k_{2}}: c_{k_{1} \uparrow}^{\dagger} c_{k_{1} \uparrow}: \delta_{k_{1}^{\prime}+k_{2}^{\prime}, k_{1}+k_{2}} \\
& +\sum_{k_{1}^{\prime}, k_{1}} h_{k_{1}^{\prime}}^{*}(t)\left(G_{k_{1}^{\prime}, k_{1}^{\prime}, k_{1}, k_{1}}(t)-G_{k_{1}^{\prime}, k_{1}, k_{1}, k_{1}^{\prime}}(t)\right) n_{k_{1}^{\prime}}: c_{k_{1} \uparrow}^{\dagger} c_{k_{1} \uparrow}: \\
& +\sum_{k_{1}^{\prime}, k_{1}} h_{k_{1}^{\prime}}(t)\left(G_{k_{1}^{\prime}, k_{1}^{\prime}, k_{1}, k_{1}}^{*}(t)-G_{k_{1}^{\prime}, k_{1}, k_{1}, k_{1}^{\prime}}^{*}(t)\right) n_{k_{1}^{\prime}}: c_{k_{1} \uparrow}^{\dagger} c_{k_{1} \uparrow}: \\
& + \text { linearly independent terms } \\
& +O\left(U^{3}\right) .
\end{aligned}
$$


Now, we insert the expressions from eqs. 5.56 - 5.58 and get

$$
\begin{aligned}
& N_{\uparrow}(t)=\sum_{k} n_{k}-\frac{2 U^{2}}{\Omega^{2}} \sum_{k, k_{1}, k_{2}^{\prime}, k_{2}} \frac{1-\cos \left(\left(\Delta \epsilon_{k, k_{1}, k_{2}^{\prime}, k_{2}}\right) t\right)}{\left(\Delta \epsilon_{k, k_{1}, k_{2}^{\prime}, k_{2}}\right)^{2}} \\
& \times n_{k}\left(\left(1-n_{k_{1}}\right) n_{k_{2}^{\prime}}\left(1-n_{k_{2}}\right)+n_{k_{1}}\left(1-n_{k_{2}^{\prime}}\right) n_{k_{2}}\right) \delta_{k+k_{2}^{\prime}, k_{1}+k_{2}} \\
& +\frac{2 U^{2}}{\Omega^{2}} \sum_{k, k_{1}, k_{2}^{\prime}, k_{2}} \frac{1-\cos \left(\left(\Delta \epsilon_{k, k_{1}, k_{2}^{\prime}, k_{2}}\right) t\right)}{\left(\Delta \epsilon_{k, k_{1}, k_{2}^{\prime}, k_{2}}\right)^{2}} n_{k_{1}}\left(1-n_{k_{2}^{\prime}}\right) n_{k_{2}} \delta_{k+k_{2}^{\prime}, k_{1}+k_{2}} \\
& +\sum_{k_{1}}: c_{k_{1} \uparrow}^{\dagger} c_{k_{1} \uparrow}: \\
& -\frac{2 U^{2}}{\Omega^{2}} \sum_{k_{1}^{\prime}, k_{1}, k_{2}^{\prime}, k_{2}} \frac{1-\cos \left(\left(\Delta \epsilon_{k_{1}^{\prime}, k_{1}, k_{2}^{\prime}, k_{2}}\right) t\right)}{\left(\Delta \epsilon_{k_{1}^{\prime}, k_{1}, k_{2}^{\prime}, k_{2}}\right)^{2}} \\
& \times\left(\left(1-n_{k_{1}^{\prime}}\right) n_{k_{2}}\left(1-n_{k_{2}^{\prime}}\right)+n_{k_{1}^{\prime}}\left(1-n_{k_{2}}\right) n_{k_{2}^{\prime}}\right): c_{k_{1} \uparrow}^{\dagger} c_{k_{1} \uparrow}: \delta_{k_{1}^{\prime}+k_{2}^{\prime}, k_{1}+k_{2}} \\
& +\frac{2 U^{2}}{\Omega^{2}} \sum_{k_{1}^{\prime}, k_{1}, k_{2}^{\prime}, k_{2}} \frac{1-\cos \left(\left(\Delta \epsilon_{k_{1}^{\prime}, k_{1}, k_{2}^{\prime}, k_{2}}\right) t\right)}{\left(\Delta \epsilon_{k_{1}^{\prime}, k_{1}, k_{2}^{\prime}, k_{2}}\right)^{2}}\left(1-n_{k_{2}^{\prime}}\right) n_{k_{2}}: c_{k_{1} \uparrow}^{\dagger} c_{k_{1} \uparrow}: \delta_{k_{1}^{\prime}+k_{2}^{\prime}, k_{1}+k_{2}} \\
& +\frac{2 U^{2}}{\Omega^{2}} \sum_{k_{1}^{\prime}, k_{1}, k_{2}^{\prime}, k_{2}} \frac{1-\cos \left(\left(\Delta \epsilon_{k_{1}^{\prime}, k_{1}, k_{2}^{\prime}, k_{2}}\right) t\right)}{\left(\Delta \epsilon_{k_{1}^{\prime}, k_{1}, k_{2}^{\prime}, k_{2}}\right)^{2}} \\
& \times n_{k_{1}^{\prime}}\left(n_{k_{2}^{\prime}}\left(1-n_{k_{2}}\right)-\left(1-n_{k_{2}^{\prime}}\right) n_{k_{2}}\right): c_{k_{1} \uparrow}^{\dagger} c_{k_{1} \uparrow}: \delta_{k_{1}^{\prime}+k_{2}^{\prime}, k_{1}+k_{2}} \\
& + \text { linearly independent terms } \\
& +O\left(U^{3}\right) \text {. }
\end{aligned}
$$

We convince ourselves that after interchanging indices most of the terms cancel out, yielding

$$
N_{\uparrow}(t)=\sum_{k} n_{k}+\sum_{k}: c_{k \uparrow}^{\dagger} c_{k \uparrow}:+ \text { linearly independent terms }+O\left(U^{3}\right) .
$$

As the black and red terms are time-independent, this part of the calculation is consistent with the conservation of the total spin-up particle number. In order to check that also the linearly independent terms vanish, we would need the second-order corrections for $F_{k, k_{1}, k_{2}^{\prime}, k_{2}}$, which are irrelevant for our calculation of the density-density correlation functions. 


\section{B.3 Variance of the total spin-up particle number}

As the total spin-up particle number $N_{\uparrow}$ is a conserved quantity, also its variance

$$
\left\langle N_{\uparrow}^{2}\right\rangle-\left\langle N_{\uparrow}\right\rangle^{2}
$$

should be time-independent. We can derive the variance from the equal-time connected density-density correlation function for parallel spins, $C_{x^{\prime}, x}^{\uparrow \uparrow}(t)$, by a summation over $x^{\prime}$ and $x$,

$$
\begin{aligned}
\sum_{x^{\prime}, x} C_{x^{\prime}, x}^{\uparrow \uparrow}(t) & =\sum_{x^{\prime}, x}\left\langle\boldsymbol{n}_{x^{\prime} \uparrow}(t) \boldsymbol{n}_{x \uparrow}(t)\right\rangle-\sum_{x^{\prime}, x}\left\langle\boldsymbol{n}_{x^{\prime} \uparrow}(t)\right\rangle\left\langle\boldsymbol{n}_{x \uparrow}(t)\right\rangle \\
& =\left\langle N_{\uparrow}^{2}\right\rangle-\left\langle N_{\uparrow}\right\rangle^{2} .
\end{aligned}
$$

Hence, the solution for $C_{x^{\prime}, x}^{\uparrow \uparrow}(t)$ from eq. 6.24 should be consistent with this. The summation over $x^{\prime}$ in eq. 6.24 yields a $\delta_{k^{\prime}, k}$ and we get

$$
\begin{aligned}
& \sum_{x^{\prime}, x} C_{x^{\prime}, x}^{\uparrow \uparrow}(t)=\sum_{k} n_{k}\left(1-n_{k}\right) \\
& -\frac{4 U^{2}}{\Omega^{2}} \sum_{k} n_{k}\left(1-n_{k}\right) \sum_{k_{1}, k_{2}^{\prime}, k_{2}} \frac{1-\cos \left(\left(\Delta \epsilon_{k, k_{1}, k_{2}^{\prime}, k_{2}}\right) t\right)}{\left(\Delta \epsilon_{k, k_{1}, k_{2}^{\prime}, k_{2}}\right)^{2}} \\
& \times\left(\left(1-n_{k_{1}}\right) n_{k_{2}^{\prime}}\left(1-n_{k_{2}}\right)+n_{k_{1}}\left(1-n_{k_{2}^{\prime}}\right) n_{k_{2}}\right) \delta_{k+k_{2}^{\prime}, k_{1}+k_{2}} \\
& +\frac{4 U^{2}}{\Omega^{2}} \sum_{k} n_{k}\left(1-n_{k}\right) \sum_{k_{1}, k_{2}^{\prime}, k_{2}} \frac{1-\cos \left(\left(\Delta \epsilon_{k, k_{1}, k_{2}^{\prime}, k_{2}}\right) t\right)}{\left(\Delta \epsilon_{k, k_{1}, k_{2}, k_{2}}\right)^{2}} n_{k_{2}^{\prime}}\left(1-n_{k_{2}}\right) \delta_{k+k_{2}^{\prime}, k_{1}+k_{2}} \\
& -\frac{4 U^{2}}{\Omega^{2}} \sum_{k} n_{k}\left(1-n_{k}\right) \sum_{k_{1}, k_{2}^{\prime}, k_{2}} \frac{1-\cos \left(\left(\Delta \epsilon_{k, k_{1}, k_{2}^{\prime}, k_{2}}\right) t\right)}{\left(\Delta \epsilon_{k, k_{1}, k_{2}^{\prime}, k_{2}}\right)^{2}} n_{k_{1}}\left(n_{k_{2}^{\prime}}-n_{k_{2}}\right) \delta_{k+k_{2}^{\prime}, k_{1}+k_{2}} \\
& -\frac{4 U^{2}}{\Omega^{2}} \sum_{k} n_{k} \sum_{k_{1}, k_{2}^{\prime}, k_{2}} \frac{1-\cos \left(\left(\Delta \epsilon_{k, k_{1}, k_{2}^{\prime}, k_{2}}\right) t\right)}{\left(\Delta \epsilon_{k, k_{1}, k_{2}^{\prime}, k_{2}}\right)^{2}}\left(1-n_{k_{1}}\right) n_{k_{2}^{\prime}}\left(1-n_{k_{2}}\right) \delta_{k+k_{2}^{\prime}, k_{1}+k_{2}} \\
& +\frac{4 U^{2}}{\Omega^{2}} \sum_{k} n_{k} \sum_{k_{1}, k_{2}^{\prime}, k_{2}} \frac{1-\cos \left(\left(\Delta \epsilon_{k_{k}, k_{1}, k_{2}^{\prime}, k_{2}}\right) t\right)}{\left(\Delta \epsilon_{k, k_{1}, k_{2}^{\prime}, k_{2}}\right)^{2}}\left(1-n_{k_{1}}\right) n_{k_{2}^{\prime}}\left(1-n_{k_{2}}\right) \delta_{k+k_{2}^{\prime}, k_{1}+k_{2}} \\
& +O\left(U^{3}\right) \text {. }
\end{aligned}
$$


The orange terms cancel out directly and the red terms can be rearranged such that

$$
\begin{aligned}
\sum_{x^{\prime}, x} C_{x^{\prime}, x}^{\uparrow \uparrow}(t)= & \sum_{k} n_{k}\left(1-n_{k}\right) \\
& -\frac{4 U^{2}}{\Omega^{2}} \sum_{k} n_{k}\left(1-n_{k}\right) \sum_{k_{1}, k_{2}^{\prime}, k_{2}} \frac{1-\cos \left(\left(\Delta \epsilon_{k, k_{1}, k_{2}^{\prime}, k_{2}}\right) t\right)}{\left(\Delta \epsilon_{k, k_{1}, k_{2}^{\prime}, k_{2}}\right)^{2}} \\
& \times\left(\left(1-n_{k_{1}}\right) n_{k_{2}^{\prime}}\left(1-n_{k_{2}}\right)+n_{k_{1}}\left(1-n_{k_{2}^{\prime}}\right) n_{k_{2}}\right) \delta_{k+k_{2}^{\prime}, k_{1}+k_{2}} \\
& +\frac{4 U^{2}}{\Omega^{2}} \sum_{k} n_{k}\left(1-n_{k}\right) \sum_{k_{1}, k_{2}^{\prime}, k_{2}} \frac{1-\cos \left(\left(\Delta \epsilon_{k, k_{1}, k_{2}^{\prime}, k_{2}}\right) t\right)}{\left(\Delta \epsilon_{k^{\prime}, k_{1}, k_{2}^{\prime}, k_{2}}\right)^{2}} \\
& +O\left(\left(1-n_{k_{1}}\right) n_{k_{2}^{\prime}}\left(1-n_{k_{2}}\right)+n_{k_{1}}\left(1-n_{k_{2}^{\prime}}\right) n_{k_{2}}\right) \delta_{k+k_{2}^{\prime}, k_{1}+k_{2}} \\
= & \sum_{k} n_{k}\left(1-n_{k}\right) \\
& +O\left(U^{3}\right) .
\end{aligned}
$$

This is clearly time-independent and hence consistent with the conservation of the variance of the total spin-up particle number. 


\section{Bibliography}

[1] Abeling, N. O., Cevolani, L., And Kehrein, S. Analysis of the buildup of spatiotemporal correlations and their bounds outside of the light cone. SciPost Physics 5 (2018), 52.

[2] Anderson, M. H., Ensher, J. R., Matthews, M. R., Wieman, C. E., and Cornell, E. A. Observation of Bose-Einstein condensation in a dilute atomic vapor. Science 269 (1995), 198.

[3] Anderson, P. W. Absence of diffusion in certain random lattices. Physical Review 109 (1958), 1492.

[4] Averitt, R. D., And Taylor, A. J. Ultrafast optical and far-infrared quasiparticle dynamics in correlated electron materials. Journal of Physics: Condensed Matter 14 (2002), R1357.

[5] Bakr, W. S., Peng, A., Tai, M. E., Ma, R., Simon, J., Gillen, J. I., Fölling, S., Pollet, L., AND GreINER, M. Probing the superfluid-to-Mott insulator transition at the singleatom level. Science 329 (2010), 547.

[6] Bauer, B., ANd Nayak, C. Area laws in a many-body localized state and its implications for topological order. Journal of Statistical Mechanics: Theory and Experiment 2013 (2013), P09005.

[7] Berges, J., Borsányi, S., And Wetterich, C. Prethermalization. Physical Review Letters 93 (2004), 142002.

[8] Bloch, I. Ultracold quantum gases in optical lattices. Nature Physics 1 (2005), 23.

[9] Bloch, I., Dalibard, J., And Zwerger, W. Many-body physics with ultracold gases. Reviews of Modern Physics 80 (2008), 885.

[10] Bonnes, L., Essler, F. H. L., ANd Läuchli, A. M. "Light-cone” dynamics after quantum quenches in spin chains. Physical Review Letters 113 (2014), 187203.

[11] Bovensiepen, U., AND Kirchmann, P. Elementary relaxation processes investigated by femtosecond photoelectron spectroscopy of two-dimensional materials. Laser $\mathcal{E}$ Photonics Reviews 6 (2012), 589.

[12] Bravyi, S., Hastings, M. B., and Verstraete, F. Lieb-Robinson bounds and the generation of correlations and topological quantum order. Physical Review Letters 97 (2006), 050401. 
[13] Breuer, H.-P., and Petruccione, F. The Theory of Open Quantum Systems. Oxford University Press, 2002.

[14] Bronshtein, I. N., Semendyayev, K. A., Musiol, G., and Muehlig, H. Handbook of Mathematics, 5th ed. Springer, 2007.

[15] Calabrese, P., and Cardy, J. Evolution of entanglement entropy in one-dimensional systems. Journal of Statistical Mechanics: Theory and Experiment 2005 (2005), P04010.

[16] Calabrese, P., and Cardy, J. Quantum quenches in extended systems. Journal of Statistical Mechanics: Theory and Experiment 2007 (2007), P06008.

[17] Calabrese, P., Essler, F. H. L., and Fagotti, M. Quantum quench in the transversefield Ising chain. Physical Review Letters 106 (2011), 227203.

[18] Carleo, G., Becca, F., Sanchez-Palencia, L., Sorella, S., and Fabrizio, M. Lightcone effect and supersonic correlations in one- and two-dimensional bosonic superfluids. Physical Review A 89 (2014), 031602.

[19] Carleo, G., And Troyer, M. Solving the quantum many-body problem with artificial neural networks. Science 355 (2017), 602.

[20] Cavalleri, A. Photo-induced superconductivity. Contemporary Physics 59 (2018), 31.

[21] Cavalleri, A., Rini, M., Chong, H. H. W., Fourmaux, S., Glover, T. E., Heimann, P. A., Kieffer, J. C., And Schoenlein, R. W. Band-selective measurements of electron dynamics in $\mathrm{VO}_{2}$ using femtosecond near-edge $\mathrm{x}$-ray absorption. Physical Review Letters 95 (2005), 067405.

[22] Cazalilla, M. A. Effect of suddenly turning on interactions in the Luttinger model. Physical Review Letters 97 (2006), 156403.

[23] Cevolani, L., Carleo, G., and Sanchez-Palencia, L. Protected quasilocality in quantum systems with long-range interactions. Physical Review A 92 (2015), 041603.

[24] Cheneau, M., Barmettler, P., Poletti, D., Endres, M., Schauss, P., Fukuhara, T., Gross, C., Bloch, I., Kollath, C., and Kuhr, S. Light-cone-like spreading of correlations in a quantum many-body system. Nature 481 (2012), 484.

[25] Chiara, G. D., Montangero, S., Calabrese, P., and Fazio, R. Entanglement entropy dynamics of Heisenberg chains. Journal of Statistical Mechanics: Theory and Experiment 2006 (2006), P03001.

[26] Chin, C., Grimm, R., Julienne, P., And Tiesinga, E. Feshbach resonances in ultracold gases. Reviews of Modern Physics 82 (2010), 1225.

[27] Cohen, G., Gull, E., Reichman, D. R., and Millis, A. J. Taming the dynamical sign problem in real-time evolution of quantum many-body problems. Physical Review Letters 115 (2015), 266802.

[28] Collura, M., Calabrese, P., And Essler, F. H. L. Quantum quench within the gapless phase of the spin $-\frac{1}{2}$ Heisenberg XXZ spin chain. Physical Review B 92 (2015), 125131. 
[29] Davis, K. B., Mewes, M.-O., Andrews, M. R., van Druten, N. J., Durfee, D. S., Kurn, D. M., AND Ketterle, W. Bose-Einstein condensation in a gas of sodium atoms. Physical Review Letters 75 (1995), 3969.

[30] Delaunay, C. Théorie du mouvement de la lune. Comptes rendus des séances de l'Académie des Sciences 64 (1867).

[31] Eckstein, M., and Kollar, M. Nonthermal steady states after an interaction quench in the Falicov-Kimball model. Physical Review Letters 100 (2008), 120404.

[32] Eckstein, M., Kollar, M., and Werner, P. Thermalization after an interaction quench in the Hubbard model. Physical Review Letters 103 (2009), 056403.

[33] Eckstein, M., Kollar, M., AND Werner, P. Interaction quench in the Hubbard model: Relaxation of the spectral function and the optical conductivity. Physical Review B 81 (2010), 115131.

[34] Einstein, A. Über einen die Erzeugung und Verwandlung des Lichtes betreffenden heuristischen Gesichtspunkt. Annalen der Physik 322 (1905), 132.

[35] Essler, F. H. L., Frahm, H., Göhmann, F., Klümper, A., and Korepin, V. E. The One-Dimensional Hubbard Model. Cambridge University Press, 2005.

[36] Essler, F. H. L., Kehrein, S., Manmana, S. R., And Robinson, N. J. Quench dynamics in a model with tuneable integrability breaking. Physical Review B 89 (2014), 165104.

[37] FANO, U. Effects of configuration interaction on intensities and phase shifts. Physical Review 124 (1961), 1866.

[38] Fausti, D., Tobey, R. I., Dean, N., Kaiser, S., Dienst, A., Hoffmann, M. C., Pyon, S., Takayama, T., Takagi, H., and Cavalleri, A. Light-induced superconductivity in a stripe-ordered cuprate. Science 331 (2011), 189.

[39] Fedoryuk, M. V. The stationary phase method and pseudodifferential operators. Russian Mathematical Surveys 26 (1971), 65.

[40] Feshbach, H. A unified theory of nuclear reactions. II. Annals of Physics 19 (1962), 287.

[41] Först, M., Tobey, R. I., Wall, S., Bromberger, H., Khanna, V., Cavalieri, A. L., Chuang, Y.-D., Lee, W. S., Moore, R., Schlotter, W. F., Turner, J. J., Krupin, O., Trigo, M., Zheng, H., Mitchell, J. F., Dhesi, S. S., Hill, J. P., and Cavalleri, A. Driving magnetic order in a manganite by ultrafast lattice excitation. Physical Review B 84 (2011), 241104.

[42] Georges, A., Kotliar, G., Krauth, W., and Rozenberg, M. J. Dynamical meanfield theory of strongly correlated fermion systems and the limit of infinite dimensions. Reviews of Modern Physics 68 (1996), 13. 
[43] Gerber, S., Yang, S.-L., Zhu, D., Soifer, H., Sobota, J. A., Rebec, S., Lee, J. J., Jia, T., Moritz, B., Jia, C., Gauthier, A., Li, Y., Leuenberger, D., Zhang, Y., Chaix, L., Li, W., Jang, H., Lee, J.-S., Yi, M., Dakovski, G. L., Song, S., Glownia, J. M., Nelson, S., Kim, K. W., Chuang, Y.-D., Hussain, Z., Moore, R. G., Devereaux, T. P., Lee, W.-S., Kirchmann, P. S., and Shen, Z.-X. Femtosecond electron-phonon lock-in by photoemission and x-ray free-electron laser. Science 357 (2017), 71.

[44] Gericke, T., Würtz, P., Reitz, D., Langen, T., and Ott, H. High-resolution scanning electron microscopy of an ultracold quantum gas. Nature Physics 4 (2008), 949.

[45] Giannetti, C., Capone, M., Fausti, D., Fabrizio, M., Parmigiani, F., and MiHAILOVIC, D. Ultrafast optical spectroscopy of strongly correlated materials and hightemperature superconductors: a non-equilibrium approach. Advances in Physics 65 (2016), 58.

[46] Glazek, S. D., and Wilson, K. G. Renormalization of Hamiltonians. Physical Review D 48 (1993), 5863.

[47] Goldstein, H., Poole, C. P., and Safko, J. L. Classical Mechanics, 3rd ed. Pearson, 2002.

[48] Greiner, M., Mandel, O., Esslinger, T., Hänsch, T. W., and Bloch, I. Quantum phase transition from a superfluid to a Mott insulator in a gas of ultracold atoms. Nature 415 (2002), 39.

[49] Greiner, M., Mandel, O., Hänsch, T. W., and Bloch, I. Collapse and revival of the matter wave field of a Bose-Einstein condensate. Nature 419 (2002), 51.

[50] Gring, M., Kuhnert, M., Langen, T., Kitagawa, T., Rauer, B., Schreitl, M., Mazets, I., Smith, D. A., Demler, E., And Schmiedmayer, J. Relaxation and prethermalization in an isolated quantum system. Science 337 (2012), 1318.

[51] Hackl, A., AND KeHrein, S. Real time evolution in quantum many-body systems with unitary perturbation theory. Physical Review B 78 (2008), 092303.

[52] Hacki, A., And Kehrein, S. A unitary perturbation theory approach to real-time evolution problems. Journal of Physics: Condensed Matter 21 (2009), 015601.

[53] Hastings, M. B. Locality in quantum systems. arXiv:1008.5137v1 [math-ph], 2010.

[54] Hertz, H. Ueber einen Einfluss des ultravioletten Lichtes auf die electrische Entladung. Annalen der Physik 267 (1887), 983.

[55] Hubbard, J. Electron correlations in narrow energy bands. Proceedings of the Royal Society of London A: Mathematical, Physical and Engineering Sciences 276 (1963), 238.

[56] Hubbard, J. Electron correlations in narrow energy bands III. An improved solution. Proceedings of the Royal Society of London A: Mathematical, Physical and Engineering Sciences 281 (1964), 401. 
[57] Ichikawa, H., Nozawa, S., Sato, T., Tomita, A., Ichiyanagi, K., Chollet, M., Guerin, L., Dean, N., Cavalleri, A., Adachi, S.-I., Arima, T.-H., Sawa, H., Ogimoto, Y., Nakamura, M., Tamaki, R., Miyano, K., and Koshihara, S.-Y. Transient photoinduced 'hidden' phase in a manganite. Nature Materials 10 (2011), 101.

[58] Jördens, R., Strohmaier, N., Günter, K., Moritz, H., and Esslinger, T. A Mott insulator of fermionic atoms in an optical lattice. Nature 455 (2008), 204.

[59] Kastner, M. Entanglement-enhanced spreading of correlations. New Journal of Physics 17 (2015), 123024.

[60] Kehrein, S. The Flow Equation Approach to Many-Particle Systems. Springer, 2006.

[61] Kinoshita, T., Wenger, T., And Weiss, D. S. A quantum Newton's cradle. Nature 440 (2006), 900.

[62] Kiryukhin, V., Casa, D., Hill, J. P., Keimer, B., Vigliante, A., Tomioka, Y., And ToKurA, Y. An x-ray-induced insulator-metal transition in a magnetoresistive manganite. Nature 386 (1997), 813.

[63] Kitagawa, T., Imambekov, A., Schmiedmayer, J., and Demler, E. The dynamics and prethermalization of one-dimensional quantum systems probed through the full distributions of quantum noise. New Journal of Physics 13 (2011), 073018.

[64] KöHl, M., Moritz, H., Stöferle, T., Günter, K., And Esslinger, T. Fermionic atoms in a three dimensional optical lattice: Observing Fermi surfaces, dynamics, and interactions. Physical Review Letters 94 (2005), 080403.

[65] Kollar, M., Wolf, F. A., and Eckstein, M. Generalized Gibbs ensemble prediction of prethermalization plateaus and their relation to nonthermal steady states in integrable systems. Physical Review B 84 (2011), 054304.

[66] Kondov, S. S., McGehee, W. R., Xu, W., and DeMarco, B. Disorder-induced localization in a strongly correlated atomic Hubbard gas. Physical Review Letters 114 (2015), 083002.

[67] Kreye, M., And Kehrein, S. Prethermalization of density-density correlations after an interaction quench in the Hubbard model. arXiv:1908.10685 (2019).

[68] Kuво, R. Statistical-mechanical theory of irreversible processes. I. General theory and simple applications to magnetic and conduction problems. Journal of the Physical Society of Japan 12 (1957), 570.

[69] Kuzmany, H. Solid-State Spectroscopy, 2nd ed. Springer, 2009.

[70] Langen, T., Gasenzer, T., and Schmiedmayer, J. Prethermalization and universal dynamics in near-integrable quantum systems. Journal of Statistical Mechanics: Theory and Experiment 2016 (2016), 064009.

[71] Langen, T., Geiger, R., Kuhnert, M., Rauer, B., and Schmiedmayer, J. Local emergence of thermal correlations in an isolated quantum many-body system. Nature Physics 9 (2013), 640. 
[72] Langen, T., Geiger, R., and Schmiedmayer, J. Ultracold atoms out of equilibrium. Annual Review of Condensed Matter Physics 6 (2015), 201.

[73] Lichtenstein, A. I., And Katsnelson, M. I. Ab initio calculations of quasiparticle band structure in correlated systems: LDA + + approach. Physical Review B 57 (1998), 6884.

[74] Lieb, E. H., And Robinson, D. W. The finite group velocity of quantum spin systems. Communications in Mathematical Physics 28 (1972), 251.

[75] LindelöF, E. Sur l'application de la méthode des approximations successives aux équations différentielles ordinaires du premier ordre. Comptes rendus hebdomadaires des séances de l'Académie des sciences 116 (1894), 454.

[76] LäUChli, A. M., AND Kollath, C. Spreading of correlations and entanglement after a quench in the one-dimensional Bose-Hubbard model. Journal of Statistical Mechanics: Theory and Experiment 2008 (2008), P05018.

[77] Mankowsky, R., von Hoegen, A., Först, M., and Cavalleri, A. Ultrafast reversal of the ferroelectric polarization. Physical Review Letters 118 (2017), 197601.

[78] Manmana, S. R., Wessel, S., Noack, R. M., and Muramatsu, A. Time evolution of correlations in strongly interacting fermions after a quantum quench. Physical Review B 79 (2009), 155104.

[79] Mathias, S., Eich, S., Urbancic, J., Michael, S., Carr, A. V., Emmerich, S., Stange, A., Popmintchev, T., Rohwer, T., Wiesenmayer, M., Ruffing, A., Jakobs, S., Hellmann, S., Matyba, P., Chen, C., Kipp, L., Bauer, M., Kapteyn, H. C., Schneider, H. C., Rossnagel, K., Murnane, M. M., and Aeschlimann, M. Self-amplified photoinduced gap quenching in a correlated electron material. Nature Communications 7 (2016), 12902.

[80] Medvedyeva, M., Hoffmann, A., And Kehrein, S. Spatiotemporal buildup of the Kondo screening cloud. Physical Review B 88 (2013), 094306.

[81] Metzner, W., and Vollhardt, D. Correlated lattice fermions in $d=\infty$ dimensions. Physical Review Letters 62 (1989), 324.

[82] Miyano, K., Tanaka, T., Tomioka, Y., and Tokura, Y. Photoinduced insulator-tometal transition in a perovskite manganite. Physical Review Letters 78 (1997), 4257.

[83] Modugno, G., Ferlaino, F., Heidemann, R., Roati, G., and Inguscio, M. Production of a Fermi gas of atoms in an optical lattice. Physical Review A 68 (2003), 011601.

[84] Moeckel, M., And Kehrein, S. Interaction quench in the Hubbard model. Physical Review Letters 100 (2008), 175702.

[85] Moeckel, M., And Kehrein, S. Real-time evolution for weak interaction quenches in quantum systems. Annals of Physics 324 (2009), 2146. 
[86] Moeckel, M., AND Kehrein, S. Crossover from adiabatic to sudden interaction quenches in the Hubbard model: prethermalization and non-equilibrium dynamics. New Journal of Physics 12 (2010), 055016.

[87] Мотт, N. F. The basis of the electron theory of metals, with special reference to the transition metals. Proceedings of the Physical Society. Section A 62 (1949), 416.

[88] Motт, N. F. Metal-insulator transition. Reviews of Modern Physics 40 (1968), 677.

[89] Müller-Hartmann, E. Correlated fermions on a lattice in high dimensions. Zeitschrift für Physik B 74 (1989), 507.

[90] Nachtergaele, B., Raz, H., Schlein, B., and Sims, R. Lieb-Robinson bounds for harmonic and anharmonic lattice systems. Communications in Mathematical Physics 286 (2009), 1073.

[91] Nova, T. F., Cartella, A., Cantaluppi, A., Först, M., Bossini, D., Mikhaylovskiy, R. V., Kimel, A. V., Merlin, R., and Cavalleri, A. An effective magnetic field from optically driven phonons. Nature Physics 13 (2016), 132.

[92] Parsons, M. F., Mazurenko, A., Chiu, C. S., Ji, G., Greif, D., and Greiner, M. Siteresolved measurement of the spin-correlation function in the Fermi-Hubbard model. Science 353 (2016), 1253.

[93] Perfetti, L., Loukakos, P. A., Lisowski, M., Bovensiepen, U., Berger, H., Biermann, S., Cornaglia, P. S., Georges, A., and Wolf, M. Time evolution of the electronic structure of $1 T-\mathrm{TaS}_{2}$ through the insulator-metal transition. Physical Review Letters 97 (2006), 067402.

[94] Petsas, K. I., Coates, A. B., And Grynberg, G. Crystallography of optical lattices. Physical Review A 50 (1994), 5173.

[95] Piñeiro Orioli, A., AND Berges, J. Breaking the fluctuation-dissipation relation by universal transport processes. Physical Review Letters 122 (2019), 150401.

[96] Podolsky, D., Felder, G. N., Kofman, L., and Peloso, M. Equation of state and beginning of thermalization after preheating. Physical Review D 73 (2006), 023501.

[97] Polkovnikov, A., Sengupta, K., Silva, A., and Vengalattore, M. Colloquium: Nonequilibrium dynamics of closed interacting quantum systems. Reviews of Modern Physics 83 (2011), 863.

[98] Rammer, J., AND Smith, H. Quantum field-theoretical methods in transport theory of metals. Reviews of Modern Physics 58 (1986), 323.

[99] Reimann, P., And Dabelow, L. Typicality of prethermalization. Physical Review Letters 122 (2019), 080603.

[100] Rigol, M., Dunjko, V., Yurovsky, V., And Olshanis, M. Relaxation in a completely integrable many-body quantum system: An ab initio study of the dynamics of the highly excited states of $1 \mathrm{~d}$ lattice hard-core bosons. Physical Review Letters 98 (2007), 050405. 
[101] Rini, M., Tobey, R., Dean, N., Itatani, J., Tomioka, Y., Tokura, Y., Schoenlein, R. W., AND Cavalleri, A. Control of the electronic phase of a manganite by modeselective vibrational excitation. Nature 449 (2007), 72.

[102] Rozenberg, M. J., Zhang, X. Y., and Kotliar, G. Mott-Hubbard transition in infinite dimensions. Physical Review Letters 69 (1992), 1236.

[103] Schrader, D., Dotsenko, I., Khudaverdyan, M., Miroshnychenko, Y., RauschenBeutel, A., ANd Meschede, D. Neutral atom quantum register. Physical Review Letters 93 (2004), 150501.

[104] Schumm, T., Hofferberth, S., Andersson, L. M., Wildermuth, S., Groth, S., BarJoseph, I., SchmiedmaYer, J., AND KRÜGER, P. Matter-wave interferometry in a double well on an atom chip. Nature Physics 1 (2005), 57.

[105] SEIFERT, U. Stochastic thermodynamics, fluctuation theorems and molecular machines. Reports on Progress in Physics 75 (2012), 126001.

[106] Serbyn, M., Papić, Z., and Abanin, D. A. Local conservation laws and the structure of the many-body localized states. Physical Review Letters 111 (2013), 127201.

[107] Sherson, J. F., Weitenberg, C., Endres, M., Cheneau, M., Bloch, I., and Kuhr, S. Single-atom-resolved fluorescence imaging of an atomic Mott insulator. Nature 467 (2010), 68.

[108] Smith, D. A., Gring, M., Langen, T., Kuhnert, M., Rauer, B., Geiger, R., Kitagawa, T., Mazets, I., Demler, E., and Schmiedmayer, J. Prethermalization revealed by the relaxation dynamics of full distribution functions. New Journal of Physics 15 (2013), 075011.

[109] Stojchevska, L., Vaskivskyi, I., Mertelj, T., Kusar, P., Svetin, D., Brazovskit, S., AND Mihailovic, D. Ultrafast switching to a stable hidden quantum state in an electronic crystal. Science 344 (2014), 177.

[110] Tamasaku, K., Nakamura, Y., and Uchida, S. Charge dynamics across the $\mathrm{CuO}_{2}$ planes in $\mathrm{La}_{2-x} \mathrm{Sr}_{x} \mathrm{CuO}_{4}$. Physical Review Letters 69 (1992), 1455.

[111] Vasseur, R., And Moore, J. E. Nonequilibrium quantum dynamics and transport: from integrability to many-body localization. Journal of Statistical Mechanics: Theory and Experiment 2016 (2016), 064010.

[112] Vollhardt, D. Investigation of correlated electron systems using the limit of high dimensions. In Jerusalem Winter School for Theoretical Physics: Correlated Electron Systems, vol. 9. World Scientific, 1993.

[113] Vollhardt, D. Dynamical mean-field theory for correlated electrons. Annalen der Physik 524 (2012), 1.

[114] Wegner, F. Flow-equations for Hamiltonians. Annalen der Physik 506 (1994), 77. 
[115] White, S. R., And Feiguin, A. E. Real-time evolution using the density matrix renormalization group. Physical Review Letters 93 (2004), 076401.

[116] Wick, G. C. The evaluation of the collision matrix. Physical Review 80 (1950), 268. 


\section{Curriculum vitae}

\section{Manuel Kreye}

Nationality: German

PhD student: University of Göttingen

Thesis: A Unitary Perturbation Theory Approach to Real-Time Evolution in the Hubbard Model

Supervisor: Prof. Dr. Stefan Kehrein

Date of oral examination: 23rd October 2019

\section{Academic education}

2015 M.Sc. in Physics University of Göttingen

Thesis: Unrub Effect in Nonequilibrium Quench Dynamics

Supervisor: Prof. Dr. Stefan Kehrein

2013 B.Sc. in Physics University of Göttingen

Thesis: Electrons in an Open System Y-Geometry

Supervisor: Prof. Dr. Stefan Kehrein

\section{Publications \& Preprints}

(1) Kreye, M. and Kehrein, S. Prethermalization of density-density correlations after an interaction quench in the Hubbard model. arXiv:1908.10685 (2019). 